\title{
How Incentive Contracts and Task Complexity Influence and Facilitate Long-Term Performance
}

by

\section{Leslie Anne Berger}

\author{
A thesis \\ presented to the University of Waterloo \\ in fulfillment of the \\ thesis requirement for the degree of \\ Doctor of Philosophy \\ in \\ Accounting
}

Waterloo, Ontario, Canada, 2009

(C) Leslie Anne Berger 2009 
I hereby declare that I am the sole author of this thesis. This is a true copy of the thesis, including any required final revisions, as accepted by my examiners.

I understand that my thesis may be made electronically available to the public. 


\begin{abstract}
The purpose of this study is to investigate how different incentive contracts that include forward-looking and contemporaneous goals motivate managers to make decisions consistent with the organization's long-term objectives, in tasks of varying complexity. Two research questions are addressed. First, in a long-term horizon setting, how do incentive contracts based on various combinations of forward-looking and contemporaneous measures influence decisions? Second, how does task complexity influence the expected effect of various incentive contracts on management decisions?

I address my research questions using a multi-period experiment where I compare the effects of three different incentive structure types and two different levels of task complexity. Results show that in a low complexity task, individuals perform better when only contemporaneous goal attainment is rewarded in the incentive contract than when both forward-looking and contemporaneous goal attainment is rewarded. In a high complexity task, individuals perform better when both contemporaneous and forward-looking goal attainment is rewarded, but only when the contemporaneous goal attainment is weighted more heavily in the incentive contract.

My research contributes to the existing literature in two ways. First, this is the first study of which I am aware that compares the performance effects of long-term incentive contracts that reward forward-looking and contemporaneous goal attainment. Second, this study is the first of which I am aware to experimentally test incentive contracts, for employees with a long-term horizon, that incorporate various weightings of forward-looking measures in the contract. In addition, this study will be amongst the first to examine the impact of task complexity on incentive contract effectiveness.
\end{abstract}




\section{ACKNOWLEDGEMENTS}

I would like to gratefully acknowledge the extensive contributions made by my dissertation chair, Alan Webb. His numerous, and thoughtful, suggestions and constant encouragement have greatly benefited this thesis. I would also like to thank the rest of my dissertation committee, Alan Douglas, Derek Koehler, Sean Peffer and Tom Vance, for their helpful comments and suggestions. An earlier version of this thesis has also benefited from comments provided by Tony Atkinson, Efrim Boritz, Natalia Kotchetova, Theresa Libby, Guoping Liu, Patricia O’Brien, and workshop participants at Wilfrid Laurier University and Brock University. I am also grateful to Nancy Vadnen Bosch, Kareen Brown, and Sati Bandyopadhyay for their assistance in participant recruitment and to Jason Chan for programming the simulation used in this study. The financial assistance provided by the University of Waterloo and the Institute of Chartered Accountants of Ontario is gratefully acknowledged.

Finally, I am indebted to my family, Emmie, Rob, Katy, Linda and David. Without their endless patience, unconditional support, and encouragement this thesis would not have been possible. I would like to thank my parents for always encouraging me to challenge myself, and their incessant belief in my ability to succeed. I am grateful to Rob for his sense of humour and camaraderie throughout our $\mathrm{PhD}$ experience. Special thanks to Emmie for being an unwavering source of strength, encouragement, and optimism throughout this journey. 
This thesis is dedicated to my family.

To your health and happiness. 


\section{TABLE OF CONTENTS}

LIST OF FIGURES viii

CHAPTER 1: INTRODUCTION

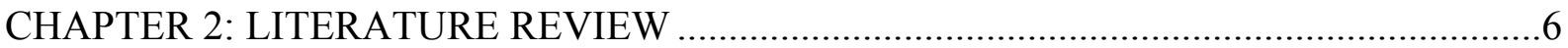

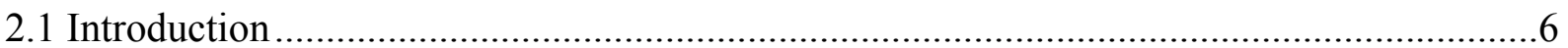

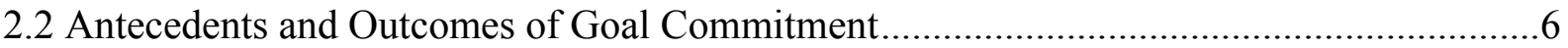

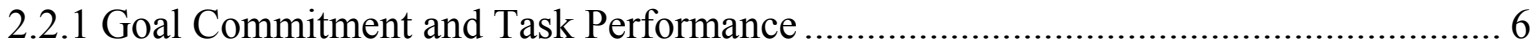

2.2.2 Goal Attractiveness and Self-Efficacy ……………............................................ 8

2.3 Forward-Looking and Contemporaneous Goals .............................................................10

2.4 Task Complexity as a Moderator of the Goal-Performance Relationship ...........................13

2.4.1 Simple and Complex Tasks.................................................................................. 13

2.4.2 Task Complexity and Goal Setting ……………………................................... 15

2.4.3 Forward-Looking and Contemporaneous Goals in Simple Tasks.............................. 16

2.4.4 Forward-Looking and Contemporaneous Goals in Complex Tasks ......................... 17

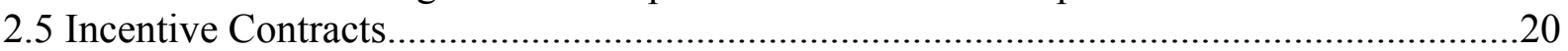

2.5.1 Performance Based Incentive Contracts.............................................................. 21

2.5.2 Challenges of Providing Incentives for the Attainment of Forward-looking Goals .. 23

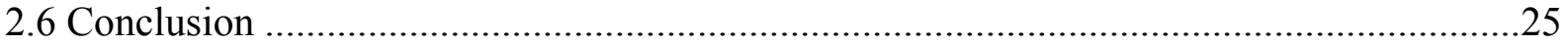

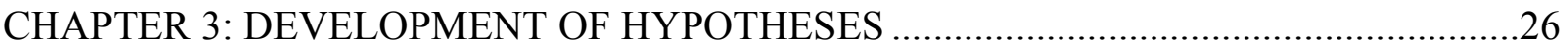

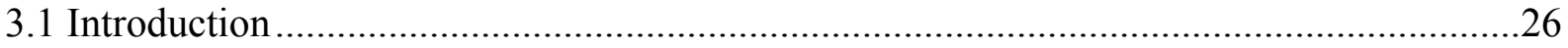

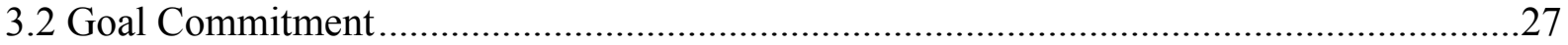

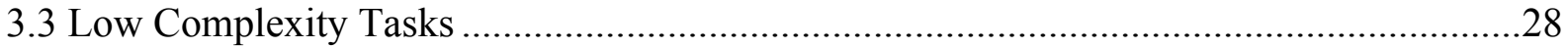

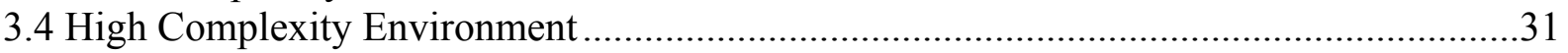

3.5 Limitations of Incentives on Forward-looking Goals ..........................................................34

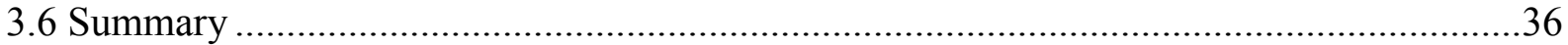

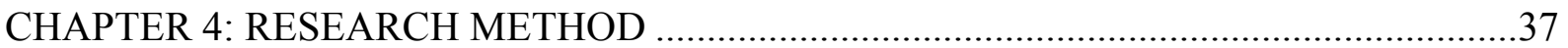

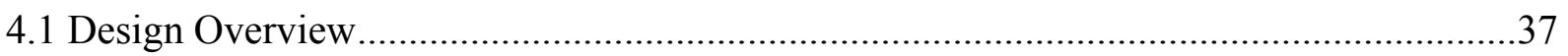

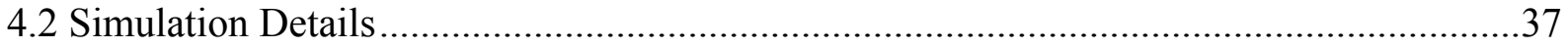

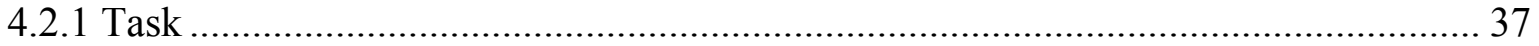

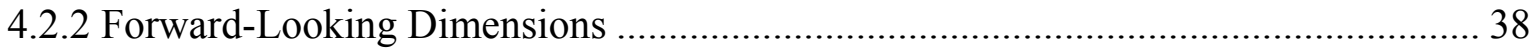

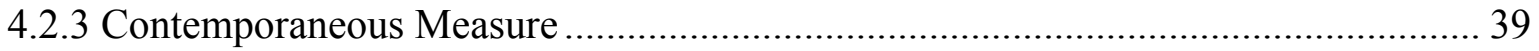

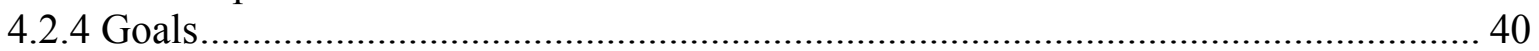

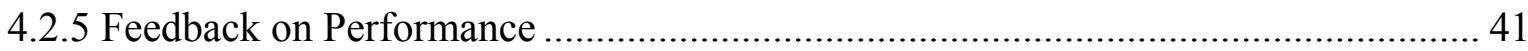

4.2.6 Work Horizon of the Employee ………………................................................. 42

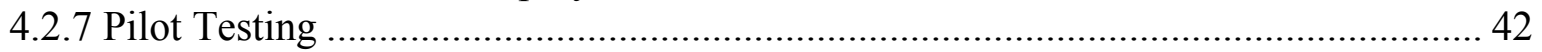

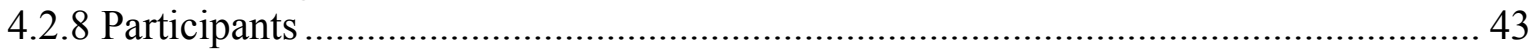

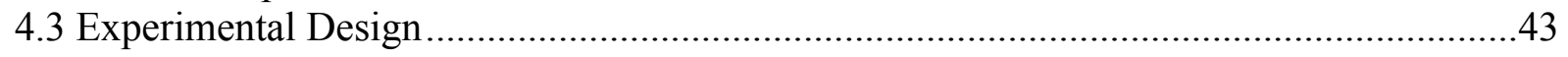

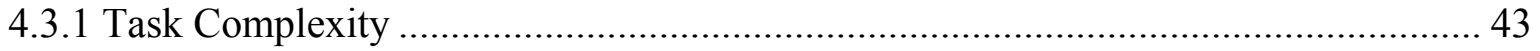

4.3.2 Performance Incentives ................................................................................... 44

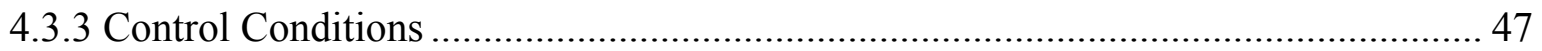




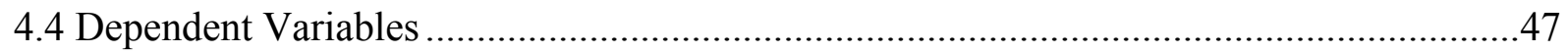

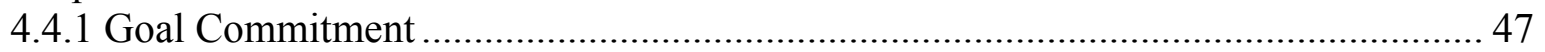

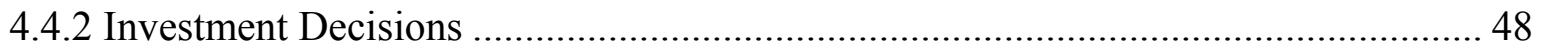

4.4.3 Complexity Manipulation Check ………………................................................. 48

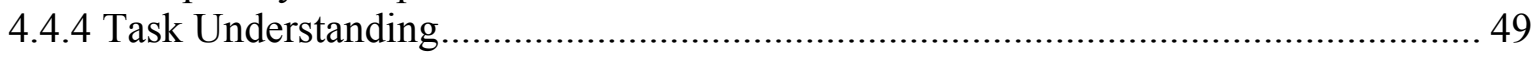

4.4.5 Controls and Other Measured Variables ……………......................................... 50

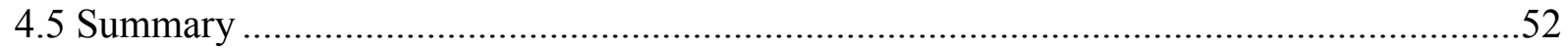

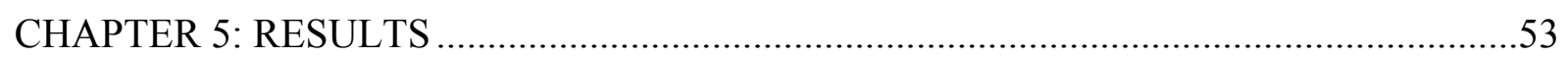

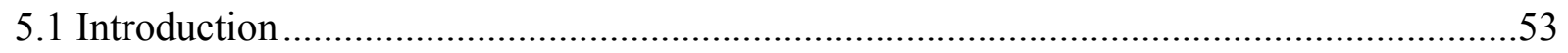

5.2 Demographic Information about Experimental Participants................................................53

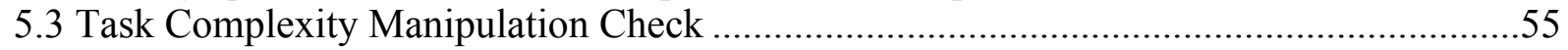

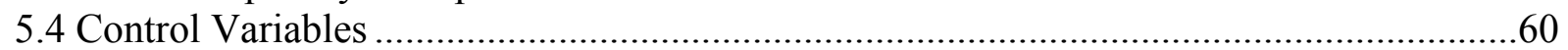

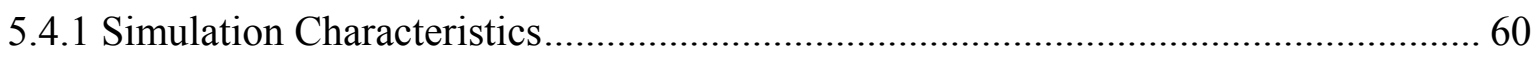

5.4.2 Perceptions About Importance of Forward-Looking Measures .................................. 61

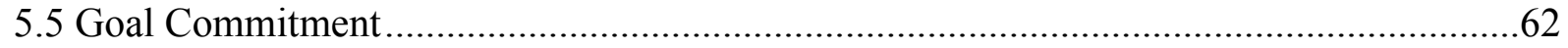

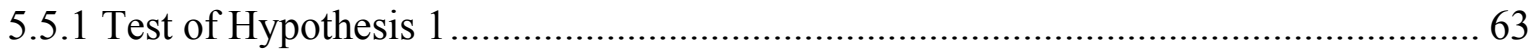

5.6 Investments in a Low Complexity Task (Test of Hypotheses 2 and 3) .............................67

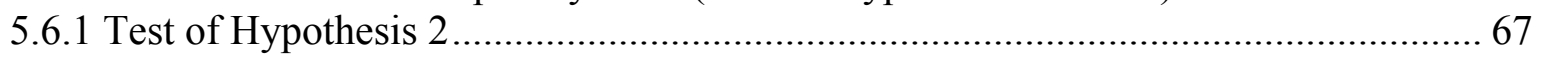

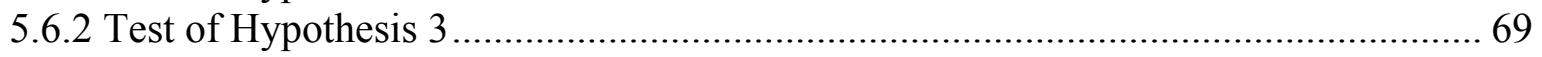

5.7 Investments in a High Complexity Task (Test of Hypotheses 4 and 6).............................71

5.8 Mediation in a High Complexity Task (Tests of Hypotheses 5 and 7) ………...................74

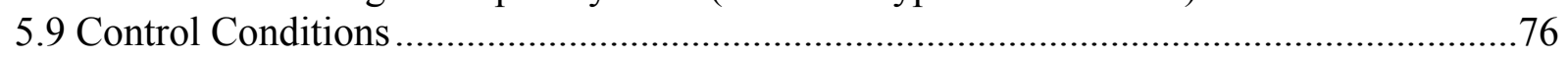

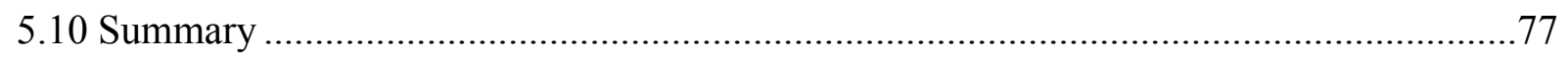

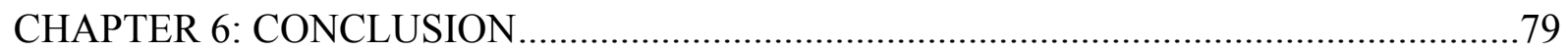

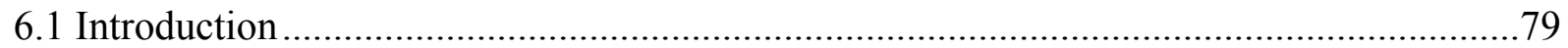

6.2 Discussion of Hypotheses Testing Results ...................................................................

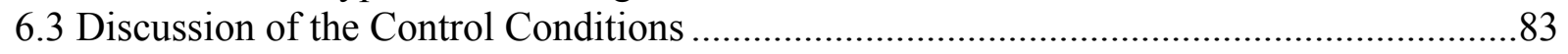

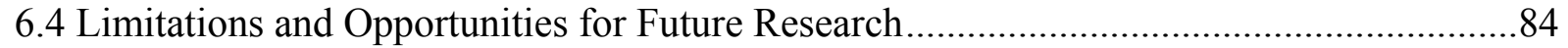

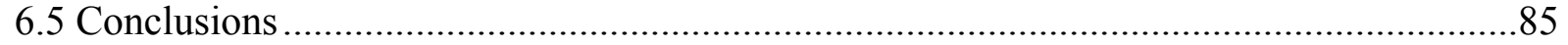

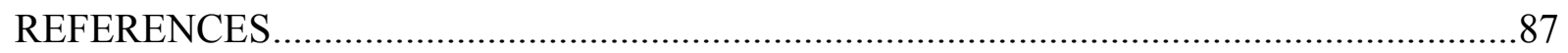

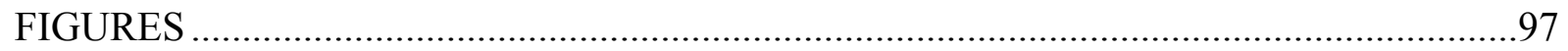

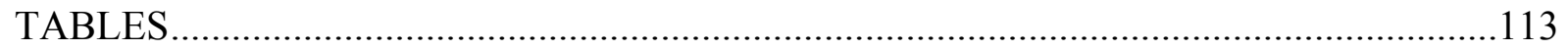

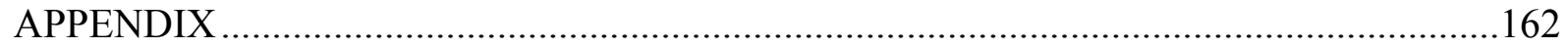

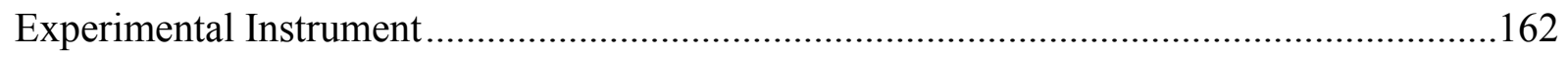




\section{LIST OF FIGURES}

1. Relationship between Forward-Looking and Contemporaneous Goals...........................97

2. Theoretical Relationships Introduced in Chapter 2 ......................................................98

3. Experimental Design .............................................................................................99

4. Hypothesized Relation between Incentive Contract Weights and Investment

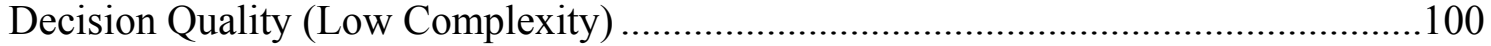

5. Hypothesized Mediation Relationship between Incentive Contract Weights and

Investment Decision Quality (Low Complexity) ......................................................101

6. Hypothesized Relation between Incentive Contract Weights and Investment

Decision Quality (High Complexity)

7. Hypothesized Mediation Relationship between Incentive Contract Weights and

Investment Decision Quality (High Complexity)....

8. Business Model Employed in the Experimental Simulation.

9. Relationship Between Food Quality Investments and Future Sales (High and

Low Complexity Conditions)

10. Cumulative Restaurant Net Income Periods 1 - 36 (Low Complexity)

11. Actual Relation between Incentive Contract Weights and Cumulative Net Income

(Low Complexity) .....

12. Actual Mediation Relationship between Incentive Contract Weights and

Investment Decision Quality (Low Complexity)

13. Cumulative Restaurant Net Income Periods 1 - 36 (High Complexity).

14. Actual Relation between Incentive Contract Weights and Cumulative Net Income

(High Complexity).

15. Actual Mediation Relationship between Incentive Contract Weights and

Investment Decision Quality (High Complexity) 


\section{LIST OF TABLES}

1. Relationships between Resource Allocation Decisions and Gross Income ......................113

2. Example Net Income Calculations........................................................................... 115

3. Incentives Paid for Attaining Assigned Goals .........................................................116

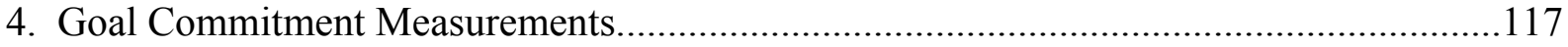

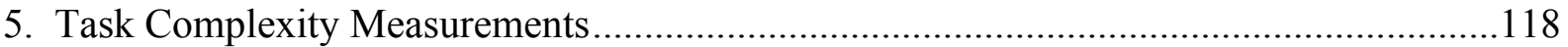

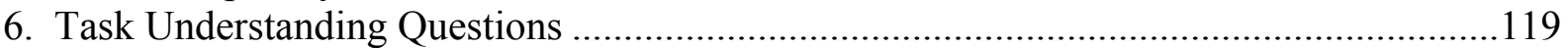

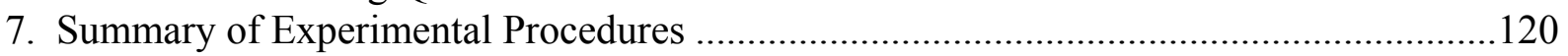

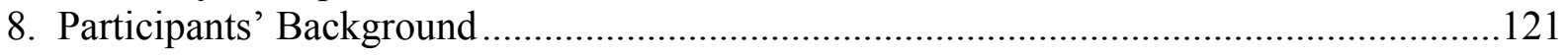

9. Task Complexity Manipulation Check - Questions asked at the end of Period 12

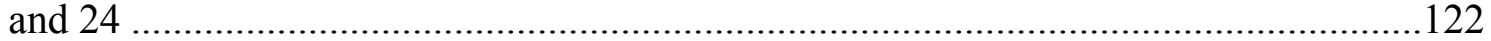

10. Task Complexity Manipulation Check - Questions asked at the end of Period 36 ..........125

11. Task Complexity Manipulation Check - Non Parametric Tests...................................129

12. Participants' Performance in Three Forward-Looking Measures Sets ...........................131

13. Comparison of Perceived Case Characteristics..........................................................132

14. Analysis of Participants' Perceptions of Forward-Looking Measures Importance ..........134

15. Reliability and Dimensionality of the Goal Commitment Scale.....................................137

16. Analysis of Goal Commitment Question Order........................................................139

17. Analysis of Goal Commitment Means by Incentive and Complexity Conditions............141

18. Analysis of Net Income - Low Complexity Condition .............................................145

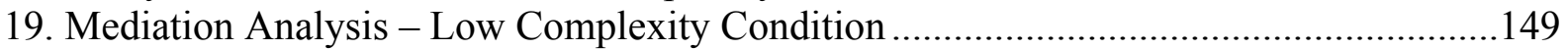

20. Analysis of Net Income - High Complexity Condition ................................................151

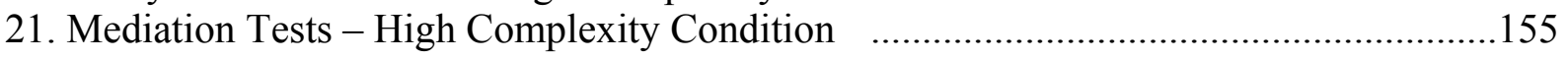

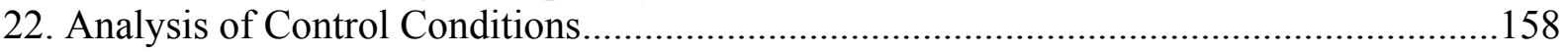

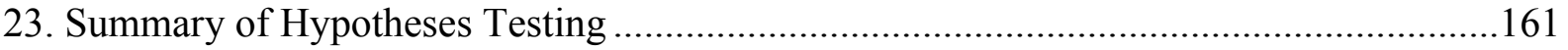




\section{CHAPTER 1: INTRODUCTION}

The purpose of this study is to investigate how different incentive contracts that include forward-looking (i.e., measures that predict future financial results) and contemporaneous (i.e., measures of current financial results) goals motivate managers to make decisions consistent with the organization's long-term objectives, in tasks of varying complexity. Two research questions are addressed. First, in a long-term horizon setting, how do incentive contracts based on various combinations of forward-looking and contemporaneous measures influence decisions? Second, how does task complexity influence the expected effect of various incentive contracts on management decisions?

An unresolved issue in the performance measurement literature is whether or not to include forward-looking measures in compensation contracts. There is evidence that many organizations do this (Ittner et al. 1997; Banker et al. 2000; Ittner et al. 2003; Bryant et al. 2004; Kaplan and Norton 2006). Further, in an experimental setting, Farrell et al. (2008) demonstrate that linking compensation to forward-looking measures may provide better longterm results. ${ }^{1}$ However, despite its potential decision facilitating benefits, forward-looking information can be difficult to effectively incorporate into incentive contracts. Indeed, some accounting researchers argue that the most effective contracts for employees with long-term commitments to the organization need not include forward-looking measures (Dikolli 2001; Sliwka 2002; Dutta and Reichelstein 2003). Incentive contracts that include forward-looking measures create opportunities for individuals to make decisions that maximize their own wealth, but are often to the detriment of long-term corporate results (Smith 2002; Meyer

\footnotetext{
${ }^{1}$ Although Farrell et al. (2008) conclude that forward-looking measures can provide information to employees with a long-term horizon, they do not consider the challenges of contracting on leading indicators in their study nor do they require participants to manage resources to achieve both forward-looking and contemporaneous measures.
} 
2002). ${ }^{2}$ For example, this problem can occur when the relationship between forward-looking performance and future contemporaneous results is non linear. In this setting, rewarding the performance of forward-looking measures often motivates individuals to excessively invest the organization's resources to maximize forward-looking measures, even when the incremental benefit of future financial results is negligible (Meyer 2002). This study contributes to the debate by comparing the performance effects of contracting only on contemporaneous measures to those of contracting on both forward-looking and contemporaneous measures.

Complex tasks can impede the performance effects of goals, and incentives rewarded for goal attainment (Wood et al. 2000). Therefore, an important consideration when examining the relationship between incentive contracts and employee performance is the complexity of the business environment. Tasks vary from highly complex, relatively difficult cognitive tasks, to less complex, relatively simple cognitive tasks (Goll and Rasheed 1997; Miller 1988). In less complex tasks, incentives rewarded for goal attainment have a direct, positive effect on performance through increased motivation, effort, and persistence (Libby 2001; Hannan 2005; Schulz et al. 2008). In more complex tasks, incentives rewarded for goal attainment do not have the same motivational effects on performance. Instead, assigned goals, and incentives rewarded for their attainment, can impair a manager's strategy development (Stajkovic and Luthans 1998; Wood et al. 1990). Indeed, some incentive contracts can divert attention from optimal strategies to suboptimal ones that fail to maximize manager and organizational performance (Smith 2002; Meyer 2002). This study examines

\footnotetext{
${ }^{2}$ Such misalignment of manager and organizational preferences can occur due to the following limitations of forward-looking information: it provides a noisy forecast of future returns that is less informative than awaiting actual results (Dutta and Reichelstein 2003); it is difficult to entirely identify and measure (Meyer 2002); and it is often not weighted accurately to reflect the company's business model (Krishnan et al. 2005).
} 
how different incentive contracts based on forward-looking and contemporaneous goal attainment influence performance, in tasks of low and high complexity.

I address my research questions using a multi-period experiment where participants, assigned a role of a restaurant manager, allocate resources between forward-looking measures (e.g., food quality and customer service). In this setting, participants' investments in forwardlooking measures reduce current period earnings but positively affect future contemporaneous results (i.e., net income). I compare the effects of three different incentive structure types and two different levels of task complexity. Consistent with my expectations, results show that in a low complexity task, individuals perform better (i.e. earn the organization more cumulative net income) when only contemporaneous goal attainment is rewarded in the incentive contract than when both forward-looking and contemporaneous goal attainment is rewarded. However, in a high complexity task, individuals perform better when both contemporaneous and forward-looking goal attainment is rewarded, but only when the contemporaneous goal attainment is weighted more heavily in the incentive contract.

My research contributes to the existing literature in two ways. First, this study extends accounting research that examines the use of forward-looking and contemporaneous measures in organizations. Existing research considers how organizations use forward-looking and contemporaneous measures to: manage their businesses (Malina and Selto 2001; Hendricks et al. 2004); establish relationships between forward-looking and contemporaneous results (Ittner and Larcker 1998; Bryant et al. 2004); and evaluate managers' performance (Lipe and Salterio 2000; Banker et al. 2004; Libby et al. 2004). Other research examines how individuals use forward-looking and contemporaneous information to make decisions (Webb 2004; Kelly 2007; Farrell et al. 2008). This is the first study of which I am aware that 
compares the performance effects of long-term incentive contracts that reward forwardlooking and contemporaneous goal attainment.

Second, this study contributes to the incentive contracting literature. Existing research examines the effect of incentives on employee horizon (Farrell et al. 2006), learning (Sprinkle 2000), employee effort (Hannan 2005), delegation (Nagar 2002) and performance (Fessler 2003). This study extends the literature by examining incentive effects in a long-term horizon setting. My research is the first to experimentally test incentive contracts, for employees with a long-term horizon that incorporate various weightings of forward-looking measures in the contract. In addition, this study will be amongst the first to examine the impact of task complexity on incentive contract effectiveness. In their recent review of managerial accounting research, Bonner and Sprinkle (2002) note that the effect of complexity on the relationship between incentives and performance is not fully understood and is worthy of future study.

This research also has practical significance to managers that design and implement performance measurement systems. In a recent survey by Deloitte (2007), senior management and board members clearly recognized the importance of forward-looking measures: $78 \%$ of those surveyed stated that contemporaneous measures alone did not adequately capture their company's results and 54\% acknowledged that forward-looking measures were of greater value to management than contemporaneous measures. However, the majority of those surveyed were not confident in their firms' ability to effectively monitor and communicate forward-looking results to decision makers (Deloitte 2007). This study provides practitioners with one method to communicate forward-looking results to decision makers. 
The remainder of the thesis is organized as follows. In Chapter 2, I use the psychology and accounting literature to examine how the assignment of goals, goal based incentives, and task complexity affect performance. In Chapter 3, I develop my predictions. In Chapter 4, I present the research design. In Chapter 5, I discuss the results of the hypotheses test, and in Chapter 6, I draw conclusions about the study's results. 


\subsection{Introduction}

\section{CHAPTER 2: LITERATURE REVIEW}

In this chapter, I use the existing psychology and accounting literature to examine how the assignment of goals, goal based incentives, and task complexity affect performance. This chapter is organized as follows. In Section 2.2, I examine the antecedents and outcomes of goal commitment. In Section 2.3, I introduce contemporaneous and forward-looking goals. In Section 2.4, I describe how task complexity moderates the goal-performance relationship. In Section 2.5, I examine how incentives rewarded for goal attainment can affect performance. This chapter concludes with Section 2.6.

\subsection{Antecedents and Outcomes of Goal Commitment}

In this section I describe how assigning a difficult, yet attainable, goal can have a positive effect on performance. I use theory found in the goal setting literature to show how high levels of goal attractiveness and self-efficacy positively affect goal commitment and attainment.

\subsubsection{Goal Commitment and Task Performance}

A well-established finding in the goal setting literature is that the assignment of a specific, challenging goal improves performance (Locke and Latham 2002). In a review of field and laboratory studies between 1969 to 1980, Locke et al. (1981) observe that, in 90\% of studies reviewed, the assignment of specific challenging goals lead to higher performance than the assignment of easy, do your best, or no goals. The assignment of a goal positively, and directly, impacts performance in three ways: by directing attention towards goal relevant activities, by motivating greater task relevant effort, and by increasing persistence with which 
goal relevant effort is exerted (Locke and Latham 2002). In addition, the presence of a goal can indirectly affect performance by leading to the discovery and use of task relevant knowledge and strategies (Locke and Latham 2002).

Goal commitment, defined as an individual's "determination to try, or keep trying, for a goal" (Locke et al. $1981 \mathrm{pg}$ 143) is a critical antecedent to goal attainment (Hollenbeck and Klein 1987; Locke et al. 1988; Hollenbeck et al. 1989; Klein et al. 2001; Locke and Latham 2002). Commitment to a goal is necessary to observe the positive effects of goals (Locke and Latham 2002) on performance. In a meta-analysis of 74 studies covering a 13-year period, Klein et al. (1999) report a positive relationship between goal commitment and performance and observe that goal commitment moderates the relationship between goal difficulty and performance. In addition to positive performance effects, the benefits of higher goal commitment include: an increase in participant performance aspirations and perseverance when faced with discrepant feedback (Earley and Lituchy 1991; Heath et al.1999; Latham and Seijts 1999) and an increased likelihood of developing effective strategies for accomplishing complex tasks (Wood and Bandura 1989; Latham et al. 1994).

Goal setting research demonstrates that the relationship between goal difficulty and high performance is stronger as the commitment to the goal increases (Klein et al. 1999). However, this observation is limited to settings where the difficult goal is still perceived by individuals as attainable (Locke et al. 1988). If a goal is perceived by individuals to be too difficult, or impossible, to attain they are less likely to commit to it (Klein 1991). For example, when participants, completing a puzzle-solving task, were assigned an impossible performance goal, they did not commit to the goal, and performance was negatively impacted 
(Earley et al. 1992). Indeed, in order to observe the positive relationship between difficult goals and performance, the goals assigned must be difficult, yet attainable.

\subsubsection{Goal Attractiveness and Self-Efficacy}

To establish high levels of goal commitment, high levels of goal attractiveness and self-efficacy are necessary (Locke et al. 1981; Hollenbeck and Klein 1987). In their metaanalysis, Klein et al. (1999) find positive effects of both efficacy and attractiveness on goal commitment. Goal attractiveness is defined as "the valence or desirability of (goal-related) performance" (Wright and Kacmar 1995, pg 265). Both personal (e.g., need for achievement, personality type, and work related attitudes) and situational factors (e.g., reward structures, competition, and publicity of personal performance) can affect goal attractiveness (Hollenbeck and Klein 1987). For example, in an experimental study, undergraduate students performing a word-descrambling task exhibited higher levels of goal attractiveness when offered monetary rewards for goal attainment (Klein and Wright 1994). In most circumstances, goals are more attractive when incentives are rewarded for their attainment because goal attainment allows participants to increase their own personal wealth through goal related performance (Hollenbeck and Klein 1987; Klein and Wright 1994; Locke and Latham 2002).

In a review of 131 experiments, Bonner et al. (2000) summarize the effects of incentives on performance. They observe that incentive contracts based on quota schemes (i.e., incentives paid for goal attainment) were more likely to positively affect performance than piece-rate, tournament or flat-rate incentive contracts. ${ }^{3}$ Bonner et al. (2000) attribute

\footnotetext{
${ }^{3}$ In a quota scheme, individuals receive a flat wage until a performance goal is attained. Once the goal is attained, workers receive a bonus and often a payment for each unit of output above the goal. In a piece rate
} 
these results to the benefits of increased goal attractiveness. The effect of incentives on performance has also been observed in a group setting. For example, Fisher et al. (2003) examine three different goal based incentive contracts: piece rate, budget fixed and budget linear. ${ }^{4}$ In comparing the three contracts, participants assigned the budget linear contract performed better than the participants assigned the other contracts.

Self-efficacy is a self-assessment of how well an individual can perform a task (Locke et al. 1984). Both personal factors (e.g. ability, self-esteem, and locus of control) and situational factors (e.g. task complexity, performance constraints and social influence) can affect the level of self-efficacy towards goal achievement (Hollenbeck and Klein 1987). For example, when assigned the task of identifying multiple uses for common objects, undergraduate business students with higher self-efficacy levels were more committed to their goals, and ultimately identified more uses for the objects than students with lower levels of self-efficacy (Locke et al. 1984). When individuals believe in their ability to attain a goal (i.e., high self-efficacy levels) they exhibit increased effort towards the goal, higher expectations for their own performance levels, and higher commitment to the goal; often resulting in increased performance levels (Locke et al. 1984).

In summary, assigning a difficult, yet attainable, goal has a positive effect on performance. The goal setting literature provides extensive evidence that high levels of goal attractiveness and self-efficacy positively affect goal commitment. Existing research also

scheme, individuals receive a predetermined amount for each unit of output. In a tournament scheme, individuals are paid based on an individual's performance ranking relative to other workers. The best performers earn the highest payment, while the worst performers earn the lowest payment. Flat-rate incentive contracts pay individuals a predetermined amount regardless of individual performance levels (Bonner et al. 2000, pgs 26-27).

${ }^{4}$ In a budget fixed contract, no bonus is earned when performance is below the goal, and a fixed bonus is earned once the goal has been achieved. In a budget linear contract, no bonus is earned when performance is below the goal, a bonus is earned for attainment of the goal, and a linear amount is earned for each unit of performance above goal. 
shows that high levels of goal commitment have a positive effect on performance and goal attainment.

\subsection{Forward-Looking and Contemporaneous Goals}

In this section, I discuss two types of goals, forward-looking and contemporaneous, examined in goal setting theory. Goal setting theory indicates that when forward-looking and contemporaneous goals are assigned, individuals must be committed to attaining both. However, if individuals become too committed to the forward-looking goals, relative to the contemporaneous goal, the existing theory shows that contemporaneous performance may be negatively impacted.

In recent years, different types of goals have been identified in the psychology and the accounting literature. One method to distinguish between goal types is to consider the temporal nature of the goals. A forward-looking goal (e.g., customer satisfaction) is short term in nature, designed to focus the decision maker on tasks that contribute to the achievement of a contemporaneous goal in a future period. A contemporaneous goal (e.g., net income) is a longer-term goal that focuses on the end result of a task. ${ }^{5}$ An example of this relationship is depicted in Figure 1.

Organizations often assign both contemporaneous and forward-looking goals to employees (Banker et al. 2000; Malina and Selto 2001; Ittner et al. 2003). One well-known example of a decision tool that provides both forward-looking and contemporaneous goals and actual results to employees is Kaplan and Norton's Balanced Scorecard (BSC) (1993,

\footnotetext{
${ }^{5}$ The literature also refers to forward-looking goals as leading, proximal, learning, short-term and sub goals. The literature also refers to contemporaneous goals as lagged, distal, performance, achievement, long-term and outcome goals. For the purposes of this study, and to be consistent with accounting research in this area (e.g., Farrell et al. 2008) I use the terms forward-looking and contemporaneous goals.
} 
1996). Widely adopted by organizations (Malina and Selto 2001; Bryant et al. 2004), the information provided by a Balanced Scorecard allows employees to monitor current period investments and identify strategies to maximize future performance (Kaplan and Norton 1996, 2006).

Forward-looking goals can serve two different roles within the organization. Forwardlooking goals may be decision facilitating because they can "provide the necessary information for managers to plan and make decisions" in an effort to attain the contemporaneous goal (Sprinkle 2003 pg 288). Forward-looking goals can also be decision influencing because they can "motivate individuals" (Sprinkle 2003 pg 288) to perform in a manner such that the organizational objectives (i.e., contemporaneous goal attainment) are attained (Demski and Feltham 1976).

Recent field and archival studies confirm the importance of forward-looking goals in facilitating management decisions by demonstrating a positive performance relationship between forward-looking and contemporaneous goals (Ittner et al. 1998; Banker et al. 2000; Bryant et al. 2004). This relationship is often non-linear, where after a certain amount of investment, additional investments have a diminishing benefit on future contemporaneous results (Ittner et al. 1998). For example, research shows that after a certain level of customer satisfaction is established, allocating additional resources to improving customer satisfaction will not continue to increase the likelihood of future customer retention, or future customer referrals (Ittner et al. 1998; Smith 2002).

The decision facilitating effects of forward-looking goals are only beneficial when individuals remain committed to contemporaneous goal attainment (Fishbach et al. 2006). If individuals' commitment to the contemporaneous goal is relatively low, and forward-looking 
goal commitment is relatively high, forward-looking goals can become decision influencing, instead of decision facilitating (Camerer et al. 1997; Fishbach et al. 2006). In other words, individuals become too focused on simply attaining the forward-looking goals, instead of thinking about them as a means of attaining the contemporaneous goals. Excessive focus on attaining forward-looking goals can lead to a less effective strategy selection that may not result in contemporaneous goal attainment (Fishbach et al. 2006). ${ }^{6}$ For example, in a experimental study of students trying to lose weight, Fishbach et al. (2006) show that students highly committed to a single, forward-looking goal (i.e., complete one 30-minute exercise session) selected strategies to attain it while dismissing opportunities to attain other forwardlooking (e.g., eating well) and contemporaneous goals (e.g., weight loss). This effect occurs because people develop strategies to attempt to attain the goal to which they are most committed (Wood et al. 1987; Chesney and Locke 1991; Camerer et al. 1997). A similar problem occurs in a business environment, where managers may select strategies to attain or exceed customer satisfaction goals (i.e., the forward-looking goal) while neglecting strategies that maximize organizational net income (i.e., the contemporaneous goal) (Smith 2002).

In a business environment, some commitment to a forward-looking goal is necessary to ensure that managers attend to information provided by the forward-looking goals that can help them attain the contemporaneous goal (Webb 2004). In an experimental study that examines manager behaviour in a multiple-goal setting, Webb (2004) observes that managers who exhibit high self-efficacy towards assigned forward-looking goals are more likely to be committed to both the forward-looking and contemporaneous goals.

\footnotetext{
${ }^{6}$ In this study, I examine a setting where high forward-looking measure performance (e.g., customer satisfaction) has a non-linear relationship with future contemporaneous results (e.g. net income). In this setting, exceeding forward-looking goals (i.e., with high levels of performance in the forward-looking measure) will not positively impact the decision maker's ability to attain contemporaneous goals.
} 
In summary, the existing literature suggests that when forward-looking and contemporaneous goals are assigned, individuals must be committed to attaining both. However, if individuals become too committed to the forward-looking goals, relative to the contemporaneous goal, contemporaneous performance may be negatively impacted.

\subsection{Task Complexity as a Moderator of the Goal-Performance Relationship}

In this section, I examine the moderating role of task complexity in the goalperformance relationship. Goal setting theory suggests that that in less complex (i.e., simple) tasks the assignment of, and commitment to, the contemporaneous goal may be sufficient for good contemporaneous performance. However, as task complexity increases, the assignment of only a contemporaneous goal could have negative performance effects. Goal setting theory shows that providing both forward-looking and contemporaneous goals can help decision makers to ultimately improve contemporaneous performance.

\subsubsection{Simple and Complex Tasks}

An important moderator of the positive relationship between goal assignment and performance is task complexity (Locke and Latham 2002). Task complexity is defined as the amount of processing or attention required by a task, or the amount of structure or clarity provided by a task (Bonner and Sprinkle 2002, pg 319). Business environments vary from settings where managing is a relatively complex task (e.g., management of an international, multi-location automotive company), to less complex settings (e.g., management of a single location coffee shop) where managing is low in complexity (Miller 1988; Goll and Rasheed 1997). The complexity of the task alters the effect of goals on individuals' performance 
(Wood et al. 2000), the effort participants direct to strategy development (Bonner and Sprinkle 2002), and the relationship between effort and performance (Bonner and Sprinkle 2002). Indeed, complexity is considered to be one of the most important determinants of performance in accounting settings (Bonner 1994; Bonner and Sprinkle 2002). Thus, in order to fully predict how goal assignment will affect a decision maker's performance, the complexity of the task must be considered.

Much of the incentive contracting and goal setting literature in accounting (Libby 2001; Hannan 2005; Schulz et al. 2008) and psychology (Heath et al. 1999; Locke and Latham 2002; Seijts and Latham 2005) examines participant performance using low complexity tasks. A low complexity task is one where performance is largely a function of the effort exerted. Low complexity tasks used in prior research include solving math problems (Earley and Lituchy 1991), translating symbols into letters of the alphabet (Libby 2001), and recoding three digit numbers into alphabet letters (Schulz et al. 2008). Such tasks allow researchers to examine the effects of goals and incentives on performance where relatively low amounts of cognitive processing or attention are required for task completion. In low complexity tasks, goals have a positive effect on performance through increased motivation and effort to meet the assigned goals (Wood et al. 1987; Locke and Latham 2002).

Higher complexity tasks require more information processing and attention by individuals than simple tasks. Complex tasks often include one of the following characteristics: multiple ways to arrive at the desired outcome, multiple possible outcomes, sequential completion of a series of related tasks, conflicting decisions, and outcome uncertainty (Campbell 1988; Bonner et al. 2000; Wood et al. 2000; Bonner and Sprinkle 2002). Complex tasks used in prior research include: the allocation of employees to different 
production subfunctions (Bandura and Wood 1989; Wood et al. 1990), making strategic management decisions using a business simulation game (Chesney and Locke 1991), creating stock portfolios for clients (Gilliland and Landis 1992) and considering financial statement audit procedures (Bonner et al. 2000).

\subsubsection{Task Complexity and Goal Setting}

In a meta-analysis of 125 studies, Wood et al. (1987) observe that goal-setting effects are strongest for simple tasks and weakest for complex tasks. In other words, the assignment of a goal for a complex task frequently does not result in the positive performance effects observed in simple tasks (Kanfer et al. 1994; Winters and Latham 1996). In an experimental study where participants were asked to perform a complex series of air traffic control tasks, those assigned a goal based on overall performance performed worse than those not assigned a goal but simply told to do their best (Kanfer et al. 1994). Wood et al. (1990) compared employee allocation decisions of managers completing a task of either low or high complexity. In their experiment, task complexity increased with the number of employees managers were required to allocate to various jobs. Wood et al. (1990) show that the assignment of a challenging goal positively impacted performance in the low complexity condition, but not in the high complexity condition.

The positive effects of goals on performance become weaker as task complexity increases because increased task related effort and task attention do not necessarily lead to performance improvements unless individuals exert sufficient cognitive effort to identify successful task strategies (Wood 1986; Wood et al. 1987; Campbell 1988; DeShon and Alexander 1996; Bonner and Sprinkle 2002). In complex tasks, assigned goals can positively 
influence the decision maker's strategy development (Stajkovic and Luthans 1998) by drawing their attention to information that assists in the development of successful task strategies (Wood et al. 1990). For example, in a complex class-scheduling task, assignment of a forward-looking goal (i.e., identify shortcuts useful in scheduling classes) drew participants attention to information that was useful in developing good task related strategies and would ultimately lead to the attainment of the contemporaneous goal (i.e., completed, and correct, class schedules). ${ }^{7}$ Thus, the level of task complexity must be considered when evaluating the goal-performance relationship.

\subsubsection{Forward-Looking and Contemporaneous Goals in Simple Tasks}

In a simple (i.e., less complex) task, the strategies required to attain a contemporaneous goal are often easily identified and implemented by individuals. When individuals are already aware of, or can quickly learn, successful strategies, the decision facilitating benefits provided by forward-looking goals are less consequential (Wood et al. 1990; Winters and Latham 1996). However in simple task settings, excessive commitment to forward-looking goals caused, for example, by incentives rewarded for their attainment can have a decision influencing effect that may negatively influence individuals' contemporaneous goal performance. ${ }^{8}$ Excessive commitment to forward-looking goals can cause individuals to select strategies that attain the forward-looking goal, while neglecting the consequences of their decisions on the attainment of the contemporaneous goal (Wood et al.

\footnotetext{
${ }^{7}$ Participants in this experiment were assigned the task of producing unique class schedule where a number of constraints in schedule preparation where described (e.g., a maximum number of classes per day, etc.). To improve their task scheduling performance participants could identify useful scheduling shortcuts or strategies (e.g., scheduling night classes, recording times chronologically, etc.).

${ }^{8}$ The negative decision influencing effects of forward-looking measures are discussed in more detail in Section 2.3.
} 
1990). For example, in a simple task, Winters and Latham (1996) observed that participants assigned only a contemporaneous goal performed better than those that were assigned both a contemporaneous and forward-looking goal. ${ }^{9}$ Indeed, in a simple task the decision facilitating benefits of forward-looking goals are often limited.

\subsubsection{Forward-Looking and Contemporaneous Goals in Complex Tasks}

One reason the goal performance relationship can be negatively impacted in complex tasks is if participants observe that their initial performance falls short of the level required by the goal and progress towards the goal is difficult to measure and evaluate (Heath et al. 1991). Referred to as the "starting problem" (Heath et al. 1999, pg 91), individuals often have low motivation to complete any goal congruent actions in complex tasks because they are overwhelmed by the perceived difficulty of the task, and doubt their ability to make effective decisions (Heath et al. 1999).

When individuals are assigned, and attend to, the attainment of both forward-looking and contemporaneous goals the starting problem can be reduced (Morgan 1985; Heath et al. 1999, Latham and Seijts 1999). For example, in a laboratory experiment where undergraduate students completed a scheduling task, Seijts and Latham (2001) observed that when participants were assigned forward-looking goals (identification of scheduling shortcuts) they were more likely to attain the contemporaneous goal (total number of schedules completed). When individuals believe that forward-looking and contemporaneous goals are causally related, the attainment of forward-looking goals can provide positive feedback to individuals about their current performance, which then increases the their self-

\footnotetext{
${ }^{9}$ In this experiment participants were assigned the task of producing unique class schedules. This task was a similar to the scheduling task used by Seijts and Latham (2001) described earlier in this section.
} 
efficacy for attaining the contemporaneous goal (Wood and Bandura 1989). Increased selfefficacy levels increase commitment to the contemporaneous goal, even if some forwardlooking goals in the future are not attained (Bandura and Simon 1977; Heath et al. 1999; Latham and Seijts 1999). To mitigate the starting problem in a complex environment it is important that employees be committed to attaining contemporaneous and forward-looking goals.

Individuals' self-efficacy and commitment towards the attainment of the contemporaneous goal is an important factor in motivational and learning processes that affect performance in complex tasks (Wood et al. 1990). For example, in a complex scheduling task, Winters and Latham (1996) show that participants not assigned forward-looking goals exhibit lower self-confidence in their ability to attain the contemporaneous goal, and are less likely to identify effective strategies, than participants' assigned forward-looking goals. When individuals are not confident in their ability to evaluate, process and integrate information to make good judgments, their performance is often negatively affected (Wood and Bandura 1989; Wood et al. 2000). In this situation, individuals often move unsystematically from one piece of information to another without thoroughly processing the information (Wood et al. 2000). Failure to process information causes individuals to ignore feedback that, when considered carefully, can provide insight into task performance (Diehl and Sterman 1995). In order to succeed in a complex task, individuals must develop a relatively accurate mental model, or understanding, of the relationships between feedback measures (i.e., forward-looking goals) and task outcomes (i.e., contemporaneous goals) 
(Atkins et al. 2002). ${ }^{10}$ Failure to do so often results in poor judgments based on superficial characteristics of the observed results (Wood et al. 2000). For example, in an inventory management task, Diehl and Sterman (1995) show that when participants neglected early feedback about inventory delays and gains, they were unable to develop an accurate mental model of the relationships in the inventory management system, and performed very poorly.

In complex tasks, people with high levels of self-efficacy and commitment towards the contemporaneous goal attempt to gather information for various alternatives and then process the information more systematically (Wood et al. 2000). Forward-looking goal performance provides cognitive feedback to individuals about the quality of their recent judgments in regards to the attainment of the contemporaneous goal (Kessler and Ashton 1981; Balzer et al. 1989). Cognitive feedback provides information about relationships between variables in a task. For example, in an experimental setting, when participants were assigned the task of repeatedly estimating a corporate bond rating based on the company's recent financial performance, the cognitive feedback they were provided included a summary of the accuracy of their previous assessments (Kessler and Aston 1981). Research shows that this type of feedback often improves the accuracy of judgments in many settings, including settings with multiple performance measures (Balzer et al. 1989).

Cognitive feedback can result in improved judgment quality in high complexity tasks (Balzer et al. 1989; Leung and Trotman 2005), and represents periodic information provided to the decision maker about their judgments (Kessler and Ashton 1981 pg 147). By thoroughly processing cognitive feedback individuals learn about the task at hand and as a result can develop a more accurate mental representation of the relationships between

\footnotetext{
${ }^{10}$ In my setting, I examine forward-looking measures that are causally linked to contemporaneous results. In this setting, performance on forward-looking measures, evaluated through the attainment of goals on those measures, can provide early feedback to individuals about future contemporaneous results.
} 
forward-looking and contemporaneous goals (Atkins et al. 2002; Leung and Trotman 2005). Individuals then use this knowledge to identify appropriate task-related strategies sooner, and more frequently, than individuals who do not possess an accurate mental representation of the task's causal model (Wood et al. 2000). All else being equal, successful strategy selection is expected to ultimately result in better performance because in complex tasks the quality of the strategy identified is typically related to the quality of task performance (Chesney and Locke 1991).

When a task is complex, the assignment of forward-looking and contemporaneous goals has been shown to increase individual's self-efficacy towards the attainment of contemporaneous goals, which in turn increases commitment to those goals (Heath et al. 1999). To attain the contemporaneous goal, individuals often use forward-looking goals to learn about effective strategies to employ when working towards the contemporaneous goal (Heath et al. 1999).

The research reviewed above shows that in simple task settings assignment of, and commitment to, the contemporaneous goal may be sufficient for good contemporaneous performance. However, as task complexity increases, the assignment of only a contemporaneous goal could have negative performance effects due to the starting problem. Research shows that providing both forward-looking and contemporaneous goals can help overcome the starting problem, and ultimately improve contemporaneous performance.

\subsection{Incentive Contracts}

In this section, I introduce theory, found in the incentive contracting literature, indicating that forward-looking goals should be included in incentive contracts to encourage 
employees to attend to them. I also introduce a competing theory, found in the agency and incentive contracting literature, that suggests that incentives rewarded for forward-looking goal attainment may encourage undesirable employee actions.

\subsubsection{Performance Based Incentive Contracts}

There is a considerable amount of evidence suggesting that performance based incentives can affect performance. Factors that can affect this relationship include the complexity of the task and, in cases were goal attainment is rewarded, the type of goal rewarded. In a review of 131 experimental studies, Bonner et al. (2000) observe that the positive relationship between financial incentives and performance is moderated by the complexity of the task. They observe that in simple tasks (e.g., product assembly) incentives have a positive, direct effect on performance but in complex tasks (e.g., solving word problems) incentives do not have an effect on performance. However, in most cases the studies examined short-term tasks where contemporaneous goal attainment, but not forwardlooking goal attainment, was rewarded. More recent experimental research has continued to examine the performance effects of contemporaneous goal based incentives in a complex task (Sprinkle 2000, Kelly 2007). For example, in a multi-period, complex task, participants assigned an incentive contract based on contemporaneous results (i.e., per-period profit) generate better performance than those paid only a flat wage (Sprinkle 2000).

An incentive contract commonly used in practice is one where multiple performance goals are incorporated into the bonus structure (Malina and Selto 2001; Ittner et al. 2003; Kaplan and Norton 2006). For example, organizations that use the BSC often reward employees for the attainment of forward-looking goals (e.g., customer satisfaction targets) in addition to contemporaneous goals (e.g. annual net income targets) (Kaplan and Norton 
2006). ${ }^{11}$ Moreover, in a recent survey by Deloitte (2007), senior management and board members clearly recognize the importance of forward-looking measures: $78 \%$ of those surveyed state that contemporaneous measures alone do not adequately capture their company's results and 54\% acknowledge that forward-looking measures are of greater value to management than contemporaneous measures. However, the majority of those surveyed are not confident in their firms' ability to effectively monitor and communicate forwardlooking results to individuals (Deloitte 2007). Thus, while practitioners understand the benefits of forward-looking measures they are not sure how to incorporate them into management decision-making or incentive contracts.

Existing accounting research has begun to examine the performance effects of incentive contracts that incorporate both forward-looking and contemporaneous goals. In some cases, simply assigning forward-looking goals without rewarding their attainment does not appear to motivate participants to sufficiently commit to them. The experimental accounting literature suggests that when incentives are tied only to contemporaneous goal achievement, the decision influencing properties of the forward-looking goals are limited (Kelly 2007; Farrell et al. 2008). Results suggest that individuals make better decisions when both forward-looking and contemporaneous goals are included in the incentive contract (Kelly 2007; Farrell et al. 2008; Kelly 2009). For example, in a complex, resource allocation task, Kelly (2007) demonstrates that participants generated higher levels of operating income (i.e., contemporaneous goal) when productivity and efficiency (i.e., forward-looking goals) were

\footnotetext{
${ }^{11}$ Organizations are often faced with the decision of how to allocate a fixed bonus amount between the various goals assigned to employees. Recent evidence suggests that a fixed bonus pool is common in industry (Bailey et al. 2006), which sets the maximum bonus amount to be earned by employees, regardless of how it is allocated across one or more performance measures. For example, a recent field study suggests that, although the method used to allocate bonuses varies, the amount of the bonus pool is fixed for about $60 \%$ of firms sampled (Murphy and Oyer 2003). Accordingly, if the total bonus pool is fixed, the more goals contracted upon the lower the incentive amount rewarded per individual goal attainment.
} 
also rewarded in the incentive contract. ${ }^{12}$ When incentives are provided only for contemporaneous goal attainment individuals' commitment can be diverted away from the forward-looking measures, even though these measures can provide insight on how to perform a task (Wood et al. 1990). To mitigate this problem, that attainment of both forwardlooking and contemporaneous goals should be rewarded.

2.5.2 Challenges of Providing Incentives for the Attainment of Forward-looking Goals

Contrary to the research discussed in section 2.5.1, some accounting researchers argue that, for many employees, the most effective contracts should not reward forward-looking goal attainment because of three issues (Dikolli 2001; Sliwka 2002; Dutta and Reichelstein 2003). First, forward-looking measures, although more timely, provide a noisy forecast of future returns that is less informative than awaiting actual results (Dutta and Reichelstein 2003). Second, it is difficult to identify and measure all possible forward-looking information that may provide inferences about future earnings (Meyer 2002). Third, weighting various forward-looking measures to accurately reflect the company's business model is a difficult task that is often not done well by mangers (Krishnan, Luft and Shields 2005).

When designing incentive contracts, the employment horizon of the employee must also be considered. A long-term horizon is defined in the agency literature as one where the agent's horizon nears that of the principal (Dikolli 2001). For example, compensation based on stock options or other vesting equity instruments encourages employees to view their decisions under a long-term horizon (Hall and Murphy 2003; Aseff and Santos 2005).

\footnotetext{
${ }^{12}$ Kelly $(2007,2009)$ examines decisions made by managers with a short-term employment horizon. Her research questions do not consider how a long-term employment horizon can affect the relationship between incentive contracts and management decisions. Kelly also does not consider how various, accurate weightings of forward-looking and contemporaneous goal based incentives in the incentive contract can affect long-term performance.
} 
Although not all employees within an organization have a long-term horizon, those with more responsibility may be more likely to view their work, and their lifespan at the company, from a longer-term perspective. Studying a long-term horizon setting is important because, unlike short-term horizon settings, employees with a long-term horizon have the opportunity to manage current resource allocations while observing (and potentially benefiting from) the long-term consequences of these decisions.

Research indicates that if an employee has a shorter time horizon than the organization, the use of a single, contemporaneous measure (e.g., net income) is not an appropriate incentive contract to reward performance. Instead, including rewards based on forward-looking measures (e.g., customer service) in the incentive contract directs the employee's short-term behaviour to be more congruent with the organization's long-term strategy (Feltham and Xie 1994; Dikolli 2001; Sliwka 2002; Dutta and Reichelstein 2003). ${ }^{13}$

There is little existing research that examines the performance of employees with a long-term horizon when the assigned incentive contracts are based on attaining both forwardlooking and contemporaneous goals. In an experiment where participants completed a simulated sandwich assembly task, Farrell et al. (2008) find that the inclusion of forwardlooking goals in an incentive contract influences the decisions of employees working with a short-term employment horizon, while facilitating the decisions of employees working with a long-term employment horizon. ${ }^{14}$ Although Farrell et al. (2008) conclude that forwardlooking measures can provide information to employees with a long-term horizon, they do not

\footnotetext{
${ }^{13}$ This assumes that performance on forward-looking measures is correlated with performance on contemporaneous measures.

${ }^{14}$ In this computerized task, participants filled a series of customer sandwich orders, where quantity and quality of production were considered.
} 
require participants to manage resources to achieve both forward-looking and contemporaneous goals.

The literature reviewed above suggests that forward-looking goals should be included in incentive contracts to encourage employees to attend to them. Other research indicates that incentives rewarded for forward-looking goal attainment may encourage undesirable employee actions, such as effort shifting (Meyer 2002; Smith 2002). Moreover, when employees have a long-term employment horizon, analytical research suggest that rewarding only the attainment of contemporaneous goals will result in more congruent employee behavior, than rewarding both forward-looking and contemporaneous goal attainment (Dikolli 2001).

\subsection{Conclusion}

This chapter reviewed relevant psychology and accounting research that examines the effect of goals on task performance. Figure 2 presents a summary of the theoretical relationships discussed in this chapter. Overall, the existing literature suggests that the assignment of forward-looking and contemporaneous goals results in better performance on complex tasks. However, results are equivocal regarding about the appropriate method to incorporate forward-looking goal attainment in incentive contracts. Some research suggests that rewarding forward-looking goal attainment is necessary, while other evidence indicates that rewarding forward-looking goal attainment can negatively impact contemporaneous performance results. This issue will be examined in more detail in Chapter 3 in developing the hypotheses. 


\section{CHAPTER 3: DEVELOPMENT OF HYPOTHESES}

\subsection{Introduction}

In this section I use goal setting theory to develop hypotheses about individuals' behaviour. I examine individual behaviour in a long-term environment when assigned both forward-looking and contemporaneous goals. My objective is to identify the incentive structures that motivate individuals to perform in a manner most consistent with the long-term objectives of the organization in tasks of high and low complexity.

I examine three different types of incentive contracts that reward contemporaneous goal attainment with a low, medium or high weighting in the total incentive contract (see

Figure 3). Contracts that assign a low or medium weighting to the contemporaneous goal also reward individuals for forward-looking goal attainment (e.g., Figure 3, Conditions A, B, D, and E). This represents a type of contract often used in industry where all performance measures are incorporated into the bonus structure (Kaplan and Norton 2006; Ittner et al. 2003; Malina and Selto 2001). The third contract has a high (100\%) weighting assigned to contemporaneous goal attainment and does not reward forward-looking goal attainment (Figure 3, Conditions $\mathrm{C}$ and F). This is the recommended incentive structure for long-term horizon individuals in the analytical literature (Sliwka 2002; Dikolli 2001; Dutta and Reichelstein 2003).

Several aspects of my research setting are important to note. First, I focus on organizations with the long-term objective to maximize earnings. Second, I examine individuals' decisions in allocating resources between various investments. Third, I define "better investment decisions" as ones that, when consistently implemented, meet the longterm performance objectives of the organization. Fourth, consistent with empirical evidence 
(Ittner et al. 1998) in my setting there is a non-linear relationship between performance on forward-looking measures and future contemporaneous performance. Finally, in my setting I assume that individuals are able to learn that forward-looking and contemporaneous goals are causally related.

This chapter is organized as follows. Section 3.2 develops my prediction regarding goal commitment. Sections 3.3, 3.4 and 3.5 develop two predictions regarding incentive structures and task complexity. Section 3.6 summarizes the chapter.

\subsection{Goal Commitment}

In order to predict the performance effects of the various compensation structures being studied, I first examine contemporaneous goal commitment levels. In this setting, the contemporaneous goal (i.e., net income) represents the organization's long-term objective to maximize earnings (Dikolli 2001; Kaplan and Norton 2006). In both high and low complexity tasks, commitment to the contemporaneous goal is essential to ensure that individuals' objectives are aligned with the organization's objective. Higher commitment to the contemporaneous goal, relative to the forward-looking goal, is desirable in most circumstances (Meyer 2002). ${ }^{15}$ Therefore, higher contemporaneous goal commitment is an important factor in achieving better performance.

As discussed in Chapter 2, situational factors, such as reward structures, can have a positive effect on goal commitment by increasing the attractiveness of goal attainment (Hollenbeck and Klein 1987; Locke et al. 1988). In the three incentive contracts under consideration, the higher the incentive weight assigned to contemporaneous goal attainment

\footnotetext{
${ }^{15}$ When employees only have a short-term horizon, commitment to (and ultimately the attainment) of forwardlooking goals is preferred to that of contemporaneous goals (Dikolli 2001; Meyer 2002).
} 
(relative to the reward for the attainment of the forward-looking goal) the more attracted individuals will be to the contemporaneous goal. From the individual's point of view, attainment of the contemporaneous goal in the high contemporaneous weighting condition (Conditions $\mathrm{C}$ and $\mathrm{F}$ ) will lead to a larger bonus, and thus will be more attractive than attaining the same goal in the low (Conditions A and D) or medium (Conditions B and E) contemporaneous weighting conditions. ${ }^{16,17}$ Because goal attractiveness is an antecedent of goal commitment (Locke et al. 1988), I expect that commitment to the contemporaneous goal will be highest when contracts only reward contemporaneous goal attainment. By the same reasoning, when incentives are provided for the attainment of both forward-looking and contemporaneous goals, the higher the incentive weight assigned to contemporaneous goal attainment, the higher the level of commitment to the contemporaneous goal. Therefore, I predict the following:

H1: As the weighting of incentives rewarded for contemporaneous goal attainment increases, individuals' commitment to the contemporaneous goal, relative to that of the forward-looking goal, will increase. (Figure 3, Condition $C$ and $F>B$ and $E>A$ and $D$ )

\subsection{Low Complexity Tasks}

As described in Chapter 2, a low complexity task requires a relatively low level of information processing in making management decisions (Dess and Beard 1984, $\mathrm{p} 56$ ). When individuals are given incentives for attainment of a goal on a low complexity task, they can

\footnotetext{
${ }^{16}$ In conditions $\mathrm{C}$ and $\mathrm{F}$ individuals are assigned contracts with high weight on the contemporaneous measure, where all of the performance based incentives result from the attainment of the contemporaneous goal. In conditions A, B, D and E individuals are assigned contracts where performance based incentives result from the attainment of the contemporaneous and forward-looking goals. Therefore, individuals earn less for the attainment of the contemporaneous goals in these conditions than the individuals in conditions $\mathrm{C}$ and $\mathrm{F}$. ${ }^{17}$ Because the level of task complexity is not expected to have an effect on goal commitment levels (Hollenbeck and Klein 198; Heath et al, 1999), I collapse the high and low complexity conditions in the development of this hypothesis.
} 
readily identify and make decisions that will lead to achievement of that goal (Dikolli 2001). Some of these decisions will result in performance that achieves the organization's objective, while others will not (Dikolli 2001; Sliwka 2002; Meyer 2002; Dutta and Reichelstein 2003). For example, a decision that leads to higher long-term earnings satisfies the organization's objective while a decision that increases customer satisfaction, but reduces long-term earnings, does not (Smith 2002). The organization can use goals, and the incentives rewarded for their attainment, to make desirable decisions (i.e., those that result in high long-term earnings) more attractive to individuals. In a low complexity task this can be accomplished by ensuring that commitment to the contemporaneous goals is relatively higher than that of forward-looking goals.

As discussed in Chapter 2, in low complexity tasks, individuals who are highly committed to the contemporaneous goal (i.e., net income) are likely to make decisions that result in contemporaneous goal attainment because the goal-performance relationship is strongest when high levels of goal commitment exist (Wood and Bandura 1989; Chesney and Locke 1991; Locke and Latham 2002). In my setting, attainment of the contemporaneous goal is desirable to the organization because this goal corresponds with the organization's objective of earnings maximization. However, if individuals are more committed to the forward-looking goals relative to the contemporaneous goals, they might make decisions that are designed to attain the forward-looking goals (Dikolli 2001; Meyer 2002). Such decisions could result in performance that may not be aligned with the organizational objective because forward-looking goal attainment does not always result in contemporaneous goal attainment (Dikolli 2001; Meyer 2002; Dutta and Reichelstein 2003). For example, initial investments in customer service positively affect the likelihood of future customer retention, or future 
customer referrals and, ultimately, future net income. However, after a given level of customer service is established, investment decisions that allocate additional resources to customer service may not increase the likelihood of future customer retention, or future customer referrals because of non-linearity. Thus, beyond some threshold, additional investments in customer service may negatively influence current and future net income. In an attempt to avoid this problem incentive structures that maximize contemporaneous goal commitment relative to a forward-looking goal commitment are preferred over those that do not.

Forward-looking measures can provide individuals with performance feedback as they progress towards attainment of the contemporaneous goal (Latham and Seijts 1999). In a task setting of low complexity, forward-looking goals can facilitate management decisions even if they are not contracted upon. However, forward-looking incentives are not needed because of the low cognitive processing demands in this setting (Dess and Beard 1984). Because individuals are able to readily identify causal relationship between performance on the forward-looking and contemporaneous measures, they will attempt to attain the forwardlooking goals even when no direct incentives are provided for doing so (Webb 2004).

Incentive structures that encourage high levels of commitment to the contemporaneous goal relative to the forward-looking goals are expected to result in the selection of better investment decisions, which will result in better management performance (Earley et al. 1992). In hypothesis one, I predict that relatively high weights on incentives for contemporaneous goal attainment will result in higher levels of contemporaneous goal commitment, relative to that of forward-looking goals. In this section, I extend my predictions to include individuals' investment decisions. I expect that as the 
contemporaneous weighting in the incentive contract increases, individuals will exhibit higher levels of contemporaneous goal commitment and, as a result, will perform better. In other words, I expect that individuals' commitment to the contemporaneous goal, relative to that of the forward-looking goals, will mediate the relationship between incentive weights and performance. I predict that, in a low complexity task, individuals assigned incentive contracts with a higher contemporaneous weighting will perform better by selecting better investment decisions. The hypothesized effects in a low complexity task are depicted in Figures 4 and 5. The hypothesized effects of rewarding individuals for the attainment of forward-looking and contemporaneous goals, in a low complexity task, are as follows:

H2: In low complexity tasks, as the weighting of incentives rewarded for contemporaneous goal attainment increases, individuals will make better investment decisions. (Figure 4, Condition $C>B>A$ )

H3: In low complexity tasks, as the weighting of incentives rewarded for contemporaneous goal attainment increases, individuals commitment to the contemporaneous goal, relative to that of the forward-looking goal, will mediate the relationship between incentive weights and investment decisions. (Figure 5)

\subsection{High Complexity Environment}

As task complexity increases, individuals face higher information processing requirements (Dess and Beard 1984). To perform well individuals must learn about various management investment decisions and the consequences of their actions. As discussed in Chapter 2, in a complex task goals indirectly affect performance by encouraging the discovery, and use of, task relevant knowledge and strategies (Wood et al. 1990, Stajkovic and Luthans 1998). In a complex task setting, where the causal relationships between forward-looking and contemporaneous measures are less clear to individuals, having goals for both types of measures will help individuals learn successful investment strategies (Locke and 
Latham 2002, Latham and Seijts 1999). Performance relating to forward-looking goals can provide timely, cognitive feedback to individuals about their performance and enable them to adjust their investment decisions if necessary (Kessler and Ashton 1981; Balzer et al. 1989; Leung and Trotman 2005). The contemporaneous goal motivates individuals to repeat actions that, based on forward-looking results, are congruent with the long-term objectives of the organization (Fishbach et al. 2006).

Because both forward-looking and contemporaneous goals are important to making better investment decisions, individuals that commit to both types of goals are likely to perform better than those who commit primarily to the contemporaneous goal (Winters and Latham 1996; Latham and Seijts 1999; Seijts et al. 2004). The attainment of forward-looking goals provides positive feedback to individuals about their performance, which then increases individuals self-efficacy in their abilities to make appropriate management decisions (Wood and Bandura 1989). Individuals' increased self-efficacy will heighten their aspirations to meet the contemporaneous goal, and their perseverance towards the contemporaneous goal, even if some forward-looking goals are not attained (Bandura and Simon 1977, Latham and Seijts 1999, Heath et al. 1999). Therefore, to facilitate effective decision making in a complex task setting it is important that individuals commit to forward-looking goals.

However, in some cases, simply providing forward-looking goals without rewarding their attainment does not appear to motivate individuals to sufficiently commit to them (Kelly 2007; Farrell et al. 2008). In complex environments because the increased task complexity requires greater cognitive resources and imposes greater information processing demands, individuals do not attend to all information provided. Incentives rewarded only for contemporaneous goal attainment can divert individuals' attention away from the forward- 
looking information, even though it can provide them with insights on how to perform a task (Wood et al. 1990). As discussed in Chapter 2, in a complex task, individuals that are committed to forward-looking goals are more likely to learn task appropriate strategies (i.e., good investment decisions), and attain the contemporaneous goal (Wood et al. 2000, Webb 2004). Providing incentives for forward-looking goal attainment can be used to attract individuals' attention to the forward-looking measures, and increase forward-looking goal commitment (Locke and Latham 2002). If individuals experience some success in attaining the forward-looking goals, this should in turn strengthen their belief that they can attain the contemporaneous goals, given the causal link between them (Webb 2004). Moreover, as discussed in Chapter 2, individuals that more thoroughly process the cognitive feedback provided by forward-looking goals can develop a more accurate mental representation of the causal business model and make better investment decisions sooner and more frequently than individuals who do not (Balzer et al. 1989; Leung and Trotman 1995; Atkins et al. 2002, Wood et al. 2000).

As noted in Chapter 2, monetary incentives for goal attainment can positively affect goal commitment (Hollenbeck and Klein 1987; Locke et al. 1988). Similar to the reasoning in developing the first hypothesis, I expect that individuals with incentive contracts that reward forward-looking goal attainment will commit to the forward-looking goals more than if only contemporaneous goal attainment is rewarded. Individuals committed to the forwardlooking goals will use the cognitive feedback provided by them to create a more accurate mental representation of the causal business model. As a result they will perform better by making better investment decisions that allow the attainment of the contemporaneous goal sooner and more frequently than individuals provided with incentives only on the 
contemporaneous measures. Indeed, the assignment of an incentive contract based only on contemporaneous measures will negatively impact performance due to increased commitment to the contemporaneous goal, relative to forward-looking goal commitment. In other words, I expect that individuals' commitment to the contemporaneous goal, relative to the forwardlooking goals, will mediate the relationship between incentive weights and performance. The hypothesized effects in a high complexity task are depicted in Figures 6 and 7. The hypothesized effects of rewarding individuals for the attainment of forward-looking goals, in a high complexity task, are as follows:

H4: In high complexity tasks, individuals who are rewarded for the attainment of both forward-looking and contemporaneous goals will make better investment decisions than those who are rewarded for the attainment of only contemporaneous goals. (Figure 6, Conditions D and E > Condition F)

H5: In high complexity tasks, the effects on investment decisions of rewarding the attainment of both forward-looking and contemporaneous goals will be mediated by the individual's commitment to the contemporaneous goal, relative to that of the forward-looking goal. (Figure 7, Panel A)

\subsection{Limitations of Incentives on Forward-looking Goals}

Next, I examine a setting where incentive contracts have a non-zero weighting on forward-looking measures. In the previous section I hypothesize that, in a complex task setting, forward-looking goals and incentives are decision facilitating because they enable individuals to overcome the starting problem and make better investment decisions.

However, the decision facilitating effects of forward-looking goals are only beneficial when an individual also remains committed to contemporaneous goal attainment (Fishbach et al. 2006). If the individual's commitment to the contemporaneous goal is relatively low, forward-looking goals can become decision influencing, instead of decision facilitating 
(Fishbach et al. 2006, Camerer et al. 1997). In other words, individuals can become too focused on simply attaining the forward-looking goals, instead of thinking about them as a means of attaining the contemporaneous goals. Excessive focus on attaining forward-looking goals can lead to less effective investment decisions that may not result in contemporaneous goal attainment (Hsee et al. 2003; Fishbach et al. 2006). This effect occurs because individuals will make investment decisions to satisfy the requirements of the goals to which they are committed (Chesney and Locke 1991; Wood et al. 1987). In this section, I examine one type of inventive contract that may allow this to occur: contracts with a non-zero weighting on forward-looking measures.

As per the reasoning used in developing the first hypothesis, incentive contracts that do not weight the contemporaneous goals as heavily as the forward-looking goals (Figure 3, Condition D), will result in individuals being less committed to the contemporaneous goal than the forward-looking goals. I expect that these individuals will be less likely to identify investment decisions that meet the long-term objectives of the organization and performance will suffer. Conversely, I predict that individuals assigned an incentive contract where contemporaneous goals are rewarded more heavily than forward-looking goals (Figure 3, Condition E) will be more committed to the contemporaneous goal and, as a result, will perform better. I expect that individuals with higher levels of contemporaneous goal commitment will perform better by making better investment decisions. Finally, as discussed in the development of the hypotheses in the low complexity setting, I expect that goal commitment will mediate the relationship between incentives and performance.

The hypothesized relationships between incentive contract weights and performance on contemporaneous measures in a high complexity task are depicted in Figures 6 and 7. The 
hypothesized effects of rewarding individuals more heavily for attainment of forward-looking goals than contemporaneous goals, in a high complexity task, are as follows:

H6: In high complexity tasks, individuals will make better investment decisions when incentives rewarded for contemporaneous goal attainment are weighted more heavily than those for forward-looking goal incentives. (Figure 6, Condition $D<E$ )

H7: In high complexity tasks, when incentives are rewarded for both contemporaneous and forward-looking goal attainment, individuals' commitment to the contemporaneous goal, relative to that of the forward-looking goal, will mediate the relationship between incentives and investment decisions. (Figure 7, Panel B)

\subsection{Summary}

This chapter develops seven hypotheses based on theory drawn from psychology and accounting research. The overall objective of the hypotheses is to identify the incentive structures that motivate individuals to make better investment decisions in a manner consistent with the organization's long-term objectives in both high and low complexity tasks. 


\section{CHAPTER 4: RESEARCH METHOD}

\subsection{Design Overview}

To test the hypotheses, this study employs a 2 × $3 \times 36$ mixed factorial design with task complexity and incentive contract as between-subject factors, and period as a withinsubjects factor. Participants, assigned the role of restaurant manager, use a computerized business simulation to make resource allocation decisions.

This chapter is organized as follows. Section 4.2 describes the simulation used in the experiment. Section 4.3 explains the experimental design. Section 4.4 describes the dependent variables. This chapter concludes in Section 4.5.

\subsection{Simulation Details}

\subsubsection{Task}

The business simulation describes a hypothetical franchise outlet of a well-known, national steak house. Each participant is assigned the role of restaurant manager for a local franchise. Participants are informed that, as manager of the restaurant, they are exclusively in charge of spending on four dimensions of the business: customer service, food quality, menu variety and restaurant atmosphere. ${ }^{18}$ On a "monthly" basis, participants must decide how to allocate an assigned monthly expenditure budget to these areas. Participants' investment decisions impact performance on both forward-looking and contemporaneous measures. The use of a resource allocation task is a consistent with recent experimental research (Kelly 2007; Cardinaels 2008; Kelly 2009).

\footnotetext{
${ }^{18}$ In the low complexity condition participants are only responsible for two business areas: customer service and food quality.
} 
The experimental currency is Canadian Lira. At the end of the simulation, participants exchange their earnings from Lira into Canadian Dollars at an exchange rate of $\$ 1$ per 1,000 Lira. The use of experimental currency is consistent with recent accounting experiments (Fisher et al. 2003; Fisher et al. 2005; Kelly 2007) and allows participants to operate in a realistic business environment where the money (in Lira) available to invest in the business is proportional to the individuals' earnings (in Lira). For example, it is more realistic that mangers of a restaurant earn a monthly bonus of 300 monetary units, than 3 . Using an experimental currency allows the ratio between each manager's incentives, restaurant investments, and restaurant income to be plausible, while keeping the actual incentives paid to participants (in Canadian dollars) over the course of the experiment at a manageable amount.

\subsubsection{Forward-Looking Dimensions}

On a monthly basis, participants are asked to allocate a maximum of 40,000 Lira among up to four dimensions: customer service, food quality, restaurant atmosphere, and menu variety. ${ }^{19}$ Participants are told that: (1) customer assessments of the four dimensions are measured using survey information collected at the end of their dining experience; (2) customers provide their assessments of each dimension using an 11-point scale, ranging from extremely dissatisfied (0) to extremely satisfied (10); and (3) the average scores are reported at month's end. In the restaurant business, a customer's assessment of the four performance dimensions at the end of their dining experience can affect future sales levels (see Figure 8). Customers who are satisfied with their dining experience are more likely to return to the

\footnotetext{
${ }^{19}$ In the low complexity condition, participants are asked to allocated current resources to only two dimensions: customer service and food quality. In these conditions, participants are asked to allocate a total of 20,000 Lira between the two.
} 
restaurant in the future, or to recommend the restaurant to others (Berry 2001; O'Connell 2006; Ross 2007).

There are various factors that can affect each of the four forward-looking dimensions. For example, the attentiveness and timeliness of service can affect customer service; the freshness and quality of the ingredients used in food preparation can affect food quality; the cleanliness of the restaurant and the attire of the wait staff can affect restaurant atmosphere; and the number of different menu items and daily specials available affect menu variety. In the restaurant business, all four forward-looking measures can be influenced almost immediately with investments in additional resources related to these areas (O'Connell 2006; Ross 2007).

\subsubsection{Contemporaneous Measure}

Future sales are positively affected by current resource allocation decisions.

Consistent with observations in practice (Ittner et al. 1998), performance on the forwardlooking measures in this simulation is not strictly linearly related with future sales. ${ }^{20}$ After a certain point additional investment in a particular area, such as food quality, positively affects future sales at a decreasing rate. For example, Figure 9 depicts the relationship between current investments in food quality and future sales. Investments up to 9,000 Lira positively affect future sales, but investments above this amount do not. ${ }^{21}$ Because the best investment is less than the restaurant's total monthly spending budget, excess funds are available to invest elsewhere in the company. In this simulation, participants must consider the appropriate level of total investment.

\footnotetext{
${ }^{20}$ This feature of the simulation also makes the task more challenging for participants than if a strictly linear relationship between forward-looking measure performance and future sales was used.

${ }^{21}$ This relationship exists in both conditions of high and low complexity.
} 


\subsubsection{Goals}

All individuals are assigned forward-looking and contemporaneous goals. Forwardlooking goals (i.e. customer service, food quality, restaurant atmosphere and menu variety scores) are leading, non-financial, monthly targets. Contemporaneous goals are financial targets for monthly net income. To ensure that the participants remain aware of their assigned goals, goal information is accessible throughout the simulation.

Customer service, food quality, restaurant atmosphere and menu variety goals are assigned at the level of resource allocation required for the highest positive impact on future income. Specifically, the goal assigned for all forward-looking dimensions is a score of 7 out of 10. The forward-looking goals can be attained by several different resource allocations. For example, an allocation of 10,000 Lira to each of the four different areas results in attainment of all four forward-looking goals (see Table 1).

The contemporaneous goal is based on monthly net income. In the high complexity conditions, the goal assigned for monthly net income is 5,100 Lira, while in the low complexity conditions the goal is 3,100 Lira. The contemporaneous goal can be attained by several different resource allocations. For example, a resource allocation strategy of 2,000 Lira to customer service, 6,000 Lira to food quality, 7,000 Lira to restaurant atmosphere, and 4,000 Lira to menu variety results in net income goal attainment (see Table 2, Panel B). Importantly, only one resource allocation strategy results in the attainment of both forward-looking and contemporaneous goals. Specifically, a resource allocation strategy of 5,000 Lira to customer service, 9,000 Lira to food quality, 8,000 Lira to restaurant atmosphere, and 6,000 Lira to menu variety results in the attainment of the contemporaneous and forward-looking goals (see Table 2, Panel B). In this allocation strategy, participants only 
needed to invest 28,000 of the available 40,000 Lira per period. ${ }^{22}$ Investment in any of the dimensions in excess of the required investment will allow participants to continue to meet the forward-looking goals, but not the net income goal. This design feature was included for two reasons. First, the negative net income effects of investment in forward-looking dimensions above the optimal amount required reflects the non-linear nature between dimensions documented in prior studies (Ittner et al. 1998). Second, the chance to over invest in forward-looking dimensions provides an opportunity for the participants to act in a manner that benefits their own personal wealth, but does not benefit the overall organization's wealth $^{23}$. Over-investment in forward-looking measures would allow participants to exceed the forward-looking goals, but would have a negative impact on the company's current period net income. This feature of the design is similar to other effort shifting opportunities faced by employees (Meyer 2002; Smith 2002).

\subsubsection{Feedback on Performance}

Feedback available to participants on a monthly basis includes: customers' forwardlooking measure assessments, monthly net income, and monthly incentives earned. Consistent with Kelly (2007), participants are provided with a full history of their monthly resource allocations, restaurant performance, and individual incentives earned. Providing all participants with access to the same information throughout the simulation decreases the possibility that experimental results are caused by differences in information availability or participants' inability to recall previous allocation decisions.

\footnotetext{
${ }^{22}$ Participants were able to invest up to 40,000 Lira in any of the 4 dimensions, but the total investment across all dimensions was limited to 40,000 each period.

${ }^{23}$ Although participants could increase their wealth by investing more in the 4 dimensions, to maximize their earnings they must meet both the forward-looking and contemporaneous goals. In meeting both forward-looking and contemporaneous goals, the participant acts in a manner that is consistent with the organization's objectives.
} 


\subsubsection{Work Horizon of the Employee}

The experiment is designed to induce a long-term work horizon for all participants. In existing experimental research, researchers have created a short-term horizon by notifying participants that they are working for different companies in each period (Farrell et al. 2008), or creating uncertainty as to the number of periods of play (Kelly 2007; Kelly 2009). To create a long-term environment in this experiment, I employ opposite strategies to these shortterm horizon manipulations. Participants work for the same company for the entire simulation (also consistent with the long-term horizon manipulation of Farrell et al. (2008)), and are informed that the simulation lasts 36 periods. ${ }^{24}$ If participants were not informed of the simulation length, their motivation would be to maximize their own wealth immediately, in essence acting as though they had a short-term profitability horizon.

\subsubsection{Pilot Testing}

Prior to collecting the data used in this study, I conducted a series of four pilot tests to test various aspects of the simulation. First, I conducted a pilot test to ensure that various characteristics of the simulation (e.g., participant task, incentive contracts, and assigned goals) were functioning as intended. Then, I conducted three pilot tests to develop a complexity manipulation that would result in sufficient complexity differences between the high and low complexity conditions. The results of the pilot tests showed that the characteristics of the simulation, and the complexity manipulation functioned as intended.

\footnotetext{
${ }^{24}$ This design feature introduces the opportunity for participants to change their strategies at the end of the game in order to maximize their wealth. For example, to maximize net income in the final period of the game, participants should not invest in any of the four dimensions.
} 


\subsubsection{Participants}

Participants in this study are primarily undergraduate university students.

Undergraduate students are appropriate participants because it is relatively easy to understand the task requirements and the simulation does not require specialized industry or accounting knowledge to complete. The use of undergraduate students is consistent with other accounting studies where participants were required to make resource allocation decisions, quality versus quantity tradeoffs, and production decisions (Fredrickson et al. 1999; Sprinkle 2000; Towry 2003; Farrell et al. 2008; Schulz et al. 2008; Kelly 2009).

\subsection{Experimental Design}

I employ a 2 x 3 x 36 mixed factorial design with task complexity (two levels) and incentive contracts (three levels) as between-subject factors and period as a within-subjects factor. Figure 3 illustrates the design of the experiment. Upon arriving at the experiment site, participants log on to the computer simulation. The simulation program randomly assigns participants to one of the six experimental conditions or two control conditions described below.

\subsubsection{Task Complexity}

The first independent variable is task complexity. Participants are randomly assigned to a management task of low or high complexity. In the low complexity condition, individuals operate in an environment where only two forward-looking dimensions (customer service and food quality) affect net income, with a one period lag. 
To increase the complexity of the task, I modify two features of the simulation. ${ }^{25}$ First, in the complex task, participants are required to manage two additional dimensions, resulting in a total of four forward-looking dimensions that require resource allocation decisions. The inclusion of additional dimensions in the business environment increases the amount of information individuals must consider, which in turn increases the complexity of the management task (Bonner 1994; Pelham and Neter 1995; Winters and Latham 1996). Second, the temporal relationship between forward-looking investments and future sales was altered to increase the task complexity. In the high complexity condition, a three period lag is used between forward-looking investments and sales. The use of lagged information is described in the dynamic decision making literature as an effective way to increase the complexity of an experimental task (Sternman 1989; Brehmer 1996; Dhaliwal and Benbasat 1996).

\subsubsection{Performance Incentives}

The second independent variable is the nature of the performance incentives contract. Participants are rewarded for the attainment of forward-looking (e.g., customer service) goals and/or the contemporaneous (i.e., net income) goal. Incentives earned for customer service, food quality, restaurant atmosphere, menu variety and net income goal attainment are based on monthly performance.

In creating the incentive conditions, I sought to create a plausible structure that is generally consistent with research-based evidence and observations from practice. The factors I considered when developing the incentive contracts are as follows. First, research

\footnotetext{
${ }^{25}$ Pilot testing results indicated that neither manipulation on its own was sufficient to significantly increase the complexity of the task.
} 
shows that the relationship between investments in customer service, food quality, restaurant atmosphere, and menu variety and future net income is not always linear (i.e., extremely high levels of customer service are not likely to result in significantly better contemporaneous performance than moderately high levels of customer service would) (Ittner et al. 2002). Thus, individuals should not be rewarded for maximizing forward-looking measures, while neglecting contemporaneous measures. Second, the primary performance objective is to maximize net income (i.e., individuals are rewarded for meeting or exceeding net income goals) (Smith 2002, Farrell et al. 2008). Third, to encourage individuals to attend to all forward-looking dimensions, they are assigned a minimum performance threshold (i.e., individuals must attain a minimum score of 3 on all measures to be eligible for any bonus on any indicator) (Meyer 2002).

In addition to a flat wage of 300 Lira for each month of work, participants in all incentive conditions could earn a maximum incentive payment of 300 Lira per month (see Table 3) if they attained all assigned goals. ${ }^{26}$ The opportunity to earn the same amount across conditions, establishes a consistent amount of available compensation.

The allocation of the 300 Lira maximum monthly bonus available between the contemporaneous and forward-looking goals varies across conditions. Specifically, three different weightings of the contemporaneous and forward-looking goals are employed. In the first condition ("low"), the contemporaneous goal receives a weighting of $1 / 3$ the maximum total incentive available with the remaining $2 / 3$ allocated to forward-looking goal attainment. In the second condition ("medium"), the contemporaneous goal receives a weighting of $2 / 3$

\footnotetext{
${ }^{26}$ This compensation structure reflects commonly used contracts in industry, where individuals total compensation package includes a fixed wage and a variable incentive, contingent on performance levels (Indjejikian and Nanda 2002; Banker et al. 2000).
} 
with the remaining $1 / 3$ allocated to forward-looking goal attainment. Finally, in the third condition ("high") the entire bonus is allocated to attaining the contemporaneous goal.

Consistent with the organizational objective to maximize net income, individuals are further rewarded if their performance exceeds the assigned net income goal. In the low (high) complexity condition individuals are rewarded an additional bonus if monthly net income exceeds 4,999 $(6,999)$ Lira. Similarly, in conditions where forward-looking goal attainment is rewarded, individuals receive additional compensation if they attain a score of 8 or higher in any of the forward-looking measures. This feature, designed to provide an incentive for participants to attain performance above their assigned goals, is common in organizations (Indjejikian and Nanda 2002; Ittner et al. 2003). Similar to a budget-linear incentive contract, this contract is expected to motivate participants to meet and, if possible, exceed the assigned goals (Sprinkle 2000). For all goals assigned, participants are offered a bonus for goal attainment, and a $20 \%$ larger bonus for exceeding the assigned goal. The amount of $20 \%$ is consistent with observations in practice (Indjejikian and Nanda 2002). ${ }^{27}$ This feature of the design forces participants to make a decision between investing more in forward-looking dimensions (to positively influence the forward-looking bonus) and investing less in forwardlooking dimensions (to decrease monthly expenditures and positively influence net income). ${ }^{28}$ In this simulation, participants are faced with a trade-off between improving performance in forward-looking and contemporaneous measures.

\footnotetext{
${ }^{27}$ The $20 \%$ increase in bonus was selected because it is large enough to entice participants to consider exceeding the assigned goal, but small enough that it was less than the payment for attaining other goals. (As described in section 4.2.4, exceeding the forward-looking goal is likely to result in failure to attain the contemporaneous goal.)

${ }^{28}$ As described in section 4.2.4, the participant must attain both the forward-looking and contemporaneous goals to maximize the total bonus earned.
} 


\subsubsection{Control Conditions}

To determine a baseline level of performance, without the effects of goal based incentive structures, two control conditions are included in the experimental design. In both the high and low task complexity control conditions, participants are assigned forwardlooking and contemporaneous goals, but are not rewarded with any goal-based incentives. Instead, they are simply paid a flat wage of 600 Lira per period. 600 Lira was selected because it is the total of the flat wage of 300 Lira, and the maximum available for goal based performance of 300 Lira in the other conditions.

\subsection{Dependent Variables}

\subsubsection{Goal Commitment}

At the end of periods 12 and 24, I measure contemporaneous and forward-looking goal commitment. Goal commitment is measured using the goal commitment scale created by Hollenbeck et al. (1989) and adapted by Klein et al. (2001). Goal commitment responses are collected using a 7-point Likert scale ranging from -3 (Strongly Disagree) to +3 (Strongly Agree). The questions used in this study are presented in Table 4.

To control for potential order effects of the forward-looking and contemporaneous goal commitment questions, the order of commitment question type is randomized between participants. Some participants answer forward-looking commitment questions first followed by contemporaneous questions, while the others answer contemporaneous commitment questions first, followed by forward-looking commitment questions. 


\subsubsection{Investment Decisions}

The quality of the investment decisions is directly reflected in the restaurant's net income. In the simulation, the amount of net income earned by participants is directly related to the quality of participants' investment decisions. For example, a relatively high net income balance indicates that the participants considered both forward-looking and contemporaneous measures performance when making investment decisions. ${ }^{29}$ In addition, net income provides a combined measurement of the participants' decisions to invest in forward-looking measurements. Thus, in my setting, net income provides a cumulative measure of both forward-looking and contemporaneous performance.

In the high complexity condition, due to the lagged effects of resource allocations on gross income, the first three months of income is not affected by the participants' decisions. Gross income only begins to fluctuate after the fourth period. Therefore performance in the high complexity tasks is measured using net income results in periods 4 through 36 . In the low complexity tasks, the shorter time lag means that only the first month is not affected by participants' decisions. Therefore performance in the low complexity tasks is measured using net income results in periods 2 through 36.

\subsubsection{Complexity Manipulation Check}

At the end of periods 12, 24 and 36, participants answer several questions to permit an assessment of the manipulation of task complexity. In total ten questions are asked: four questions are adapted from Wood's Task Complexity Scale (Wood 1986), and six additional

\footnotetext{
${ }^{29}$ As described in Section 4.2.4, participants must attain forward-looking and contemporaneous goals in order to earn relatively high levels of net income.
} 
questions were developed. ${ }^{30}$ Responses are collected using a 7-point Likert scale ranging from -3 (Strongly Disagree) to +3 (Strongly Agree). The questions used in this study are presented in Table 5.

\subsubsection{Task Understanding}

I use two methods to establish task understanding by participants. First, participants are required to complete a short series of multiple-choice and short answer questions.

Participants are asked the questions after reading the description of their role as restaurant manager, but prior to completing the practice round, or investment rounds. The questions are designed to reinforce participants' understanding of the key components of the experiment. Depending on the assigned condition, participants are asked up to eight questions ${ }^{31}$. The questions address the employment horizon, total resources available for monthly investment, forward-looking and contemporaneous goal assignments, and forward-looking and contemporaneous goal based incentives. The simulation informs participants immediately if they answer a question incorrectly. In order to proceed to the next question (and ultimately begin managing the restaurant) participants are required to provide the correct answer to each question. To assist them with this task, participants are able to access all of the simulation information at any time. The questions and answers are included in Table 6. Second, participants practice their task before commencing the simulation. All participants complete one practice period to familiarize themselves with the user interface before beginning period 1

\footnotetext{
${ }^{30}$ Five of the additional complexity questions were added to the simulation partway through the data collection process.

${ }^{31}$ Some questions do not apply to certain conditions. For example, in conditions where participants are not assigned incentives for forward-looking goal attainment, the short answer question regarding this topic is omitted from the question set.
} 
of the simulation. Inclusion of a practice period in the experimental design is consistent with prior research (Libby 2001; Fisher et al. 2003; Sprinkle 2003).

\subsubsection{Controls and Other Measured Variables}

To control for academic experience, participants are asked to disclose their program of study, their academic year, and the number of accounting courses taken. This measure was taken to control for any differences across conditions in participants' business and accounting knowledge. To control for any industry experience effects, participants are asked if they have ever worked in the restaurant business and, if so, to provide their job title and years of experience.

To guard against various perceptions about the simulation's characteristics affecting the experimental results, I ask the participants three questions about the task. At the end of the simulation, participants are asked to assess whether the simulation task was realistic, easy to understand, and interesting. Responses are collected using a 7-point Likert scale ranging from -3 (Strongly Disagree to 3 (Strongly Agree).

To control for participants' prior beliefs about the restaurant industry, that might affect their decisions, two methods are employed. First, after learning about the four forwardlooking dimensions, but before learning about the goals or incentive structures, participants are asked about their perceptions of the importance of the forward-looking dimensions. Similar to the approach used by Kelly (2007), participants were asked to allocate 100 points among the four dimensions to indicate their relative importance in this industry. (For example, allocating 25 points to each investment would indicate that the participant believes all four forward-looking dimensions are equally important to a restaurant's success.) Second, 
participants are told that the previous restaurant manager invested equally (i.e., 10,000 Lira) in each area of the restaurant on a monthly basis. Participants are also told that the company is not sure if this is the best way for individuals to allocate their budget ${ }^{32}$. This information was presented to provide all participants with the same starting point for resource allocations.

To guard against participants sharing results between administrations of the various sessions, two different actions are taken. First, all participants are asked to sign an agreement of confidentiality confirming that they will not share the details of the experimental task with others. Second, in the low complexity condition, three different optimal resource allocation schemes are randomly assigned to participants. ${ }^{33}$ In the first condition, introduced earlier in this chapter, the optimal monthly investment is 5,000 Lira to customer service and 9,000 Lira to food quality (See Table 1). In the second condition an allocation of 9,000 Lira to customer service and 6,000 Lira to food quality results in the highest future net income levels. In the third condition the optimal allocation is 8,000 Lira to customer service and 6,000 Lira to food quality. All other allocations result in a lower net income. ${ }^{34}$ Consistent with the relationships shown in Table 1, each optimal investment generates a positive cumulative net income effect of 1,000 Lira.

\footnotetext{
${ }^{32}$ Monthly investments of 10,000 Lira in each forward-looking measure is the basis of the results for the first periods of business in the restaurant, before the lagged effects of participants' investment decisions impact restaurant sales (period 2 in low complexity condition, period 4 in high complexity condition). This feature of the design avoids the use of deception because participants where informed that the previous manager invested 10,000 Lira in each forward-looking measure on a monthly basis.

${ }^{33}$ A similar strategy was not used in the high complexity condition. Based on observations made during pilot testing, participants in the high complexity condition were less likely to identify all four optimal investments over the course of the experiment. Since few participants discovered the optimal allocation strategy in the high complexity condition, the risk that new participants would be informed of the optimal investment strategies prior to commencing the simulation was low.

${ }^{34}$ For simplicity, throughout the remainder of this study, I only refer to the 5,000/9,000 allocation condition when discussing the low complexity condition.
} 


\subsection{Summary}

A $2 \times 3 \times 36$ mixed factorial experiment is employed to test whether task complexity and incentive structures affect individuals' decisions over 36 periods. Profitability of the

restaurant is used to evaluate the individuals' performance. A summary of the experimental procedures is presented in Table 7 . The next chapter discusses the results of this experiment. 


\section{CHAPTER 5: RESULTS}

\subsection{Introduction}

This chapter provides results of the experiment. Section 5.2 reports the demographic information about the participants. Section 5.3 examines the manipulation checks and Section 5.4 reports the analysis of the control variables. The effect of incentives on goal commitment (Hypothesis 1) is tested in Section 5.5. The relationship between goal-based, performance incentives and investment decisions in the low complexity conditions (Hypothesis 2 and 3) are tested in Section 5.6. The effect of incentives on investment decisions in high complexity conditions are considered in Section 5.7 (Hypotheses 4 and 6) and Section 5.8 (Hypotheses 5 and 7). Control conditions are analyzed in Section 5.9. The chapter concludes in Section 5.10 .

\subsection{Demographic Information about Experimental Participants}

In total, 279 students participated in this study. Eight participants had technical difficulties part way through their first round with the simulation. These students were allowed to restart the simulation and, in all cases, completed the study without further problems. In all eight cases the simulation did not record the participant's performance from their first attempt. The participants' performance on their second attempt of the simulation was recorded, but removed from my analysis. I removed this data because these participants had more knowledge about the simulation after starting over and likely had an advantage over the other participants in finding the "best" investments in forward-looking measures sooner. An additional 7 observations are eliminated from the data. In five of the eliminated observations, participants' responses demonstrated a significant lack of understanding 
regarding the parameters of the simulation. Specifically, the participants allocated very small amounts (i.e., less than 100 Lira) to each forward-looking measure for many of the periods of the simulation. The remaining two participants completed the experiment in less than 10 minutes, and began the simulation with the correct resource allocation amounts. These observations suggest that these participants were informed of simulation details prior to commencing the experiment. In total, 15 observations (5.38\% of the original sample) were removed from the original sample. I perform all analysis in this chapter using the final sample of 264 observations.

Table 8 provides background information about the 264 participants in this study. Participants were recruited from third and fourth year undergraduate accounting courses. Students registered in these classes received an invitation to participate in my experiment via e-mail. In addition, I visited class to invite participants to attend my experiment. Experimental sessions were held in a computer lab, outside of class time.

On average, participants spent 28 minutes on the task, and earned $\$ 18.70$. Participants ranged from first year to fourth year undergraduate students, with $93 \%$ of students in their third or fourth year of study. Fifty-eight percent of participants were working towards an accounting degree. The program year and degree do not vary significantly between experimental conditions (program year: $X^{2}=1.20, p=0.99$, two tailed; degree: $X^{2}=15.05, p$ $=0.38$, two tailed, results not tabulated). At the time of the experiment participants had completed an average of nearly eight accounting courses. The number of accounting courses does not vary significantly between experimental conditions $(F=0.35, p=0.93$, two tailed, results not tabulated). Nineteen percent of participants had restaurant work experience. Of those with restaurant experience, the average length of employment is slightly more than one 
year. The number of participants with restaurant experience and the average length of restaurant employment do not significantly vary between conditions (restaurant experience: $\mathrm{X}^{2}=4.21, \mathrm{p}=0.76$, two tailed; length of employment $\mathrm{F}=0.99 \mathrm{p}=0.45$, two tailed, results not tabulated).

\subsection{Task Complexity Manipulation Check}

As discussed in Section 4.4.4, participants were asked ten different complexity manipulation check questions. Participants' responses to these questions are used to check the manipulation of low and high task complexity. During the first administration of this experiment, only five complexity questions were asked and this was done at the end of period 36. In reviewing the results from the first administration, I observed that at the end of the experiment participants' perceptions about task complexity were affected not only by the actual task, but also their ability to learn the task. In this study, I hypothesize that task learning will be affected by the incentive contract assigned. Therefore, in order to measure the participants' perceptions of complexity, as generated by task characteristics, I added five new questions to the second administration of the simulation. Three new questions were asked during the simulation, at the end of periods 12 and 24, and two new questions were asked at the end of the simulation.

The mean participant responses to the first three questions, asked at the end of periods 12 and 24, are shown in Table 9, Panel A. Because the responses are all highly correlated (Table 9, Panel B), and each participant was asked the same questions twice during the experiment, I perform a repeated measures multivariate analysis of variance. The results of this analysis (see Table 9, Panel C) show that, between subjects, the task complexity 
manipulation significantly affects perceptions of complexity $(\mathrm{F}=2.58, \mathrm{p}=0.06$, two tailed).

There is also a significant difference between the first and second period in which the questions were asked $(\mathrm{F}=2.31, \mathrm{p}=0.08$, two tailed). Review of the descriptive statistics (Table 9, Panel A) shows that, overall, participants' assessments of complexity were lower in period 24, than in period 12. Thus, it appears that as the simulation progressed, participants found their task to be less difficult. Further analysis of each question shows that the task complexity manipulation significantly affects participant perception of task difficulty related to net income (i.e., contemporaneous) performance (Table 9, Panel D: F = 5.27, p = 0.02, two tailed) and overall task difficulty (Table 9, Panel F: $F=6.44, p=0.01$, two tailed), but not the nonfinancial (i.e., forward-looking) performance (Table 9, Panel D: $F=2.06, p=0.15$, two tailed). The descriptive statistics in Table 9, Panel A show that participants in the high complexity condition agree more strongly that attainment of the net income goal (e.g., Period 12 mean score $=0.58)$ is difficult than those in the low complexity condition (e.g., Period 12 mean score $=-0.35)^{35}$. Similarly, participants in the high complexity condition agree more strongly that the overall task of managing the restaurant is difficult (e.g., Period 12 mean score $=0.35$ ) than those in the low complexity condition (e.g., Period 12 mean score $=-0.35$ ). ${ }^{36}$ Participants' assessments of task complexity are not affected by incentive condition (Panel C, $\mathrm{F}=0.05, \mathrm{p}=0.59$, two tailed).

The mean participant responses to the remaining seven complexity questions, asked at the end of period 36 are reported in Table 10, Panel A. Because participant responses to the seven questions are highly correlated (Table 10, Panel B) I perform a multivariate analysis of variance. When the analysis includes all seven questions, results show that complexity

\footnotetext{
${ }^{35}$ This difference is significant $(\mathrm{p}<0.01$, one tailed, not tabulated).

${ }^{36}$ This difference is significant $(\mathrm{p}<0.01$, one tailed, not tabulated).
} 
manipulation significantly affects participants' perceptions about complexity (Table 10, Panel $\mathrm{C}: \mathrm{F}=3.79, \mathrm{p}<0.01$, two tailed), but incentives condition does not $(\mathrm{F}=0.90, \mathrm{p}=0.60$, two tailed). A MANOVA of the original five questions also shows that complexity and incentives both significantly affect participants' responses to the questions (Table 10, Panel D: Complexity: $F=4.94, p<0.01$, two tailed; Incentive: $F=1.79, p=0.03$, two tailed). ${ }^{37}$ Variance analysis, by question, shown in Table 10, Panel E, shows that both complexity and incentives are significant in four of the five original complexity questions (questions 4, 5, 7, and 8). As shown in the descriptive statistics by question (Table 10, Panel A), participants in the high complexity condition agree more strongly that the task is difficult (e.g., Question 4 mean $=0.27)$ than those in the low complexity condition (e.g., Question 4 mean $=-0.45)$. Incentives has a significant main effect in this analysis because for questions 4, 5, 7 and 8 participants in the low contemporaneous weighting condition indicate that they found the task to be more difficult than those in the high contemporaneous weighting condition. Review of the descriptive statistics in Table 10, Panel A shows that this pattern is similar in both complexity conditions. For each of the questions added to the end of the simulation in the second administration (questions 9 and 10) the main effect of complexity is significant (Table 10, Panel E: Question 9, F = 9.35, p < 0.01, two tailed; Question 10, F = 18.16, p $<0.01$, two tailed), but the main effect of incentive is not significant.

The multivariate analysis of the full sample (Table 10, Panel D) shows a significant interaction between complexity and incentives $(\mathrm{F}=1.78, \mathrm{p}=0.03$, two tailed). Individual question analysis (Table 10, Panel E) shows that the significant interaction arises in questions $7(\mathrm{~F}=4.20, \mathrm{p}<0.01$, two tailed $)$ and $9(\mathrm{~F}=2.63, \mathrm{p}=0.05$, two tailed $)$. In both cases, the

\footnotetext{
${ }^{37}$ This analysis is also performed with experiment administration (i.e., first or second) as an additional between subjects factor. In all cases, the effect of administration on the complexity variables is not significant, and does not affect the reported results (not tabulated).
} 
interaction arises because complexity assessments varied between incentive conditions differently in the high and low complexity conditions. For example, complexity assessments of participants in the low complexity condition in question 7 are consistent with the nature of the incentives main effect observed above. Specifically, those in the low contemporaneous weighting condition found the task to be significantly ( $\mathrm{p}=0.09$, one tailed, not tabulated) less complex $($ Table 10, Panel A: mean $=0.70)$ than those in the high contemporaneous weighting condition (mean $=1.03)$. However, in the high complexity condition participants' assessments of complexity were not significantly different (low contemporaneous weighting mean $=1.08$, high contemporaneous weighting mean $=1.09$, p-value $=0.49$, one tailed, not tabulated). For question 9, the assessments of complexity in the high complexity condition are consistent with the pattern observed in analysis of the main effect of incentives on complexity assessments: those in the low contemporaneous weighting condition found the task to be significantly $(\mathrm{p}$-value $=0.09$, one tailed, not tabulated) less complex $($ mean $=1.00)$ than those in the high contemporaneous weighting condition (mean $=1.62)$. However, this pattern is not observed in the low complexity condition. Participants in the low contemporaneous weighting condition found the task to be significantly ( $\mathrm{p}=0.08$, one tailed, not tabulated) more complex $($ mean $=0.94)$ than those in the high contemporaneous weighting condition $($ mean $=0.14)$.

Overall, I find that the complexity of the task has a significant main effect on participants' assessments of complexity both during and upon completion of the simulation. However, I also find that the incentive contract assigned has a significant effect on participants' complexity assessments. As discussed in Chapter 3, I expect that the incentive contracts assigned in this simulation will either encourage, or limit, the participants' opportunity to learn good investment strategies. At the end of the experiment, those who 
identified good investment strategies are more likely to find the task to be less complex, than those who did not identify good investment strategies. Therefore, at the end of the simulation, participants' perceptions of task complexity are affected not only by task characteristics (e.g., number of forward-looking dimensions) but also their ability to learn good investment decisions, which are influenced by the incentive contract assigned. Thus, the effect of incentives on complexity judgments at the end of the experiment meets my expectations.

Kruskal-Wallis non-parametric tests, comparing the participants' responses to each question between complexity and incentive conditions are presented in Table 11. The results are generally consistent with the findings reported in the parametric tests.

As described in section 4.4.5, participants in the low complexity condition are assigned one of three possible sets of two forward-looking variables to manage. Repeated measures multivariate analysis results (Table 12, Panel A) and multivariate analysis results (Table 12, Panel B) show that participants' perceptions of task complexity are not significantly affected by the forward-looking measure set assigned (Table 12, Panel A: F= $0.21, p=0.89$, two tailed; Panel B: $F=1.81, p=0.12$, two tailed). In all analyses involving the low complexity condition, I include measure set as an additional between subjects factor. Unless otherwise noted, measure set does not have a significant effect on the results.

Based on the analysis of the ten complexity questions asked of participants, the complexity manipulation appears to have worked as intended. 


\subsection{Control Variables}

\subsubsection{Simulation Characteristics}

Participants' perceptions about the simulation characteristics are summarized in Table 13, Panel A. Overall participants believed the case was interesting (mean $=1.69$, on a -3 to 3 scale) and easy to understand (mean $=1.59$, on a -3 to 3 scale). On average, participants found the simulation to be slightly unrealistic (mean $=-0.15$, on a -3 to 3 scale). Because, all three measures are significantly correlated (Table 13, Panel B) I perform a multivariate analysis of variance. In this analysis, participant responses to each of the three questions are the dependent variables, task complexity and incentive contracts are the independent variables, and experiment session (i.e., first or second administration) is the between subjects factor. As shown in Table 13, Panel $\mathrm{C}$ the simulation characteristics do not vary significantly between complexity $(\mathrm{F}=0.75, \mathrm{p}=0.53$, two tailed $)$ and incentive conditions $(\mathrm{F}=0.64, \mathrm{p}=$ 0.77, two tailed). Non-parametric tests (Table 13, Panel D) for each question show similar results. Overall, it appears that participant's perceptions of simulation characteristics are not significantly affected by complexity or incentive condition.

In this analysis experiment session has a significant effect on participants' perceptions about the simulation characteristics (Table 13, Panel C: $F=3.29, p=0.02$, two tailed). Although there is not a significant difference in participants' assessments of the simulation's realism between sessions $(\mathrm{F}=1.81, \mathrm{p}=0.18$, two tailed, not tabulated $)$ a significant difference occurs in participant assessments of their ability to easily understand the task $(F=4.68, p=$ 0.03 , two tailed, not tabulated) and the degree to which the simulation is interesting $(\mathrm{F}=8.23$, $\mathrm{p}<0.01$, two tailed, not tabulated). In both questions, participants' responses are more positive in the second session (Question 2: Session 1 mean $=1.45$, Session 2 mean $=1.75$; 
Question 3: Session 1 mean $=1.52$, Session 2 mean $=1.89$, not tabulated). The difference in participant perceptions between experiment sessions may have an effect on their performance in the simulation. ${ }^{38}$ However, in both sessions, the means are significantly greater than the scale midpoint of 0 (understanding: p-value $<0.01$, two-tailed; interesting: $p$-value $<0.01$, two tailed; results not tabulated). This indicates that all participants found the case to be relatively easy to understand and interesting.

\subsubsection{Perceptions About Importance of Forward-Looking Measures}

Descriptive statistics for participants' perceptions about how the forward-looking measures contribute to a restaurant's success are shown in Table 14, Panel A. Inspection of the results shows that in both the low and high complexity conditions, participants indicated that food quality is the most important forward-looking measure. I analyze this data to ensure that there are no significant differences in perceptions regarding forward-looking measure importance among conditions. Because the perceptions of forward-looking measure importance are significantly correlated in both the low and high complexity conditions (all pvalues $<0.01$, two-tailed, not tabulated) I perform a multivariate analysis of variance (Table 14, Panel C). I find no significant between group differences in forward-looking measure importance for either the low $(\mathrm{F}=0.79, \mathrm{p}=0.51$, two tailed $)$ or high $(\mathrm{F}=0.57, \mathrm{p}=0.83$, two tailed) complexity conditions. ${ }^{39,40}$

\footnotetext{
${ }^{38}$ To address this concern, in all analyses, I include experiment session as an additional between subjects factor. Unless otherwise reported, experiment session did not significantly affect results.

${ }^{39}$ For both high and low complexity conditions, I perform additional analyses where experiment session (i.e., first or second administration) is included as an additional between-subjects variable. Experiment session does not have a significant effect on forward-looking importance perceptions (results not tabulated).

${ }^{40}$ Non-parametric tests (Table 14, Panel D) show similar results.
} 


\subsection{Goal Commitment}

Before testing hypotheses relating to goal commitment, I examine the reliability of the commitment scale used. Commitment is measured for forward-looking goals and contemporaneous goals at the end of periods 12 and 24. As shown in Table 15, Panel B, high factor loadings (i.e., greater than 0.60) suggest that each of the five factor loadings is significant (Stevens 1996). Further, Cronbach's alpha is above 0.80 for all four commitment measurements indicating that the commitment scale used is reliable (Diekhoff 1992).

Therefore, since all five factors appear to measure the same construct, I calculate a mean goal commitment score to use in the remainder of the analysis.

The order of forward-looking and contemporaneous commitment questions was randomized between participants. Some participants answered forward-looking questions first followed by contemporaneous questions, while the others answered contemporaneous questions first, followed by forward-looking questions. To examine the effect of order on goal commitment assessments, I perform a repeated measures MANOVA, where the within subjects, repeated measure is average forward-looking and contemporaneous goal commitment scores at periods 12 and 24 (Table 16, Panel B). The between subjects variables are question order, incentives and complexity. The results show that between subjects question order has a significant main effect on goal commitment ( $\mathrm{p}=0.07$, two tailed), but question order does not significantly interact with incentives ( $\mathrm{p}=0.68$, two tailed) or complexity ( $\mathrm{p}=0.42$, two tailed) to predict goal commitment. ${ }^{41}$ Post hoc analysis shows that

\footnotetext{
${ }^{41}$ The results also show that, between subjects, incentives (Table 16, Panel B: $p<0.01$, two tailed) and complexity $(\mathrm{p}<0.01$, two tailed) significantly affect goal commitment levels and, within-subjects, significant interactions between period and incentives ( $\mathrm{p}=0.02$, two tailed) and period, incentives and complexity ( $\mathrm{p}=0.05$, two tailed) are observed. The relationship between these variables and the goal commitment difference score is discussed in detail in Section 5.5.1.
} 
the significant relationship between question order and goal commitment is attributable to the significant difference in average contemporaneous goal commitment (Table 16, Panel C:

$p=0.05$, two tailed) but not the forward-looking goal commitment ( $p=0.63$, two tailed).

Review of descriptive statistics shows that participants who were asked contemporaneous goal commitment questions first responded with lower average contemporaneous goal commitment scores (Table 16, Panel A: mean contemporaneous goal commitment = 1.09) than those asked forward-looking goal commitment questions first (mean contemporaneous goal commitment $=1.39$ ). Given the significant main effect observed in this test, I perform additional analysis to include question order as a between subjects factor. Unless otherwise disclosed, question order has an insignificant effect on any analysis that includes goal commitment as a variable.

\subsubsection{Test of Hypothesis 1}

In Hypothesis 1, I posit that as the weighting of incentives rewarded for contemporaneous goal attainment increases, individuals commitment to the contemporaneous goal, relative to that of the forward-looking goal will increase. To test the hypothesis, I compare how commitment levels vary between forward-looking and contemporaneous goals. To perform this analysis I calculate a difference score, measuring the difference between contemporaneous and forward-looking goal commitment (see raw means, Table 17, Panel A). The difference score allows me to examine how, within subjects, commitment levels vary between contemporaneous and forward-looking goals.

The descriptive statistics presented in Table 17, Panel B show that when the incentive contracts weights contemporaneous goal attainment less than forward-looking goal attainment 
(i.e., low contemporaneous weighting) a negative difference score results. A negative difference score means that participants exhibited higher commitment levels to the forwardlooking goals, than the contemporaneous goals. The results also show that in incentive contracts with a high contemporaneous weighting, a positive difference score is observed. A positive difference score means that participants exhibited higher commitment levels to contemporaneous goals, than forward-looking goals.

To test the statistical significance of these observations, I perform a repeated measures analysis of variance, where the difference scores for periods 12 and 24 are the within subjects measure with incentives and complexity as the between subjects factors. ${ }^{42,43}$ Consistent with my expectations, the results show that incentives significantly (Table 17, Panel C: $F=7.77, p$ $<0.01$, two tailed) impacts participants' commitment to the contemporaneous goal relative to the forward-looking goal. Examination of the significant interaction between period and complexity (Table 17, Panel C: $\mathrm{F}=4.07, \mathrm{p}=0.05$, two tailed) shows that those in the high complexity condition, on average, had a higher increase in their difference score (Table 17, Panel B: Period 12 Mean $=-0.29$, Period 24 Mean $=0.14$ ) over time than those in the low complexity condition $($ Period 12 Mean $=-0.05$, Period 24 Mean $=0.03) .{ }^{44}$

Within subjects, the significance of period (Table 17, Panel C: $\mathrm{F}=8.32, \mathrm{p}<0.01$, two tailed) suggests that commitment difference scores are affected by the length of time the participants had been participating in the simulation. Overall, participants had a higher

\footnotetext{
${ }^{42}$ I performed a second analysis (not tabulated) including experimental session (i.e., first or second administration) as an additional between subjects factor. Experiment session did not significantly the results of this analysis.

${ }^{43}$ Participants in the control condition are not asked any of the goal commitment questions. Therefore, this analysis only includes those in the low, medium and high contemporaneous incentive conditions.

${ }^{44}$ Repeated measures analysis of variance confirms the nature of this interaction (Table 17, Panel D). In the analysis of low complexity, the goal difference scores are not significantly different over time (within subjects period effect: $\mathrm{p}=0.42$, two tailed) while in the analysis of high complexity goal difference scores are significantly different over time (within subjects period effect: $\mathrm{p}<0.01$, two tailed).
} 
difference score in the second measurement (Table 17, Panel B: Period 24, Mean =0.08) than in the first measurement (Period 12, Mean $=-0.17)$. This result indicates that participants became more committed to the contemporaneous goal as the simulation progressed. The significant interaction between period and incentive (Table 17, Panel C: $\mathrm{F}=3.07, \mathrm{p}=0.05$, two tailed) indicates that those in the low contemporaneous weighting condition (Table 17, Panel B: Period 12 Mean $=-0.62$, Period 24 Mean $=-0.29)$ and medium contemporaneous weighting condition $($ Period 12 Mean $=-0.24$, Period 24 Mean $=0.21)$ had a larger increase in their scores over time than those in the high contemporaneous weighting condition (Period 12 Mean $=0.39$, Period 24 Mean $=0.34) .{ }^{45}$

To test Hypothesis 1, I compare commitment levels amongst the three incentive conditions. ${ }^{46}$ In the repeated measures analysis of variance, incentives significantly affect goal difference scores (Table 17, Panel C: $\mathrm{p}<0.01$, two tailed). Thus, I find preliminary evidence to support my hypothesis that contemporaneous goal commitment relative to forward-looking goal commitment is affected by the weighting of incentives rewarded for the contemporaneous goal.

To test for the pattern of means examined by Hypothesis 1, I use contrast coding where I use a code that mimics the expected pattern of means (Buckless and Ravenscroft,

\footnotetext{
${ }^{45}$ Repeated measures analysis of variance confirms the nature of this interaction (Table 17, Panel E). In the analyses of the low and medium contemporaneous incentive contracts, the goal difference scores are significantly different over time (within subjects period effect, low contemporaneous weighting: $p=0.01$, two tailed; medium contemporaneous weighting: $p=0.01$, two tailed) while in the analysis of the high contemporaneous weighting incentive contract difference scores are not significantly different over time (within subjects period effect, high contemporaneous weighting: $\mathrm{p}=0.76$, two tailed).

${ }^{46}$ Consistent with Hypothesis 1 , goal commitment observations in both the high and low complexity conditions are tested in the same model. As expected, complexity does not significantly affect the commitment difference scores $(\mathrm{p}=0.62$, two tailed, Table 17, Panel C).
} 
1990; Drake et al. 1999). ${ }^{47}$ The commitment difference scores at period 12 and 24 are tested in separate models. The results of the contrast-coding models are presented in Table 17, Panel F. In both commitment difference scores, the results are highly significant (Period 12: $\mathrm{p}<0.01$, two tailed Period 24: $\mathrm{p}<0.01$, two tailed). Therefore, I find evidence consistent with Hypothesis $1 .^{48}$

Planned contrasts comparing the commitment difference scores among incentive conditions show that participants' contemporaneous goal commitment, relative to the forward-looking goal commitment significantly increases as the contemporaneous weighting increases from low to medium (Table 17, Panel G, Period $12 \mathrm{p}=0.05$; Period $24 \mathrm{p}=0.05$ ) and low to high (Table 17, Panel G, Period $12 \mathrm{p}<0.01$, one tailed; Period $24 \mathrm{p}=0.01$, one tailed) in both commitment measurements (Periods 12 and 24). For the first commitment measurement (Period 12) a significant difference exists between the difference scores of the medium and high conditions (from Table 17, Panel G: Period $12 \mathrm{p}=0.09$, one tailed), but this difference is not significant in the second commitment measures (Period $24 \mathrm{p}=0.50$, one tailed).

Overall, the results are generally consistent with Hypotheses 1 . The evidence shows that as the weighting of incentives rewarded for contemporaneous goal attainment increases, individual's commitment to the contemporaneous goal relative to that of the forward-looking goal increases.

\footnotetext{
${ }^{47}$ Contrast coding of $-0.5,-0.1$, and 0.6 is used for this test. Results similar to those reported occur when other weight patterns that are consistent with the expected cell mean patterns are used (e.g., $-0.5,0.1$ and $0.4 ;-1.5,0.5$, and $1.0 ;-1.5,-0.5$, and $2.0 ;-10.0,-0.5$, and $10.5 ;-10.0,0.5$ and 9.5$)$.

${ }^{48}$ Non-parametric tests (Table 17, Panel H) provide similar results.
} 


\subsection{Investments in a Low Complexity Task (Test of Hypotheses 2 and 3)}

\subsubsection{Test of Hypothesis 2}

In Hypothesis 2, I predict that in low complexity tasks, individuals will make better investments as the weighting of incentives rewarded for the contemporaneous goal attainment increases. As discussed in Section 4.4.2, I use net income to measure the quality of the investment decisions made. Descriptive statistics presented in Table 18, Panel A show that the cumulative net income for periods 2-36 in the high contemporaneous weight condition (net income $=129,988)$ is higher than net income in the low $(114,247)$ or medium $(125,389)$ contemporaneous weight conditions (Hypothesis 2). ${ }^{49}$ This result is also shown graphically in Figure 10. The remainder of this section provides statistical analysis to examine this observation.

I begin my analysis by performing a repeated measures analysis of variance, where the within subjects, repeated measure is net income earned for each of the 35 periods and incentives is the between-subjects variable..$^{50,51}$ The dependent variable, net income, satisfies the assumptions required for parametric analysis: independence, normality and homogeneity (results not tabulated). However, the assumption of sphericity is not met. (Table 18, Panel B, $\mathrm{p}<0.01$, two tailed). In order to mitigate the bias the existence of sphericity introduces into

\footnotetext{
${ }^{49}$ As discussed in Section 4.3.1, in the low complexity setting, participants' investment decisions affect net income with a one period lag. Thus, the net income in period 1 does not reflect the quality of participant's investment decisions. Therefore period 1 net income has been excluded from the analyses of low complexity. ${ }^{50}$ I performed a second analysis (not tabulated) including experimental session (i.e., first or second administration) as an additional between subjects factor. Experiment session did not significantly the results of this analysis.

${ }^{51}$ Consistent with the hypothesis I am testing in this section, the analysis includes three incentive conditions: low, medium and high contemporaneous weightings. The performance of participants in the control condition is analyzed in Section 5.9.
} 
repeated measures analysis, I use the Huynh and Feldt correction factor in interpreting all repeated measures analysis in this section (Stevens 1996). ${ }^{52}$

The significance of period (Table 18, Panel C: $\mathrm{F}=9.32, \mathrm{p}<0.01$, two tailed) shows that over time, participants' investments become better, and they earn more net income per period. For example, in the first half of the simulation the cumulative net income earned (Net Income Periods 2-18 mean $=51,815$, not tabulated) is less than the net income earned in the last half of the simulation (Net Income Periods 19-36 mean $=69,362 \mathrm{p}<0.01$, one tailed, not tabulated).

Within-subjects, the interaction between period and incentives is not significant $(\mathrm{F}=$ $1.02, \mathrm{p}=0.44$, two tailed). Also, incentives do not significantly affect performance between subjects $(\mathrm{F}=1.30, \mathrm{p}=0.28$, two-tailed $)$.

Given the significant period effect, I proceed with my analysis by dividing the 35 periods into three stages: Periods 2-12, Periods $13-24$, and Periods 25 - 36. Doing so allows me to compare the performance of participants in each incentive condition at the beginning, the middle, and the end of the simulation. ${ }^{53}$ To further examine hypothesis two, I perform analyses of variance (where incentives is the independent variable and net income is the dependent variable) for each stage. The results show that the differences in net income between incentive contracts is not significant in the periods $2-12$ or $13-24$, but is significant in the final stage, periods 25-36. (Table 18, Panel D: Periods 2-12, $\mathrm{F}=0.35, \mathrm{p}=0.71$, two tailed; Periods $13-24, \mathrm{~F}=1.50, \mathrm{p}=0.23$, two tailed; Periods $25-35, \mathrm{~F}=3.61, \mathrm{p}=0.03$, two tailed). Although in most stages, participants in the high contemporaneous weighting

\footnotetext{
${ }^{52}$ The correction factor alters the degrees of freedom to adjust for the positive bias introduced into the test statistics due to the violation of the sphericity assumption (Stevens $1996 \mathrm{pg} 460$ ).

${ }^{53}$ The three stages identified also allow me to examine participant performance following the measurement of goal commitment at the end of periods 12 and 24 .
} 
incentive condition earned more net income than those in the low and medium

contemporaneous weighting incentive conditions (see raw data in Table 18, Panel A), the differences are only significant in the third stage (Periods 25-36). Planned contrasts of the net income differences between incentive conditions show that in Periods $25-36$, net income is significantly higher in the high contemporaneous weighting condition than in the low contemporaneous weighting condition (Table 18 , Panel E, $\mathrm{p}=0.01$, one tailed). ${ }^{54}$ Similar results are shown in the non-parametric tests presented in Table 18, Panels F and G.

Therefore, in the final stage of the simulation (Periods 25-36) I find evidence that is consistent with Hypothesis 2. The results show that, in a low complexity task, as the weighting of incentives rewarded for contemporaneous goal attainment increases, participants will make better investment decisions, and as a result earn higher levels of net income. This result is presented graphically in Figure 11.

\subsubsection{Test of Hypothesis 3}

In Hypothesis 3, I predict that individuals' commitment to the contemporaneous goal relative to the forward-looking goal will mediate the relationship between incentives and performance. To test this hypothesis, I perform mediation analysis (Baron and Kenny 1986). In this analysis the independent variable is the incentive contract, the mediating variable is the

\footnotetext{
${ }^{54}$ As discussed in chapter 4 given the lagged effect of investments on performance, the optimal strategy for participants would have been to not invest any Lira in the forward-looking metrics in the final period of the simulation. To test for this "end of game effect" I conducted a repeated measures analysis of variance, where the repeated measures was total investment in the last 12 periods of the simulation, and the between subjects variable was incentive condition. Within-subjects, period was not significant ( $\mathrm{p}=0.43$, two tailed, not tabulated) Therefore, this finding shows that participants do not significantly decrease their investments at the end of the experiment, and do not appear to engage in "end of game" strategies. Similar results are found in a separate analysis when only the last 2 periods of the simulation are included in the model (not tabulated).
} 
goal commitment difference score collected at the end of period 24, and the dependent variable is cumulative net income periods $25-36 .{ }^{55,56}$

To test mediation, I estimate three regressions, as specified by Baron and Kenny (1986). The three regressions are as follows:

1. Cumulative Net Income $=a_{0}+a_{1} *$ Incentive Contract Condition

2. Commitment Difference Score $=b_{0}+b_{1} *$ Incentive Contract Condition

3. Cumulative Net Income $=c_{0}+c_{1} *$ Incentive Contract Condition + $c_{2} *$ Commitment Difference Score

If $a_{1}, b_{1}$ and $c_{1}$ are significant, and the significance of $c_{1}$ is less than that of $a_{1}$ then the conditions set out in Baron and Kenny (1986) are satisfied, providing evidence that commitment mediates the relationship between incentive contract and investment decision accuracy.

As presented in Table 19, Panel B, the first regression provides evidence that incentive contract condition has a significant effect on investment decision accuracy $(\mathrm{p}<0.01$, one tailed). In the second regression, results show that the incentive contract assigned also significantly affects the commitment difference score in period $24(\mathrm{p}=0.01$, one tailed). The third regression does not satisfy the criteria of the Baron and Kenny (1986) mediation because the commitment difference score co-efficient is not significant. Therefore, I conclude that mediation does not exist, and the results are not consistent with Hypothesis $3{ }^{57}$ This result is presented graphically in Figure 12.

\footnotetext{
${ }^{55}$ As discussed in section 5.5.1, the goal commitment difference score is the difference between contemporaneous and forward-looking goal commitment.

${ }^{56}$ I select cumulative net income from this stage of the simulation because, as shown in section 5.6.1, this stage is the only stage where incentives significantly affected cumulative net income.

${ }^{57}$ I performed an additional analysis using the mean contemporaneous goal commitment instead of the commitment difference score. I also performed additional analyses where task characteristics, question order,
} 


\subsection{Investments in a High Complexity Task (Test of Hypotheses 4 and 6)}

Hypothesis 4 predicts that, in the high complexity conditions, participants who are rewarded for the attainment of both forward-looking and contemporaneous goals will make better investment decisions than those who are rewarded for the attainment of only contemporaneous goals. In comparing the performance of incentive conditions that reward both forward-looking and contemporaneous goal attainment, Hypothesis 6 predicts that individuals will make better investment decisions when incentives rewarded for contemporaneous goal attainment are weighted at least as heavily as the forward-looking goal incentives. To test both hypotheses, I compare the investment decisions of participants in the high, medium and low contemporaneous weight incentive contracts.

Similar to the analysis performed in section 5.6.1, I use net income to measure the quality of participants' investment decisions. ${ }^{58}$ The descriptive statistics for net income (Table 20, Panel A) show that, in most cases, participant's investments became better as the simulation progressed. Further inspection shows that the investments of participants in the medium contemporaneous weight condition earned, on average, more net income than those in the high contemporaneous condition (Hypothesis 4). In addition, it appears that those assigned a medium contemporaneous weighting contract earned more net income than those assigned a low contemporaneous weighting contract (Hypotheses 6). These results are depicted in Figure 13.

\footnotetext{
experimental session, and previous net income performance variables were included as additional variables. All analysis produced similar results to the mediation test reported in this section.

${ }^{58}$ Net income satisfies the assumptions required for parametric analysis: independence, normality and homogeneity (results not tabulated).
} 
I begin my analysis by performing a repeated measures analysis of variance, where the within subjects, repeated measure is the net income earned in each of the 33 periods and incentive condition is the between subjects variable (see Table 20, Panel C). ${ }^{59,60,61}$ The results of this analysis show that period is significant $(\mathrm{F}=4.01, \mathrm{p}<0.01$, two tailed $)$. As the simulation progressed, participants' made better investments, and earned more net income per period. For example, in the first half of the simulation the cumulative net income earned (Net Income Periods 4-19 mean $=41,131$, not tabulated) is less than the net income earned in the last half of the simulation (Net Income Periods 20-36 mean $=66.863, \mathrm{p}<0.01$, one tailed, not tabulated).

Given the significant effect of period on accuracy of investments, I proceed with my analysis by dividing the 33 periods into three stages: Periods $4-12$, Periods $13-24$, and Periods $25-36$. Analysis of variance where incentive condition is the independent variable and cumulative net income is the dependent variable shows that the effect of incentive contract assigned is significant in the final stage of the simulation (from Table 20, Panel D: Months $4-12, \mathrm{~F}=0.31, \mathrm{p}=0.73$, two-tailed, Months $13-24, \mathrm{~F}=1.36, \mathrm{p}=0.26$, two-tailed, Months $25-36, \mathrm{~F}=2.83, \mathrm{p}=0.06$, two-tailed).

To test Hypotheses 4 and 6, I use planned contrasts of the absolute mean differences between incentive groups (Table 20, Panel E). In Hypothesis 4, I predict that individuals who are rewarded for the attainment of both forward-looking and contemporaneous goals will

\footnotetext{
${ }^{59}$ As discussed in Section 4.3.1, in the high complexity setting, participants' investment decisions affect net income with a three period lag. Thus, the net income in periods 1-3 does not reflect the quality of participant's investment decisions. Therefore net income from these periods has been excluded from the analyses of high complexity.

${ }^{60}$ I performed a second analysis (not tabulated) including experimental session (i.e., first or second administration) as an additional between subjects factor. Experiment session does not significantly affect the results of this analysis.

${ }^{61}$ Consistent with the hypothesis I am testing in this section, the analysis includes three incentive conditions: low, medium and high contemporaneous weightings. The performance of participants in the control condition is analyzed in Section 5.9.
} 
make better investment decisions than those rewarded for the attainment of only

contemporaneous goals. To test this hypothesis, I compare the performance results of participants in the high contemporaneous conditions with those in the medium and low contemporaneous conditions. Results show that, in periods $25-36$, the difference in cumulative net income between the high and medium contemporaneous weighting incentive conditions is significant $(\mathrm{p}=0.07$, one-tailed), but the difference between the high and the low contemporaneous weighting incentive condition is not $(\mathrm{p}=0.50$, one-tailed $) .{ }^{62}$ Review of the descriptive statistics in Table 20, Panel A, show that participants in the medium condition earned significantly more net income (Periods $25-36$ mean net income $=60,982)$ than those in the high condition (Periods $25-36$ mean net income $=41,055$ ). However, participants in the high condition did not earn more than those in the low condition (Periods $25-36$ mean net income $=41,080$ ). Therefore, I find evidence to partially support Hypothesis 4. Similar results are found using non-parametric tests (Table 20, Panels F and G).

In Hypothesis 6, I predict that individuals will make better investment decisions when incentives rewarded for contemporaneous goal attainment are weighted more heavily than those for forward-looking goals. To test this hypothesis, I compare the performance results of participants in the low contemporaneous conditions with those in the medium contemporaneous conditions. ${ }^{63}$ Results show that, in periods $25-36$, the difference in cumulative net income between the low and medium contemporaneous weighting incentive

\footnotetext{
${ }^{62}$ Due to the three period lagged effect of investments on performance, the optimal strategy for participants would have been to not invest any Lira in the forward-looking metrics in the final three periods of the simulation. To test for this "end of game" effect I conducted a repeated measures analysis of variance, where the repeated measure was total investment in the last 12 periods of the simulation, and the between subjects variable was incentive condition. Within-subjects, period was not significant ( $\mathrm{p}=0.18$, one tailed, not tabulated) Therefore, this finding shows that participants do not significantly decrease their investments at the end of the experiment, and do not appear to engage in "end of game" strategies. (Similar results are found when only the last 4 periods of the simulation are included in the model (not tabulated).)

${ }^{63}$ Consistent with the hypothesis I am testing in this section, the analysis includes the two incentive conditions with non-zero weighting on the forward-looking measures (i.e., low and medium contemporaneous weights).
} 
conditions is significant (Table 20, Panel E: $p=0.05$, one-tailed). ${ }^{64}$ Review of the descriptive statistics in Table 20, Panel A, show that participants in the medium condition earned significantly more net income (Periods $25-35$ mean net income $=60,982$ ) than those in the low condition (net income $=41,080$ ). Therefore, I find evidence that is consistent with Hypothesis 6. The results discussed in this section are presented graphically in Figure 14.

\subsection{Mediation in a High Complexity Task (Tests of Hypotheses 5 and 7)}

In Hypotheses 5 and 7, I predict that individuals' commitment to the contemporaneous goal relative to the forward-looking goal will mediate the relationship between incentives and performance. In Hypothesis 5, I compare the relationship between incentives, goal commitment, and performance when only attainment of the contemporaneous goal is rewarded to conditions where both forward-looking and contemporaneous goals are rewarded. Similar to the analysis I performed in Section 5.6.2, to test this hypothesis I examine three different regression equations to test for mediation (Baron and Kenny 1986). In this analysis the independent variable is the incentive contract, the mediating variable is goal commitment, and the dependent variable is periods 25 - 36 cumulative net income. ${ }^{65}$ Given the results presented in Section 5.7 that incentive does not significantly affect performance in comparing the low and high contemporaneous weighting incentive contracts, I include only the medium and high incentive contracts in the mediation analysis.

As presented in Table 21, Panel A, the first regression model provides evidence that incentives have a significant effect on net income $(\mathrm{p}=0.02$, one tailed). In the second regression, results show that incentives do not significantly affect commitment $(\mathrm{p}=0.36$, one

\footnotetext{
${ }^{64}$ Similar results are found using non-parametric tests (Table 20, Panels F and G)

${ }^{65}$ As discussed in section 5.5.1, the goal commitment difference score is the difference between contemporaneous and forward-looking goal commitment.
} 
tailed). ${ }^{66}$ In the third regression, incentives $(p=0.02)$ significantly affects net income but commitment does not $(\mathrm{p}=0.16$, one tailed). Therefore the criteria of the Baron and Kenny (1986) mediation test are not satisfied, and I do not find evidence to support Hypothesis 5.

In Hypothesis 7, I posit that when incentives are rewarded for both contemporaneous and forward-looking goal attainment individuals' commitment to the contemporaneous goal, relative to that of the forward-looking goal, will mediate the relationship between incentives and performance. Similar to the analysis earlier in this section, to test this hypothesis I examine three different regression equations to test for mediation (Baron and Kenny 1986). In this analysis the independent variable is the incentive contract, the mediating variable is goal commitment, and the dependent variable is periods $25-36$ cumulative net income. ${ }^{67}$

Given my objective to examine incentive contracts that reward both forward-looking and contemporaneous goal attainment, I include only the low and medium incentive contracts in the mediation analysis.

As presented in Table 21, Panel B, the first regression provides evidence that incentive contract condition has a significant effect on net income $(p=0.01$, one tailed $)$. In the second regression, results show that the incentive contract assigned significantly affects the commitment difference score in period $24-36(\mathrm{p}<0.01$, one tailed $)$. In the third regression, both incentive contract $(p=0.06)$ and commitment difference score $(p=0.02$, one tailed $)$ are significant. The significance of the incentives in this regression is less than that in the first regression. Therefore the criteria of the Baron and Kenny (1986) mediation test are satisfied.

\footnotetext{
${ }^{66}$ This finding is consistent with the planned contrasts performed in section 5.5.1, where the comparison of commitment between the medium and high incentive conditions is not significant (Table 17, Panel G p $=0.50$ ).

${ }^{67}$ As discussed in section 5.5.1, the goal commitment difference score is the difference between contemporaneous and forward-looking goal commitment.
} 
To determine whether the indirect effect of the incentives on performance through the mediator variable (net income) is significant I use the Sobel test (Sobel 1982, Baron and Kenny 1986). The results show that the goal commitment has a marginally significant indirect effect on net income $(\mathrm{t}=1.39, \mathrm{p}$-value $=0.08$, one tailed, not tabulated $)$. Therefore, consistent with Hypothesis 7, I find evidence that goal commitment partially mediates the relationship between the incentives and net income. The results described in this section are presented graphically in Figure 15.

\subsection{Control Conditions}

Two control conditions are included in this study: a high complexity control condition and a low complexity control condition. Participants assigned to the control conditions were paid a flat rate of 600 Lira a month but were assigned the same goals. To examine how control group participants perform in comparison with the other participants, I compare the net income and task complexity across conditions in both the high and low complexity conditions. ${ }^{68}$

In the low complexity condition, the average net income (periods $2-36$ ) in the control condition is 125,786 (Table 18, Panel A). Net income in the control condition is less than that in the high $(129,988)$ condition, comparable to that in the medium condition $(125,389)$ and more than that in the low condition $(114,247)$. However, none of the differences involving the control condition are significant (Table 22, Panel A: high $\mathrm{p}<0.99$, two tailed, medium $\mathrm{p}<$ 0.99 , two tailed, low $\mathrm{p}<0.99$, two tailed).

\footnotetext{
${ }^{68}$ There were two differences between the operalization of the control and non-control conditions that may have made the simulation task in the control condition less difficult than the other conditions. First, the control condition participants were not asked the goal commitment questions at the end of periods 12 and 24. Second, control participants were paid a flat rate for the task, and their wages were not dependent upon performance.
} 
Participants in the low complexity control (Table 10, Panel A: -0.39) condition found the task to be less complex than those in the low (mean complexity score $=0.11$ ), medium (0.17) or high (0.42) conditions. The differences between the control and medium and high conditions are significant (Table 22, Panel B: high $\mathrm{p}=0.13$; medium $\mathrm{p}=0.07$; low $\mathrm{p}=0.01$ one tailed, adjusted).

In the high complexity condition, review of the descriptive statistics show that, the net income earned by the participants in the control (Table 20, Panel A: 133,301) condition is similar to those in the medium $(135,657)$ contemporaneous weight condition (Table 22, Panel C: $p<0.99$, two tailed). Participants in the low $(96,220)$ and high $(95,840)$ contemporaneous weighting condition earned less net income than those in the control condition. However, the differences between conditions are not significant (Table 22, Panel C: low $\mathrm{p}=0.51$, twotailed, adjusted; high $\mathrm{p}=0.84$, two-tailed, adjusted).

Participants in the high complexity control (Table 10, Panel A: 0.50) condition found the task to be less complex than those in the low (0.58) or high (1.04) conditions. However, only the difference between the high and control conditions is significant (Table 22, Panel B: high $\mathrm{p}=0.07 ;$ medium $\mathrm{p}=0.50 ;$ low $\mathrm{p}=0.50$, one tailed, adjusted).

\subsection{Summary}

This chapter provides results obtained from the tests of hypotheses. Generally, the presence of forward-looking and contemporaneous goal based incentives is shown to affect manager performance. As the weighting of contemporaneous goal attainment increases in the incentive contract, participants' level of commitment to the contemporaneous goal, relative to the forward-looking goal increases. (Hypothesis 1 is supported.) Participants in the high 
contemporaneous weighting condition performed significantly better than those in the low contemporaneous weighting condition. (Hypothesis 2 is supported.) In a low complexity task, contemporaneous goal commitment, relative to forward-looking commitment, does not mediate the relationship between incentives and performance. (Hypothesis 3 is not supported.)

In a high complexity setting, participants made significantly better investment decisions when assigned an incentive contract with medium weighting on contemporaneous measures, than when a high weighting on contemporaneous measures was assigned. (Hypothesis 4 is partially supported.) Participants assigned an incentive contract with a medium contemporaneous incentive weight incentive contract also made better investment decisions than those assigned a low contemporaneous incentive weight incentive contract. (Hypothesis 6 is supported.) In a high complexity setting, the relationship between incentive contracts and performance is not mediated by goal commitment when comparing incentives with medium and high contemporaneous incentive weights, but is mediated by goal commitment when comparing incentives with a low and medium contemporaneous incentive weight. (Hypothesis 5 is not supported, Hypotheses 7 is supported.) A summary of these results is presented in Table 23 . 


\section{CHAPTER 6: CONCLUSION}

\subsection{Introduction}

In this chapter I discuss the hypotheses testing in Section 6.2. The control condition results are discussed in Section 6.3. In Section 6.4, I identify limitations of this study, and opportunities for future research. Finally, I conclude in Section 6.5.

\subsection{Discussion of Hypotheses Testing Results}

The results presented in Chapter 5 show that five of the seven hypotheses tested are fully or partially supported. (See Table 23 for a summary.) Collectively these findings provide evidence that in a long-term horizon setting, differences in the incentive contracts weights placed on forward-looking and contemporaneous measures influence the quality of resource allocation decisions. These findings also provide evidence that task complexity influences the expected effect of various incentive weights on management decisions.

In both high and low complexity tasks, as the weighting of incentives rewarded for contemporaneous goal attainment increases, individuals' commitment to the contemporaneous goal, relative to that of the forward-looking goal increases (Hypothesis 1). Consistent with

prior research in single goal settings (Hollenbeck and Klein 1987; Locke et al. 1988), this result provides evidence that in settings where multiple goals are assigned, incentives rewarded for goal attainment can be used by organizations to increase or decrease an individual's commitment to a goal, relative to the other goals assigned.

Consistent with my prediction, in a low complexity task, as the weighting of incentives rewarded for contemporaneous goal attainment increases, individuals make better investment decisions, and as a result perform better on contemporaneous measures 
(Hypothesis 2). This finding provides evidence that when individuals are rewarded more heavily for forward-looking goal attainment, they may make decisions that increase their own personal wealth, even though such decisions do not improve the long-term performance of the organization. As noted in chapter 2, such behaviour is undesirable for organizations. To encourage individuals to make decisions congruent with maximizing the long-term performance of the organization, they should be rewarded more heavily for contemporaneous goal attainment than forward-looking goal attainment. This finding applies to incentive contracts where only the contemporaneous goal is rewarded, as well as incentive contracts where both contemporaneous and forward-looking goals are rewarded. As discussed in Chapter 2, some organizations use incentive contracts that reward employees for both forward-looking and contemporaneous performance (Ittner et al. 1997; Kaplan and Norton 2006). To encourage employees to make decisions that are congruent with the long-term objectives of the organization, my findings suggest that managers at these organizations should ensure that, when employees are performing tasks of low complexity, the forwardlooking measures are rewarded less heavily than the contemporaneous measure.

Contrary to my expectation, I do not find evidence that, in a setting of low complexity, relative levels of contemporaneous goal commitment mediates the relationship between incentives and performance (Hypothesis 3). Instead, when incentives and goal commitment are included in the same regression model, incentives have a significant effect of performance, but commitment does not. In low complexity tasks this finding may be attributed to the characteristics of the task. In tasks of low complexity, the strategies required to attain a contemporaneous goal are often easily identified and implemented by individuals. When individuals quickly learn successful strategies the decision facilitating benefits of goals, 
and participants' commitment to them, are less consequential than in high complexity tasks (Wood et al. 1990, Winters and Latham 1996). Thus, in a low complexity task my results suggest that participants' level of contemporaneous goal commitment relative to forwardlooking goal commitment is less influential in selecting task strategies than the incentive contract assigned.

Consistent with my expectations, in a high complexity setting, individuals make better investment decisions when incentives rewarded for contemporaneous goal attainment are weighted more heavily than those for forward-looking goal incentives (Hypothesis 6). However, unlike the results found in the low complexity setting, in a high complexity setting individuals who are rewarded for the attainment of both forward-looking and contemporaneous goals make better investment decisions than those who are rewarded only for contemporaneous performance (Hypothesis 4). As noted in Chapter 2, some researchers argue that, when an employee has a long-term horizon, the most effective contracts should only reward contemporaneous measures (Dikolli 2002, Dutta and Reichelstein 2003). In a task of high complexity, this study provides evidence contrary to this claim. I find evidence that incentives on both forward-looking and contemporaneous measures are necessary for individuals to develop task related strategies that are consistent with the organization's longterm objectives.

In a setting of high complexity, when comparing low and high contemporaneous weight incentive contracts (Hypothesis 7) contemporaneous goal commitment, relative to forward-looking goal commitment, mediates the relationship between incentives and performance. However, when comparing medium and high contemporaneous weight incentive contracts (Hypothesis 5) goal commitment does not mediate the relationship 
between incentives and performance. The insignificant effect of incentives on goal commitment is the cause of this unexpected result. Contrary to my expectation, as the weighting of incentives rewarded for contemporaneous goal attainment increases, individuals' relative levels of contemporaneous goal commitment did not increase. ${ }^{69}$ Review of the goal commitment scores suggests that participants in the medium contemporaneous weighting condition increase their commitment to contemporaneous goals, relative to forward-looking goals between periods 12 and 24 (Table 17, Panel B: difference score $=-0.54$; difference score $=0.35, \mathrm{p}<0.01$, not tabulated), while those in the high contemporaneous weighting condition do not significantly change their goal commitment levels (period $12=0.32$; period $24=0.25, \mathrm{p}=0.74$, not tabulated). This result may be explained by the participants' success in identifying successful allocation strategies by period 24 in the simulation. By period 24, participants in the medium contemporaneous condition are likely to have learned that forward-looking measures are designed to facilitate their attainment of the contemporaneous measure and that contemporaneous measure goal attainment is the best strategy to increase their earnings. As a result, their commitment to the contemporaneous goal, relative to their commitment to the forward-looking goals significantly increases. At the same point in the simulation, participants in the high contemporaneous weighting condition may not have identified successful allocation strategies, and may not recognize the importance of the forward-looking measures in increasing their own wealth. As a result their commitment to the contemporaneous goal relative to their commitment to the forward-looking goals is not significantly affected.

\footnotetext{
${ }^{69}$ While a significant difference between incentive conditions exists at period 12 (Table 17, Panel G, $\mathrm{p}=0.09$ ), the difference between conditions at period 24 is insignificant $(\mathrm{p}=0.50)$.
} 
Overall, the results of this study show that incentive contracts significantly affect contemporaneous goal commitment, relative to that of the forward-looking goal (Hypothesis 1) and performance (Hypotheses 2, 4 and 6). Contrary to my expectation, I do not find evidence that goal commitment mediated the relationship between incentive contracts and performance in a low complexity setting (Hypothesis 3). However, in a high complexity setting, I find some evidence of the mediating relationship of goal commitment on performance in the high complexity conditions (Hypothesis 7).

\subsection{Discussion of the Control Conditions}

As discussed in Chapter 2, previous research finds evidence the assignment of goals, without incentives rewarded for goal attainment often results in worse performance than when goal attainment is rewarded with incentives. However, in my study, participants in the control condition (i.e., those assigned goals, but not rewarded incentives for their attainment) performed just as well as individuals in conditions where goal attainment was rewarded. This observation is not consistent with the previous literature. A possible explanation for this is that the design of the control condition in my study may have made the simulation task in the control condition less difficult than the other conditions. There were two differences between the operalization of the control and non-control conditions that may have influenced results. First, the control condition participants were not asked the goal commitment questions at the end of periods 12 and 24. Instead control participants completed the 36-period simulation uninterrupted. The task interruptions in the non-control conditions may have increased the overall difficulty of the task. Second, control participants were paid a flat rate of $\$ 21.60$ to complete the task. Their earnings were significantly higher than the average earnings of the 
other participants $(\$ 18.70, \mathrm{p}<0.01$, one tailed). Because the control participants were paid significantly more than the other participants they may have been more committed to the overall task, exerted more task related effort, and as a result, performed better than the other participants (Locke and Latham 2002).

\subsection{Limitations and Opportunities for Future Research}

This study has various limitations that provide opportunities for future research. First, the results of this study may not be generalizable to individuals with different backgrounds. ${ }^{70}$ Future research might examine the impact of goals and incentives on a more diverse sample. Second, the results of this study may be sensitive to the goal structures I have created. Future research might examine settings with a different goal sets. For example, a setting where multiple contemporaneous and forward-looking goals could be examined in future research. Third, in this study I examine three different incentive contract weightings. Future research might examine settings with different compensation structures, where different weightings are used for high, medium, and low contemporaneous weighting incentive contracts. Fourth, the experimental task is a simplified version of actual decision-making environments in which managers operate. For example, individuals in my experiment made investment decisions in an environment where forward-looking measures had a direct, non-linear relationship to contemporaneous results and all forward-looking measures had been identified. Future research might examine how inaccurate and incomplete forward-looking information impact performance, and how different relationships between forward-looking and contemporaneous measures affect performance. Fifth, I examine a setting where all participants have a long-

\footnotetext{
${ }^{70}$ As noted in Section 5.2, 58\% of participants were in an accounting and $29 \%$ of participants were in a mathematics program (Table 7).
} 
term employment horizon. Future research might examine determinates of a long-term employee horizon, and the effect on performance when the employee horizon is uncertain. Sixth, the results of this study may be sensitive to my experimental design. In this study, I reward participants for exceeding their assigned goals. This feature of the design provides an opportunity for employees to shift their effort amongst measures, and act in a manner that increases their own personal wealth while negatively affecting organizational performance. Future research should examine the effect of other effort shifting opportunities on performance. Sixth, I examine a setting where individuals are rewarded for goal attainment. My results may not generalize to other types of incentive contracts. Future research should examine the effect of difference incentive contracts, such as a piece rate incentive scheme, on performance. Finally, I examine a setting where the organizational objective is to maximize its net income. This setting may not apply to all organizations. Future research might examine settings where the organizational objective is to maximize other contemporaneous measures.

\subsection{Conclusions}

I believe this study will make a valuable contribution to the incentive contracting literature. The existing literature has not examined how various weightings of forwardlooking incentives affect the quality of individuals' decision making. Furthermore, the existing literature does not examine the impact of task complexity on the relationship between incentives and performance. This research contributes to the literature by demonstrating that in both low and high task complexity settings, incentive contracts that weight forward-looking incentives too heavily cause forward-looking information to become decision influencing 
which can negatively impact performance. However, this research also provides evidence that, unlike in low complexity tasks, in tasks of high complexity incentive contracts that provide some financial rewards for forward-looking goal attainment (in addition to contemporaneous goals) lead to better performance than incentive contracts that do not reward forward-looking goal attainment. 


\section{REFERENCES}

Atkins, P.W.B., R. E. Wood, and P. J. Rutgers. 2002. "The effects of feedback format on dynamic decision making." Organizational Behavior and Human Decision Processes 88: 587-604.

Aseff, J. G. and M. S. Snatos. 2005. "Stock Options and Managerial Optimal Contracts." Economic Theory 26: 813-837.

Bailey, W, G. Hecht, and K. L. Towry. 2006. "Dividing the Pie: Do Managers Fully Incorporate Non-Contracted Information into Full and Partial Discretionary Bonus Allocations?" Unpublished Working Paper, University of South Carolina/Emory University.

Balzer, W. K., Doherty, M. E., \& O'Connor Jr, R. 1989. "Effects of cognitive feedback on performance.” Psychological Bulletin, 106(3), 410-433.

Bandura, A. and K. M. Simon. 1977. "The Role of Proximal Intentions in Self-Regulation of Refractory Behavior." Cognitive Therapy and Research 1(3): 177-193.

Bandura, A. and R. Wood. 1989. "Effect of perceived controllability and performance standards on self-regulation of complex decision-making." Journal of Personality and Social Psychology 56: 805-814

Banker, R. D., Hsihui C., and M. J. Pizzini. 2004. "The Balanced Scorecard: Judgmental Effects of Performance Measures Linked to Strategy." The Accounting Review 79 (1): 123.

Banker, R. D., G. Potter, and D. Srinivasan. 2000. "An Empirical Investigation of an Incentive Plan that Includes Nonfinancial Performance Measures." The Accounting Review 75(1): 65-92.

Baron, R. M., \& Kenny, D. A. (1986). “The moderator-mediator variable distinction in social psychological research: Conceptual, strategic, and statistical considerations." Journal of Personality and Social Psychology, 51: 1173-1182.

Berry, L. 2001. Improve Service by Acting Small." Managing Service Quality 11(2): 75-79.

Bonner, S. E. 1994. "A Model of the Effects of Audit Task Complexity." Accounting, Organizations and Society 19(3): 213-234. 
Bonner, S. E., R. Hastie, G. B. Sprinkle, and S. Young. 2000. "A review of the effects of financial incentives on performance in laboratory tasks: implications for management accounting." Journal of Management Accounting: 19-64.

Bonner, S. E. and G. B. Sprinkle. 2002. "The Effects of Monetary Incentives on Effort and Task Performance: Theories, Evidence, and a Framework for Research." Accounting, Organizations and Society 27: 303-345.

Brehmer, B. 1996. "Man as a Stabiliser of Systems: From Static Snapshots of Judgment Processes to Dynamic Decision Making” Thinking and Reasoning 2(2-3): 225-238.

Bryant, L., D. A. Jones, and S. K. Widener. 2004. "Managing Value Creation within the Firm: An Examination of Multiple Performance Measures." Journal of Management Accounting Research 16: 107-131.

Bukless, F.A., and S.P. Ravenscroft. 2000. "Contrast Coding: A Refinement of ANOVA in Behavioral Analysis." The Accounting Review, 65 (4): 933-945

Camerer, C. F., L. Babcock, G. Lowenstein, and R. Thaler. 1997. "Labor Supply of New York City Cab Drivers: One Day at a Time." The Quarterly Journal of Economics 112(2): 407441.

Campbell, D. J. 1988. "Task Complexity: A Review and Analysis." The Academy of Management Review 13(1): 40-52

Cardineales E. 2008. "The interplay between cost accounting knowledge and presentation formats in cost-based decision-making" Accounting, Organizations and Society 33(6): 582-602

Chesney, A. A. and E. A. Locke. 1991. "Relationships among Goal Difficulty, Business Strategies, and Performance on a Complex Management Simulation Task." The Academy of Management Review 34(2): 400-424.

Cohen, J. "A Power Primer." Psychological Bulletin 112, no. 1 (1992): 155-159.

Datar, S.M., Feltham, G.A., Hughes, J.S., 1991. The role of audits and audit quality in valuing new issues. Journal of Accounting and Economics 14, 3-49.

Deloitte Touche Tohmatsu. 2007. In the Dark II: What many boards and executives still don't know about the health of their businesses. New York, NY 
DeShon, R. P. and R. A. Alexander. 1996. "Goal Setting Effects on Implicit and Explicit Learning of Complex Tasks." Organizational Behavior and Human Decision Processes 65(1): 18-36.

Demski, J. S., and G. A. Feltham. 1976. Cost Determination: A Conceptual Approach. First edition. Iowa State University Press, Ames, IA

Dess, G.G., and D.W. Beard 1984 "Dimensions of Organizational Task Environments" Administrative Science Quarterly, 29(1): 52-73

Dhaliwal, J.S., and I. Benbasat. 1996. "The Use and Effects of Knowledge-Based System Explanations: Theoretical Foundations and a Framework for Empirical Evaluation" Information Systems Research 7(3): 342-362

Diehl, E., and J.D. Sterman, 1995. "Effects of Feedback Complexity on Dynamic Decision Making." Organizational Behavior and Human Decision Processes 62(2): 198-215.

Diekhoff, G. 1992 "Statistics for the social and behavioral sciences: univartiate, bivariate, multivariate" Brown Publishers

Dikolli, S. S. 2001. "Agent Employment Horizons and Contracting Demand for ForwardLooking Performance Measures." Journal of Accounting Research 39(3): 481-494.

Drake, A.R., S. F. Haka, and S.P. Ravenscroft. 1999. "Cost System and Incentive Structure Effects on Innovation, Efficiency and Profitability in Teams." The Accounting Review, 74 (3): $323-345$

Dutta, S. and S. Reichelstein. 2003. "Leading Indicator Variables, Performance Measurement, and Long-term Versus Short term Contracts." Journal of Accounting Research 41(5): 837-866.

Earley, P. C., T. Connolly, and G. Ekegren. 1989. "Goals, Strategy Development, and Task Performance: Some Limits on the Efficacy of Goal Setting." Journal of Applied Psychology 74(1): 24-33.

Earley, P. C. and T. R. Lituchy. 1991 "Delineating Goal and Efficacy Effects: A Test of Three Models." Journal of Applied Psychology 76(1): 81-98.

Earley, P. C., C. E. Shalley, and Gregory B. Northcraft. 1992. "I Think I can, I Think I can...Processing Time and Strategy Effects of Goal Acceptance/Rejection Decisions." Organizational Behavior and Human Decision Processes 53: 1-13. 
Farrell, A.M, Kadous, K., Towry, K. L. 2008. Contracting on contemporaneous versus forward-looking measures: an experimental investigation. Contemporary Accounting Research 25 (3): 773-802

Feltham G. and J Xie. Fairness and incentives in principal-agent model. The Accounting Review 69(3): 429-453

Fessler, N. J. 2003. "Experimental Evidence on the Links among Monetary Incentives, Task Attractiveness and Task Performance." Journal of Management Accounting Research 15: 161-176.

Fishbach, A., R. Dhar, and Ying Zhang. 2006. "Subgoals as Substitutes Or Complements: The Role of Goal Accessibility." Journal of Personality and Social Psychology 91(2): 232242.

Fisher, J. G., L. A. Maines, S.A. Peffer, and G. B. Sprinkle. 2005. "An Experimental Investigation of Employer Discretion in Employee Performance Evaluation and Compensation." The Accounting Review 80 (2): 563-583.

Fisher, J. G., S. A. P., and G. B. Sprinkle. 2003. "Budget-Based Contracts, Budget Levels, and Group Productivity." Journal of Management Accounting Research 15: 51-74.

Gilliland S.W., and R.S. Landis. 1992. "Quality and quantity goals in a complex decision task : strategies and outcomes" Journal of applied psychology 77(5): 672-681

Goll, I., A. M. A. Rasheed. 1997. "Rational Decision-Making and Firm Performance: The Moderating Role of Environment.” Strategic Management Journal, 18(7): 583-591

Frederickson, J. R., S. A. Peffer, and J. Pratt. 1999. "Performance Evaluation Judgments: Effects of Prior Experience Under Different Performance Evaluation Schemes and Feedback Frequencies." Journal of Accounting Research 37(1): 151-165.

Hall, B. J. and K. J. Murphy. 2003."The Trouble with Stock Options." The Journal of Economic Perspectives 17(3): 49-70.

Hannan, R. L. 2005. "The Combined Effect of Wages and Firm Profit on Employee Effort." The Accounting Review 80(1): 167-188.

Heath, C., R. P. Larrick, and G. Wu. 1999. "Goals as Reference Points." Cognitive Psychology 38: 79-109. 
Hendricks, K., L. Menor, and C. Wiedman. 2004. "Adoption of the Balanced Scorecard: A Contingency Variables Analysis." Unpublished Working Paper, University of Western Ontario and University of Waterloo

Hollenbeck, J. R. and H. J. Klein. 1987. "Goal Commitment and the Goal-Setting Process: Problems, Prospects and Proposals for Future Research." Journal of Applied Psychology 72(2): 212-220.

Hollenbeck, J. R., C. R. Williams, and H. J. Klein. 1989. "An Empirical Examination of the Antecedents to Commitment to Difficult Goals." Journal of Applied Psychology 74(1): $18-23$.

Hsee, C.K., F. Yu, J. Zhang and Y. Zhang. 2003. "Medium Maximization." Journal of Consumer Research 30: 1-14.

Indjejikian, R .J. and D. Nanda. 2002. "Executive Target Bonuses and what they Imply about Performance Standards." The Accounting Review 77(4): 793-819.

Ittner, C. D. and D. F. Larcker. 1998. "Are Non-financial Measures Leading Measures of Financial Performance? An Analysis of Customer Satisfaction." Journal of Accounting Research 36: 1-35.

Ittner, C. D., D. F. Larker, and M. V. Rajan. 1997. The Choice of Performance Measures in Annual Bonus Contracts." The Accounting Review 72(2): 231-255.

Ittner, C. D., D. F Larker, and M. W. Meyer. 2003. "Subjectivity and the Weighting of Performance Measures: Evidence from a Balanced Scorecard." The Accounting Review 78(3): 725-758.

Ittner, C. D., W.N. Lanen, and D. F Larker. 2002. "The association between activity-based costing and manufacturing performance." Journal of Accounting Research 40(3): 711726.

Kanfer, R.. P.L. Ackerman, T.C. Murtha, B. Dugdale, and L. Nelson. 1994. "Goal setting, conditions of practice, and task performance: a resource allocation perspective" Journal of Applied Psychology 79(6): 826-835.

Kaplan, R. S. and D. P. Norton. 2006. Alignment: Using the Balanced Scorecard to Create Corporate Synergies. Boston, Massachusetts: Harvard Business School Press. 
_ 1996. "Using the Balanced Scorecard as a Strategic Management System." Harvard Business Review January-February: 75-85.

_ 1993. "Putting the Balanced Scorecard to Work." Harvard Business Review September-October: 1-15.

Kelly, K. 2007. "Feedback and Incentives on Non-Financial Value Drivers: Effects on Managerial Decision Making." Contemporary Accounting Research 24(2): 523-556

_ 2009. "Rewarding Multiple Leading Measures of Financial Performance: Does Accuracy of Relative Incentive Weights Matter?" Unpublished Working Paper, University of Waterloo.

Kessler, L. and R. H. Ashton. 1981. Feedback and prediction achievement in financial analysis. Journal of Accounting Research (Spring): 146-162.

Klein, H.J. 1991. "Further evidence on the relationship between goal setting and expectancy theories." Organizational Behavior and Human Decision Processes, 49: 230-257

Klein H.J., and P.M. Wright 1994. "Antecedents of Goal Commitment: An Empirical Examination of Personal and Situational Factors" Journal of Applied Psychology 24(2):95-114

Klein, H. J., M. J. Wesson, J. R. Hollenbeck, and B. J. Alge. 1999. "Goal Commitment and the Goal-Setting Process: Conceptual Clarification and Empirical Synthesis." Journal of Applied Psychology 84(6): 885-896.

Klein, H. J., M. J. Wesson, J. R. Hollenbeck, P. M. Wright, and R. P. DeShon. 2001. "The Assessment of Goal Commitment: A Measurement Model Meta Analysis." Organizational Behavior and Human Decision Processes 85(1): 32-55.

Krishnan R., J.L. Luft and M.D. Shields. 2005. "Effects of accounting-method choices on subjective performance-measure weighting decisions: experimental evidence on precision and error covariance.” The Accounting Review 80(4): 1163-1192

Latham, G. P. and G. H. Seijts. 1999. "The Effects of Proximal and Distal Goals on Performance on a Moderately Complex Task." Journal of Organizational Behavior 20: 421-429.

Latham, G. P., D. Winters, and E. A. Locke. 1994. "Cognitive and Motivational Effects of Participation: A Mediator Study." Journal of Organizational Behavior 25(1): 49-63. 
Leung, P.W. and K.T. Trotman. 2005. "The effects of feedback type on auditor judgment performance for configural and non-configural tasks" Accounting, Organizations and Society 30(6): 537-553

Libby, R. and Lipe, M. G. "Incentive Effects and the Cognitive Processes Involved in Accounting Judgments." Journal of Accounting Research (Autumn 1992): 249-273.

Libby, T. 2001. "Referent Cognitions and Budgetary Fairness: A Research Note." Journal of Management Accounting Research 13(1): 91-105.

Libby, T., S. E. Salterio, and A. Webb. 2004. "The Balanced Scorecard: The Effects of Assurance and Process Accountability on Managerial Judgment." The Accounting Review 79(7): 1075-1094.

Lipe, M. G. and S. E. Salterio. 2000. "The Balanced Scorecard: Judgmental Effects of Common and Unique Performance Measures." The Accounting Review 75 (3): 283-298.

Locke, E. A., Latham, G. P., \& Erez, M. 1988. The determinants of goal commitment. Academy of Management Review, 13, 23-39.

Locke, E. A., Frederick, E., Lee, C. \& Bobko, P. 1984. The effects of self-efficacy, goals and task strategies on task performance. Journal of Applied Psychology, 69, 241-251.

Locke, E.A. and G.P. Latham. 2002. "Building a Practically Useful Theory of Goal Setting and Task Motivation: A 35-Year Odyssey." American Psychologist 57: 705-717.

Locke, E.A., G.P. Latham, and Miriam Erez. 1998. "The Determinants of Goal Commitment." Academy of Management Review 13(1): 23-39.

Locke, E.A., K.N. Shaw, L.M. Saari and G.P. Latham. 1981. "Goal setting and task performance: 1969-1980.” Psychological Bulletin 90(1): 125-152

Malina, M.A. and F.H. Selto. 2001. "Communicating and Controlling Strategy: An Empirical Study of the Effectiveness Of the Balanced Scorecard." Journal of Management Accounting Research 13: 47-90.

Meyer, M.W. Rethinking Performance Measurement. First ed. Cambridge: University Press, 2002. 
Miller, D. 1988. "Relating Porter's Business Strategies to Environment and Structure: Analysis and Performance Implications." The Academy of Management Journal, 31(2): 280-308

Morgan, M. 1985. "Self-Monitoring of Attained Subgoals in Private Study." Journal of Educational Psychology 77(6): 623-630.

Murphy, K.J. and P. Oyer. 2003. "Discretion in Executive Incentive Contracts." unpublished working paper, University of Southern California/Stanford University.

Nagar, V. 2002. "Delegation and Incentive Compensation." The Accounting Review 77(2): 379-395.

O'Connell, P. 2006. "Taking the Measure of Mood." Harvard Business Review March

Pelham, B.W. and E. Neter. 1995. "The Effect of Motivation of Judgment Depends on the Difficulty of the Judgment." Journal of Personality and Social Psychology 68(4): 581594.

Ross, J.A. 2007. "Human Capital Readiness at Global Restaurant Franchise Autogrill." Balanced Scorecard Report January - February

Schulz, A., A. Webb., and Scott J. 2008. "The Performance Effects of Two Approaches to Setting Stretch Goals." University of Melbourne/University of Waterloo.

Seijts, G.H. and G.P. Latham. 2001. "The effect of distal learning, outcome, and proximal goals on a moderately complex task." Journal of Organizational Behavior 22(3): 291 307

Seijts, G.H. and G.P. Latham. 2005. "Learning Versus Performance Goals: When should each be used?" Academy of Management Executive 19 (1): 124-131.

Seijts, G.H., G.P. Latham, K.Tasa, and B.W. Latham. 2004. "Goal Setting and Goal Orientation: An Integration of Different Yet Related Literatures." Academy of Management Journal 47 (2): 227-239.

Sengupta, K., T.K. Abdel-Hamid. 1993. "Alternative Conceptions of Feedback in Dynamic Decision Environments: An Experimental Investigation.” Management Science 39(4): 411-428. 
Sliwka, D. 2002. "On the use of Nonfinancial Performance Measures in Management Compensation." Journal of Economics \& Management Strategy 11(3): 487-511.

Smith, M.J. 2002. "Gaming Nonfinancial Performance Measures." Journal of Management Accounting Research 14: 119-133.

Sobel, M. E. 1982. "Asymptotic confidence intervals for indirect effects in structural equation models.” In S Leinhardt (Ed.), Sociological Methodology 1982 (pp. 290-312). San Francisco: Jossey-Bass

Sprinkle, G.B. 2000. "The Effect of Incentive Contracts on Learning and Performance." The Accounting Review 75(3): 299-326.

Sprinkle, G.B. 2003. Perspectives on experimental research in managerial accounting. Accounting, Organizations and Society, 28, 287-318

Stevens, J. 1996. Applied Multivariate Statistics for the Social Sciences. Mahwah, MJ: Erlbaum

Sterman, J.D. 1989. "Modeling Managerial Behavior: Misperceptions of Feedback in a Dynamic Decision Making Experiment.” Management Science 35(3): 321-339.

Stajkovic, A.D. and F. Luthans. 1998. "Self- Efficacy and Work-Related Performance: A Meta-Analysis." Psychological Bulletin 124(2): 240-261.

Sternman, J.D. 1989, "Modeling managerial behavior: misperceptions of feedback in a dynamic decision making experiment." Management Science 35(3): 321-339

Towry, K.L. 2003. "Control in a Teamwork Environment - the Impact of Social Ties on the Effectiveness of Mutual Monitoring Contracts." The Accounting Review 78(4): 10691095.

Ullrich, M. and B.M. Tuttle. 2004. "The Effects of Comprehensive Information Reporting Systems and Economic Incentives on Managers' Time-Planning Decisions." Behavioral Research in Accounting 16: 89-105.

Webb, A. 2004. "Managers' Commitment to the Goals Contained in a Strategic Performance Measurement System." Contemporary Accounting Research 21(4): 925-958.

Winters, D. and G.P. Latham. 1996. "The Effect of Learning Versus Outcome Goals on a Simple Versus a Complex Task." Group and Organization Management 21(2): 236-250. 
Wood, R.E.. 1986. "Task Complexity: Definition of the Construct." Organizational Behavior and Human Decision Processes 37: 60-82.

Wood, R.E., A. J. Mento, and E. A. Locke. 1987. "Task Complexity as a Moderator of Goal Effects: A Meta-Analysis." Journal of Applied Psychology 72(3): 416-425.

Wood, R.E., P. Atkins, and C. Tabernero. 2000. "Self-Efficacy and Strategy on Complex Tasks." Applied Psychology: An International Review 49(3): 430-446.

Wood, R.E. and A. Bandura. 1989. "Effect of Perceived Controllability and Performance Standards on Self-Regulation of Complex Decision Making." Journal of Personality and Social Psychology 56(5): 805-814.

Wood, R.E, A. Bandura, and Trevor Bailey. 1990. "Mechanisms Governing Organizational Performance in Complex Decision-Making Environments." Organizational Behavior and Human Decision Processes 46: 181-201.

Wright P.M., and K.M. Kacmar 1995. "Mediating roles of self-set goals, goal commitment, self-efficacy, and attractiveness in the incentive-performance relation." Human Performance 8: 263-296 


\section{FIGURES}

Figure 1

Relationship between Forward-Looking and Contemporaneous Goals

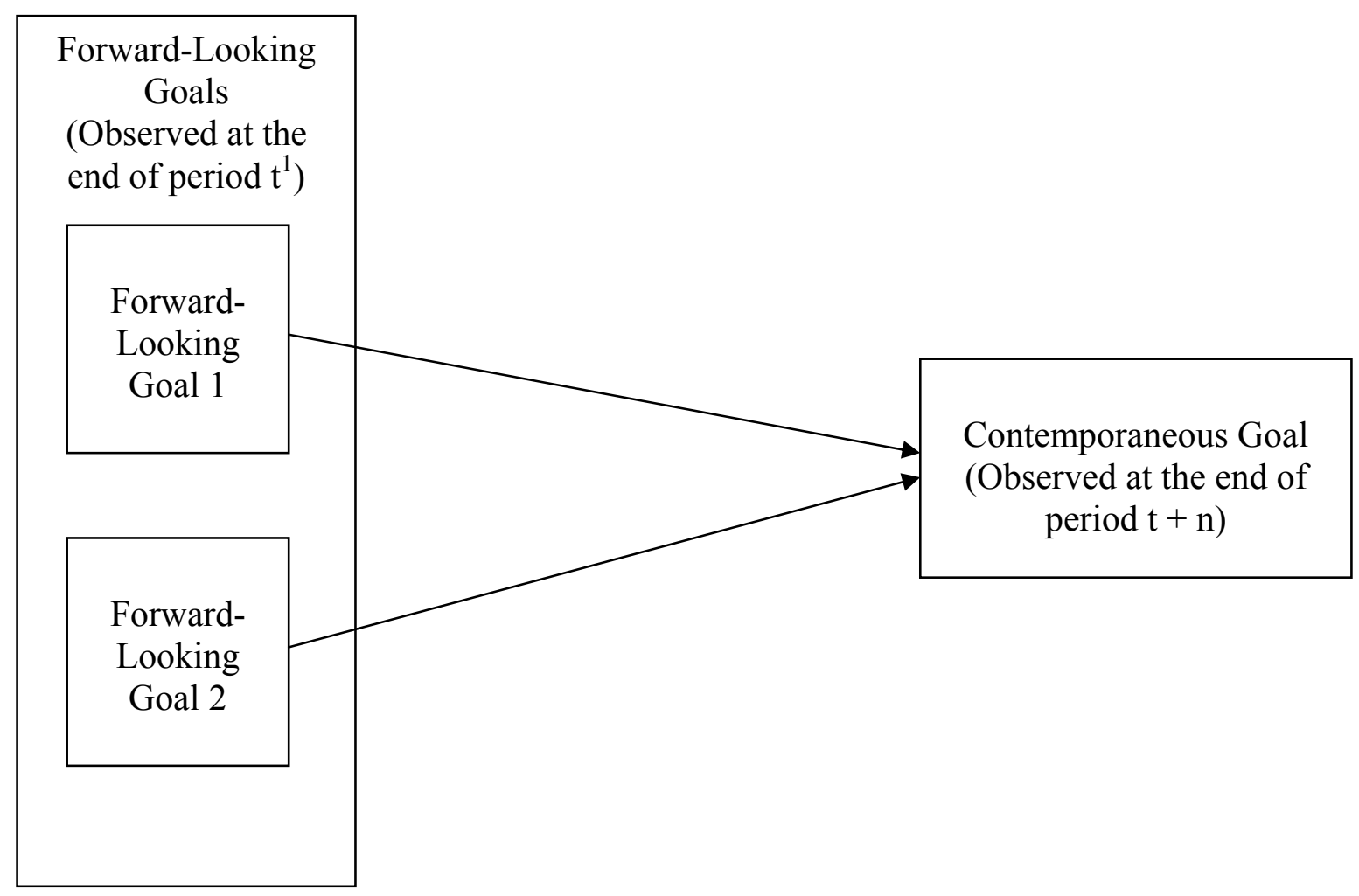

1 Forward-looking goal performance in the current period $(t)$ influences future contemporaneous goal performance (period $t+n$ 
Figure 2

Theoretical Relationships Introduced in Chapter 2

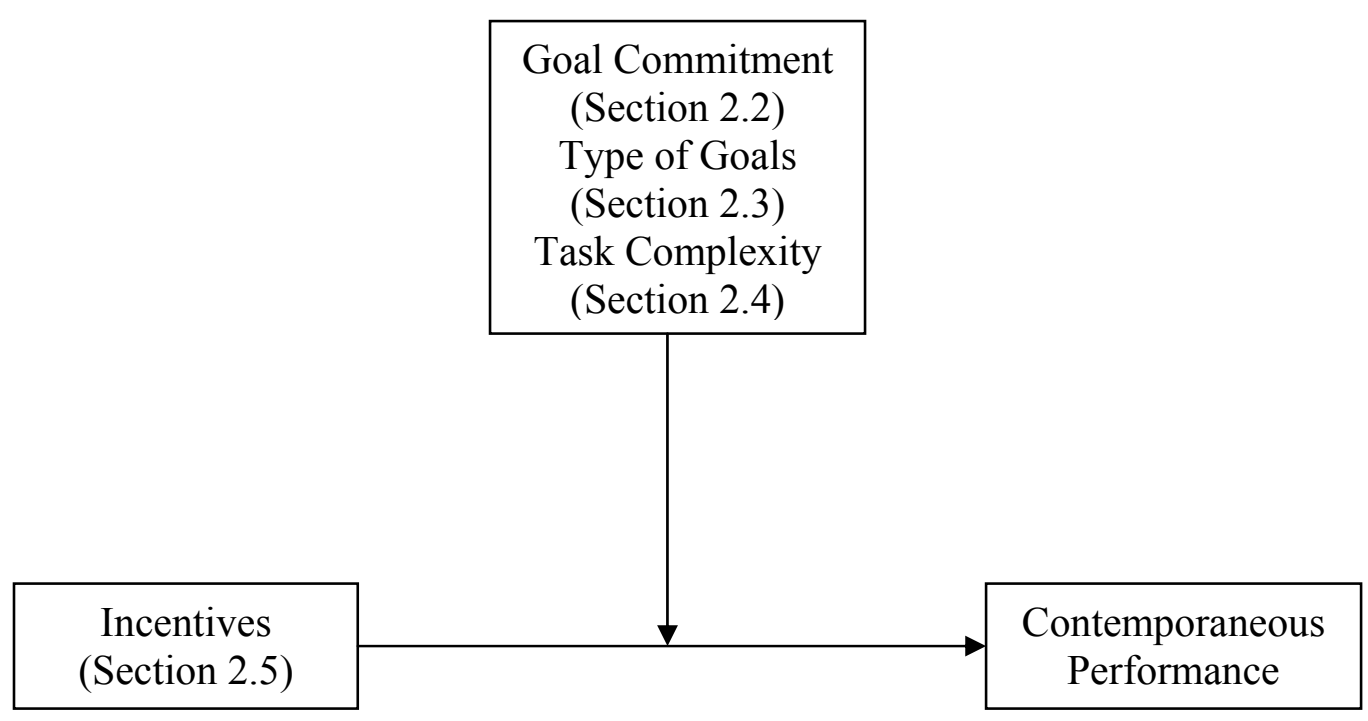


Figure 3

Experimental Design

\begin{tabular}{|c|c|c|c|}
\hline $\begin{array}{r}\text { Incentive } \\
\text { Contract }^{1}\end{array}$ & $\begin{array}{r}\text { Low } \\
\text { Contemporaneous } \\
\text { Weight }^{3}\end{array}$ & $\begin{array}{c}\text { Medium } \\
\text { Contemporaneous }^{4} \\
\text { Weight }^{4}\end{array}$ & $\begin{array}{c}\text { High } \\
\text { Contemporaneous }^{\text {Weight }^{5}}\end{array}$ \\
\hline $\begin{array}{c}\text { Low Complexity } \\
\text { Environment }\end{array}$ & $\mathrm{A}$ & $\mathrm{B}$ & $\mathrm{C}$ \\
\hline $\begin{array}{c}\text { High Complexity } \\
\text { Environment }\end{array}$ & $\mathrm{D}$ & $\mathrm{E}$ & $\mathrm{F}$ \\
\hline
\end{tabular}

1 Incentives are rewarded for attainment of only the contemporaneous goal, or for both the forwardlooking and contemporaneous goals.

2 Participants operate in a business environment of either low or high complexity.

3 When participants earn the maximum bonus available, forward-looking goal attainment is rewarded with $2 / 3$ of the incentives available while contemporaneous goal attainment is rewarded with $1 / 3$ of the incentive available.

4

When participants earn the maximum bonus available, forward-looking goal attainment is rewarded

with, reward $1 / 3$ of incentives available while contemporaneous goal attainment is rewarded with $2 / 3$ of the incentives available.

5

When participants earn the maximum bonus available, only contemporaneous goal attainment is rewarded. 
Figure 4

Hypothesized Relation between Incentive Contract Weights and Investment Decision Quality (Low Complexity)

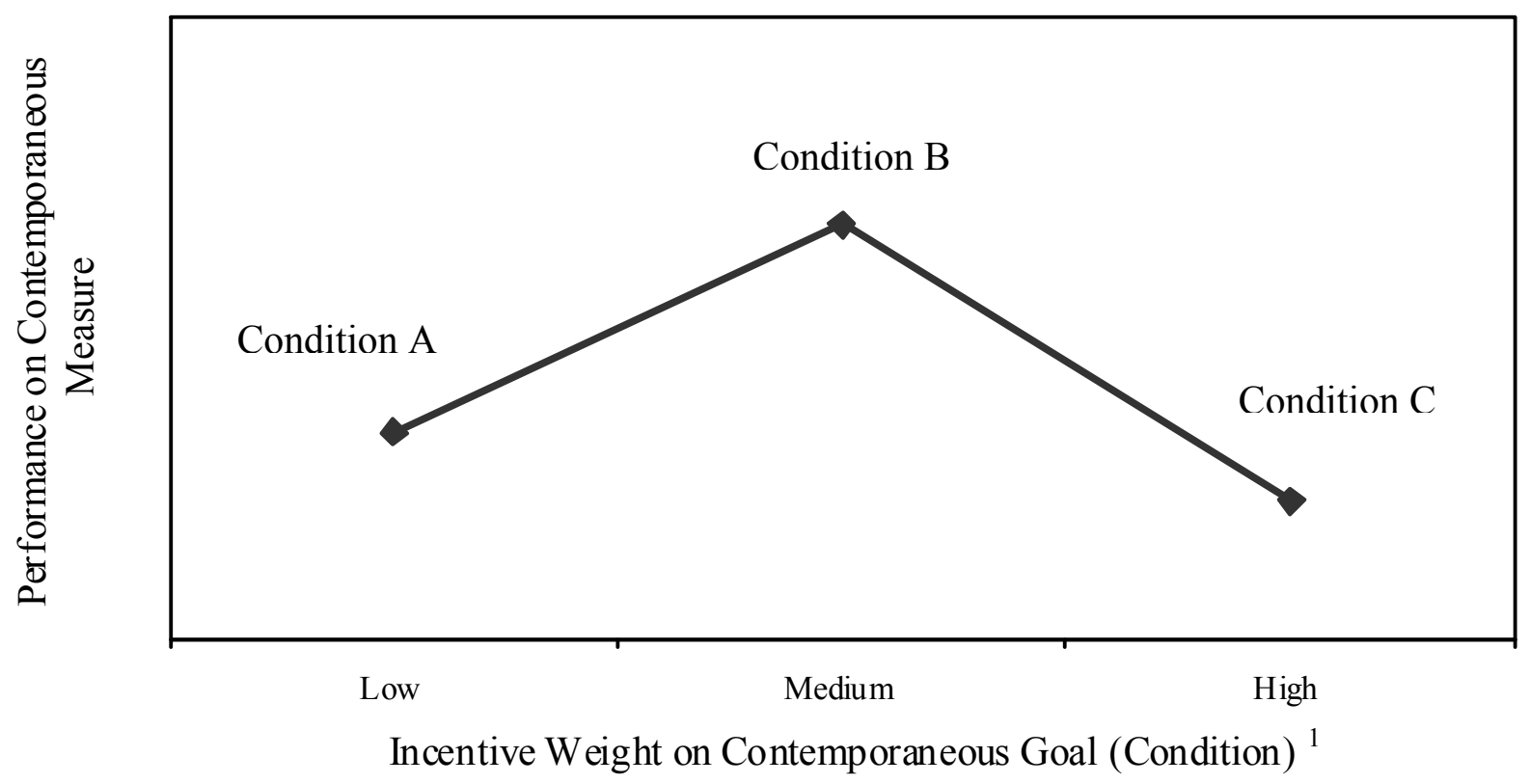

1 In the low incentive condition, when participants earn the maximum bonus available, forward-looking goal attainment is rewarded with $2 / 3$ of the incentives available while contemporaneous goal attainment is rewarded with $1 / 3$ of the incentive available. In the medium incentive condition, when participants earn the maximum bonus available, forward-looking goal attainment is rewarded with, reward 1/3 of incentives available while contemporaneous goal attainment is rewarded with $2 / 3$ of the incentives available. In the high incentive condition, when participants earn the maximum bonus available, only contemporaneous goal attainment is rewarded. 
Figure 5

Hypothesized Mediation Relationship between Incentive Contract Weights and Investment Decision Quality (Low Complexity)

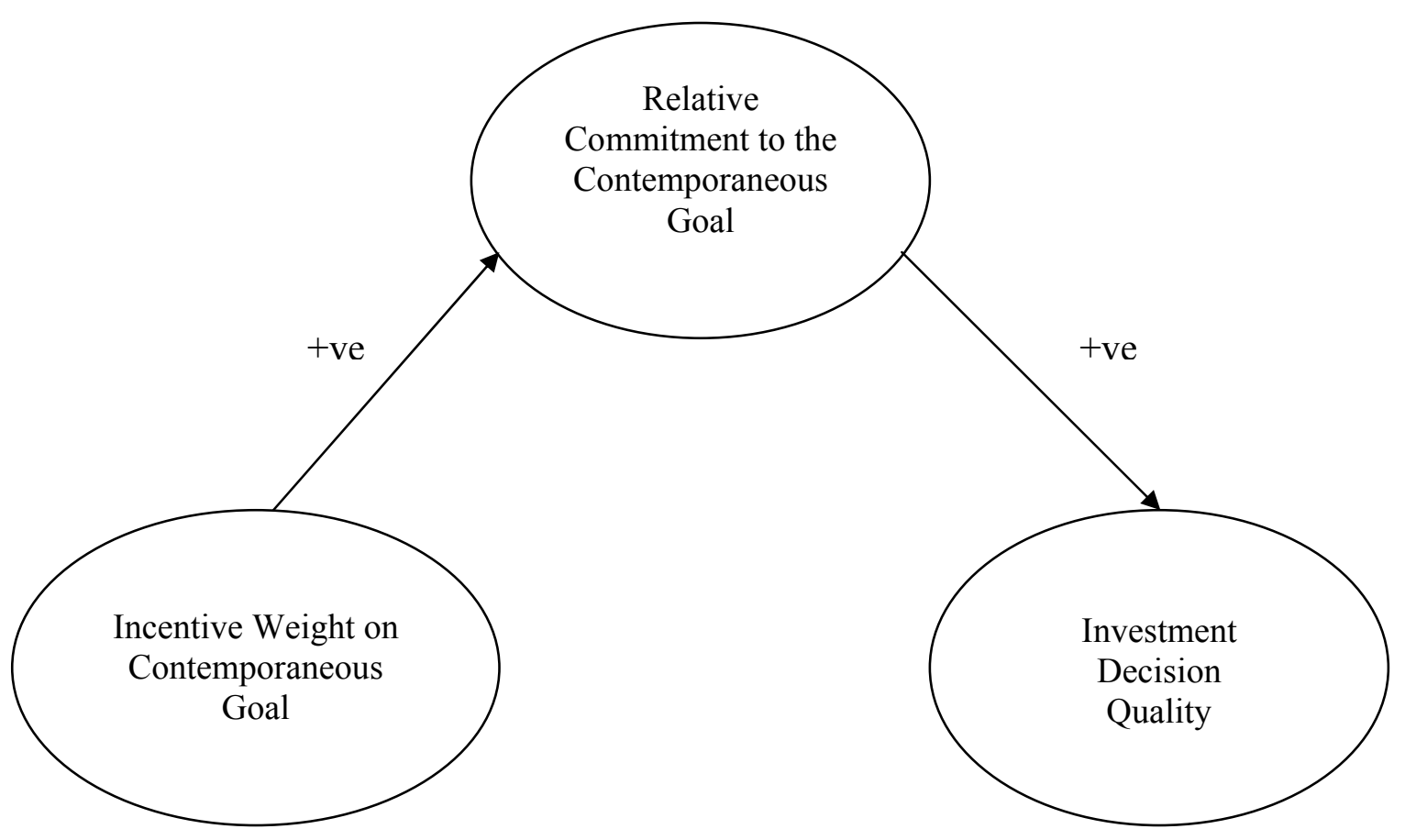


Figure 6

Hypothesized Relation between Incentive Contract Weights and Investment Decision Quality (High Complexity)

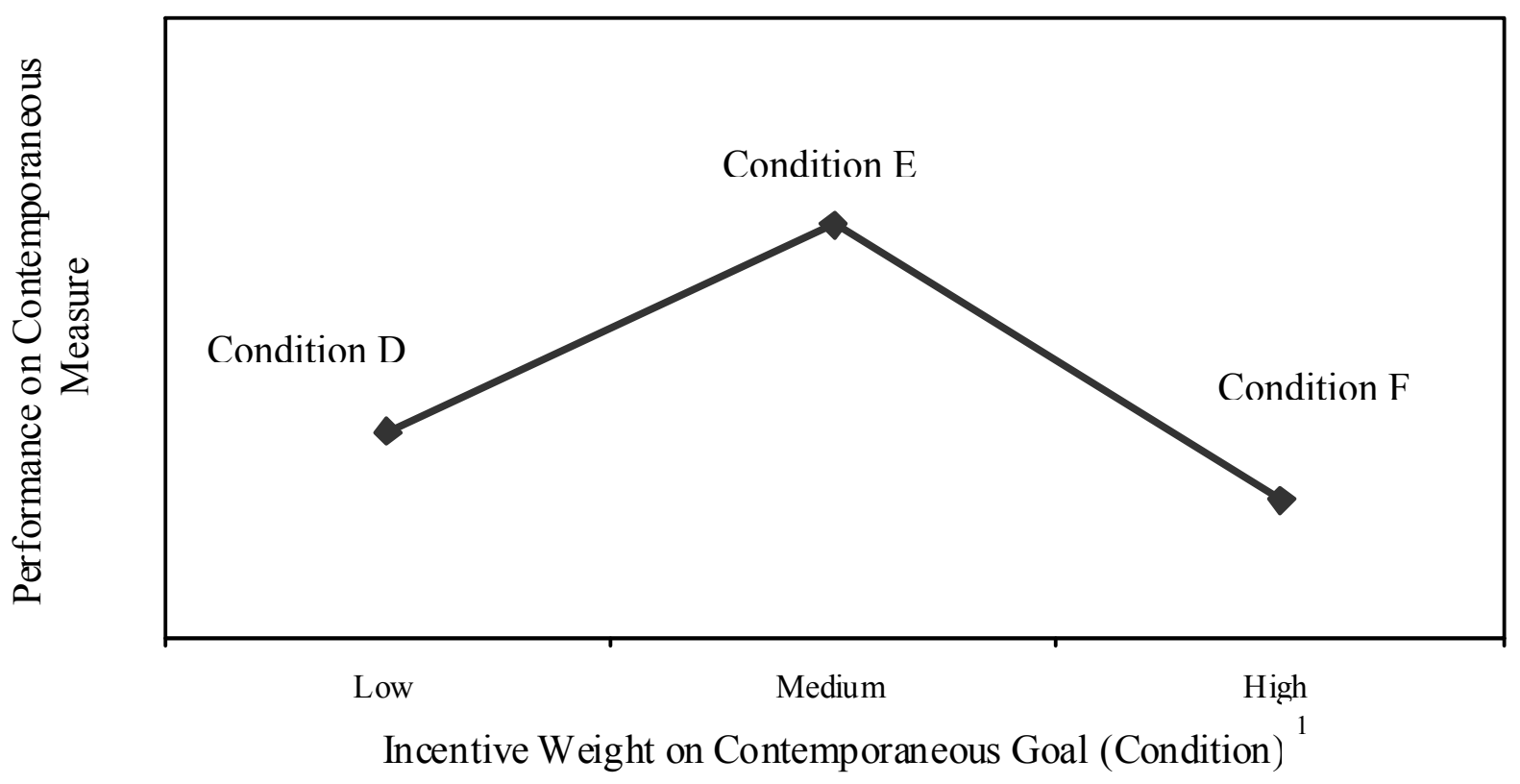

1 In the low incentive condition, when participants earn the maximum bonus available, forward-looking goal attainment is rewarded with $2 / 3$ of the incentives available while contemporaneous goal attainment is rewarded with $1 / 3$ of the incentive available. In the medium incentive condition, when participants earn the maximum bonus available, forward-looking goal attainment is rewarded with, reward $1 / 3$ of incentives available while contemporaneous goal attainment is rewarded with $2 / 3$ of the incentives available. In the high incentive condition, when participants earn the maximum bonus available, only contemporaneous goal attainment is rewarded. 
Figure 7

Hypothesized Mediation Relationship between Incentive Contract Weights and Investment Decision Quality (High Complexity)

Panel A: Comparison of incentive conditions that reward only contemporaneous goal attainment (Condition F) with those that reward both forward-looking and contemporaneous measures (Conditions D and E)

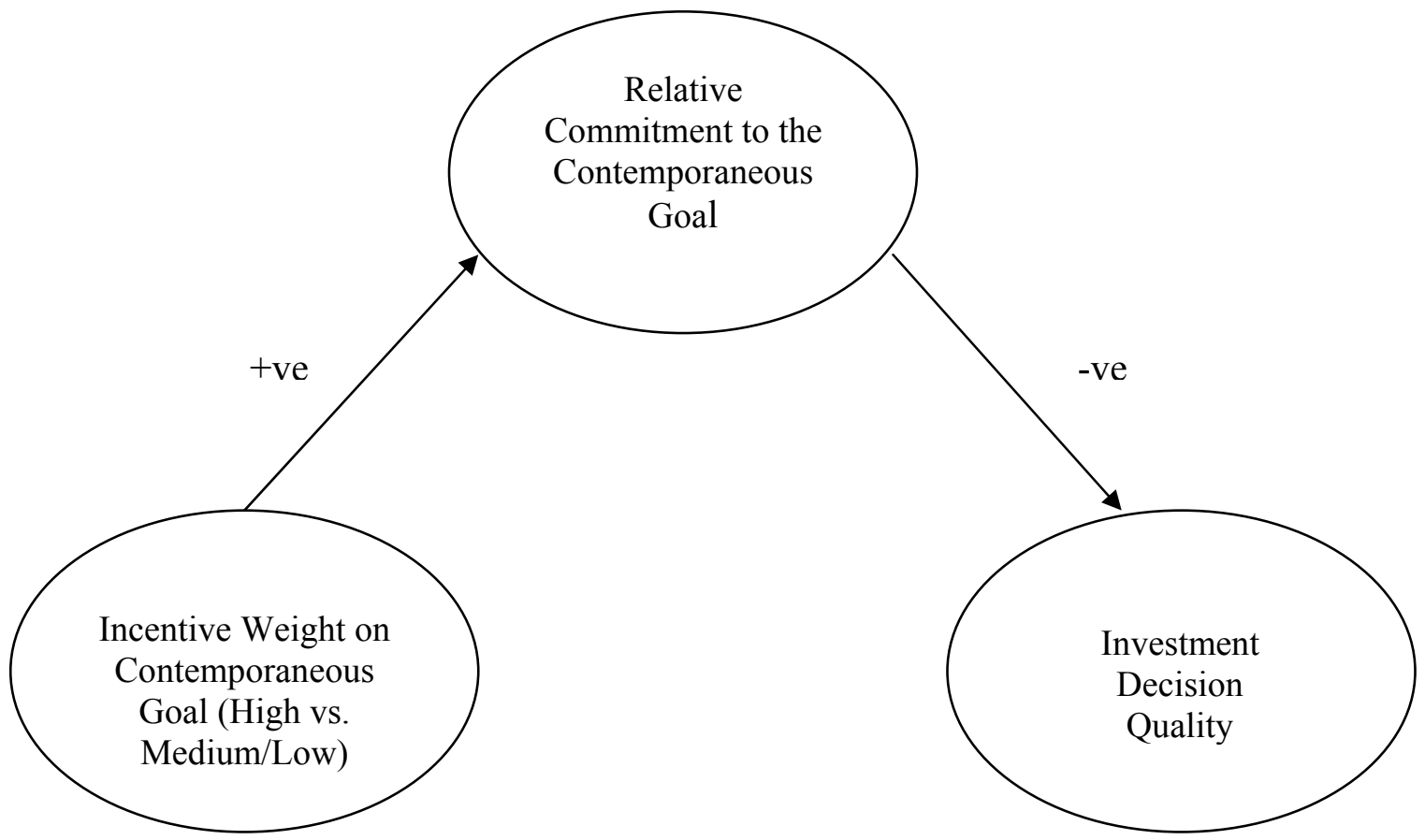

Panel B: Comparison of incentive conditions that reward both forward-looking and contemporaneous measures (Conditions D and E)

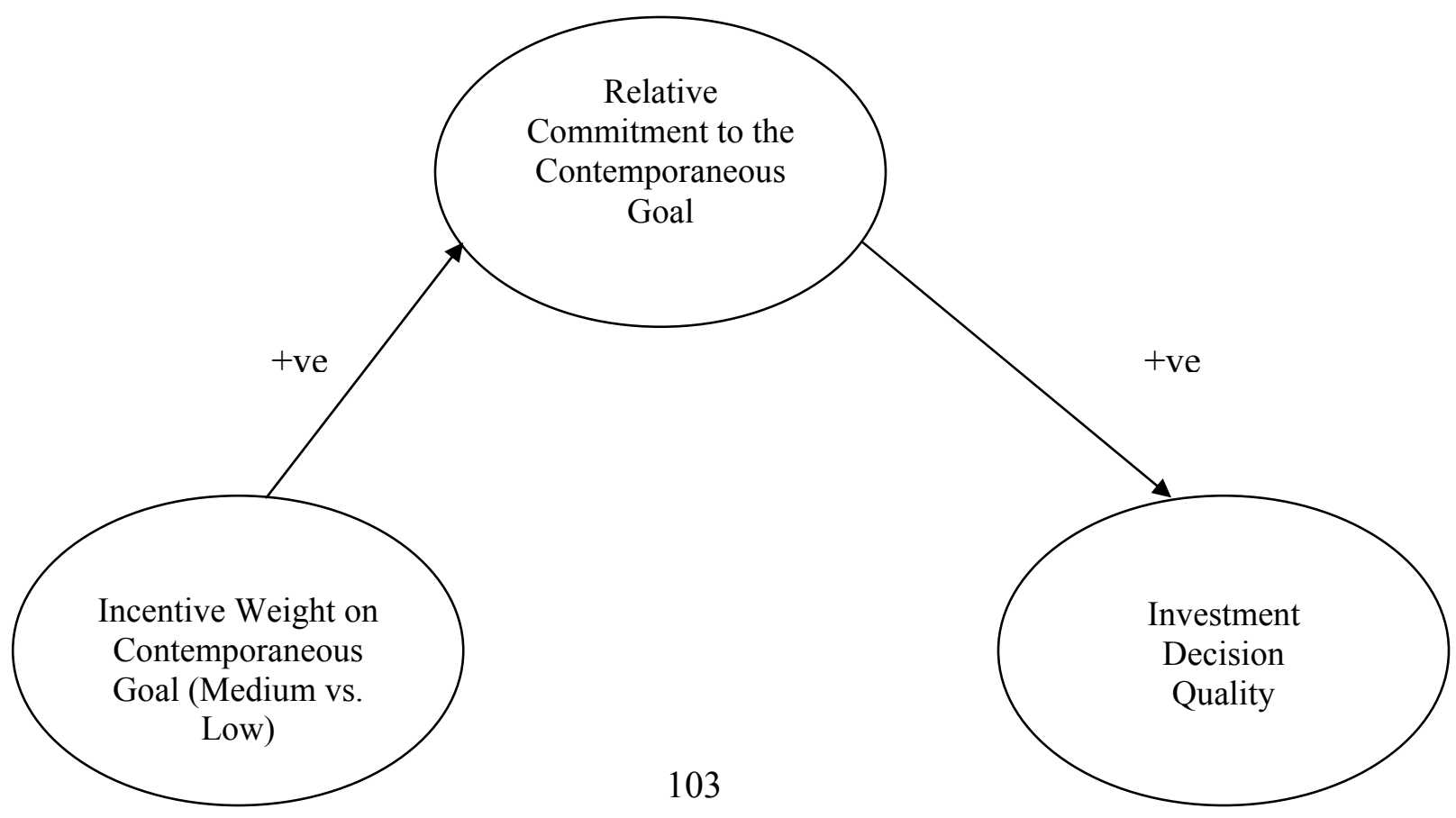


Figure 8

Business Model Employed in the Experimental Simulation

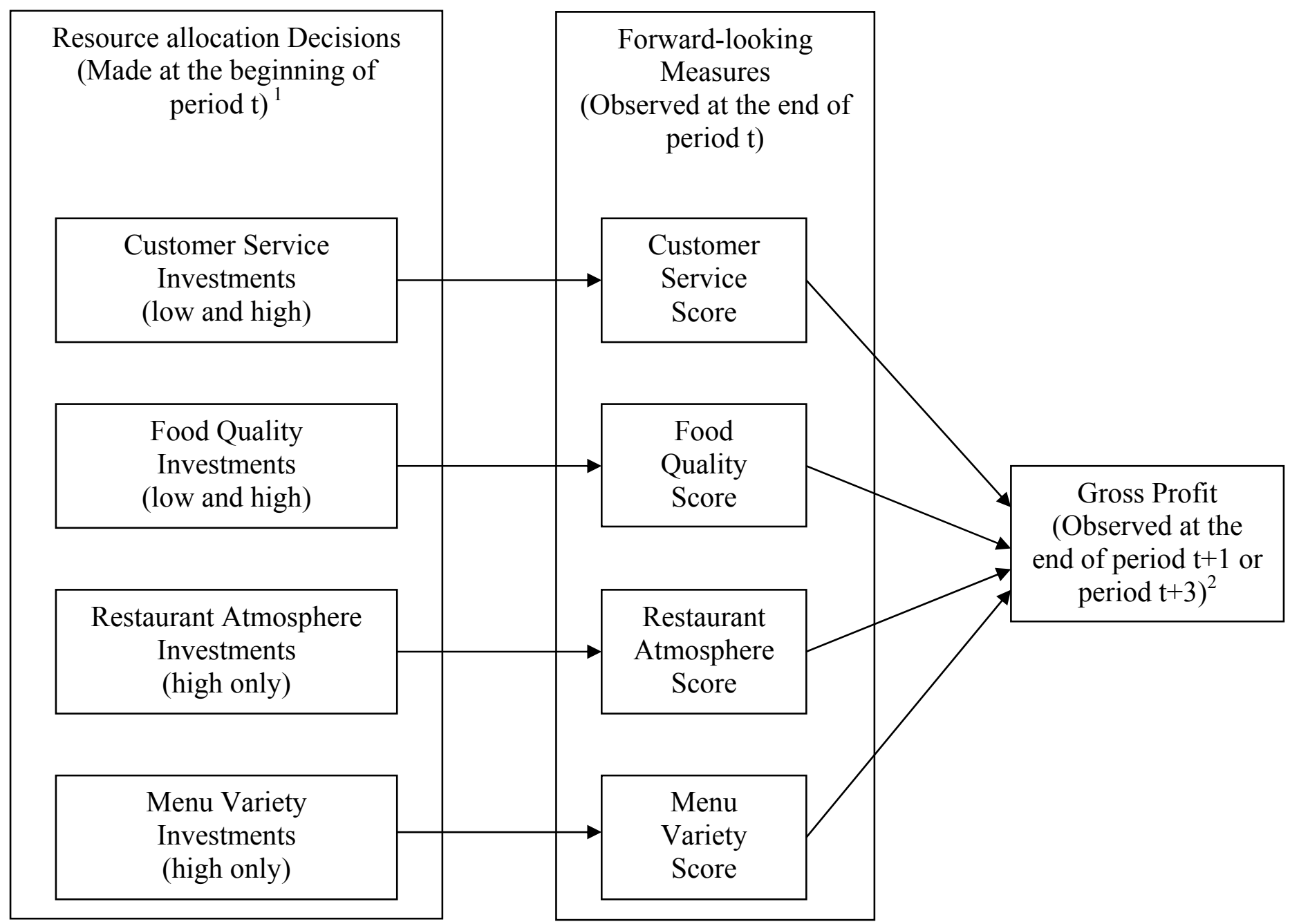


Figure 8 (continued)

1 Restaurant atmosphere and menu variety resource allocation decisions are only included in the high complexity condition.

2 Time lag of $\mathrm{t}+1$ is used in the low complexity condition; time lag of $\mathrm{t}+3$ is used in the high complexity condition. 
Figure 9

Relationship Between Food Quality Investments and Future Sales (High and Low Complexity Conditions)

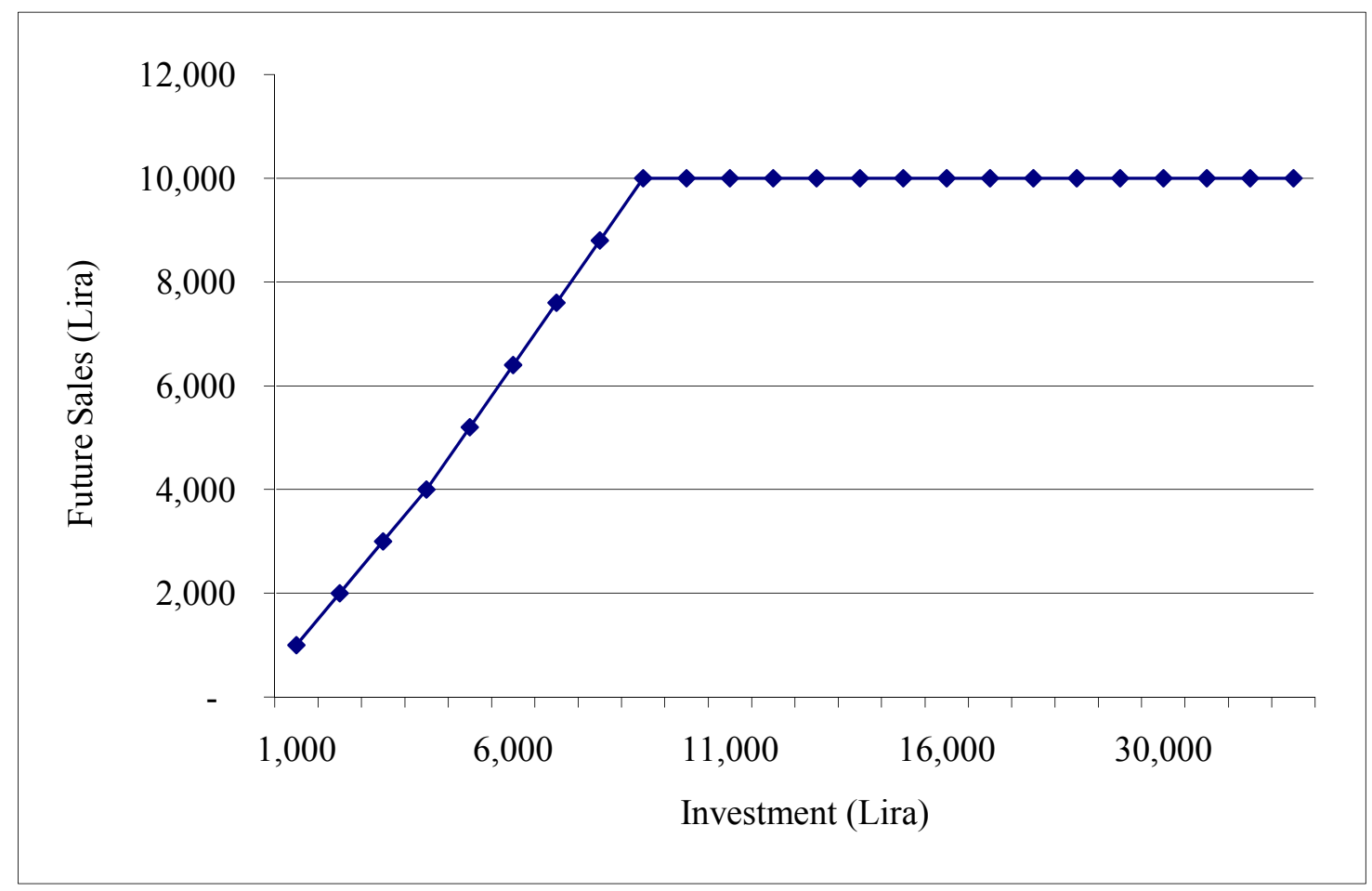


Figure 10

Cumulative Restaurant Net Income Periods 1 - 36

(Low Complexity)

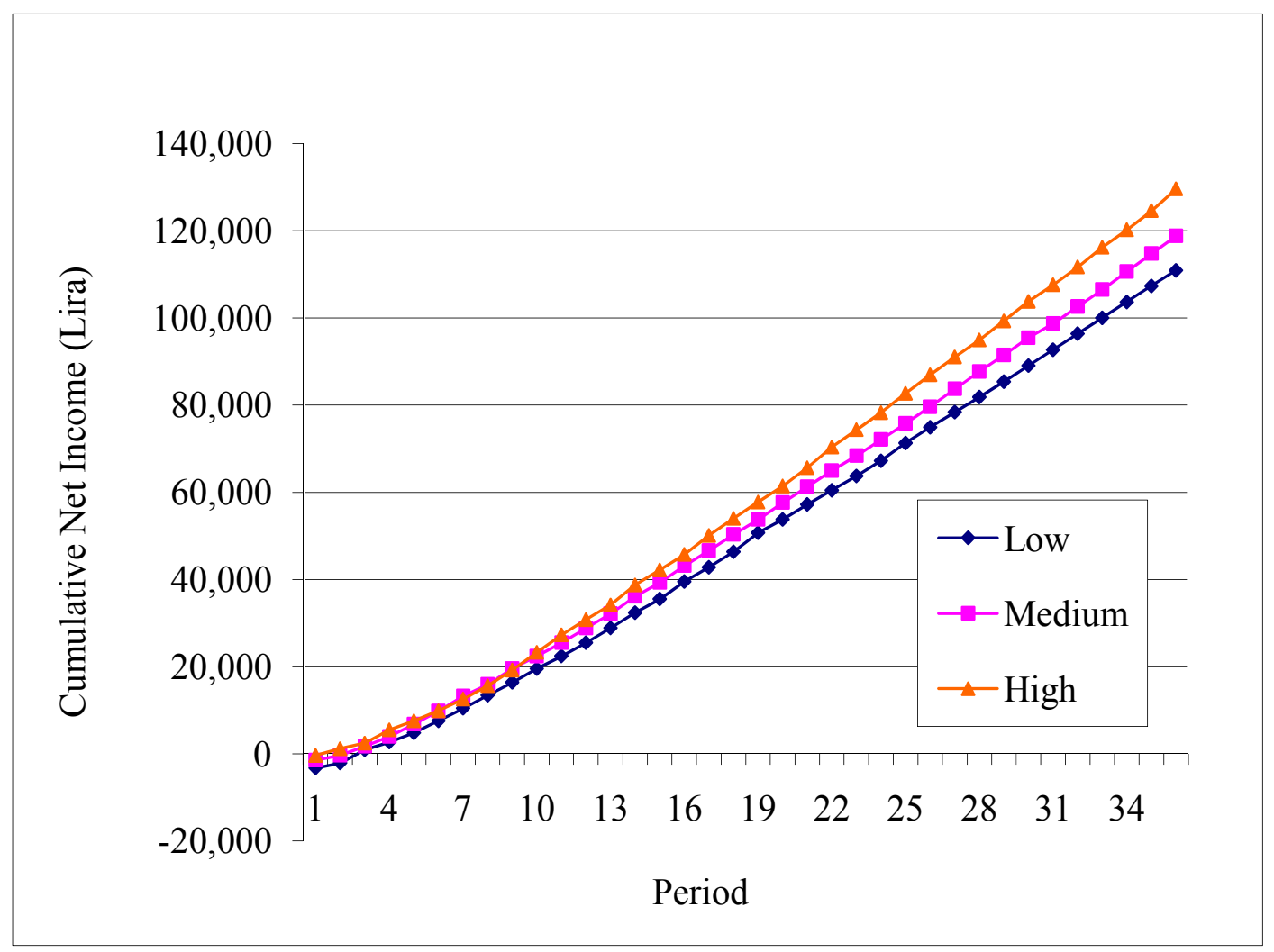


Figure 11

Actual Relation between Incentive Contract Weights and Cumulative Net Income (Low Complexity)

Panel A: Cumulative Income, 36 periods

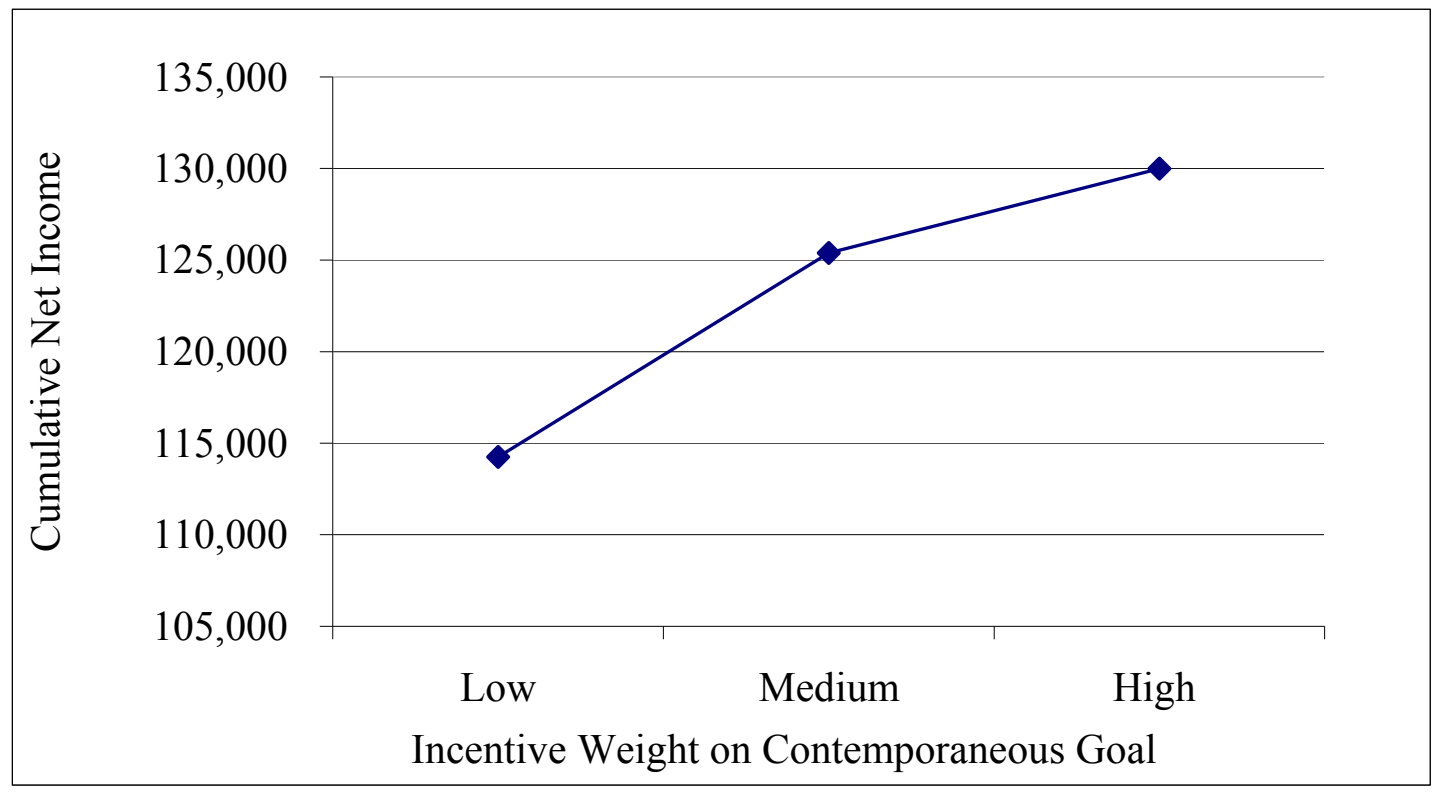

Panel B: Cumulative Income, by 12-Month Period

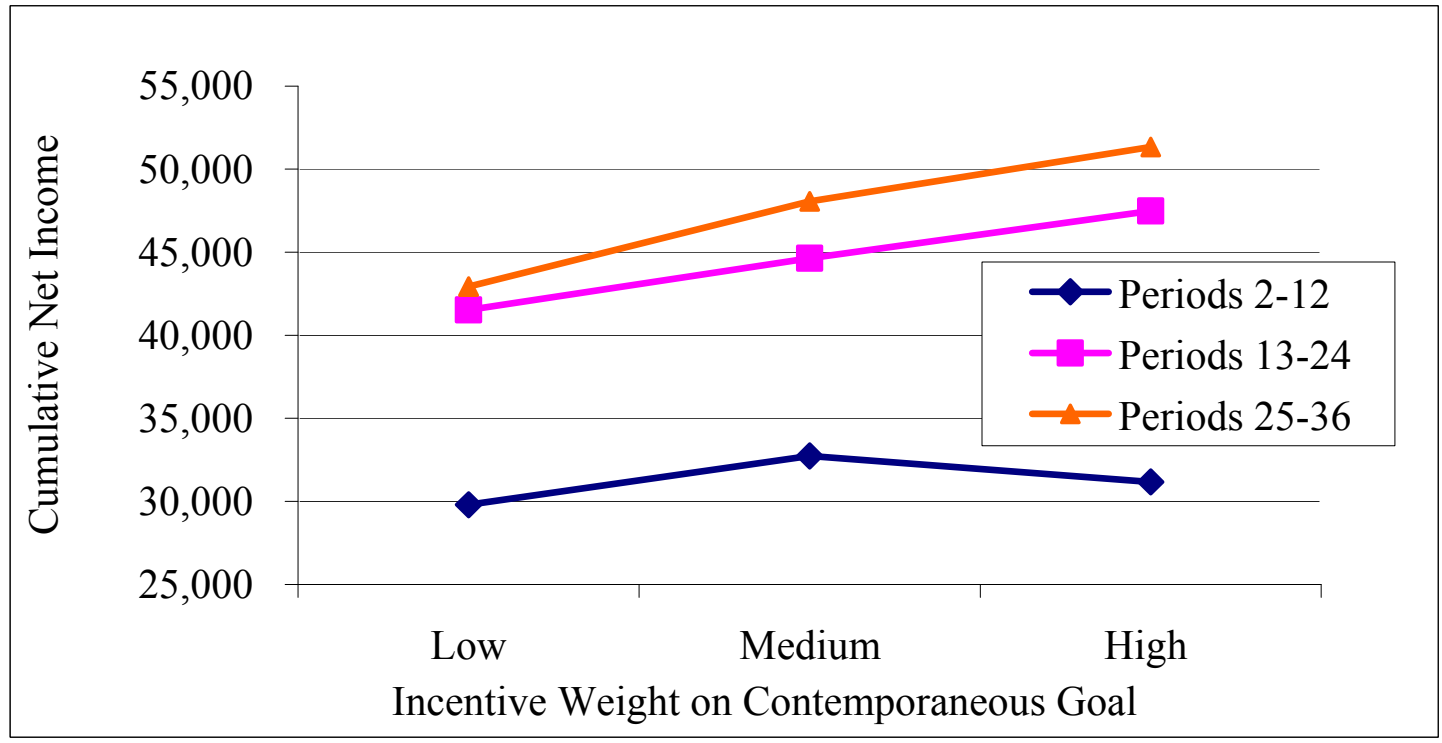


Figure 12

Actual Mediation Relationship between Incentive Contract Weights and Investment Decision Quality (Low Complexity)

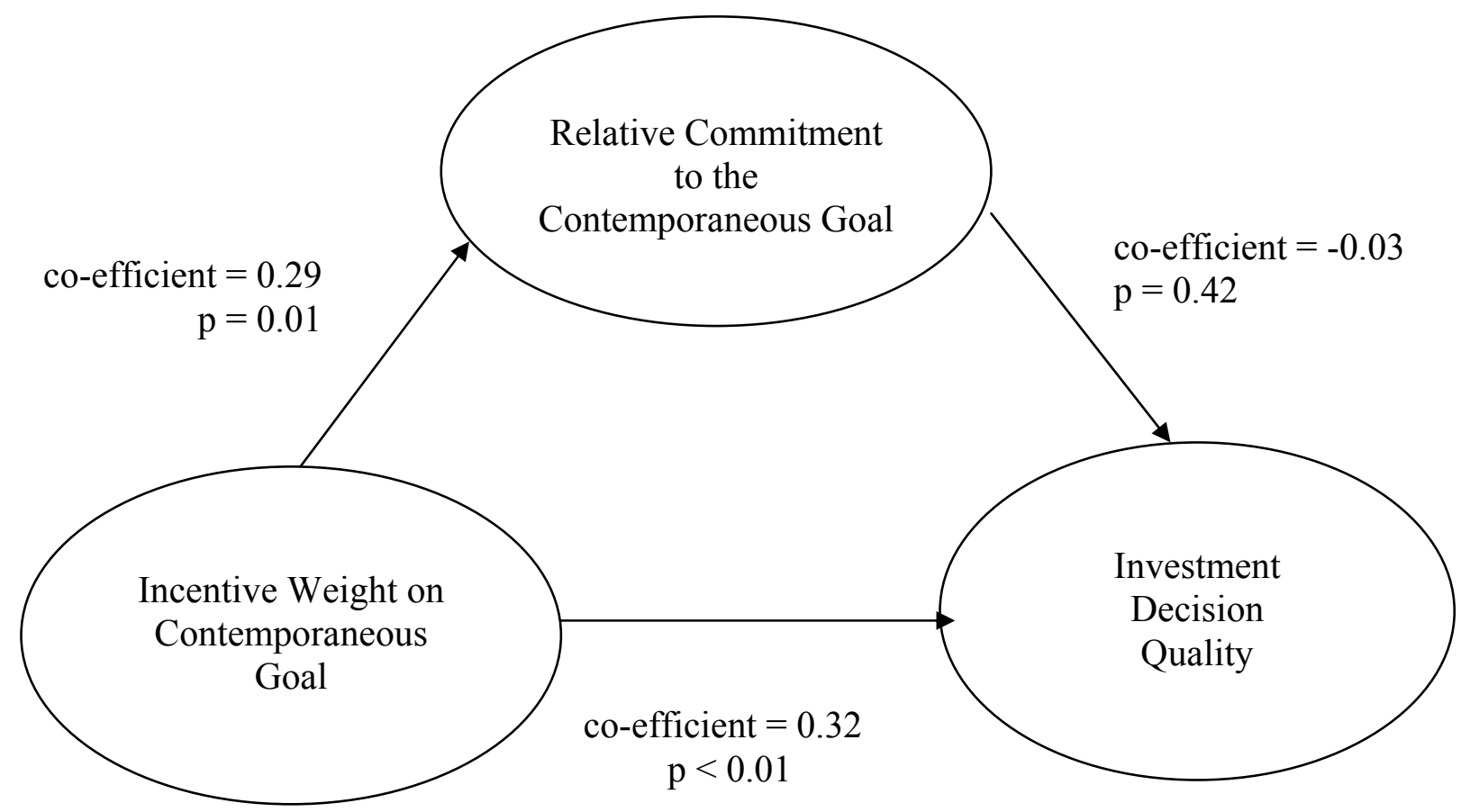


Figure 13

Cumulative Restaurant Net Income Periods

$1-36$ (High Complexity)

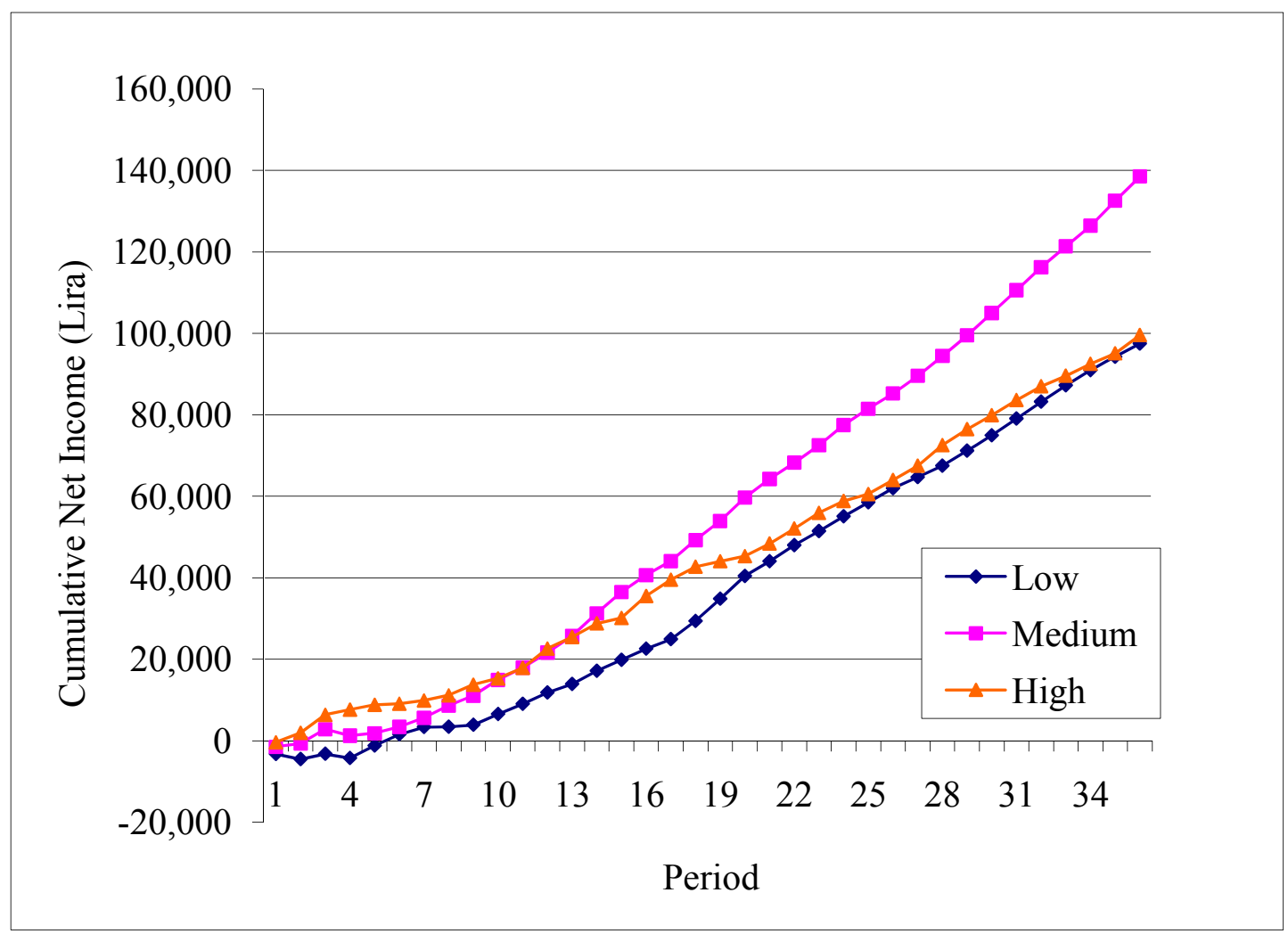


Figure 14

Actual Relation between Incentive Contract Weights and Cumulative Net Income (High Complexity)

Panel A: Cumulative Income, 36 periods

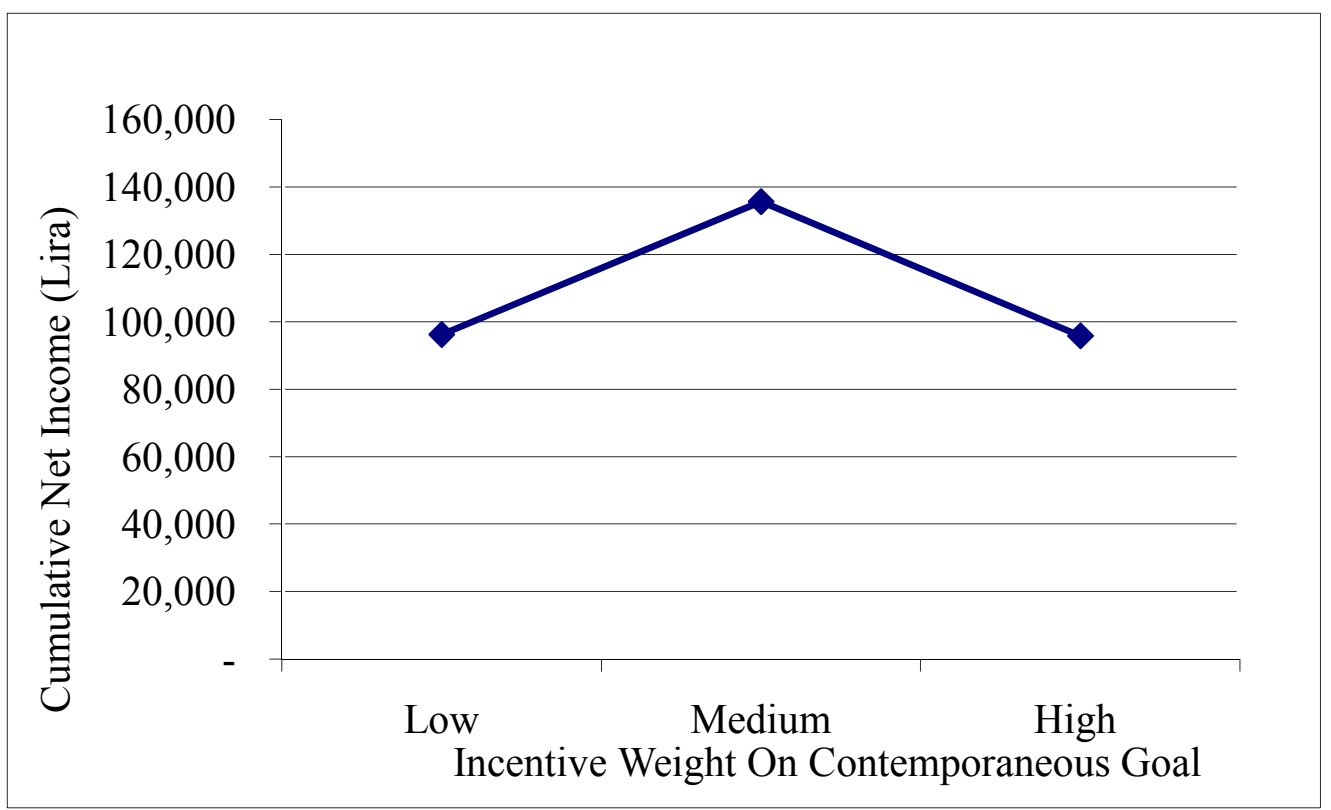

Panel B: Cumulative Income, by 12-Month Period

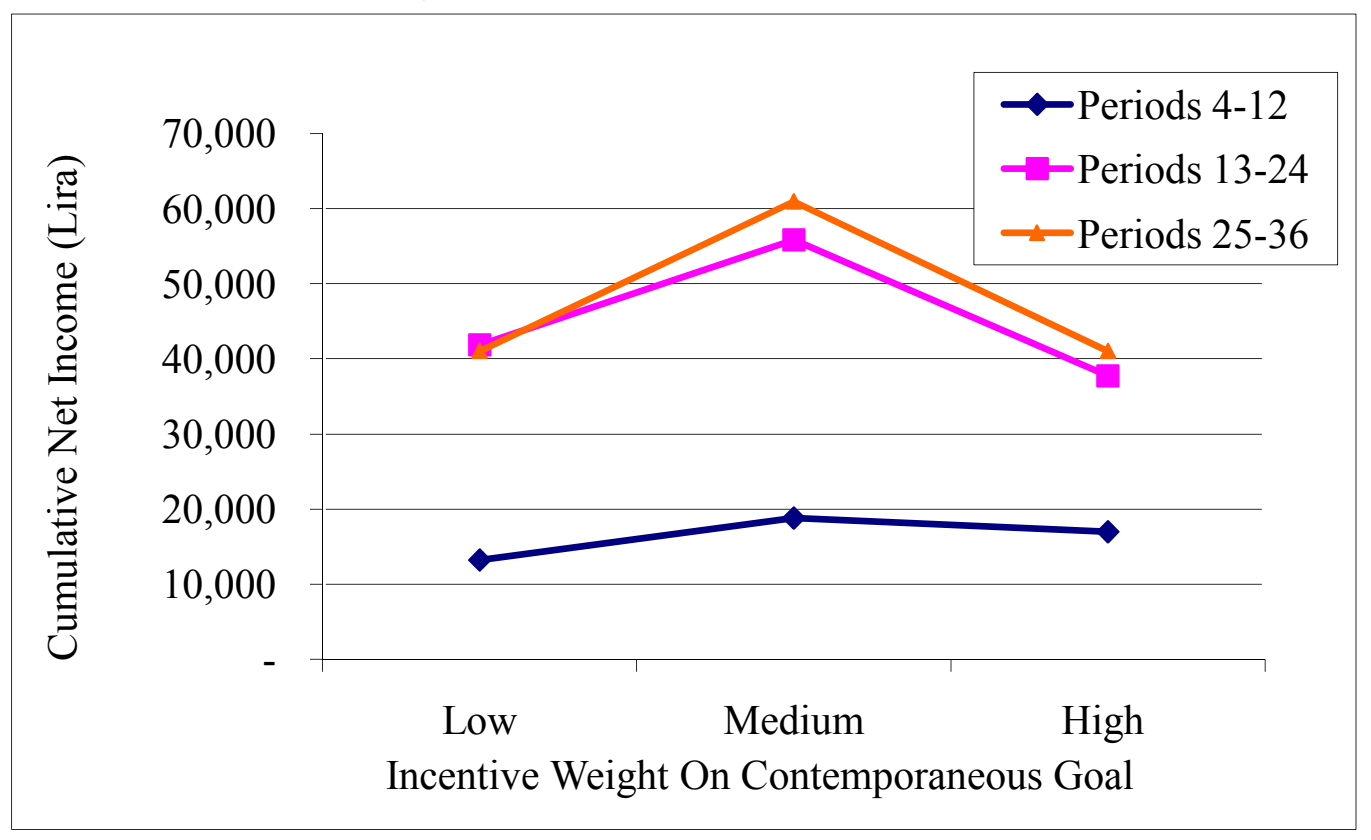


Figure 15

Actual Mediation Relationship between Incentive Contract Weights and Investment Decision Quality (High Complexity)

Panel A: Mediation Analysis, Medium and High Contemporaneous Weights

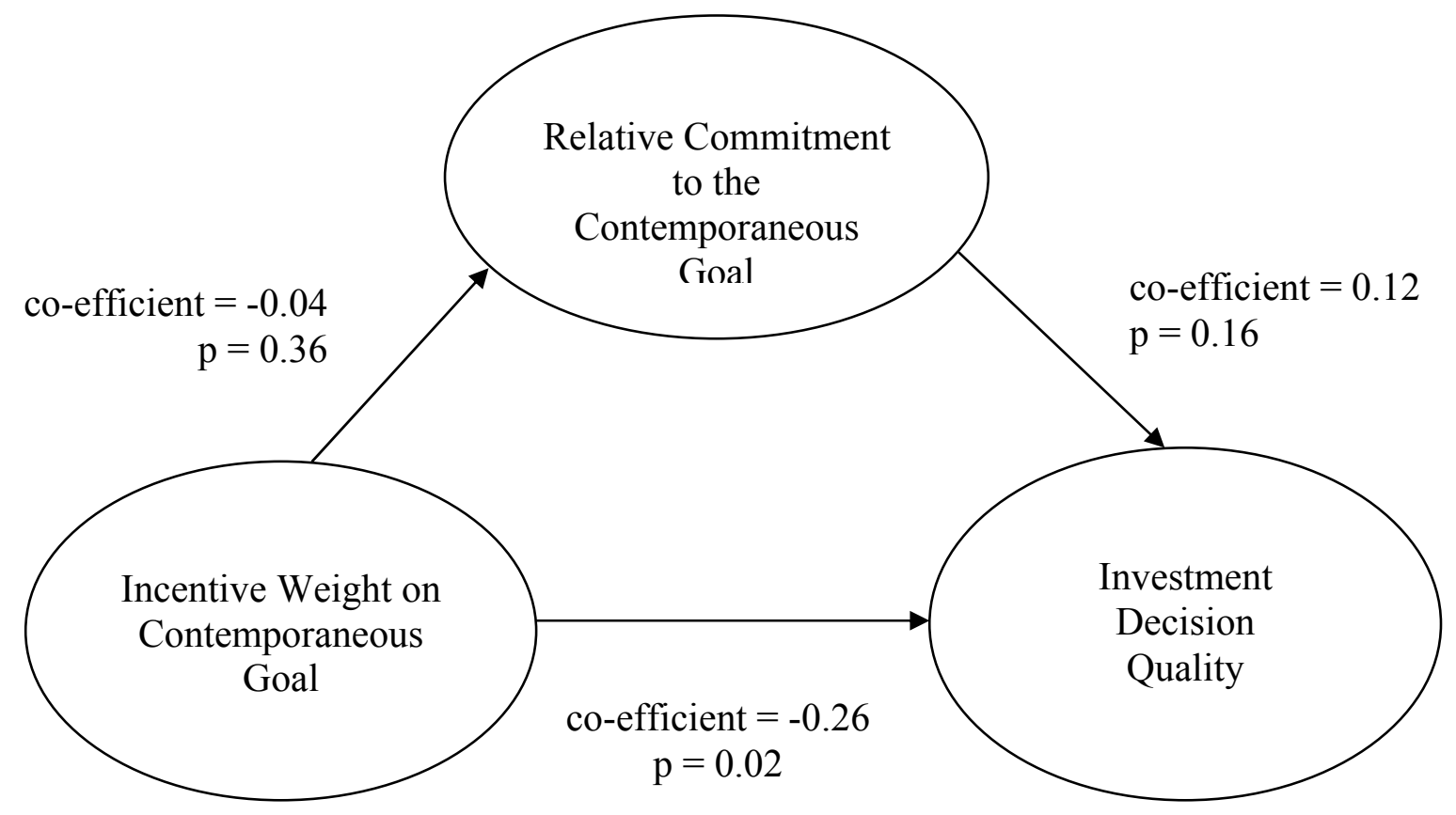

Panel B: Mediation Analysis, Low and Medium Contemporaneous Weights

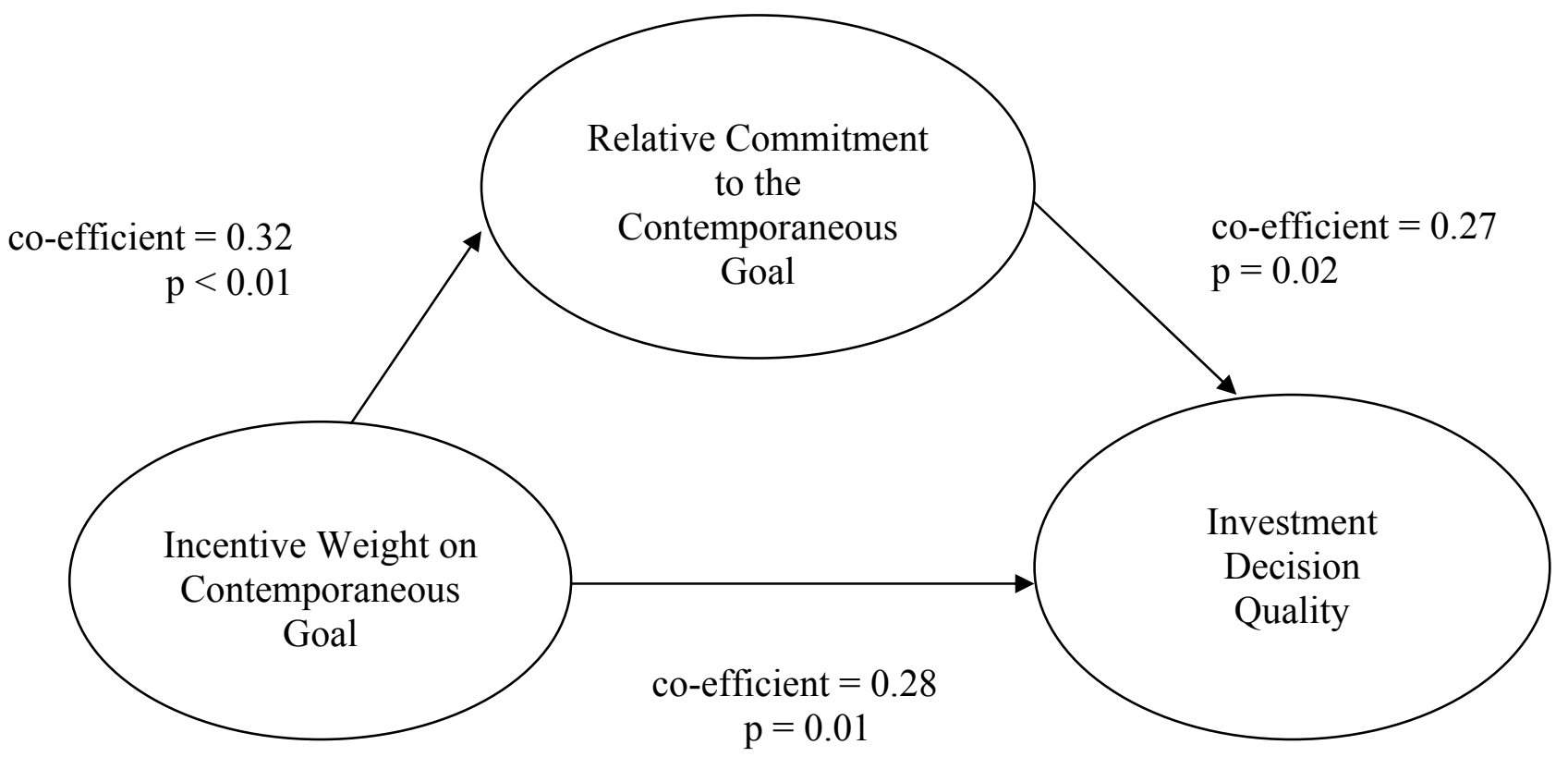




\section{TABLES}

Table 1

Relationships between Resource Allocation Decisions and Gross Income (All currency values expressed in Canadian Lira)

\begin{tabular}{|c|c|c|c|c|c|c|c|c|c|c|c|c|c|c|c|}
\hline \multicolumn{4}{|c|}{ Customer Service $^{1}$} & \multicolumn{4}{|c|}{ Food Quality $^{1}$} & \multicolumn{4}{|c|}{ Restaurant Atmosphere } & \multicolumn{4}{|c|}{ Menu Variety } \\
\hline \begin{tabular}{|c|}
1 \\
Resource \\
Allocation \\
$(\mathrm{t})$ \\
\end{tabular} & \begin{tabular}{|c|}
2 \\
\\
Score \\
$(\mathrm{t})$ \\
\end{tabular} & \begin{tabular}{|c|}
\multicolumn{1}{|c|}{3} \\
Income \\
Effect \\
$(\mathrm{t}+1)$ \\
\end{tabular} & $\begin{array}{c}4 \\
\text { Net } \\
\text { Income } \\
\text { Effect }^{2}\end{array}$ & \begin{tabular}{|c|}
5 \\
Resource \\
Allocation \\
$(\mathrm{t})$ \\
\end{tabular} & $\begin{array}{c}\text { Score } \\
(\mathrm{t})\end{array}$ & \begin{tabular}{l}
$\quad 7$ \\
\multicolumn{1}{c}{7} \\
Income \\
Effect \\
$(\mathrm{t}+1)$ \\
\end{tabular} & $\begin{array}{c}8 \\
\text { Net } \\
\text { Income } \\
\text { Effect }^{2}\end{array}$ & $\begin{array}{c}\text { Resource } \\
\text { Allocation } \\
(\mathrm{t})\end{array}$ & $\begin{array}{c}\text { Score } \\
(\mathrm{t})\end{array} \mid$ & $\begin{array}{l}11 \\
\text { Income } \\
\text { Effect } \\
(\mathrm{t}+1)\end{array}$ & $\begin{array}{c}12 \\
\text { Net } \\
\text { Income } \\
\text { Effect }^{2}\end{array}$ & \begin{tabular}{|c|}
13 \\
Resource \\
Allocation \\
$(\mathrm{t})$ \\
\end{tabular} & $\left|\begin{array}{c}\text { Score } \\
(\mathrm{t})\end{array}\right|$ & \begin{tabular}{|c|} 
\\
15 \\
Income \\
Effect \\
$(\mathrm{t}+1)$ \\
\end{tabular} & $\begin{array}{c}16 \\
\text { Net } \\
\text { Income } \\
\text { Effect }^{2}\end{array}$ \\
\hline $1,000^{3}$ & 3 & 1,200 & 200 & $1,000^{3}$ & 1 & 1,000 & - & $1,000^{3}$ & 1 & 1,000 & - & $1,000^{3}$ & 2 & 1,000 & - \\
\hline $2,000^{3}$ & 4 & 2,400 & 400 & $2,000^{3}$ & 1 & 2,000 & - & $2,000^{3}$ & 1 & 2,000 & - & $2,000^{3}$ & 3 & 2,200 & 200 \\
\hline $3,000^{3}$ & 5 & 3,600 & 600 & $3,000^{3}$ & 2 & 3,000 & - & $3,000^{3}$ & 2 & 3,000 & - & $3,000^{3}$ & 4 & 3,400 & 400 \\
\hline $4,000^{3}$ & 6 & 4,800 & 800 & $4,000^{3}$ & 2 & 4,000 & - & $4,000^{3}$ & 3 & 4,200 & 200 & $4,000^{3}$ & 5 & 4,600 & 600 \\
\hline $5,000^{4}$ & 7 & 6,000 & 1,000 & $5,000^{3}$ & 3 & 5,200 & 200 & $5,000^{3}$ & 4 & 5,400 & 400 & $5,000^{3}$ & 6 & 5,800 & 800 \\
\hline $6,000^{5}$ & 8 & 6,000 & - & $6,000^{3}$ & 4 & 6,400 & 400 & $6,000^{3}$ & 5 & 6,600 & 600 & $6,000^{4}$ & 7 & 7,000 & 1,000 \\
\hline $7,000^{5}$ & 9 & 6,000 & $(1,000)$ & $7,000^{3}$ & 5 & 7,600 & 600 & $7,000^{3}$ & 6 & 7,800 & 800 & $7,000^{5}$ & 8 & 7,000 & - \\
\hline $8,000^{5}$ & 10 & 6,000 & $(2,000)$ & $8,000^{3}$ & 6 & 8,800 & 800 & $8,000^{4}$ & 7 & 9,000 & 1,000 & $8,000^{5}$ & 9 & 7,000 & $(1,000)$ \\
\hline $9,000^{5}$ & 10 & 6,000 & $(3,000)$ & $9,000^{4}$ & 7 & 10,000 & 1,000 & $9,000^{5}$ & 8 & 9,000 & - & $9,000^{5}$ & 10 & 7,000 & $(2,000)$ \\
\hline $10,000^{5}$ & 10 & 6,000 & $(4,000)$ & $10,000^{5}$ & 8 & 10,000 & - & $10,000^{5}$ & 9 & 9,000 & $(1,000)$ & $10,000^{5}$ & 10 & 7,000 & $(3,000)$ \\
\hline $11,000^{5}$ & 10 & 6,000 & & $11,000^{5}$ & 9 & 10,000 & $(1,000)$ & $11,000^{5}$ & 10 & 9,000 & $(2,000)$ & $11,000^{5}$ & 10 & 7,000 & $(4,000)$ \\
\hline $12,000^{5}$ & 10 & 6,000 & & $12,000^{5}$ & 10 & 10,000 & $(2,000)$ & $12,000^{5}$ & 10 & 9,000 & $(3,000)$ & $12,000^{5}$ & 10 & 7,000 & $(5,000)$ \\
\hline $13,000^{5}$ & 10 & 6,000 & $(5,000)$ & $13,000^{5}$ & 10 & 10,000 & $(3,000)$ & $13,000^{5}$ & 10 & 9,000 & $(4,000)$ & $13,000^{5}$ & 10 & 7,000 & $(6,000)$ \\
\hline $14,000^{5}$ & 10 & 6,000 & & $14,000^{5}$ & 10 & 10,000 & $(4,000)$ & $14,000^{5}$ & 10 & 9,000 & $(5,000)$ & $14,000^{5}$ & 10 & 7,000 & $(7,000)$ \\
\hline $15,000^{5}$ & 10 & 6,000 & $(6,000)$ & $15,000^{5}$ & 10 & 10,000 & $(5,000)$ & $15,000^{5}$ & 10 & 9,000 & $(6,000)$ & $15,000^{5}$ & 10 & 7,000 & $(8,000)$ \\
\hline$\cdots$ & $\cdots$ & $\cdots$ & $\cdots$ & $\ldots$ & $\ldots$ & $\cdots$ & $\ldots$ & $\ldots$ & $\ldots$ & $\ldots$ & $\ldots$ & $\cdots$ & $\ldots$ & $\cdots$ & \\
\hline $40,000^{5}$ & 10 & 6,000 & $(34,000)$ & $40,000^{5}$ & 10 & 10,000 & $(30,000)$ & $40,000^{5}$ & 10 & 9,000 & $(31,000)$ & $40,000^{5}$ & 10 & 6,000 & $(34,000)$ \\
\hline
\end{tabular}




\section{Table 1 (Continued)}

Forward-looking measures used in the low complexity conditions where maximum spending was 20,000 Lira.

2 Net Income Effect $=$ Income Effect (column 3, 7, 11 or 15) - Resource Allocation Investment (column 1, 5, 9 or 13)

3 Resource allocation decisions that positively contributed to the attainment of the contemporaneous goal 3,100 (low complexity) or 5,100 (high complexity)

4 Resource allocation that results in attainment of all assigned goals (forward-looking and contemporaneous).

5 Resource allocation decisions that result in the attainment of the forward-looking goal of 7 
Table 2

Example Net Income Calculations

(All currency values expressed in Canadian Lira)

\section{Panel A: Low Complexity Conditions}

\begin{tabular}{|l|c|c|c|c|}
\hline \multirow{2}{*}{} & \multicolumn{4}{|c|}{$\begin{array}{c}\text { Monthly } \\
\text { (in Lira) }\end{array}$} \\
\cline { 2 - 5 } & & $2,000 /$ & $5,000 /$ & $10,000 /$ \\
\cline { 2 - 5 } & $0 / 0^{1}$ & $6,000^{2}$ & $9,000^{3}$ & $10,000^{4}$ \\
\hline Gross Income (Sales less CofS) without Investments & 3,000 & 3,000 & 3,000 & 3,000 \\
Lagged Effect of Customer service ( $\mathrm{t}+1)$ & 0 & 2,400 & 6,000 & 6,000 \\
Lagged Effect of Food Quality ( $\mathrm{t}+1)$ & 0 & 6,700 & 10,000 & 10,000 \\
Actual Gross Income & 3,000 & 12,100 & 19,000 & 19,000 \\
Less: Current Month's Total Investment & 0 & 8,000 & 14,000 & 20,000 \\
\hline Maximum Net Income Per Month & 3,000 & 4,100 & 5,000 & $-1,000$ \\
\hline
\end{tabular}

Panel B: High Environmental Complexity Conditions

\begin{tabular}{|l|c|c|c|c|}
\hline \multirow{2}{*}{} & \multicolumn{4}{|c|}{$\begin{array}{c}\text { Monthly } \\
\text { Satisfaction/Quality/Atmosphere/Variety } \\
\text { Investments (in Lira) }\end{array}$} \\
\cline { 2 - 5 } & & $2,000 /$ & $5,000 /$ & $10,000 /$ \\
& & $6,000 /$ & $9,000 /$ & $10,000 /$ \\
Gross Income (Sales less CofS) without Investments & $3,000 / 0 / 0^{1}$ & $4,000^{2}$ & $6,000 /$ & 10,000 \\
Lagged Effect of Customer service (t+1) & 0 & 3,000 & 3,000 & 3,000 \\
Lagged Effect of Food Quality ( $t+1)$ & 0 & 6,400 & 10,000 & 10,000 \\
Lagged Effect of Restaurant Atmosphere ( $t+1)$ & 0 & 7,800 & 9,000 & 9,000 \\
Lagged Effect of Menu variety (t+1) & 0 & 4,600 & 7,000 & 7,000 \\
\cline { 2 - 5 } Actual Gross Income & 3,000 & 24,200 & 35,000 & 35,000 \\
Less: Current Month's Total Investment & 0 & 19,000 & 28,000 & 40,000 \\
\cline { 2 - 5 } Maximum Net Income Per Month & 3,000 & 5,200 & 7,000 & $-5,000$ \\
\hline
\end{tabular}

1 This resource allocation is an example of a strategy that, if used for two consecutive periods, will not allow participants to attain their contemporaneous (i.e., net income) or forward-looking (i.e., customer service) goals.

2 This resource allocation is an example of a strategy that, if used for two consecutive periods, will allow participants to attain the contemporaneous goal, but not all forward-looking, goals

3 This resource allocation is an example of a strategy that, if used for two consecutive periods, will allow participants to attain all forward-looking and contemporaneous goals.

4 This resource allocation is an example of a strategy that, if used for two consecutive periods, will allow participants to attain all forward-looking, but not contemporaneous, goals. 
Table 3

Incentives Paid for Attaining Assigned Goals

(All currency values expressed in Canadian Lira)

\begin{tabular}{|c|c|c|c|c|c|c|c|c|c|}
\hline \multicolumn{10}{|c|}{ A: Low Complexity Conditions ( I wo Forward-looking Measures) } \\
\hline \multicolumn{2}{|c|}{$\begin{array}{c}\text { Monthly } \\
\text { Performance }\end{array}$} & \multicolumn{3}{|c|}{$\begin{array}{l}\text { 2/3 Forward-looking and } \\
1 / 3 \text { Contemporaneous } \\
\text { Incentive Contract }^{1} \\
\end{array}$} & \multicolumn{3}{|c|}{$\begin{array}{l}\text { 1/3 Forward-looking and } \\
\text { 2/3 Contemporaneous } \\
\text { Incentive Contract }^{2}\end{array}$} & \multicolumn{2}{|c|}{$\begin{array}{c}\text { Contemporaneou: } \\
\text { Measures Only } \\
\text { Incentive } \\
\text { Contract }^{3} \\
\end{array}$} \\
\hline $\begin{array}{l}\text { Forward- } \\
\text { looking } \\
\text { Measure }\end{array}$ & $\begin{array}{c}\text { Net } \\
\text { Income }\end{array}$ & $\begin{array}{l}\text { Forward- } \\
\text { looking } \\
\text { Measure }\end{array}$ & $\begin{array}{l}\text { Net } \\
\text { Income }\end{array}$ & Total $^{5}$ & $\begin{array}{l}\text { Forward- } \\
\text { looking } \\
\text { Measure }\end{array}$ & $\begin{array}{c}\text { Net } \\
\text { Income }\end{array}$ & Total $^{5}$ & $\begin{array}{c}\text { Net } \\
\text { Income }\end{array}$ & Total \\
\hline See note $A$ & 3,100 & See note $A$ & 80 & 80 & See note $A$ & 160 & 160 & 240 & 240 \\
\hline $7^{4}$ & $4,999+{ }^{4}$ & 100 & 100 & 300 & 50 & 200 & 300 & 300 & 300 \\
\hline $8-10$ & See note $B$ & 120 & See note B & 240 & 60 & See note $B$ & 120 & & \\
\hline
\end{tabular}

Panel B: High Complexity Conditions (Four Forward-looking Measures)

\begin{tabular}{|c|c|c|c|c|c|c|c|c|c|}
\hline \multicolumn{2}{|c|}{$\begin{array}{c}\text { Monthly } \\
\text { Performance }\end{array}$} & \multicolumn{3}{|c|}{$\begin{array}{l}2 / 3 \text { Forward-looking and } \\
1 / 3 \text { Contemporaneous } \\
\text { Incentive Contract }^{1}\end{array}$} & \multicolumn{3}{|c|}{$\begin{array}{l}\text { 1/3 Forward-looking and } \\
\text { 2/3 Contemporaneous } \\
\text { Incentive Contract }^{2}\end{array}$} & \multicolumn{2}{|c|}{$\begin{array}{l}\text { Contemporaneous } \\
\text { Measures Only } \\
\text { Incentive } \\
\text { Contract }^{3} \\
\end{array}$} \\
\hline $\begin{array}{l}\text { Forward- } \\
\text { looking } \\
\text { Measure }\end{array}$ & $\begin{array}{c}\text { Net } \\
\text { Income }\end{array}$ & $\begin{array}{l}\text { Forward- } \\
\text { looking } \\
\text { Measure }\end{array}$ & $\begin{array}{c}\text { Net } \\
\text { Income }\end{array}$ & Total $^{6}$ & \begin{tabular}{|l|} 
Forward- \\
looking \\
Measure
\end{tabular} & $\begin{array}{c}\text { Net } \\
\text { Income }\end{array}$ & Total & $\begin{array}{l}\text { Net } \\
\text { Income }\end{array}$ & Total \\
\hline see note $A$ & 5.100 & See note $A$ & 80 & 80 & See note $A$ & 160 & 160 & 240 & 240 \\
\hline $7^{4}$ & $6,999+{ }^{4}$ & 50 & 100 & 300 & 25 & 200 & 300 & 300 & 300 \\
\hline $8-10$ & See note B & 60 & See note B & 240 & 30 & See note B & 120 & & \\
\hline
\end{tabular}

Note A: Different combinations of performance on forward-looking measures will lead to attainment of the net income goal, but not all forward-looking goals. See Table 2 for an example.

Note $B$ : Investments in forward-looking measures that result in monthly performance greater than $7 / 10$ will not result in attainment of the net income goal. See Table 2 for an example.

1 When participants earn the maximum bonus available, forward-looking goal attainment is rewarded with $2 / 3$ of the total incentives available while contemporaneous goal attainment is rewarded with $1 / 3$ of the total incentive available. (Figure 1, Conditions A and D)

2 When participants earn the maximum bonus available, forward-looking goal attainment is rewarded with, reward $1 / 3$ of total incentives available while contemporaneous goal attainment is rewarded with $2 / 3$ of the total incentives available. (Figure 1, Conditions B and E)

3 When participants earn the maximum bonus available, contemporaneous goal attainment is rewarded with all of the incentives available. (Figure 1, Conditions $\mathrm{C}$ and $\mathrm{F}$ )

4 The performance required to maximize the participant's earnings, as well as the long-term performance of the company.

5 Total earnings $=($ Forward-looking Bonus $\times 2)+$ Net Income Bonus

6 Total earnings $=($ Forward-looking Bonus $\mathrm{x} 4)+$ Net Income Bonus 
Table 4

Goal Commitment Measurements

The following questions were used to measure participants' commitment to the forwardlooking and contemporaneous goals after periods 12 and 24 in the simulation.

1. It's hard to take this target seriously $(\mathrm{R})$

2. Quite frankly, I don't care if I achieve this target or not (R)

3. I am strongly committed to pursuing this target

4. It wouldn't take much to make me abandon this target $(\mathrm{R})$

5. I think this is a good target to strive for

Note: For questions marked with an (R), reverse coding is required

Responses for questions are collected using a 7-point Likert scale ranging from -3 (Strongly Disagree) to +3 (Strongly Agree). 


\section{Table 5}

Task Complexity Measurements

The following questions were used to measure participants' perceptions of task complexity after periods 12 and 24 in the simulation.

1 It is difficult to attain the net income goal. ${ }^{1}$

2 It is difficult to attain the non-financial goals. ${ }^{1}$

3 Overall, I find that managing this restaurant is a difficult task. ${ }^{1}$

The following questions were used to measure participants' perceptions of task complexity at the end of the simulation.

4 How difficult did you find the task of investing in customer service, food quality, restaurant atmosphere and menu variety? ${ }^{2}$

5 How difficult did you find the overall task of maximizing your bonus payouts? ${ }^{2}$

6 Many times, I had to check something before I made my investment decisions. ${ }^{2}$

7 I had to think about a lot of different things at the same time to successfully perform this task. ${ }^{2}$

8 The required task was difficult to perform.

9 It was difficult deciding how much to spend on each activity in order to achieve the net income goal each period. ${ }^{1}$

10 It was difficult to determine the impact of the resource allocation decision on net income. ${ }^{1}$

Responses for questions 1-3, and 6-10 are collected using a 7-point Likert scale ranging from -3 (Strongly Disagree) to +3 (Strongly Agree). Responses for questions 4 and 5 were also collected using a 7-point Likert scale ranging from -3 (Very Difficult) to 3 (Very Easy.) 
Table 6

Task Understanding Questions

1. How many months do you have to manage the restaurant?
a. 3 months
b. 6 months
c. 12 months
d. 36 months

2. Which of the following investments will have a positive effect on future earnings? (check all that apply)
a. Customer Service
b. Food Quality
c. Restaurant Atmosphere
d. Menu Variety
e. None of the above

3. What is the maximum amount (in total) that you can spend each month?

4. What is the minimum amount (in total) that you can spend each month?

5. Which of the following goals were you assigned in this simulation? (select all that apply)
a. Net Income of 5,100
b. Customer Service Score of 7
c. Food Quality Score of 7
d. Restaurant Quality Score of 7
e. None of the above

6. Given the following levels of performance, calculate the bonus you would earn based on your monthly Net Income result. (Hint: Consider only your bonus payment, not your monthly salary, when answering this question.)

Net Income of 3,000 Lira

Net Income of 5,100 Lira

Net Income of 7,000 Lira

7. Given the following levels of performance, calculate the bonus you would earn at the end of the month. (Hint: Do not include your monthly salary in your answer.)
a. 0 Lira
b. 160 Lira
c. 240 Lira
d. 300 Lira

8. If the restaurant received a customer service score of 7 , a food quality score of 7 , a menu variety score of 7 and a Net Income score of 5,500 Lira, calculate the bonus you would earn at the end of each month. 
Table 7

Summary of Experimental Procedures

Each participant in this study participated in the following procedures:

1. Participants arrived at the designated on-campus computer lab.

2. Participants were assigned a computer terminal by the experimental facilitator.

3. Participants read and signed the information letter required by the university's human research ethics policies.

4. Participants were assigned an identification number by the experiment facilitator.

5. Participants logged onto the computer simulation.

6. The computer simulation randomly assigned each participant to one of the experimental conditions.

7. In the computer simulation, participants read information about their restaurant, their responsibilities as restaurant manager, their time horizon, and forward-looking measures (e.g., customer satisfaction) important to the restaurant business.

8. Participants provided their assessment of how each forward-looking measure contributes to a restaurant's success.

9. Participants were provided details about the experimental task (i.e., resource allocation).

10. Participants were assigned forward-looking and contemporaneous goals.

11. Participants were provided the details of their incentive contract, including an example bonus calculation.

12. Participants were shown how to access information throughout the simulation (e.g., prior performance data, incentive contract details, etc.).

13. Participants were asked a series of multiple choice and short answer questions about the experimental task.

14. Participants completed one practice round of the simulation.

15. Participants began the 36-period simulation.

16. Goal commitment questions were asked at the end of periods 12 and 24.

17. At the end of period 36, participants were asked task complexity, demographic information, and task characteristic questions.

18. Participants were informed of their total earnings as restaurant manager.

19. The experiment facilitator paid participants. 
Table 8

Participants' Background
Standard
1. Number of

$\frac{\text { Mean }}{7.90} \quad \frac{\text { Median }}{5.00} \quad \frac{\begin{array}{c}\text { Standard } \\ \text { Dev. }\end{array}}{6.02} \quad \frac{\text { Minimum }}{0.00} \frac{\text { Maximum }}{28.00} \quad \frac{\mathrm{N}}{264}$
Accounting Courses

\section{Standard}
2. Participant Earnings $\quad \frac{\text { Mean }}{18.70} \quad \frac{\underline{\text { Median }}}{19.34} \quad \frac{\underline{\text { Dev. }}}{2.85} \quad \frac{\text { Minimum }}{10.80} \frac{\text { Maximum }_{21.60}}{264}$

3. Program of Study:

\begin{tabular}{|c|c|c|}
\hline & Number & Percentage \\
\hline Accounting & 154 & $58 \%$ \\
\hline Mathematics & 76 & $29 \%$ \\
\hline Other & 34 & $13 \%$ \\
\hline Total & 264 & $100 \%$ \\
\hline
\end{tabular}

4. Year in Program:

\begin{tabular}{|c|c|c|}
\hline & Number & Percentage \\
\hline First year & 2 & $1 \%$ \\
\hline Second year & 17 & $6 \%$ \\
\hline Third year & 160 & $61 \%$ \\
\hline Fourth year & 85 & $32 \%$ \\
\hline Total & 264 & $100 \%$ \\
\hline
\end{tabular}

5. Restaurant Work Experience:

\begin{tabular}{lccc} 
& Number & & Percentage \\
No experience & 214 & & $81 \%$ \\
Experience & 50 & & $19 \%$ \\
\cline { 2 - 3 } Total & 264 & $100 \%$ \\
\hline
\end{tabular}

\begin{tabular}{|c|c|c|c|c|c|c|}
\hline & & & Standar & & & \\
\hline & Mean & $\underline{\text { Median }}$ & Dev. & $\underline{\text { Minimum }}$ & Maximum & $\underline{N}$ \\
\hline $\begin{array}{l}\text { 6. Experience in } \\
\text { Restaurant Business } \\
\text { (years): }\end{array}$ & 1.15 & 1.00 & 1.19 & 0.00 & 6.00 & $\overline{50}$ \\
\hline
\end{tabular}


Table 9

Task Complexity Manipulation Check - Questions asked at the end of Period 12 and 24

Panel A: Descriptive Statistics of Complexity Questions Asked in Periods 12 and $24(\mathrm{n}=94)$

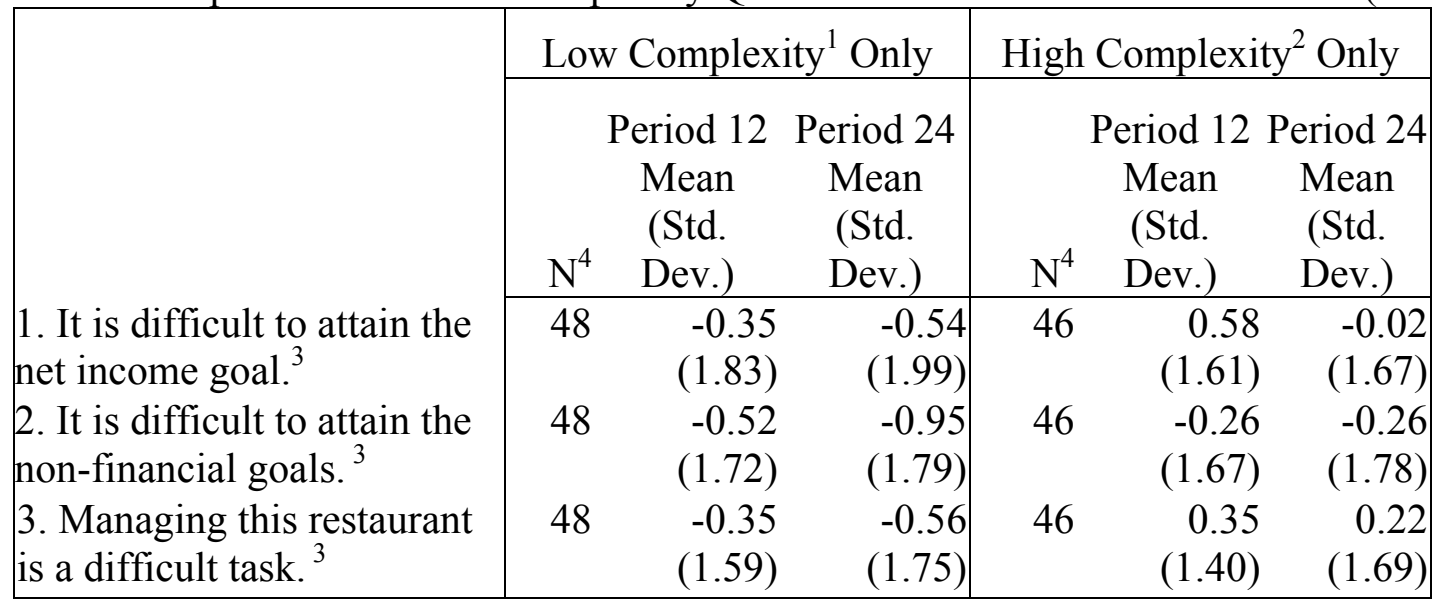

Panel B: Correlations of Complexity Questions Asked in Periods 12 and $24(n=94)$

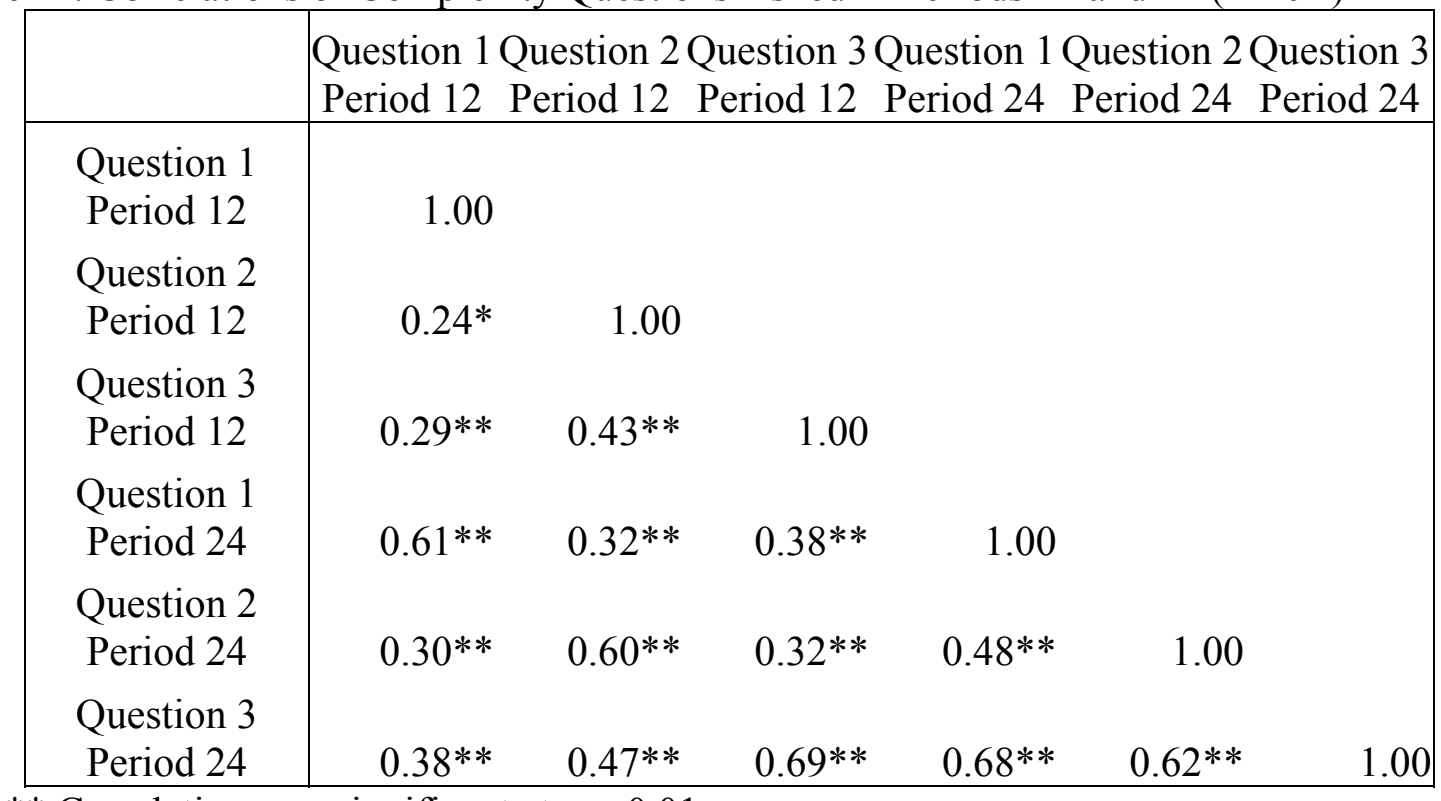

$* *$ Correlations are significant at $\mathrm{p}<0.01$

* Correlations are significant at $\mathrm{p}<0.05$ 
Table 9 (continued)

Panel C: Repeated Measures, Multivariate Analysis of Variance: Three Complexity Questions $(\mathrm{n}=94)$

\begin{tabular}{|l|l|ccc|}
\hline & & & & \multicolumn{2}{c|}{$\begin{array}{c}\text { p-value } \\
\text { (two } \\
\text { tailed) }\end{array}$} \\
\hline Between Subjects & Complexity & 2.58 & 3.00 & $\mathbf{0 . 0 6}$ \\
& Incentives & 0.05 & 6.00 & 0.59 \\
& Complexity x Incentives & 1.10 & 6.00 & 0.36 \\
\hline Within Subjects & Period & 2.31 & 3.00 & $\mathbf{0 . 0 8}$ \\
& Period x Complexity & 1.33 & 3.00 & 0.27 \\
& Period x Incentives & 0.50 & 6.00 & 0.81 \\
& Period x Complexity x Incentives & 1.92 & 6.00 & $\mathbf{0 . 0 8}$ \\
\hline
\end{tabular}

Panel D: Repeated Measures Analysis of Variance: Net Income Goal $(\mathrm{n}=94)$

\begin{tabular}{|c|c|c|c|c|}
\hline & & $\mathrm{F}$ & $\mathrm{df}$ & $\begin{array}{l}\mathrm{p} \text {-value } \\
\text { (two } \\
\text { tailed) }\end{array}$ \\
\hline \multirow[t]{3}{*}{ Between Subjects } & Complexity & 5.27 & 1.00 & 0.02 \\
\hline & Incentives & 0.61 & 2.00 & 0.51 \\
\hline & Complexity x Incentives & .67 & 2.00 & 0.20 \\
\hline \multirow[t]{4}{*}{ Within Subjects } & Period & 6.34 & 1.00 & 0.01 \\
\hline & Period x Complexity & 1.36 & 1.00 & 0.26 \\
\hline & Period $\mathrm{x}$ Incentives & 1.43 & 2.00 & 0.24 \\
\hline & Period x Complexity x Incentives & 2.09 & 3.00 & 0.13 \\
\hline
\end{tabular}

Panel E: Repeated Measures Analysis of Variance: Non Financial Goal ( $\mathrm{n}=94)$

\begin{tabular}{|c|c|c|c|c|}
\hline & & $\mathrm{F}$ & $\mathrm{df}$ & $\begin{array}{l}\mathrm{p} \text {-value } \\
\text { (two } \\
\text { tailed) }\end{array}$ \\
\hline \multirow[t]{3}{*}{ Between Subjects } & Complexity & 2.06 & 1.00 & 0.15 \\
\hline & Incentives & 0.41 & 2.00 & 0.67 \\
\hline & Complexity x Incentives & 0.11 & 2.00 & 0.90 \\
\hline \multirow[t]{4}{*}{ Within Subjects } & Period & 1.62 & 1.00 & 0.21 \\
\hline & Period x Complexity & 1.87 & 1.00 & 0.18 \\
\hline & Period $\mathrm{x}$ Incentives & 0.15 & 2.00 & 0.86 \\
\hline & Period x Complexity $\mathrm{x}$ Incentives & 0.48 & 3.00 & 0.62 \\
\hline
\end{tabular}


Table 9 (continued)

Panel F: Repeated Measures Analysis of Variance: Overall Task $(n=94)$

\begin{tabular}{|l|l|ccc|}
\hline & & & & \multicolumn{2}{c|}{$\begin{array}{c}\text { p-value } \\
\text { (two } \\
\text { tailed) }\end{array}$} \\
\hline Between Subjects & Complexity & 6.44 & 1.00 & $\mathbf{0 . 0 1}$ \\
& Incentives & 1.72 & 2.00 & 0.19 \\
& Complexity x Incentives & 1.60 & 2.00 & 0.21 \\
\hline \multirow{2}{*}{ Within Subjects } & Period & 1.97 & 1.00 & 0.16 \\
& Period x Complexity & 0.11 & 1.00 & 0.72 \\
& Period x Incentive & 0.02 & 2.00 & 0.98 \\
& Period x Complexity x Incentives & 2.91 & 3.00 & $\mathbf{0 . 0 6}$ \\
\hline
\end{tabular}

1

In the high complexity conditions, participants are assigned four, forward-looking measures to manage, and the effect of an investment on sales is lagged three periods.

2

In the low complexity conditions, participants are assigned two forward-looking measures to manage, and the effect of an investment on future sales is lagged one period.

3

Responses were collected using a 7-point Likert scale ranging from -3 (strongly disagree) to +3 (strongly agree).

4 Complexity questions were added to the simulation for the second administration. Participants in the control condition were not asked these questions. Therefore, only the 94 participants assigned to the high, medium and low contemporaneous weighting incentive conditions responded to these three complexity questions. 
Table 10

Task Complexity Manipulation Check - Questions asked at the end of Period 36

Panel A:

Descriptive Statistics

\begin{tabular}{|c|c|c|c|c|c|c|c|c|c|c|}
\hline \multirow[b]{2}{*}{$\begin{array}{c}\text { Complexity } \\
\text { Questions } \\
\text { (see Table 5) }\end{array}$} & \multicolumn{5}{|c|}{ Low Complexity ${ }^{1}$ Incentive Conditions } & \multicolumn{5}{|c|}{ High Complexity ${ }^{2}$ Incentive Conditions } \\
\hline & $\begin{array}{l}\text { Low } \\
\text { Mean } \\
\text { (Std. } \\
\text { Dev'n) } \\
\end{array}$ & $\begin{array}{l}\text { Medium } \\
\text { Mean } \\
\text { (Std. } \\
\text { Dev'n) } \\
\end{array}$ & $\begin{array}{l}\text { High } \\
\text { Mean } \\
\text { (Std. } \\
\text { Dev'n) } \\
\end{array}$ & $\begin{array}{c}\text { Control } \\
\text { Mean } \\
\text { (Std. } \\
\text { Dev'n) } \\
\end{array}$ & \begin{tabular}{|} 
Average \\
Mean \\
(Std. \\
Dev'n) \\
\end{tabular} & $\begin{array}{l}\text { Low } \\
\text { Mean } \\
(\text { Std. } \\
\text { Dev'n) } \\
\end{array}$ & $\begin{array}{l}\text { Medium } \\
\text { Mean } \\
\text { (Std. } \\
\text { Dev'n) } \\
\end{array}$ & $\begin{array}{l}\text { High } \\
\text { Mean } \\
\text { (Std. } \\
\text { Dev'n) } \\
\end{array}$ & $\begin{array}{c}\text { Control } \\
\text { Mean } \\
\text { (Std. } \\
\text { Dev'n) } \\
\end{array}$ & $\begin{array}{l}\text { Average } \\
\text { Mean } \\
\text { (Std. } \\
\text { Dev'n) } \\
\end{array}$ \\
\hline Question $4^{3}$ & $\begin{array}{r}-0.49 \\
(1.50)\end{array}$ & $\begin{array}{r}-0.15 \\
(1.33)\end{array}$ & $\begin{array}{r}-0.03 \\
(1.47)\end{array}$ & $\begin{array}{c}-1.05 \\
(1.19)\end{array}$ & $\begin{array}{r}-0.45 \\
(1.41)\end{array}$ & $\begin{array}{r}0.17 \\
(1.40)\end{array}$ & $\begin{array}{r}0.11 \\
(1.30)\end{array}$ & $\begin{array}{r}0.84 \\
(1.28)\end{array}$ & $\begin{array}{r}-0.03 \\
(1.40)\end{array}$ & $\begin{array}{r}0.27 \\
(1.36)\end{array}$ \\
\hline Que & $\begin{array}{r}0.03 \\
(1.49)\end{array}$ & $\begin{array}{r}0.09 \\
(1.62)\end{array}$ & $\begin{array}{r}0.27 \\
(0.57)\end{array}$ & $\begin{array}{c}-0.34 \\
(1.14)\end{array}$ & $\begin{array}{r}0.02 \\
(1.46)\end{array}$ & $\begin{array}{r}0.40 \\
(1.24)\end{array}$ & $\begin{array}{r}0.49 \\
(1.34)\end{array}$ & $\begin{array}{r}1.19 \\
(1.60)\end{array}$ & $\begin{array}{r}0.08 \\
(1.38)\end{array}$ & $\begin{array}{r}0.5 \\
(1.49\end{array}$ \\
\hline Que & $\begin{array}{r}0.42 \\
(1.85)\end{array}$ & $\begin{array}{r}0.54 \\
(1.70)\end{array}$ & $\begin{array}{r}1.10 \\
(1.63)\end{array}$ & $\begin{array}{r}0.57 \\
(0.17)\end{array}$ & $\begin{array}{r}0.64 \\
(1.73)\end{array}$ & $\begin{array}{r}1.14 \\
(1.24)\end{array}$ & $\begin{array}{r}0.83 \\
(1.71)\end{array}$ & $\begin{array}{r}1.13 \\
(1.76)\end{array}$ & $\begin{array}{r}1.49 \\
(1.61)\end{array}$ & $\begin{array}{r}1.14 \\
(1.60)\end{array}$ \\
\hline Ques & $\begin{array}{r}0.70 \\
(1.43)\end{array}$ & $\begin{array}{r}0.63 \\
(1.37)\end{array}$ & $\begin{array}{r}1.03 \\
(1.54)\end{array}$ & $\begin{array}{c}-0.31 \\
(1.51)\end{array}$ & $\begin{array}{r}0.48 \\
(1.53)\end{array}$ & $\begin{array}{r}1.08 \\
(1.07)\end{array}$ & $\begin{array}{r}0 . \\
(1.5\end{array}$ & $\begin{array}{r}1.09 \\
(1.25)\end{array}$ & $\begin{array}{r}1.03 \\
(1.44)\end{array}$ & $\begin{array}{r}0.95 \\
(1.29)\end{array}$ \\
\hline Que & $\begin{array}{r}-0.70 \\
(1.61)\end{array}$ & $\begin{array}{r}-0.40 \\
(0.135)\end{array}$ & $\begin{array}{r}-0.50 \\
(0.43)\end{array}$ & $\begin{array}{r}-1.02 \\
(1.32)\end{array}$ & $\begin{array}{c}-0.67 \\
(1.43)\end{array}$ & $\begin{array}{r}0.06 \\
(1.30)\end{array}$ & $\begin{array}{r}-0.37 \\
(1.33)\end{array}$ & $\begin{array}{r}0.59 \\
(1.41)\end{array}$ & $\begin{array}{r}-0.49 \\
(1.56)\end{array}$ & $(1.45)$ \\
\hline Que & $\begin{array}{r}0.94 \\
(1.71)\end{array}$ & $\begin{array}{r}0.65 \\
(1.41)\end{array}$ & $\begin{array}{r}0.14 \\
(0.41)\end{array}$ & $\begin{array}{c}-0.24 \\
(1.44)\end{array}$ & $\begin{array}{r}0.38 \\
(1.53)\end{array}$ & $\begin{array}{r}1.00 \\
(1.15)\end{array}$ & $\begin{array}{r}0.88 \\
(1.63)\end{array}$ & $\begin{array}{r}1.62 \\
(1.54)\end{array}$ & $\begin{array}{r}1.31 \\
(1.19)\end{array}$ & $\begin{array}{r}1.18 \\
(1.42)\end{array}$ \\
\hline Quest & $\begin{array}{r}0.29 \\
(1.69)\end{array}$ & $\begin{array}{r}0.29 \\
(1.49)\end{array}$ & $\begin{array}{r}0.57 \\
(1.65)\end{array}$ & $\begin{array}{c}-0.12 \\
(1.22)\end{array}$ & $\begin{array}{r}0.25 \\
(1.50)\end{array}$ & $\begin{array}{r}1.38 \\
(1.03)\end{array}$ & $\begin{array}{r}1.00 \\
(1.32)\end{array}$ & $\begin{array}{r}1.69 \\
(1.49)\end{array}$ & $\begin{array}{r}1.25 \\
(1.06)\end{array}$ & $\begin{array}{r}1.32 \\
(1.26)\end{array}$ \\
\hline $\mathrm{A}$ & $\begin{array}{r}0.11 \\
(1.02)\end{array}$ & $\begin{array}{r}0.17 \\
(1.05)\end{array}$ & $\begin{array}{r}0.42 \\
(1.11)\end{array}$ & $\begin{array}{c}-0.39 \\
(0.91)\end{array}$ & $\begin{array}{r}0.06 \\
(1.05)\end{array}$ & $\begin{array}{r}0.58 \\
(0.77)\end{array}$ & $\begin{array}{r}0.37 \\
(0.99)\end{array}$ & $\begin{array}{r}1.04 \\
(0.99)\end{array}$ & $\begin{array}{r}0.50 \\
(1.06)\end{array}$ & $\begin{array}{r}0.62 \\
(0.98)\end{array}$ \\
\hline
\end{tabular}


Table 10 (continued)

Panel B: Correlations

\begin{tabular}{|c|c|c|c|c|c|c|c|}
\hline Question & 4 & 5 & 6 & 7 & 8 & 9 & 10 \\
\hline 4 & 1.00 & & & & & & \\
\hline 5 & $0.65 * *$ & 1.00 & & & & & \\
\hline 6 & 0.06 & $0.15^{*}$ & 1.00 & & & & \\
\hline 7 & $0.40 * *$ & $0.42 * *$ & $0.57 * *$ & 1.00 & & & \\
\hline 8 & $0.51 * *$ & $0.62 * *$ & $0.27 * *$ & $0.67 * *$ & 1.00 & & \\
\hline 9 & $0.47 * *$ & $0.53 * *$ & $0.19 *$ & $0.46^{* *}$ & $0.59 * *$ & 1.00 & \\
\hline 10 & $0.54 * *$ & $0.50 * *$ & $0.19 * *$ & $0.31 * *$ & $0.52 * *$ & $0.44 * *$ & 1.00 \\
\hline
\end{tabular}

Panel C: Multivariate Analysis: Partial Sample (Questions 4-10, $\mathrm{n}=126^{5}$ )

\begin{tabular}{|c|c|c|c|}
\hline & $\mathrm{F}$ & df & $\begin{array}{l}\text { p-value } \\
\text { (two } \\
\text { tailed) }\end{array}$ \\
\hline Complexity & 3.79 & 7.00 & $<0.01$ \\
\hline Incentives $^{6}$ & 0.90 & 21.00 & 0.60 \\
\hline Complexity x Incentives & 1.74 & 21.00 & 0.02 \\
\hline
\end{tabular}

Panel D: Multivariate Analysis: Full Sample (Questions 4-8, n=264)

\begin{tabular}{|l|rrr|}
\hline & & & \multicolumn{2}{c|}{$\begin{array}{c}\text { p-value } \\
\text { (two }\end{array}$} \\
& F & df & \multicolumn{2}{c|}{ tailed) } \\
\hline Complexity & 4.94 & 5 & $<\mathbf{0 . 0 1}$ \\
Incentives & 1.79 & 15 & $\mathbf{0 . 0 3}$ \\
Complexity x Incentives & 1.78 & 15 & $\mathbf{0 . 0 3}$ \\
\hline
\end{tabular}


Table 10 (continued)

Panel E: Variance Analysis: By Question

\begin{tabular}{|l|rrr|}
\hline & & & $\begin{array}{r}\text { p-value } \\
\text { two } \\
\text { tailed })\end{array}$ \\
\hline Question 4 $(\mathrm{n}=264)$ & \multicolumn{1}{|c}{. } & df & \\
Complexity & 18.00 & 3.00 & $<\mathbf{0 . 0 1}$ \\
Incentives & 5.01 & 1.00 & $<\mathbf{0 . 0 1}$ \\
Complexity x Incentives & 1.29 & 3.00 & 0.30 \\
\hline Question 5 $(\mathrm{n}=264)$ & & & \\
Complexity & 8.44 & 3.00 & $<\mathbf{0 . 0 1}$ \\
Incentives & 3.83 & 1.00 & $\mathbf{0 . 0 1}$ \\
Complexity x Incentives & 0.56 & 3.00 & 0.64 \\
\hline Question 6 $(\mathrm{n}=264)$ & & & \\
Complexity & 5.80 & 3.00 & $\mathbf{0 . 0 2}$ \\
Incentives & 1.20 & 1.00 & 0.31 \\
Complexity x Incentives & 1.07 & 3.00 & 0.36 \\
\hline Question 7 $(\mathrm{n}=264)$ & & & \\
Complexity & 2.75 & 3.00 & $\mathbf{0 . 0 4}$ \\
Incentives & 3.32 & 1.00 & $\mathbf{0 . 0 2}$ \\
Complexity x Incentives & 4.20 & 3.00 & $<\mathbf{0 . 0 1}$ \\
\hline Question 8 $(\mathrm{n}=264)$ & & & \\
Complexity & 11.92 & 3.00 & $<\mathbf{0 . 0 1}$ \\
Incentives & 3.39 & 1.00 & $\mathbf{0 . 0 2}$ \\
Complexity x Incentives & 1.69 & 3.00 & 0.17 \\
\hline Question 9 $(\mathrm{n}=126)$ & & & \\
Complexity & 9.35 & 3.00 & $<\mathbf{0 . 0 1}$ \\
Incentives & 0.38 & 1.00 & 0.77 \\
Complexity x Incentives & 2.63 & 3.00 & $\mathbf{0 . 0 5}$ \\
\hline Question 10 $(\mathrm{n}=126)$ & & & \\
Complexity & 18.16 & 3.00 & $<\mathbf{0 . 0 1}$ \\
Incentives & 0.96 & 1.00 & 0.42 \\
Complexity x Incentives & 0.32 & 3.00 & 0.81 \\
\hline & & & \\
\hline
\end{tabular}




\section{Table 10 (continued)}

1 In the low complexity conditions, participants were assigned two forward-looking measures to manage, and the effect of an investment on future sales was lagged one period.

2 In the high complexity conditions, participants were assigned four forward-looking measures to manage, and the effect of an investment on sales was lagged three periods.

3 Responses were collected using a 7-point Likert scale ranging from -3 (Very Difficult) to +3 (Very Easy).

4 Responses were collected using a 7-point Likert scale ranging from -3 (Strongly Disagree) to +3 (Strongly Agree).

5 Complexity questions were added to the simulation for the second administration. Therefore, only the 126 participants that participated in the second administration responded to these questions.

6 In this analysis, all four incentive conditions are included (i.e., high, medium and low contemporaneous weighting contracts and the control condition). 
Table 11

Task Complexity Manipulation Check - Non Parametric Tests

\begin{tabular}{|c|c|c|c|c|c|c|}
\hline & \multicolumn{3}{|c|}{ Complexity $^{1}$} & \multicolumn{3}{|c|}{$\begin{array}{l}\text { Incentive } \\
\text { Contract }^{2}\end{array}$} \\
\hline & $\begin{array}{l}\text { Chi- } \\
\text { Square }\end{array}$ & df & $\begin{array}{c}\text { p- } \\
\text { value }\end{array}$ & $\begin{array}{c}\text { Chi- } \\
\text { Square }\end{array}$ & df & $\begin{array}{c}\mathrm{p}- \\
\text { value }\end{array}$ \\
\hline Period 12 Questions $(\mathrm{n}=94)^{3}$ & & & & & & \\
\hline 1. It is difficult to attain the net income goal. & 6.73 & 1.00 & 0.01 & 0.15 & 2.00 & 0.92 \\
\hline 2. It is difficult to attain the non-financial goals. & 0.66 & 1.00 & 0.42 & 1.00 & 2.00 & 0.61 \\
\hline 3. Managing this restaurant is a difficult task. & 4.80 & 1.00 & 0.03 & 3.02 & 2.00 & 0.22 \\
\hline Period 24 Questions $(\mathrm{n}=94)^{3}$ & & & & & & \\
\hline 1. It is difficult to attain the net income goal. & 1.95 & 1.00 & 0.16 & 3.30 & 2.00 & 0.19 \\
\hline 2. It is difficult to attain the non-financial goals. & 3.84 & 1.00 & 0.05 & 0.56 & 2.00 & 0.76 \\
\hline 3. Managing this restaurant is a difficult task. & 4.66 & 1.00 & 0.03 & 2.61 & 2.00 & 0.27 \\
\hline Period 36 Questions $(\mathrm{Q} 1-5, \mathrm{n}=264 ; \mathrm{Q} 6-7, \mathrm{n}=126)^{4}$ & & & & & & \\
\hline $\begin{array}{l}\text { 1. How difficult did you find the task of investing in } \\
\text { customer service, food quality, restaurant atmosphere, } \\
\text { and menu variety? }\end{array}$ & 16.09 & 1.00 & $<0.01$ & 14.48 & 3.00 & 0.01 \\
\hline $\begin{array}{l}\text { 2. How difficult did you find the overall task of } \\
\text { maximizing your bonus payouts? }\end{array}$ & 7.42 & 1.00 & 0.01 & 13.01 & 3.00 & 0.01 \\
\hline $\begin{array}{l}\text { 3. Many times, I had to check something before I } \\
\text { made my investment decisions. }\end{array}$ & 5.64 & 1.00 & 0.02 & 4.68 & 3.00 & 0.20 \\
\hline $\begin{array}{l}\text { 4. I had to think about a lot of different things at the } \\
\text { same time to successfully perform this task. }\end{array}$ & 6.10 & 1.00 & 0.01 & 6.48 & 3.00 & 0.09 \\
\hline 5. The required task was difficult to perform. ${ }^{6}$ & 8.61 & 1.00 & $<0.01$ & 9.65 & 3.00 & 0.02 \\
\hline $\begin{array}{l}\text { 6. It was difficult deciding how much to spend on } \\
\text { each activity in order to achieve the net income goal } \\
\text { each period. } 6\end{array}$ & 17.94 & 1.00 & $<0.01$ & 1.52 & 3.00 & 0.68 \\
\hline $\begin{array}{l}\text { 7. It was difficult to determine the impact of the } \\
\text { resource allocation decision on net income. }\end{array}$ & 11.39 & 1.00 & $<0.01$ & 4.50 & 3.00 & 0.21 \\
\hline
\end{tabular}




\section{Table 11 (continued)}

In this analysis both task complexity conditions, high and low, are included.

In this analysis, all four incentive conditions are included (i.e., high, medium and low contemporaneous weighting contracts and the control condition).

3

These questions were added to the simulation for the second administration, but not included in the control condition. Therefore, only the 94 participants assigned to the high, medium and low contemporaneous weighting incentive conditions responded to these complexity questions. Questions 6 and 7 were added to the simulation for the second administration, and included in all four incentive conditions. Thus, only the 126 participants that participated in the second administration responded to these questions.

5 Responses were collected using a 7-point Likert scale ranging from -3 (Very Difficult) to +3 (Very Easy).

6 Responses were collected using a 7-point Likert scale ranging from -3 (Strongly Disagree) to +3 (Strongly Agree). In a high complexity task, participants are assigned four forward-looking measures to manage, and the effect of an investment on sales is lagged three periods. 
Table 12

Participants' Performance in Three Forward-Looking Measures Sets ${ }^{1}$

Panel A: Repeated Measures Multivariate Analysis, Periods 12 and 24 Complexity Questions, Low Complexity Condition ${ }^{2}\left(\mathrm{n}=48^{3}\right)$

Dependent Variable: Average Complexity Score ${ }^{4}$

\begin{tabular}{|c|c|c|c|c|}
\hline & Independent Variables & $\mathrm{F}$ & df & $\begin{array}{c}\text { p-value } \\
\text { (two } \\
\text { tailed) }\end{array}$ \\
\hline \multirow[t]{2}{*}{ Between Subjects } & Incentives $^{5}$ & 0.25 & 6.00 & 0.56 \\
\hline & Measure Set & 0.60 & 3.00 & 0.86 \\
\hline \multirow[t]{3}{*}{ Within Subjects } & Period & 0.21 & 3.00 & 0.89 \\
\hline & Period $x$ Incentives & 0.13 & 3.00 & 0.95 \\
\hline & Period x Measure Set & 1.44 & 6.00 & 0.21 \\
\hline
\end{tabular}

Panel B: Multivariate Analysis, Period 36 Complexity Questions, Low Complexity Condition $\left(\mathrm{n}=132^{6}\right)$

Dependent Variable: Average Complexity Score ${ }^{7}$

\begin{tabular}{|l|rrr|}
\hline & & & \multicolumn{2}{c|}{$\begin{array}{c}\text { p-value } \\
\text { (two } \\
\text { Independent Variables }\end{array}$} & F & df & tailed) \\
\hline Incentives $^{8}$ & 1.70 & 15 & $\mathbf{0 . 0 5}$ \\
Measure Set $^{\mid}$ & 1.81 & 5 & 0.12 \\
\hline
\end{tabular}

1

In all three possible forward-looking measure conditions, participants allocated resources to customer satisfaction and food quality. Three different investment relationship sets were employed. In Set 1, the best investment strategy was an allocation of 5,000 Lira to customer service and 9,000 Lira to food quality. In Set 2, the best investment strategy was an allocation of 9,000 Lira to customer service and 6,000 Lira to food quality. In Set 3, the best investment strategy was an allocation of 8,000 Lira to customer service and 6,000 Lira to food quality.

2 In the low complexity condition, participants were assigned two forward-looking measures to manage, and the effect of an investment on future sales is lagged one period.

3 These questions were added to the simulation for the second administration, but not included in the control condition. Therefore, only the 48 participants assigned to the low complexity condition in the second administration are considered in this analysis.

4 All three questions from Table 8 are included in this analysis.

5 In this analysis, three incentive conditions are included (i.e., high, medium and low contemporaneous weighting contracts).

$6 \quad$ All participants in the low complexity condition $(n=132)$ were included in this analysis.

7 Questions $1-5$ from Table 9 are included in this analysis.

8 In this analysis, all four incentive conditions are included (i.e., high, medium and low contemporaneous weighting contracts and the control condition). 
Table 13

Comparison of Perceived Case Characteristics

Panel A: Descriptive Statistics $(\mathrm{n}=264)$

\begin{tabular}{|l|r|}
\hline & \multicolumn{1}{|c|}{$\begin{array}{c}\text { Mean } \\
\text { (Std. } \\
\text { Deviation) }\end{array}$} \\
\hline 1. Realism of the simulation & \\
2. Ability to easily understand the task & \\
& $(1.65)$ \\
3. Simulation is interesting & \\
& $(1.17)$ \\
1 & 1.69 \\
& $(1.06)$ \\
\hline
\end{tabular}

Panel B: Correlations $(\mathrm{n}=264)$

\begin{tabular}{|l|ccc|}
\hline & Question 1 & Question 2 & Question 3 \\
\hline Question 1 & 1.00 & & \\
Question 2 & $0.14^{*}$ & 1.00 & \\
Question 3 & $0.33^{* *}$ & $0.40^{* *}$ & 1.00 \\
\hline
\end{tabular}

** Correlation is significant at $\mathrm{p}<0.01$

* Correlation is significant at $\mathrm{p}<0.05$

Panel C: Multivariate Analysis of Variance Case Characteristics $(\mathrm{n}=264)$

\begin{tabular}{|c|c|c|c|c|}
\hline & $\mathrm{F}$ & $\mathrm{df}$ & & $\begin{array}{l}\text { o-value } \\
\text { (two } \\
\text { tailed) }\end{array}$ \\
\hline Complexity $^{2}$ & 0.75 & & 3 & 0.53 \\
\hline Incentives $^{3}$ & 0.64 & & 9 & 0.77 \\
\hline Complexity $x$ Incentives & 1.21 & & 9 & 0.28 \\
\hline Experiment Session & 3.29 & & 3 & 0.02 \\
\hline
\end{tabular}

Panel D: Kruskal Wallis Parametric Test: Simulation Characteristics $(n=264)$

\begin{tabular}{|c|c|c|c|c|c|c|}
\hline & $\begin{array}{c}\text { Chi- } \\
\text { Square }\end{array}$ & mplex & $\begin{array}{l}\text { kity } \\
\text { p-value } \\
\text { (two } \\
\text { tailed) }\end{array}$ & \begin{tabular}{|l}
\multicolumn{1}{|c}{ Incen } \\
Chi- \\
Square
\end{tabular} & ive C & $\begin{array}{l}\text { ontract } \\
\text { p-value } \\
\text { (two } \\
\text { tailed) }\end{array}$ \\
\hline Realism of the simulation & 1.06 & 1.00 & 0.30 & 0.13 & 3.00 & 0.99 \\
\hline Ability to easily understand the task & 2.64 & 1.00 & 0.10 & 5.53 & 3.00 & 0.14 \\
\hline Simulation is interesting & 1.43 & 1.00 & 0.70 & 2.27 & 3.00 & 0.52 \\
\hline
\end{tabular}




\section{Table 13 (continued)}

Responses were collected using a 7-point Likert scale ranging from -3 (Strongly Disagree) to +3 (Strongly Agree).

2

In this analysis, both complexity conditions are included (i.e., high and low)

3 In this analysis, all four incentive conditions are included (i.e., high, medium and low contemporaneous weighting contracts and the control condition). 
Table 14

Analysis of Participants' Perceptions of Forward-Looking Measures Importance

Panel A: Descriptive Statistics: Low Task Complexity ${ }^{1}$ Conditions

\begin{tabular}{|c|c|c|c|c|c|c|c|c|c|c|}
\hline & \multicolumn{2}{|c|}{$\begin{array}{c}\text { Low } \\
\text { Contemp. } \\
\text { Weight }^{2}\end{array}$} & \multicolumn{2}{|c|}{$\begin{array}{l}\text { Medium } \\
\text { Contemp. } \\
\text { Weight }^{3}\end{array}$} & \multicolumn{2}{|c|}{$\begin{array}{c}\text { High } \\
\text { Contemp. } \\
\text { Weight }^{4}\end{array}$} & \multicolumn{2}{|c|}{$\begin{array}{c}\text { Control } \\
\text { Condition } \\
\end{array}$} & \multicolumn{2}{|c|}{$\begin{array}{l}\text { All Incentive } \\
\text { Conditions }\end{array}$} \\
\hline & $\mathrm{N}$ & $\begin{array}{l}\text { Mean } \\
\text { (Std. } \\
\text { Dev'n) }\end{array}$ & $\mathrm{N}$ & $\begin{array}{l}\text { Mean } \\
\text { (Std. } \\
\text { Dev'n) }\end{array}$ & $\mathrm{N}$ & $\begin{array}{l}\text { Mean } \\
\text { (Std. } \\
\text { Dev'n) }\end{array}$ & $\mathrm{N}$ & $\begin{array}{l}\text { Mean } \\
\text { (Std. } \\
\text { Dev'n) }\end{array}$ & $\mathrm{N}$ & $\begin{array}{l}\text { Mean } \\
\text { (Std. } \\
\text { Dev'n) }\end{array}$ \\
\hline $\begin{array}{l}\text { Customer } \\
\text { Service }^{6}\end{array}$ & 33 & $\begin{array}{c}44.85 \\
(10.71)\end{array}$ & 34 & $\begin{array}{c}47.55 \\
(12.84)\end{array}$ & 30 & $\begin{array}{c}47.90 \\
(10.59)\end{array}$ & 35 & $\begin{array}{c}44.57 \\
(10.87)\end{array}$ & 132 & $\begin{array}{c}46.17 \\
(11.29)\end{array}$ \\
\hline $\begin{array}{c}\text { Food } \\
\text { Quality }\end{array}$ & 33 & $\begin{array}{c}55.15 \\
(10.72)\end{array}$ & 34 & $\begin{array}{c}52.44 \\
(12.85)\end{array}$ & 30 & $\begin{array}{c}52.10 \\
(10.60)\end{array}$ & 35 & $\begin{array}{c}55.43 \\
(10.87)\end{array}$ & 132 & $\begin{array}{c}53.88 \\
(11.29)\end{array}$ \\
\hline
\end{tabular}

Panel B: Descriptive Statistics: High Task Complexity ${ }^{7}$ Conditions

\begin{tabular}{|c|c|c|c|c|c|c|c|c|c|c|}
\hline & \multicolumn{2}{|c|}{$\begin{array}{c}\text { Low } \\
\text { Contemp. } \\
\text { Weight }\end{array}$} & \multicolumn{2}{|c|}{$\begin{array}{c}\text { Medium } \\
\text { Contemp. } \\
\text { Weight }\end{array}$} & \multicolumn{2}{|c|}{$\begin{array}{c}\text { High } \\
\text { Contemp. } \\
\text { Weight } \\
\end{array}$} & \multicolumn{2}{|c|}{$\begin{array}{c}\text { Control } \\
\text { Condition }\end{array}$} & \multicolumn{2}{|c|}{$\begin{array}{c}\text { All Incentive } \\
\text { Conditions }\end{array}$} \\
\hline & $\mathrm{N}$ & $\begin{array}{l}\text { Mean } \\
\text { (Std. } \\
\text { Dev.) }\end{array}$ & $\mathrm{N}$ & $\begin{array}{l}\text { Mean } \\
\text { (Std. } \\
\text { Dev.) }\end{array}$ & $\mathrm{N}$ & $\begin{array}{l}\text { Mean } \\
\text { (Std. } \\
\text { Dev.) }\end{array}$ & $\mathrm{N}$ & $\begin{array}{l}\text { Mean } \\
\text { (Std. } \\
\text { Dev.) }\end{array}$ & $\mathrm{N}$ & $\begin{array}{l}\text { Mean } \\
\text { (Std. } \\
\text { Dev.) }\end{array}$ \\
\hline $\begin{array}{l}\text { Customer } \\
\text { Service }^{6}\end{array}$ & 32 & $\begin{array}{l}28.97 \\
(7.61)\end{array}$ & 34 & $\begin{array}{l}30.27 \\
(7.09)\end{array}$ & 32 & $\begin{array}{l}30.34 \\
(5.84)\end{array}$ & 34 & $\begin{array}{l}28.52 \\
(5.29)\end{array}$ & 132 & $\begin{array}{l}29.52 \\
(6.49)\end{array}$ \\
\hline $\begin{array}{c}\text { Food } \\
\text { Quality }\end{array}$ & 32 & $\begin{array}{l}32.53 \\
(6.26)\end{array}$ & 34 & $\begin{array}{l}32.02 \\
(7.38)\end{array}$ & 32 & $\begin{array}{l}31.31 \\
(5.95)\end{array}$ & 34 & $\begin{array}{l}33.72 \\
(6.32)\end{array}$ & 132 & $\begin{array}{l}32.41 \\
(6.49)\end{array}$ \\
\hline $\begin{array}{l}\text { Restaurant } \\
\text { Atmoshere }^{6}\end{array}$ & 32 & $\begin{array}{l}20.93 \\
(6.42)\end{array}$ & 34 & $\begin{array}{l}19.95 \\
(6.23)\end{array}$ & 32 & $\begin{array}{l}19.96 \\
(4.36)\end{array}$ & 34 & $\begin{array}{l}21.14 \\
(5.91)\end{array}$ & 132 & $\begin{array}{l}20.50 \\
(5.76)\end{array}$ \\
\hline $\begin{array}{c}\text { Menu } \\
\text { Variety }^{6}\end{array}$ & 32 & $\begin{array}{r}17.56 \\
(5.28) \\
\end{array}$ & 34 & $\begin{array}{l}17.75 \\
(7.25)\end{array}$ & 32 & $\begin{array}{r}18.38 \\
(5.83) \\
\end{array}$ & 34 & $\begin{array}{l}16.60 \\
(6.04) \\
\end{array}$ & 132 & $\begin{array}{l}17.56 \\
(6.12)\end{array}$ \\
\hline
\end{tabular}


Table 14 (continued)

Panel C: Multivariate Analyses of Forward-Looking Investment Perceptions by Incentive Type

\begin{tabular}{|l|ccc|}
\hline & $\mathrm{F}$ & $\mathrm{df}$ & $\begin{array}{c}\text { p-value (two } \\
\text { tailed) }\end{array}$ \\
\hline $\begin{array}{l}\text { Low Task Complexity } \\
\text { Incentive Condition }\end{array}$ & 0.79 & 3.00 & 0.51 \\
$\begin{array}{l}\text { High Task Complexity } \\
\text { Incentive Condition }\end{array}$ & 0.57 & 9.00 & 0.83 \\
\hline
\end{tabular}

Panel D: Kruskal Wallis of Forward-Looking Investment Perceptions by Incentive Type

\begin{tabular}{|l|ccc|}
\hline & $\begin{array}{c}\text { Chi- } \\
\text { Square }\end{array}$ & Df & $\begin{array}{c}\mathrm{p} \\
\text { (two-tailed) }\end{array}$ \\
\hline Low Task Complexity & & & \\
Customer Satisfaction & 2.46 & 3.00 & 0.48 \\
Food Quality & 2.46 & 3.00 & 0.48 \\
& & & \\
High Task Complexity & & & \\
Customer Satisfaction & 3.32 & 3.00 & 0.35 \\
Food Quality & 2.54 & 3.00 & 0.47 \\
Restaurant Atmosphere & 1.78 & 3.00 & 0.62 \\
Menu Variety & 1.63 & 3.00 & 0.65 \\
\hline
\end{tabular}




\section{Table 14 (continued)}

2 and the effect of an investment on future sales is lagged one period.

2 When participants earn the maximum bonus available, forward-looking goal attainment is rewarded with $2 / 3$ of the total incentives available while contemporaneous goal attainment is rewarded with $1 / 3$ of the total incentive available. (Figure 1, Conditions A and D)

3 When participants earn the maximum bonus available, forward-looking goal attainment is rewarded with, reward $1 / 3$ of total incentives available while contemporaneous goal attainment is rewarded with $2 / 3$ of the total incentives available. (Figure 1, Conditions B and E) When participants earn the maximum bonus available, contemporaneous goal attainment is rewarded with all of the incentives available. (Figure 1, Conditions $\mathrm{C}$ and $\mathrm{F}$ )

5

6 In the control condition, participants were paid of flat wage of 600 Lira per period.

Prior to commencing the simulation, participants allocated $100 \%$ to the forward-looking measures they were responsible for in their simulations to reflect their perceptions of how forward-looking measure investments affect future contemporaneous results.

7

manage, and the effect of an investment on sales is lagged three periods. 
Table 15

Reliability and Dimensionality of the Goal Commitment Scale

Panel A: Intercorrelations Among Individual Goal Commitment Items ${ }^{1}(\mathrm{n}=196)^{2}$

Contemporaneous Goal Commitment Period 12

\begin{tabular}{|l|rrrrr|}
\hline & Item 1 & Item 2 & Item 3 & Item 4 & Item 5 \\
\hline Item $1^{3}$ & 1 & & & & \\
${\text { Item } 2^{3}}^{3}$ & 0.45 & 1 & & & \\
${\text { Item } 3^{3}}^{\text {Item } 4^{3}}$ & 0.21 & 0.58 & 1 & & \\
${\text { Item } 5^{3}}^{3}$ & 0.45 & 0.62 & 0.43 & 1 & \\
\hline
\end{tabular}

All correlations are significant at $\mathrm{p}<0.01$

Forward-looking Goal Commitment Period 12

\begin{tabular}{|l|rrrrr|}
\hline & \multicolumn{1}{|c}{ Item 1 } & Item 2 & Item 3 & Item 4 & Item 5 \\
\hline Item 1 & 1 & & & & \\
Item 2 & 0.65 & 1 & & & \\
Item 3 & 0.62 & 0.69 & 1 & & \\
Item 4 & 0.51 & 0.61 & 0.63 & 1 & \\
Item 5 & 0.33 & 0.51 & 0.52 & 0.44 & 1 \\
\hline
\end{tabular}

All correlations are significant at $\mathrm{p}<0.01$

Contemporaneous Goal Commitment Period 24

\begin{tabular}{|l|rrrrr|}
\hline & Item 1 & Item 2 & Item 3 & Item 4 & Item 5 \\
\hline Item 1 & 1 & & & & \\
Item 2 & 0.58 & 1 & & & \\
Item 3 & 0.45 & 0.72 & 1 & & \\
Item 4 & 0.35 & 0.59 & 0.54 & 1 & \\
Item 5 & 0.45 & 0.41 & 0.51 & 0.41 & 1 \\
\hline
\end{tabular}

All correlations are significant at $\mathrm{p}<0.01$

Forward-looking Goal Commitment Period 24

\begin{tabular}{|l|rrrrr|}
\hline & \multicolumn{1}{|r}{ Item 1 } & Item 2 & Item 3 & Item 4 & Item 5 \\
\hline Item 1 & 1 & & & & \\
Item 2 & 0.7 & 1 & & & \\
Item 3 & 0.49 & 0.61 & 1 & & \\
Item 4 & 0.55 & 0.71 & 0.67 & 1 & \\
Item 5 & 0.49 & 0.58 & 0.71 & 0.63 & 1 \\
\hline
\end{tabular}

All correlations are significant at $\mathrm{p}<0.01$ 
Table 15 (continued)

\begin{tabular}{|c|c|c|c|c|}
\hline \multicolumn{5}{|c|}{ Panel B: Analysis of Goal Commitment Measure Reliability $(\mathrm{n}=196)$} \\
\hline & $\begin{array}{c}\text { Forward } \\
\text { Looking } \\
\text { Measures } \\
\text { Period } 12 \\
\end{array}$ & $\begin{array}{c}\text { Forward } \\
\text { Looking } \\
\text { Measures } \\
\text { Period 24 }\end{array}$ & $\begin{array}{l}\text { Contemp. } \\
\text { Measures } \\
\text { Period } 12\end{array}$ & \begin{tabular}{|l} 
Contemp \\
Measures \\
Period 24 \\
\end{tabular} \\
\hline \multicolumn{5}{|l|}{ Factor Loadings ${ }^{4}$ : } \\
\hline Item 1 & 0.78 & 0.77 & 0.66 & 0.72 \\
\hline Item 2 & 0.87 & 0.87 & 0.84 & 0.86 \\
\hline Item 3 & 0.87 & 0.84 & 0.73 & 0.84 \\
\hline Item 4 & 0.80 & 0.86 & 0.79 & 0.75 \\
\hline Item 5 & 0.68 & 0.82 & 0.66 & 0.68 \\
\hline Eigenvalue $^{5}$ & 3.24 & 3.47 & 2.72 & 3.04 \\
\hline Variance Explained ${ }^{6}$ & 0.65 & 0.69 & 0.54 & 0.61 \\
\hline Cronbach's Alpha $^{7}$ & 0.86 & 0.89 & 0.83 & 0.83 \\
\hline
\end{tabular}

Goal commitment was measured using the five-question goal commitment scale, developed by Klein et al. (2001).

Participants in the control conditions were not asked goal commitment questions, leaving a sample of 196 observations.

Item definitions:

Item 1: It's hard to take this goal seriously. (Reverse coding required.)

Item 2: Quite frankly, I don't care if I achieve this goal or not. (Reverse coding required.)

Item 3: I am strongly committed to pursuing this goal.

Item 4: It wouldn't take much to make me abandon this goal. (Reverse coding required.)

Item 5: I think this goal is a good target to strive for

4 A high factor loading (i.e., greater than 0.60) suggests that a high portion of variance in each item is explained by the commitment factor (Stevens 1996).

5 An Eignevalue greater than 1.0 suggests that a strong relationship between the commitment factor and the original items exists (Diekhoff 1992).

6 A variance explained value greater than 0.50 suggests a good factor solution (Diekhoff 1992).

7 A Cronbach's Alpha value greater than 0.70 suggests reliability of the instrument (Diekhoff 1992). 
Table 16

Analysis of Goal Commitment Question Order

Panel A: Descriptive Statistics: Goal Commitment by Question Order ${ }^{1}$

\begin{tabular}{|c|c|c|c|}
\hline & $\begin{array}{l}\text { Forward-looking } \\
\text { Questions First } \\
\text { Mean (Std. } \\
\text { Dev'n) }\end{array}$ & $\begin{array}{l}\text { Contemporaneous. } \\
\text { Questions First } \\
\text { Mean (Std. Dev'n) }\end{array}$ & $\begin{array}{c}\text { All Conditions } \\
\text { Mean (Std. Dev'n) }\end{array}$ \\
\hline & $(n=99)$ & $(n=97)$ & $\left(n=196^{2}\right)$ \\
\hline $\begin{array}{l}\text { Average Forward-looking Goal } \\
\text { Commitment, Period } 12^{3}\end{array}$ & $1.29(1.09)$ & $1.48(1.09)$ & $1.38(1.09)$ \\
\hline $\begin{array}{l}\text { Average Forward-looking Goal } \\
\text { Commitment, Period } 24\end{array}$ & $1.13(1.27)$ & $1.23(1.17)$ & $1.78(1.22)$ \\
\hline $\begin{array}{l}\text { Average Forward-looking Goal } \\
\text { Commitment, Periods } 12 \text { and } 24\end{array}$ & $1.21(1.10)$ & $1.35(1.05)$ & $1.28(1.08)$ \\
\hline $\begin{array}{l}\text { Average Contemporaneous Goal } \\
\text { Commitment, Period } 12^{4}\end{array}$ & $1.39(0.97)$ & $1.03(1.17)$ & $1.21(1.09)$ \\
\hline $\begin{array}{l}\text { Average Contemporaneous Goal } \\
\text { Commitment, Period } 24\end{array}$ & $1.39(1.00)$ & $1.14(1.25)$ & $1.26(1.14)$ \\
\hline $\begin{array}{l}\text { Average Contemporaneous Goal } \\
\text { Commitment, Periods } 12 \text { and } 24\end{array}$ & $1.39(0.93)$ & 1.09 (1.09) & $1.23(1.02)$ \\
\hline
\end{tabular}

Panel B: Repeated Measures, Goal Commitment Scores $(n=196)$

\begin{tabular}{|c|c|c|c|}
\hline Effect & df & $\mathrm{F}$ & $\begin{array}{c}\text { p-value } \\
\text { (two- } \\
\text { tailed) }\end{array}$ \\
\hline \multicolumn{4}{|l|}{ Within Subjects Analysis: } \\
\hline Period & 174.00 & 0.02 & 0.98 \\
\hline Period $\mathrm{x}$ Incentives ${ }^{5}$ & 350.00 & 2.96 & 0.02 \\
\hline Period x Complexity ${ }^{6}$ & 174.00 & 0.42 & 0.66 \\
\hline Period x Question Order ${ }^{7}$ & 174.00 & 0.31 & 0.74 \\
\hline Period x Incentive x Complexity & 350.00 & 2.40 & 0.05 \\
\hline Period x Incentive x Question Order & 350.00 & 2.00 & 0.10 \\
\hline Period x Complexity x Question Order & 174.00 & 0.39 & 0.68 \\
\hline Period x Incentive x Complexity x Question Order & 350.00 & 1.60 & 0.17 \\
\hline \multicolumn{4}{|l|}{ Between Subjects Analysis: } \\
\hline Incentives & 350.00 & 3.81 & $<\mathbf{0 . 0 1}$ \\
\hline Complexity & 174.00 & 6.15 & $<\mathbf{0 . 0 1}$ \\
\hline Question Order & 174.00 & 2.70 & $\mathbf{0 . 0 7}$ \\
\hline Incentive $\mathrm{x}$ Complexity & 350.00 & 0.11 & 0.98 \\
\hline Incentive x Question Order & 350.00 & 0.57 & 0.68 \\
\hline Complexity x Question Order & 174.00 & 0.89 & 0.42 \\
\hline Incentive x Complexity x Question Order & 350.00 & 0.74 & 0.57 \\
\hline
\end{tabular}


Table 16 (continued)

Panel C: Post Hoc Analysis

\begin{tabular}{|c|c|c|c|}
\hline Question Order & $\begin{array}{c}\text { Mean } \\
\text { Difference }\end{array}$ & $\begin{array}{l}\text { Standard } \\
\text { Error }\end{array}$ & $\begin{array}{c}\mathrm{p} \text {-value } \\
\text { (two } \\
\text { tailed, } \\
\text { adjusted }^{8} \text { ) }\end{array}$ \\
\hline $\begin{array}{l}\text { Contemp, Forward-Looking - Forward Looking, Contemp. } \\
\text { Forward-Looking Goal Commitment }\end{array}$ & 0.08 & 0.15 & 0.63 \\
\hline Contemporaneous Goal Commitment & -0.27 & 0.14 & 0.05 \\
\hline
\end{tabular}

1 Two different question orders were used in this study. Forward-looking goal commitment questions for questions were asked either before or after contemporaneous goal commitment questions.

2 Participants in the control conditions were not asked goal commitment questions, leaving a sample of 196 observations.

3 Average forward-looking goal commitment is measured using the average of the five-question goal commitment scale, developed by Klein et al. (2001). Average contemporaneous goal commitment is measured using the average of the five-question goal commitment scale, developed by Klein et al. (2001).

5 Three incentive conditions are included in this analysis (i.e., low, medium and high weight on the contemporaneous measure).

Two complexity conditions are included in this analysis (i.e., low and high complexity)

7 Two different question orders are included in this analysis (i.e., forward-looking goal commitment asked before, or after, contemporaneous goal commitment questions).

Post hoc statistics are corrected using the Bonferroni correction factor. 
Table 17

Analysis of Goal Commitment Means by Incentive and Complexity Conditions

Panel A: Descriptive Statistics: Goal Commitment

\begin{tabular}{|c|c|c|c|c|c|c|c|c|}
\hline \multirow[b]{4}{*}{$\begin{array}{c}\text { Contemporaneous } \\
\text { Weighting }\end{array}$} & \multicolumn{4}{|c|}{ Low Complexity $^{1}(\mathrm{n}=98)$} & \multicolumn{4}{|c|}{ High Complexity $^{2}(\mathrm{n}=97)$} \\
\hline & \multicolumn{2}{|c|}{ Period 12} & \multicolumn{2}{|c|}{ Period 24} & \multicolumn{2}{|c|}{ Period 12} & \multicolumn{2}{|c|}{ Period 24} \\
\hline & \multicolumn{2}{|c|}{$\begin{array}{l}\text { Forward } \\
\text { Looking Contemp. }\end{array}$} & \multicolumn{2}{|c|}{$\begin{array}{l}\text { Forward } \\
\text { Looking Contemp }\end{array}$} & \multicolumn{2}{|c|}{$\begin{array}{l}\text { Forward } \\
\text { Looking Contemp. }\end{array}$} & \multicolumn{2}{|c|}{$\begin{array}{l}\text { Forward } \\
\text { Looking Contemp. }\end{array}$} \\
\hline & $\begin{array}{l}\text { Mean } \\
\text { (Std. } \\
\text { Dev'n) }\end{array}$ & $\begin{array}{l}\text { Mean } \\
\text { (Std. } \\
\text { Dev'n) }\end{array}$ & $\begin{array}{l}\text { Mean }^{3} \\
\text { (Std. } \\
\text { Dev'n) }\end{array}$ & $\begin{array}{l}\text { Mean }^{3} \\
\text { (Std. } \\
\text { Dev'n) }\end{array}$ & $\begin{array}{l}\text { Mean } \\
\text { (Std. } \\
\text { Dev'n) }\end{array}$ & $\begin{array}{l}\text { Mean } \\
\text { (Std. } \\
\text { Dev'n) }\end{array}$ & $\begin{array}{l}\text { Mean } \\
\text { (Std. } \\
\text { Dev'n) }\end{array}$ & $\begin{array}{c}\text { Mean } \\
\text { (Std. } \\
\text { Dev'n) }\end{array}$ \\
\hline & $\begin{array}{r}1.67 \\
(0.87)\end{array}$ & $\begin{array}{r}1.07 \\
(1.18)\end{array}$ & $\begin{array}{r}1.47 \\
(1.06)\end{array}$ & $\begin{array}{r}1.09 \\
(1.31)\end{array}$ & $\begin{array}{r}1.55 \\
(0.89)\end{array}$ & $\begin{array}{r}0.90 \\
(1.18)\end{array}$ & $\begin{array}{r}1.18 \\
(1.12)\end{array}$ & $\begin{array}{r}0.97 \\
(1.19)\end{array}$ \\
\hline Med & $\begin{array}{r}1.50 \\
(1.10)\end{array}$ & $\begin{array}{r}1.55 \\
(0.92)\end{array}$ & $\begin{array}{r}1.47 \\
(1.33)\end{array}$ & $\begin{array}{r}1.53 \\
(1.22)\end{array}$ & $\begin{array}{r}1.44 \\
(0.97)\end{array}$ & $\begin{array}{r}0.90 \\
(1.21)\end{array}$ & $\begin{array}{r}1.12 \\
(1.12)\end{array}$ & $\begin{array}{r}1.47 \\
(1.04)\end{array}$ \\
\hline $\operatorname{High}^{6}$ & $\begin{array}{r}1.23 \\
(1.31)\end{array}$ & $\begin{array}{r}1.69 \\
(0.88)\end{array}$ & $\begin{array}{r}1.21 \\
(1.38)\end{array}$ & $\begin{array}{r}1.65 \\
(0.97)\end{array}$ & $\begin{array}{r}0.90 \\
(1.26)\end{array}$ & $\begin{array}{r}1.22 \\
(0.88)\end{array}$ & $\begin{array}{r}0.71 \\
(1.17)\end{array}$ & $\begin{array}{r}0.96 \\
(0.86)\end{array}$ \\
\hline
\end{tabular}

Panel B: Descriptive Statistics: Goal Commitment Difference Scores ${ }^{7}$

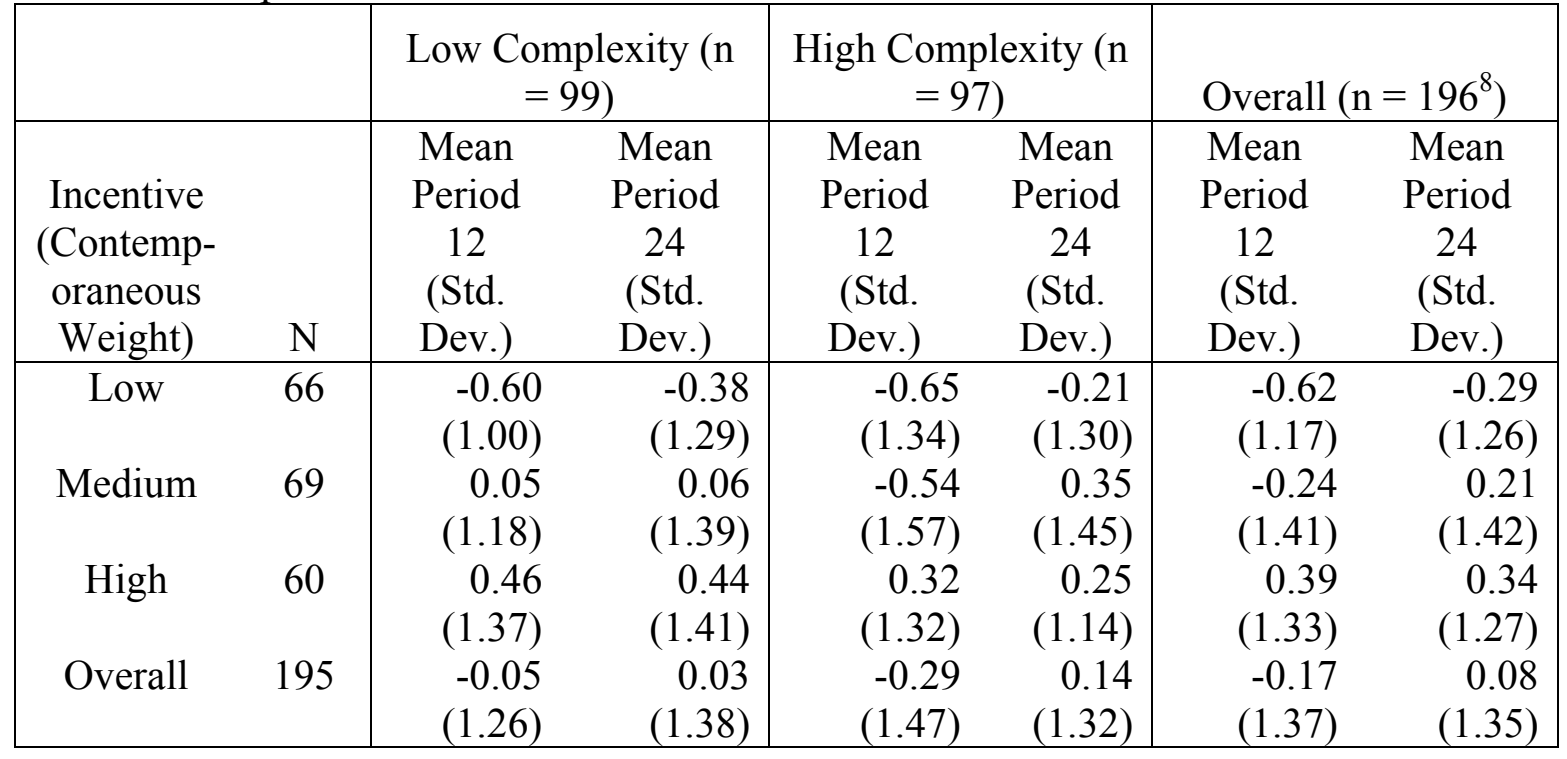


Table 17 (continued)

Panel C: Repeated Measures Analysis of Variance: Goal Commitment Difference Scores Periods 12 and 24

\begin{tabular}{|l|l|llr|}
\hline & & & & \multicolumn{3}{r|}{$\begin{array}{r}\text {-value } \\
\text { (two }\end{array}$} \\
& & F & df & tailed) \\
\hline Between Subjects & Complexity & 0.25 & 1.00 & 0.62 \\
& Incentives & 7.77 & 2.00 & $<\mathbf{0 . 0 1}$ \\
& Complexity x Incentives & 0.18 & 2.00 & 0.83 \\
\hline Within Subjects & Period & 8.32 & 1.00 & $<\mathbf{0 . 0 1}$ \\
& Period x Complexity & 4.07 & 1.00 & $\mathbf{0 . 0 5}$ \\
& Period x Incentives & 3.07 & 2.00 & $\mathbf{0 . 0 5}$ \\
& Period x Complexity x Incentives & 2.60 & 2.00 & $\mathbf{0 . 0 8}$ \\
\hline
\end{tabular}

Panel D: Repeated Measures Analyses of Variance with Period 12 and 24 Goal Commitment Difference Scores as the Dependent Variables

Low Complexity Condition Only

\begin{tabular}{|c|c|c|c|c|}
\hline & & $\mathrm{F}$ & df & $\begin{array}{l}\text { p-value } \\
\text { (two } \\
\text { tailed) }\end{array}$ \\
\hline Between Subjects & Incentives & 5.10 & 2.00 & $<0.01$ \\
\hline \multirow[t]{2}{*}{ Within Subjects } & Period & 0.83 & 1.00 & 0.42 \\
\hline & Period x Incentives & 0.83 & 2.00 & 0.24 \\
\hline
\end{tabular}

High Complexity Condition Only

\begin{tabular}{|c|c|c|c|c|}
\hline & & $\mathrm{F}$ & $\mathrm{df}$ & $\begin{array}{l}\mathrm{p} \text {-value } \\
\text { (two } \\
\text { tailed) }\end{array}$ \\
\hline Between Subject & Incentives & 02.70 & 2.00 & 0.07 \\
\hline \multirow[t]{2}{*}{ Within Subjects } & Period & 8.57 & 1.00 & $<0.01$ \\
\hline & Period x Incentives & 3.87 & 2.00 & 0.02 \\
\hline
\end{tabular}


Table 17 (continued)

Panel E: Repeated Measures Analyses of Variance with Period 12 and 24 Goal Commitment Difference Scores as the Dependent Variables

Low Contemporaneous Weighting Only

\begin{tabular}{|c|c|c|c|c|}
\hline & & $\mathrm{F}$ & df & $\begin{array}{l}\mathrm{p} \text {-value } \\
\text { (two } \\
\text { tailed) }\end{array}$ \\
\hline Between Subjects & Complexity & 0.13 & 1.00 & 0.73 \\
\hline \multirow[t]{2}{*}{ Within Subjects } & Period & 6.75 & 1.00 & 0.01 \\
\hline & Period x Complexity & 0.51 & 1.00 & 0.48 \\
\hline
\end{tabular}

Medium Contemporaneous Weighting Only

\begin{tabular}{|c|c|c|c|c|}
\hline & & $\mathrm{F}$ & df & $\begin{array}{c}\mathrm{p} \text {-value } \\
\text { (two } \\
\text { tailed) }\end{array}$ \\
\hline Between Subjects & Complexity & 0.03 & 1.00 & 0.71 \\
\hline \multirow[t]{2}{*}{ Within Subjects } & Period & 8.02 & 1.00 & 0.01 \\
\hline & Period x Complexity & 5.65 & 1.00 & 0.02 \\
\hline
\end{tabular}

High Contemporaneous Weighting Only

\begin{tabular}{|c|c|c|c|c|}
\hline & & $\mathrm{F}$ & df & $\begin{array}{c}\mathrm{p} \text {-value } \\
\text { (two } \\
\text { tailed) }\end{array}$ \\
\hline Between Subjects & Complexity & 6.13 & 1.00 & 0.02 \\
\hline \multirow[t]{2}{*}{ Within Subjects } & Period & 0.10 & 1.00 & 0.76 \\
\hline & Period x Complexity & 0.03 & 1.00 & 0.86 \\
\hline
\end{tabular}

Panel F: Multiple Comparison Planned Contrasts

\begin{tabular}{|c|r|r|r|c|}
\hline $\begin{array}{c}\text { Goal } \\
\text { Commitment } \\
\text { Score }\end{array}$ & $\begin{array}{c}\text { Sum of } \\
\text { Squares }\end{array}$ & \multicolumn{1}{c|}{ df } & \multicolumn{1}{c|}{ F } & \multicolumn{1}{c|}{$\begin{array}{c}\text { p-value } \\
\text { (two } \\
\text { tailed) }\end{array}$} \\
\hline Period 12 & 32.56 & 1.00 & 18.86 & $<\mathbf{0 . 0 1}$ \\
Period 24 & 12.88 & 1.00 & 7.28 & $<\mathbf{0 . 0 1}$ \\
\hline
\end{tabular}


Table 17 (continued)

Panel G: Multiple Comparison Planned Contrasts

\begin{tabular}{|c|c|c|c|c|c|c|}
\hline & \multicolumn{3}{|c|}{$\begin{array}{c}\text { Goal Commitment Difference } \\
\text { Scores Period } 12\end{array}$} & \multicolumn{3}{|c|}{$\begin{array}{c}\text { Goal Commitment Difference } \\
\text { Scores Period } 24 \\
\end{array}$} \\
\hline Planned Contrast & $\begin{array}{c}\text { Mean } \\
\text { Difference }\end{array}$ & $\begin{array}{l}\text { Standard } \\
\text { Error }\end{array}$ & $\begin{array}{c}\mathrm{p} \text {-value } \\
\text { (one tailed, } \\
\text { adjusted) }\end{array}$ & $\begin{array}{c}\text { Mean } \\
\text { Difference }\end{array}$ & $\begin{array}{l}\text { Standard } \\
\text { Error }\end{array}$ & $\begin{array}{c}\mathrm{p} \text {-value } \\
\text { (one tailed, } \\
\text { adjusted) }\end{array}$ \\
\hline Low - Medium & $(0.44)$ & 0.21 & 0.05 & $(0.54)$ & 0.23 & 0.05 \\
\hline Medium - High & $(0.39)$ & 0.21 & 0.09 & $(0.08)$ & 0.23 & 0.50 \\
\hline Low - High & $(0.82)$ & 0.21 & $<0.01$ & $(0.62)$ & 0.23 & 0.01 \\
\hline
\end{tabular}

\section{Panel H: Non-Parametric Test}

\begin{tabular}{|c|rr|rr|}
\hline & \multicolumn{2}{|c|}{ Period 12 } & \multicolumn{2}{c|}{ Period 24 } \\
\cline { 2 - 5 } & $\begin{array}{r}\text { Mann- } \\
\text { Whitney U }\end{array}$ & $\begin{array}{c}\text { p-value } \\
\text { (one- } \\
\text { tailed) }\end{array}$ & $\begin{array}{c}\text { Mann- } \\
\text { Whitney U }\end{array}$ & $\begin{array}{c}\text { p-value } \\
\text { (one- } \\
\text { tailed) }\end{array}$ \\
\hline Low - Medium & 1788.50 & $\mathbf{0 . 0 3}$ & 1855.00 & $\mathbf{0 . 0 5}$ \\
Medium - High & 1734.50 & $\mathbf{0 . 0 4}$ & 2047.50 & 0.39 \\
Low - High & 1284.50 & $<\mathbf{0 . 0 1}$ & 1617.00 & $\mathbf{0 . 0 3}$ \\
\hline
\end{tabular}

In the low complexity conditions, participants were assigned two forward-looking measures to manage, and the effect of investments on sales was lagged one period.

2

In the high complexity conditions, participants were assigned four forward-looking measures to manage, and the effect of an investment on sales was lagged three periods.

3

Commitment Means were the average of participant responses from the five-question goal commitment scale, developed by Klein et al. (2001). Commitment levels towards the forward-looking and contemporaneous goals were measured separately, at the end of periods 12 and 24.

4 When participants earn the maximum bonus available, forward-looking goal attainment is rewarded with $2 / 3$ of the total incentives available while contemporaneous goal attainment is rewarded with $1 / 3$ of the total incentive available. (Figure 1, Conditions A and D)

5 When participants earn the maximum bonus available, forward-looking goal attainment is rewarded with, reward $1 / 3$ of total incentives available while contemporaneous goal attainment is rewarded with $2 / 3$ of the total incentives available. (Figure 1, Conditions B and E)

6 When participants earn the maximum bonus available, contemporaneous goal attainment is rewarded with all of the incentives available. (Figure 1, Conditions $\mathrm{C}$ and $\mathrm{F}$ )

7 Commitment difference score is the difference between contemporaneous and forward-looking goal commitment, where a positive number indicates higher levels of contemporaneous goal commitment, and a negative number indicates higher levels of forward-looking goal commitment.

8 Sample size is 196 (not the full sample of 264) because the participants in the control conditions were not asked any goal commitment questions. 
Table 18

Analysis of Net Income - Low Complexity Condition

Panel A: Descriptive Statistics: Net Income by Forward-looking Measure, Low Complexity Condition ${ }^{1}$

\begin{tabular}{|c|c|c|c|c|c|}
\hline & $\begin{array}{c}\text { Low } \\
\text { Contemp. } \\
\text { Weight }^{2} \\
\text { Mean } \\
\text { (Std. Dev'n) }\end{array}$ & $\begin{array}{c}\text { Medium } \\
\text { Contemp. } \\
\text { Weight }^{3} \\
\text { Mean } \\
\text { (Std. Dev'n) }\end{array}$ & $\begin{array}{c}\text { High } \\
\text { Contemp. } \\
\text { Weight }^{4} \\
\text { Mean } \\
\text { Std. Dev'n }\end{array}$ & $\begin{array}{c}\text { Control } \\
\text { Condition }^{5} \\
\text { Mean } \\
\text { (Std. Dev'n) }\end{array}$ & $\begin{array}{c}\text { All } \\
\text { Incentive } \\
\text { Conditions } \\
\text { Mean } \\
\text { (Std. Dev'n }\end{array}$ \\
\hline Net Income Periods $2-36^{6}$ & $\begin{array}{c}(n=33) \\
114,247 \\
(40,647)\end{array}$ & $\begin{array}{c}(n=35) \\
125,389 \\
(36,675)\end{array}$ & $\begin{array}{c}(\mathrm{n}=30) \\
129,988 \\
(27,328)\end{array}$ & $\begin{array}{c}(n=35) \\
125,786 \\
(41,675)\end{array}$ & $\begin{array}{l}(n=133) \\
123,766 \\
(37,305)\end{array}$ \\
\hline Net Income Periods 2-12 & $\begin{array}{c}29,792 \\
(14,017)\end{array}$ & $\begin{array}{c}32,737 \\
(13,074)\end{array}$ & $\begin{array}{c}31,178 \\
(16,572)\end{array}$ & $\begin{array}{c}30,207 \\
(14,346)\end{array}$ & $\begin{array}{c}30,989 \\
(14,365)\end{array}$ \\
\hline Net Income Periods 13-24 & $\begin{array}{c}41,524 \\
(15,915)\end{array}$ & $\begin{array}{c}44,630 \\
(14,777)\end{array}$ & $\begin{array}{l}47,484 \\
(8,849)\end{array}$ & $\begin{array}{c}45,873 \\
(14,133)\end{array}$ & $\begin{array}{c}44,830 \\
(13,818)\end{array}$ \\
\hline Net Income Periods 25-36 & $\begin{array}{c}42,932 \\
(13,898)\end{array}$ & $\begin{array}{c}48,023 \\
(12,746)\end{array}$ & $\begin{array}{c}51,327 \\
(10,559)\end{array}$ & $\begin{array}{c}49,706 \\
(16,182)\end{array}$ & $\begin{array}{c}47,948 \\
(13,796)\end{array}$ \\
\hline
\end{tabular}

Panel B: Test for Sphericity: Net Income $(n=98)$

\begin{tabular}{|c|cccc|}
\hline $\begin{array}{c}\text { Within } \\
\text { Subjects } \\
\text { Effect }\end{array}$ & $\begin{array}{c}\text { Mauchly's } \\
\text { W }\end{array}$ & $\begin{array}{c}\text { Approx. } \\
\text { Chi- } \\
\text { Square }\end{array}$ & df & p-value. \\
\hline Period & 0.00 & 2796.17 & 629.00 & $<\mathbf{0 . 0 1}$ \\
\hline
\end{tabular}

Panel C: Repeated Measures, Multivariate Analysis of Net Income ( $\mathrm{n}=98)$

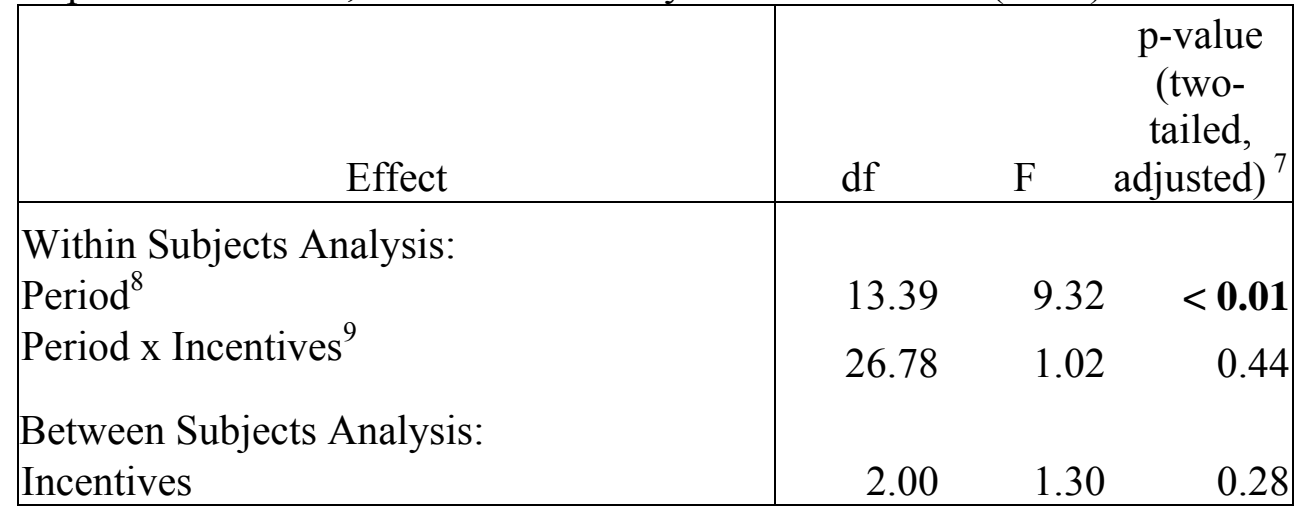


Table 18 (continued)

Panel D: Analyses of Variances: Net Income, by Incentive Condition

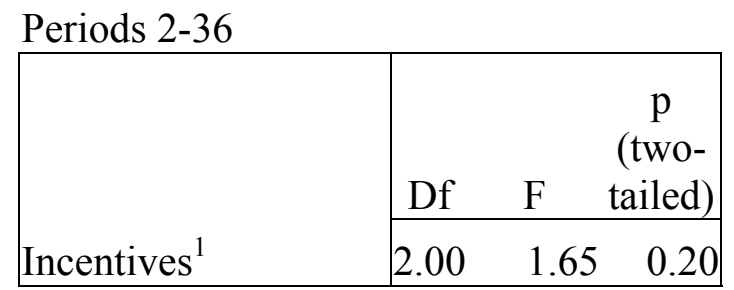

Periods 2-12

\begin{tabular}{|l|ccr|}
\hline \multirow{4}{*}{ Incentives $^{1}$} & & & $\begin{array}{r}\mathrm{p} \\
\text { (two- } \\
\text { tailed) }\end{array}$ \\
\cline { 2 - 4 } & Df & $\mathrm{F}$ & tar \\
\cline { 2 - 4 } & 2.00 & 0.35 & 0.71 \\
\hline
\end{tabular}

Periods $13-24$

\begin{tabular}{|l|ccc|}
\hline \multirow{4}{*}{ Incentives $^{1}$} & & & $\begin{array}{c}\mathrm{p} \\
\text { (two- } \\
\end{array}$ \\
\cline { 2 - 4 } & Df & $\mathrm{F}$ & tailed) \\
\cline { 2 - 4 } & 2.00 & 1.50 & 0.23 \\
\hline
\end{tabular}

Periods 25-36

\begin{tabular}{|c|c|c|c|}
\hline & Df & $\mathrm{F}$ & $\begin{array}{c}\mathrm{p} \\
\text { (two- } \\
\text { tailed) }\end{array}$ \\
\hline Incentives $^{1}$ & 2.00 & 3.61 & $\begin{array}{ll}1 \quad 0.03 \\
\end{array}$ \\
\hline
\end{tabular}


Table 18 (continued)

Panel E: Planned Contrasts

\begin{tabular}{|c|c|c|c|}
\hline & \multicolumn{3}{|c|}{ Average } \\
\hline Net Income & $\begin{array}{c}\text { Mean } \\
\text { Difference }\end{array}$ & $\begin{array}{c}\text { Standard } \\
\text { Error }\end{array}$ & $\begin{array}{c}\text { p-value } \\
\text { (one } \\
\text { tailed, } \\
\text { adjusted }^{10} \text { ) }\end{array}$ \\
\hline \multicolumn{4}{|l|}{ Periods 2 - 36} \\
\hline Low - Medium & $-11,142.09$ & $8,632.95$ & 0.30 \\
\hline Medium - High & $-4,599.04$ & $8,852.33$ & 0.50 \\
\hline Low - High & $-15,741.13$ & $8,975.29$ & 0.12 \\
\hline \multicolumn{4}{|l|}{ Periods 2 - 12} \\
\hline Low - Medium & $-2,945.56$ & $3,526.14$ & 0.50 \\
\hline Medium - High & $1,558.71$ & $3,615.75$ & 0.50 \\
\hline Low - High & $-1,386.86$ & $3,665.97$ & 0.50 \\
\hline \multicolumn{4}{|l|}{ Periods 13 - 24} \\
\hline Low - Medium & $-3,105.63$ & $3,321.45$ & 0.50 \\
\hline Medium - High & $-2,853.85$ & $3,405.85$ & 0.50 \\
\hline Low - High & $-5,959.47$ & $3,453.16$ & 0.13 \\
\hline \multicolumn{4}{|l|}{ Periods $25-36$} \\
\hline Low - Medium & $-5,090.90$ & $3,042.60$ & 0.15 \\
\hline Medium - High & $-3,303.90$ & $3,119.92$ & 0.44 \\
\hline Low - High & $-8,394.80$ & $3,163.25$ & 0.01 \\
\hline
\end{tabular}

Panel F: Kruskal Wallis Test, Comparing the Effect of Incentive Conditions on Net Income

\begin{tabular}{|c|c|c|c|c|c|c|c|c|}
\hline & \multicolumn{2}{|c|}{ Period $1-36$} & \multicolumn{2}{|c|}{ Period 1 - 12} & \multicolumn{2}{|c|}{ Period $13-24$} & \multicolumn{2}{|c|}{ Period $25-36$} \\
\hline & $\begin{array}{c}\text { Chi- } \\
\text { Square }\end{array}$ & $\begin{array}{c}\text { p-value } \\
\text { (two- } \\
\text { tailed) }\end{array}$ & $\begin{array}{l}\text { Chi- } \\
\text { Square }\end{array}$ & $\begin{array}{l}\text { p-value } \\
\text { (two- } \\
\text { tailed) }\end{array}$ & $\begin{array}{l}\text { Chi- } \\
\text { Square }\end{array}$ & $\begin{array}{c}\text { p-value } \\
\text { (two- } \\
\text { tailed) }\end{array}$ & $\begin{array}{l}\text { Chi- } \\
\text { Square }\end{array}$ & $\begin{array}{l}\text { p-value } \\
\text { (two- } \\
\text { tailed) }\end{array}$ \\
\hline Incentives & 3.07 & 0.22 & 1.04 & 0.60 & 2.41 & 0.30 & 7.60 & 0.02 \\
\hline
\end{tabular}


Table 18 (continued)

Panel G: Mann Whitney Test, Comparing the Effect of Incentive Conditions on Net Income

\begin{tabular}{|c|cc|cc|cc|cc|}
\hline & \multicolumn{2}{|c|}{ Period 1-36 } & \multicolumn{2}{|c|}{ Period 1 - 12 } & \multicolumn{2}{c|}{ Period 13 - 24 } & \multicolumn{2}{c|}{ Period 25 - 36 } \\
\cline { 2 - 9 } & $\begin{array}{c}\text { Mann- } \\
\text { p-value } \\
\text { Whitney } \\
\text { (one- } \\
\text { Incentives }\end{array}$ & $\begin{array}{c}\text { Mann- } \\
\text { tailed) }\end{array}$ & $\begin{array}{c}\text { Whitney } \\
\text { U }\end{array}$ & $\begin{array}{c}\text { (one- } \\
\text { tailed) }\end{array}$ & $\begin{array}{c}\text { Mann- } \\
\text { Whitney } \\
\text { U }\end{array}$ & $\begin{array}{c}\text { p-value } \\
\text { (one- } \\
\text { tailed) }\end{array}$ & $\begin{array}{c}\text { Mann- } \\
\text { Whitney } \\
\text { U }\end{array}$ & $\begin{array}{c}\text { (one- } \\
\text { tailed) }\end{array}$ \\
\hline Low - Medium & 450.00 & 0.06 & 520.50 & 0.24 & 482.50 & 0.12 & 409.00 & $\mathbf{0 . 0 2}$ \\
Medium - High & 514.00 & 0.44 & 512.50 & 0.43 & 498.00 & 0.37 & 447.50 & 0.15 \\
Low - High & 389.50 & 0.07 & 420.00 & 0.15 & 389.00 & $\mathbf{0 . 0 7}$ & 317.00 & $<\mathbf{0 . 0 1}$ \\
\hline
\end{tabular}

1 In the low complexity conditions, participants were assigned two forward-looking measures to manage, and the effect of the investment on future sales were lagged one period.

2 When participants earn the maximum bonus available, forward-looking goal attainment is rewarded with $2 / 3$ of the total incentives available while contemporaneous goal attainment is rewarded with $1 / 3$ of the total incentive available. (Figure 1, Conditions A and D)

3 When participants earn the maximum bonus available, forward-looking goal attainment is rewarded with, reward $1 / 3$ of total incentives available while contemporaneous goal attainment is rewarded with $2 / 3$ of the total incentives available. (Figure 1, Conditions B and E)

4 When participants earn the maximum bonus available, contemporaneous goal attainment is rewarded with all of the incentives available. (Figure 1, Conditions $\mathrm{C}$ and F)

5 In the control condition, participants were paid of flat wage of 600 Lira per period.

6 Due to the lagged effect of investments on net income one period, the first period of operations does not reflect the quality of participants' decisions, and as a result, has been excluded from this analysis.

7 Due to the violation of the sphericity assumption, the p-values are adjusted using the Huynh and Feldt correction factor (Keppel 1973).

8 Thirty-five simulation periods are included in this analysis.

9 Three incentive contracts are included in this analysis (i.e., incentives with low, medium and high weightings on contemporaneous measure.)

10 Planned contrast statistics are corrected using the Bonferroni correction factor. 
Table 19

Mediation Analysis - Low Complexity Condition ${ }^{1}$

Panel A: Correlations $(\mathrm{n}=98)$

\begin{tabular}{|c|c|c|c|c|}
\hline & $\begin{array}{c}\text { Commitmen } \\
\text { Difference } \\
\text { Score, } \\
\text { Period } 12 \\
\end{array}$ & $\begin{array}{l}\text { Commitment } \\
\text { Difference } \\
\text { Score, } \\
\text { Period } 24 \\
\end{array}$ & $\begin{array}{c}\text { Net Income, } \\
\text { Periods } \\
13-24 \\
\end{array}$ & $\begin{array}{c}\text { Net Income, } \\
\text { Periods } \\
25-36 \\
\end{array}$ \\
\hline Commitment Difference Score ${ }^{2}$, Period 12 & 1.00 & & & \\
\hline Commitment Difference Score, Period 24 & $0.73 * *$ & 1.00 & & \\
\hline Net Income, Periods 13 - 24 & $0.21 * *$ & $0.24 *$ & 1.00 & \\
\hline Net Income, Periods $25-36$ & $0.09 *$ & 0.13 & $0.83 * *$ & 1.00 \\
\hline
\end{tabular}

** Correlation is significant at $\mathrm{p}<0.01$

* Correlation is significant at $\mathrm{p}<0.05$

Panel B: Mediation Analysis Standardized Coefficients (p-value, one-tailed) ( $n=98)$

\begin{tabular}{|c|c|c|c|}
\hline & \multicolumn{3}{|c|}{ Dependent Variables ${ }^{3}$} \\
\hline \multirow{9}{*}{$\begin{array}{l}\text { Incentives }^{4} \\
\text { Commitment Difference Score, Period } 24^{\text {Adjusted } \mathrm{R}^{2}} \\
\text { Mediation Condition Satisfied }\end{array}$} & (1) & (2) & (3) \\
\hline & Cumulative & Commitment & Cumulative \\
\hline & Net Income & Difference & Net Income \\
\hline & Periods & Score & Periods \\
\hline & $25-36$ & Period 24 & $25-36$ \\
\hline & $0.32(<0.01)$ & $0.29(0.01)$ & $0.33(0.01)$ \\
\hline & & & $-0.03(0.42)$ \\
\hline & 0.32 & 0.29 & 0.33 \\
\hline & Yes & Yes & No \\
\hline
\end{tabular}




\section{Table 19 (continued)}

1 In the low complexity conditions, participants were assigned two forward-looking measures to manage, and the effect of the investment on future sales were lagged one period.

2 Commitment difference score is the difference between contemporaneous and forward-looking goal commitment, where a positive number indicates higher levels of contemporaneous goal commitment, and a negative number indicates higher levels of forward-looking goal commitment.

3 To test mediation, I estimate three regressions, as specified by Baron and Kenny (1986). The three regressions are as follows:

1. Cumulative Net Income $=a_{0}+a_{1} *$ Incentive Contract Condition

2. Commitment Difference Score $=b_{0}+b_{1} *$ Incentive Contract Condition

3. Cumulative Net Income $=c_{0}+c_{1} *$ Incentive Contract Condition + $c_{2} *$ Commitment Difference Score

4. The dummy variable for the high contemporaneous weighting incentive contract is equal to 1 if this contract was assigned, and 0 if it was not. 
Table 20

Analysis of Net Income - High Complexity Condition ${ }^{1}$

Panel A: Descriptive Statistics of Net Income

\begin{tabular}{|c|c|c|c|c|c|}
\hline & $\begin{array}{c}\text { Low } \\
\text { Contemp. } \text { Weight } \\
\text { Wean }^{2} \\
\text { Mean } \\
\text { (Std. Dev'n) }\end{array}$ & $\begin{array}{c}\text { Medium } \\
\text { Contemp. } \\
\text { Weight }^{3} \\
\text { Mean } \\
\text { (Std. Dev'n) }\end{array}$ & $\begin{array}{c}\text { High } \\
\text { Contemp. } \\
\text { Weight }^{4} \\
\text { Mean } \\
\text { (Std. Dev'n) }\end{array}$ & $\begin{array}{c}\text { Control } \\
\text { Condition } \\
\text { Mean } \\
\text { (Std. } \\
\text { Dev'n) } \\
\end{array}$ & $\begin{array}{c}\text { All } \\
\text { Incentive } \\
\text { Conditions } \\
\text { Mean } \\
\text { (Std. } \\
\text { Dev'n) } \\
\end{array}$ \\
\hline Net Income Periods $4-36^{6}$ & $\begin{array}{c}(\mathrm{n}=35) \\
96,220 \\
(105,701)\end{array}$ & $\begin{array}{c}(n=35) \\
135,657 \\
(74,948)\end{array}$ & $\begin{array}{c}(\mathrm{n}=32) \\
95,840 \\
(128,756)\end{array}$ & $\begin{array}{c}(\mathrm{n}=37) \\
133,301 \\
(106,450)\end{array}$ & $\begin{array}{c}(\mathrm{n}=139) \\
115,933 \\
(105,776)\end{array}$ \\
\hline Net Income Periods 4-12 & $\begin{array}{c}13,254 \\
(39,458)\end{array}$ & $\begin{array}{c}18,821 \\
(30,968)\end{array}$ & $\begin{array}{c}17,003 \\
(42,619)\end{array}$ & $\begin{array}{c}17,650 \\
(45,185)\end{array}$ & $\begin{array}{c}16,689 \\
(39,559)\end{array}$ \\
\hline Net Income Periods 13-24 & $\begin{array}{c}41,886 \\
(45,512)\end{array}$ & $\begin{array}{c}55,854 \\
(35,163)\end{array}$ & $\begin{array}{c}37,782 \\
(55,449)\end{array}$ & $\begin{array}{c}50,972 \\
(39,540)\end{array}$ & $\begin{array}{c}46,877 \\
(44,316)\end{array}$ \\
\hline Net Income Periods 25-36 & $\begin{array}{c}41,080 \\
(46,042)\end{array}$ & $\begin{array}{c}60,982 \\
(21,398)\end{array}$ & $\begin{array}{c}41,055 \\
(47,673)\end{array}$ & $\begin{array}{c}64,679 \\
(42,425)\end{array}$ & $\begin{array}{c}52,368 \\
(41,673)\end{array}$ \\
\hline
\end{tabular}

Panel B: Test for Sphericity: Net Income

\begin{tabular}{|c|cccc|}
\hline $\begin{array}{c}\text { Within } \\
\text { Subjects } \\
\text { Effect }\end{array}$ & $\begin{array}{c}\text { Mauchly's } \\
\text { W }\end{array}$ & $\begin{array}{c}\text { Approx. } \\
\text { Chi- } \\
\text { Square }\end{array}$ & df & Sig. \\
\hline Period & 0.00 & 2501.18 & 527.00 & $<\mathbf{0 . 0 1}$ \\
\hline
\end{tabular}

Panel C: Repeated Measures, Multivariate Analysis of Net Income

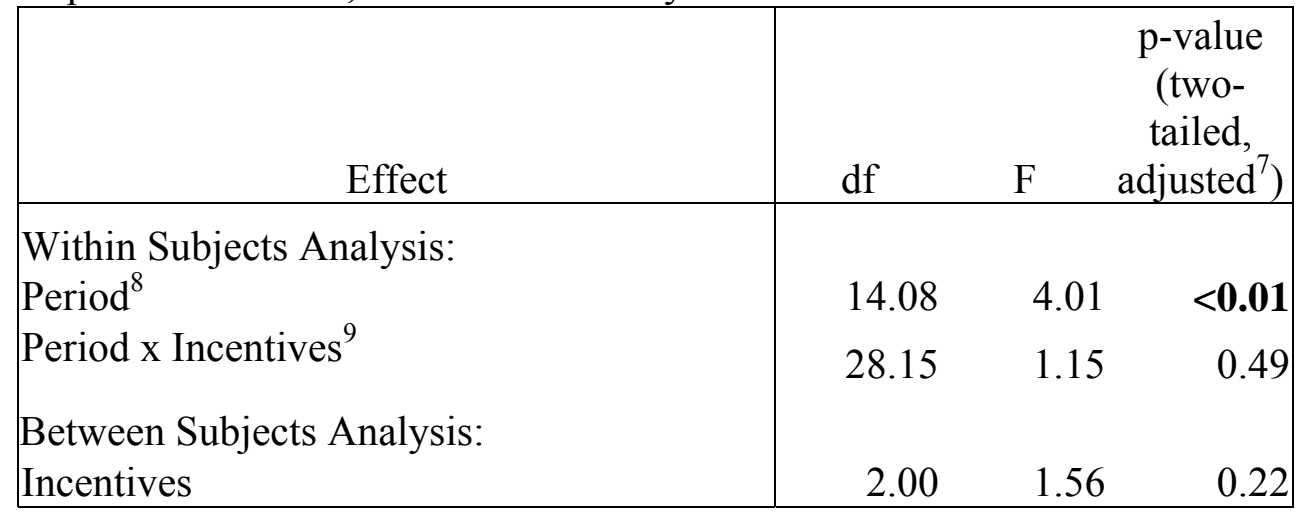


Table 20 (continued)

Panel D: Analyses of Variances: Net Income, by Incentive Condition

Periods 4-36

\begin{tabular}{|c|c|c|c|}
\hline & Df & $\mathrm{F}$ & $\begin{array}{c}\mathrm{p} \\
\text { (two- } \\
\text { tailed) }\end{array}$ \\
\hline ncentives & 2.00 & 1.63 & 0.20 \\
\hline
\end{tabular}

Periods 4-12

\begin{tabular}{|l|cccc|}
\hline \multirow{4}{*}{ Incentives } & & & $\begin{array}{r}\mathrm{p} \\
\text { (two- } \\
\text { tailed) }\end{array}$ \\
\cline { 2 - 4 } & Df & $\mathrm{F}$ & tain \\
\cline { 2 - 4 } & 2.00 & 0.31 & 0.73 \\
\hline
\end{tabular}

Periods $13-24$

\begin{tabular}{|l|ccc|}
\hline \multirow{4}{*}{ Incentives } & & \multicolumn{2}{c|}{$\begin{array}{c}\mathrm{p} \\
\text { (two- } \\
\text { tailed) }\end{array}$} \\
\cline { 2 - 4 } & Df & $\mathrm{F}$ & tar \\
\cline { 2 - 4 } & 2.00 & 1.36 & 0.26 \\
\hline
\end{tabular}

Periods 25-36

\begin{tabular}{|l|ccc|}
\hline \multirow{4}{*}{ Incentives } & & & $\begin{array}{r}\mathrm{p} \\
\text { (two- }\end{array}$ \\
& Df & F & tailed) \\
\cline { 2 - 4 } & 2.00 & 2.83 & $\mathbf{0 . 0 6}$ \\
\hline
\end{tabular}


Table 20 (continued)

Panel E: Planned Contrasts

\begin{tabular}{|c|rcc|}
\hline & \multicolumn{3}{|c|}{ Average } \\
\hline & \multicolumn{3}{|c}{} \\
& \multicolumn{3}{|c}{} \\
Net Income & \multicolumn{3}{|c|}{$\begin{array}{c}\text { M-value } \\
\text { (one }\end{array}$} \\
Difference & Standard & $\begin{array}{c}\text { Error } \\
\text { tadjusted }^{10}\end{array}$ \\
\hline Periods 4 - 36 & & & \\
Low - Medium & $-53,110.43$ & $26,107.46$ & 0.15 \\
Medium - High & $37,821.14$ & $26,107.46$ & 0.26 \\
Low - High & $-5,289.29$ & $26,500.07$ & 0.50 \\
Periods 4 - 12 & & & \\
Low - Medium & $-6,878.74$ & $9,404.79$ & 0.50 \\
Medium - High & 847.56 & $9,404.79$ & 0.50 \\
Low - High & $-6,031.17$ & $9,546.22$ & 0.50 \\
Periods 13 - 24 & & & \\
Low - Medium & $-14,840.44$ & $11,483.87$ & 0.30 \\
Medium - High & $17,511.93$ & $11,483.87$ & 0.20 \\
Low - High & $2,671.49$ & $11,656.58$ & 0.50 \\
Periods 25 - 36 & & & \\
Low - Medium & $-21,391.25$ & $9,993.73$ & $\mathbf{0 . 0 5}$ \\
Medium - High & $19,461.66$ & $9,993.73$ & $\mathbf{0 . 0 7}$ \\
Low - High & $1,929.59$ & $10,144.02$ & 0.50 \\
\hline
\end{tabular}


Table 20 (continued)

Panel F: Kruskal Wallis Test, Comparing the Effect of All Incentive Conditions on Strategy Accuracy

\begin{tabular}{|c|c|c|c|c|c|c|c|c|}
\hline & \multicolumn{2}{|c|}{ Period $4-36$} & \multicolumn{2}{|c|}{ Period $4-12$} & \multicolumn{2}{|c|}{ Period $13-24$} & \multicolumn{2}{|c|}{ Period $25-36$} \\
\hline & $\begin{array}{c}\text { Chi- } \\
\text { Square } \\
\end{array}$ & $\begin{array}{c}\text { p-value } \\
\text { (two- } \\
\text { tailed) }\end{array}$ & $\begin{array}{l}\text { Chi- } \\
\text { Square }\end{array}$ & $\begin{array}{c}\text { p-value } \\
\text { (two- } \\
\text { tailed) }\end{array}$ & $\begin{array}{c}\text { Chi- } \\
\text { Square }\end{array}$ & $\begin{array}{c}\text { p-value } \\
\text { (two- } \\
\text { tailed) }\end{array}$ & $\begin{array}{c}\text { Chi- } \\
\text { Square }\end{array}$ & $\begin{array}{c}\text { p-value } \\
\text { (two- } \\
\text { tailed) }\end{array}$ \\
\hline Incentives & 2.20 & 0.33 & 1.15 & 0.56 & 2.22 & 0.33 & 2.13 & 0.35 \\
\hline
\end{tabular}

Panel G: Mann Whitney Test, Comparing the Effect of Incentive Conditions on Strategy Accuracy

\begin{tabular}{|c|c|c|c|c|c|c|c|c|}
\hline \multirow[b]{2}{*}{ Incentives } & \multicolumn{2}{|c|}{ Period 4-36 } & \multicolumn{2}{|c|}{ Period $4-12$} & \multicolumn{2}{|c|}{ Period $13-24$} & \multicolumn{2}{|c|}{ Period 25 - 36} \\
\hline & \begin{tabular}{|c} 
Mann- \\
Whitney \\
U
\end{tabular} & $\begin{array}{l}\mathrm{p} \text {-value } \\
\text { (one- } \\
\text { tailed) }\end{array}$ & $\begin{array}{c}\text { Mann- } \\
\text { Whitney } \\
\text { U }\end{array}$ & $\begin{array}{l}\text { p-value } \\
\text { (one- } \\
\text { tailed) }\end{array}$ & $\begin{array}{c}\text { Mann- } \\
\text { Whitney } \\
\text { U }\end{array}$ & $\begin{array}{l}\mathrm{p} \text {-value } \\
\text { (one- } \\
\text { tailed) }\end{array}$ & $\begin{array}{c}\text { Mann- } \\
\text { Whitney } \\
\text { U }\end{array}$ & $\begin{array}{l}\mathrm{p} \text {-value } \\
\text { (one- } \\
\text { tailed) }\end{array}$ \\
\hline & & & & & & 0 & & 0.11 \\
\hline Med & & 0.16 & & & & 0. & & 0.07 \\
\hline Low - High & 480.00 & 0.33 & 448.00 & 0.20 & 504.00 & 0.46 & 498.00 & 0.42 \\
\hline
\end{tabular}

1 In the high complexity conditions, participants were assigned four forward-looking measures to manage, and the effect of the investment on future sales were lagged three periods.

2 When participants earn the maximum bonus available, forward-looking goal attainment is rewarded with $2 / 3$ of the total incentives available while contemporaneous goal attainment is rewarded with $1 / 3$ of the total incentive available. (Figure 1, Conditions A and D)

3 When participants earn the maximum bonus available, forward-looking goal attainment is rewarded with, reward $1 / 3$ of total incentives available while contemporaneous goal attainment is rewarded with $2 / 3$ of the total incentives available. (Figure 1, Conditions B and E)

4 When participants earn the maximum bonus available, contemporaneous goal attainment is rewarded with all of the incentives available. (Figure 1, Conditions $\mathrm{C}$ and $\mathrm{F}$ )

5 In the control condition, participants were paid of flat wage of 600 Lira per period.

6 Due to the lagged effect of investments on net income three periods, the first three periods of operations does not reflect the quality of participants' decisions, and as a result, has been excluded from this analysis.

7 Due to the violation of the sphericity assumption, the p-values are adjusted using the Huynh and Feldt correction factor (Keppel 1973).

8 Thirty-three simulation periods are included in this analysis (i.e., Periods 4-36).

9 Three incentive contracts are included in this analysis (i.e., incentives with low, medium and high weightings on contemporaneous measure.)

10 Planned contrast statistics are corrected using the Bonferroni correction factor. 
Table 21

Mediation Tests - High Complexity Condition ${ }^{1}$

Panel A: Mediation Analysis, Medium and High Contemporaneous Weight Incentive Contracts (Conditions E and F)

Correlations

\begin{tabular}{|c|c|c|c|c|}
\hline & $\begin{array}{c}\text { Mean } \\
\text { Commitmen } \\
\text { Difference } \\
\text { Score, } \\
\text { Period 12 }\end{array}$ & $\begin{array}{c}\text { Mean } \\
\text { Commitment } \\
\text { Difference } \\
\text { Score, } \\
\text { Period } 24\end{array}$ & $\begin{array}{l}\text { Mean Net } \\
\text { Income, } \\
\text { Periods } \\
13-24\end{array}$ & $\begin{array}{c}\text { Mean Net } \\
\text { Income, } \\
\text { Periods } \\
25-36\end{array}$ \\
\hline Mean Commitment Difference Score ${ }^{2}$, Period 12 & 1.00 & & & \\
\hline Mean Commitment Difference Score, Period 24 & $0.38 * *$ & 1.00 & & \\
\hline Cumulative Net Income, Periods 13 - 24 & -0.09 & 0.10 & 1.00 & \\
\hline Cumulative Net Income, Periods 25 - 36 & -0.15 & 0.13 & $0.81 * *$ & 1.00 \\
\hline
\end{tabular}

** Correlation is significant at $\mathrm{p}<0.01$

* Correlation is significant at $\mathrm{p}<0.05$

Period 24 Mediation Analysis Standardized Coefficients (p-value, one-tailed) ( $n=67)$

\begin{tabular}{|l|ccc|}
\hline & \multicolumn{3}{|c|}{ Dependent Variables } \\
\hline & $(1)^{3}$ & $(2)$ & $(3)$ \\
& Cumulative & Commitment & Cumulative \\
Net Income & Difference & Net Income \\
Periods & Score & Periods \\
Incentives & $25-36$ & Period 24 & $25-36$ \\
\cline { 2 - 4 } Commitment Difference Score, Period 24 $^{4}$ & $-0.26(0.02)$ & $-0.04(0.36)$ & $-0.26(0.02)$ \\
Adjusted $\mathrm{R}^{2}$ & & & $0.12(0.16)$ \\
Mediation Condition Satisfied & 0.26 & 0.04 & 0.23 \\
\hline
\end{tabular}


Table 21 (continued)

Panel B: Mediation Analysis, Low and Medium Contemporaneous Weight Incentive Contracts (Conditions D and E)

Correlations

\begin{tabular}{|c|c|c|c|c|}
\hline & \begin{tabular}{|c} 
Mean \\
Commitmen \\
Difference \\
Score, \\
Period 12
\end{tabular} & $\begin{array}{c}\text { Mean } \\
\text { Commitment } \\
\text { Difference } \\
\text { Score, } \\
\text { Period } 24\end{array}$ & $\begin{array}{c}\text { Mean Net } \\
\text { Income, } \\
\text { Periods } \\
13-24\end{array}$ & $\begin{array}{l}\text { Mean Net } \\
\text { Income, } \\
\text { Periods } \\
25-36\end{array}$ \\
\hline Mean Commitment Difference Score ${ }^{2}$, Period 12 & 1.00 & & & \\
\hline Mean Commitment Difference Score, Period 24 & $0.51 * *$ & 1.00 & & \\
\hline Cumulative Net Income ${ }^{3}$, Periods $13-24$ & 0.24 & $0.28 * *$ & 1.00 & \\
\hline Cumulative Net Income, Periods 25 - 36 & 0.17 & $0.32 * *$ & $0.64 * *$ & 1.00 \\
\hline
\end{tabular}

** Correlation is significant at $\mathrm{p}<0.01$

* Correlation is significant at $p<0.05$

Period 24 Mediation Analysis Standardized Coefficients ( $\mathrm{p}$-value, one-tailed) ( $\mathrm{n}=70$ )

\begin{tabular}{|l|ccc|}
\hline & \multicolumn{3}{|c|}{ Dependent Variables } \\
\hline & $(1)^{3}$ & $(2)$ & $(3)$ \\
& Cumulative & Commitment & Cumulative \\
Net Income & Difference & Net Income \\
& Periods & Score & Periods \\
& $25-36$ & Period 24 & $25-36$ \\
\cline { 2 - 4 } & $0.28(0.01)$ & $0.32(<0.01)$ & $0.23(0.06)$ \\
Incentives $^{5}$ & & & $0.27(0.02)$ \\
Commitment Difference Score, Period $24^{\text {Adjusted } \mathrm{R}^{2}}$ & 0.28 & 0.32 & 0.39 \\
Mediation Condition Satisfied & Yes & Yes & Yes \\
\hline
\end{tabular}




\section{Table 21 (continued)}

In the high complexity conditions, participants were assigned four forward-looking measures to manage, and the effect of the investment on future sales were lagged three periods.

2 Commitment difference score is the difference between contemporaneous and forward-looking goal commitment, where a positive number indicates higher levels of contemporaneous goal commitment, and a negative number indicates higher levels of forward-looking goal commitment.

3

To test mediation, I estimate three regressions, as specified by Baron and Kenny (1986). The three regressions are as follows:

Cumulative Net Income $=\mathrm{a} 0+\mathrm{a} 1 *$ Incentive Contract Condition

Commitment Difference Score $=\mathrm{b} 0+\mathrm{b} 1 *$ Incentive Contract Condition

Cumulative Net Income $=\mathrm{c} 0+\mathrm{c} 1 *$ Incentive Contract Condition + c2 $*$ Commitment Difference Score

4 The dummy variable is equal to 1 if the high contemporaneous weighting incentive contract was assigned, and 0 if it was not.

5 The dummy variable is equal to 1 if the medium contemporaneous weighting incentive contract was assigned, and 0 if it was not. 
Table 22

Analysis of Control Conditions

Panel A: Cumulative Net Income, Low Complexity Condition ${ }^{1}$

\begin{tabular}{|c|c|c|c|}
\hline Incentive Contract & $\begin{array}{c}\text { Mean } \\
\text { Difference }\end{array}$ & $\begin{array}{c}\text { Standard } \\
\text { Error }\end{array}$ & $\begin{array}{c}\text { p-value } \\
\text { (two- } \\
\text { tailed, } \\
\text { adjusted }^{2} \text { ) }\end{array}$ \\
\hline \multicolumn{4}{|l|}{ Periods $2-36^{3}$} \\
\hline Low $^{4}-$ Control $^{5}$ & $10,615.50$ & $8,844.35$ & 1.00 \\
\hline Medium $^{6}$ - Control & -647.94 & $8,780.08$ & 1.00 \\
\hline $\mathrm{High}^{7}$ - Control & $-6,101.99$ & $9,059.44$ & 1.00 \\
\hline \multicolumn{4}{|l|}{ Periods 2 - 12} \\
\hline Low - Control & $1,725.69$ & $3,027.95$ & 1.00 \\
\hline Medium - Control & $-1,274.98$ & $3,005.34$ & 0.66 \\
\hline High - Control & -637.53 & $3,101.58$ & 1.00 \\
\hline \multicolumn{4}{|l|}{ Periods 13 - 24} \\
\hline Low - Control & $3,080.91$ & $3,436.96$ & 1.00 \\
\hline Medium - Control & $(46.17)$ & $3,411.99$ & 1.00 \\
\hline High - Control & $-2,878.56$ & $3,520.55$ & 1.00 \\
\hline \multicolumn{4}{|l|}{ Periods $25-36$} \\
\hline Low - Control & $5,808.90$ & $3,401.09$ & 0.39 \\
\hline Medium - Control & 673.21 & $3,376.37$ & 1.00 \\
\hline High - Control & $-2,585.91$ & $3,483.80$ & 1.00 \\
\hline
\end{tabular}

Panel B: Task Complexity, Low Complexity Condition

\begin{tabular}{|c|ccc|}
\hline & & & \\
p-value \\
(two- \\
Incentive Contract & $\begin{array}{c}\text { Mean } \\
\text { Difference }\end{array}$ & $\begin{array}{c}\text { Standard } \\
\text { Error }\end{array}$ & $\begin{array}{c}\text { tailed, } \\
\text { adjusted }\end{array}$ \\
\hline Low - Control & -0.51 & 0.25 & 0.13 \\
Medium - Control & -0.57 & 0.24 & $\mathbf{0 . 0 7}$ \\
High - Control & -0.82 & 0.25 & $\mathbf{0 . 0 1}$ \\
\hline
\end{tabular}


Table 22 (continued)

Panel C: Cumulative Net Income, High Complexity Condition ${ }^{8}$

\begin{tabular}{|c|ccc|}
\hline & & & \\
& Mean & Standard & $\begin{array}{c}\text { p-value } \\
\text { (two- } \\
\text { tailed, } \\
\text { adjusted) }\end{array}$ \\
\hline Incentive Contract & Difference & Error & \\
Periods 4 - 36 & & & \\
Low - Control & $37,815.37$ & $26,580.22$ & 0.51 \\
Medium - Control & $-5,295.05$ & $26,180.31$ & 1.00 \\
High - Control & $32,526.09$ & $26,580.22$ & 0.84 \\
Periods 4 - 12 & & & \\
Low - Control & $3,754.55$ & $9,782.27$ & 1.00 \\
Medium - Control & $-3,124.19$ & $9,635.09$ & 1.00 \\
High - Control & -2276.64 & $9,782.27$ & 1.00 \\
Periods 13 - 24 & & & \\
Low - Control & $10,572.61$ & $11,190.65$ & 1.00 \\
Medium - Control & $-4,267.83$ & $11,022.28$ & 1.00 \\
High - Control & $13,244.09$ & $11,190.65$ & 0.81 \\
Periods 25 - 36 & & & \\
Low - Control & $23,488.21$ & $10,301.57$ & 0.15 \\
Medium - Control & $2,096.97$ & $10,146.58$ & 1.00 \\
High - Control & $21,558.63$ & $10,301.57$ & 0.18 \\
\hline
\end{tabular}

Panel D: Task Complexity, High Complexity Condition

\begin{tabular}{|c|c|c|c|}
\hline Incentive Contract & $\begin{array}{c}\text { Mean } \\
\text { Difference }\end{array}$ & $\begin{array}{l}\text { Standard } \\
\text { Error }\end{array}$ & $\begin{array}{l}\text { p-value } \\
\text { (one- } \\
\text { tailed, } \\
\text { adjusted }^{2} \text { ) }\end{array}$ \\
\hline Low - Control & -0.08 & 0.24 & 0.50 \\
\hline Medium - Control & 0.13 & 0.24 & 0.50 \\
\hline High - Control & 0.55 & 0.24 & 0.07 \\
\hline
\end{tabular}




\section{Table 22 (continued)}

1 In the low complexity conditions, participants were assigned two forward-looking measures to manage, and the effect of the investment on future sales were lagged one period. Planned contrast statistics are corrected using the Bonferroni correction factor.

Due to the lagged effect of investments on net income one period, the first periods of operations does not reflect the quality of participants' decisions, and as a result, has been excluded from this analysis. When participants earn the maximum bonus available, forward-looking goal attainment is rewarded with $2 / 3$ of the total incentives available while contemporaneous goal attainment is rewarded with $1 / 3$ of the total incentive available. (Figure 1, Conditions A and D)

5 In the control condition, participants were paid a flat wage of 600 Lira.

6 When participants earn the maximum bonus available, forward-looking goal attainment is rewarded with, reward $1 / 3$ of total incentives available while contemporaneous goal attainment is rewarded with $2 / 3$ of the total incentives available. (Figure 1, Conditions B and E)

7 When participants earn the maximum bonus available, contemporaneous goal attainment is rewarded with all of the incentives available. (Figure 1, Conditions $\mathrm{C}$ and F)

8 In the high complexity conditions, participants were assigned four forward-looking measures to manage, and the effect of the investment on future sales were lagged three periods.

9 Due to the lagged effect of investments on net income three periods, the first three periods of operations does not reflect the quality of participants' decisions, and as a result, has been excluded from this analysis. 
Table 23

Summary of Hypotheses Testing

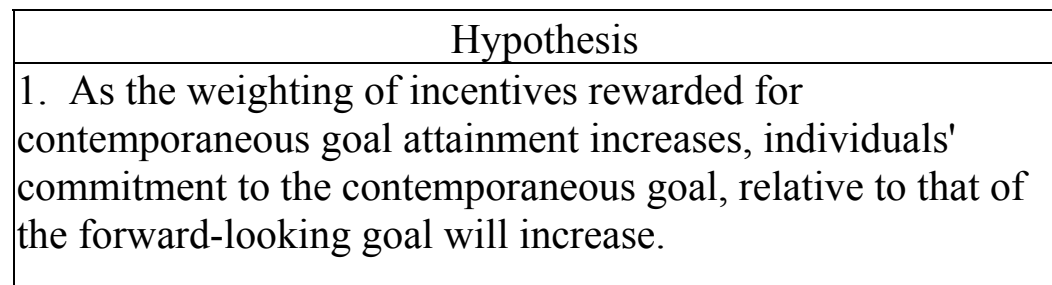

2. In low complexity tasks, as the weighting of incentives rewarded for contemporaneous goal attainment increases individuals will make better investment decisions.

3. In low complexity tasks, as the weighting of incentives rewarded for contemporaneous goal attainment increase, individuals commitment to the contemporaneous goal, relative to that of the forward-looking goal, will mediate the relationship between incentives and performance.

4. In high complexity tasks, individuals who are rewarded for the attainment of both forward-looking and contemporaneous goals will make better investment decisions than those who are rewarded for the attainment of only contemporaneous goals.

5. In high complexity tasks, when individuals are rewarded only for contemporaneous goal attainment or for forwardlooking and contemporaneous goal attainment, individuals' commitment to the contemporaneous goal, relative to that of the forward-looking goal, will mediate the relationship between incentives and performance.

6. In high complexity tasks, individuals will make better investment decisions when incentives rewarded for contemporaneous goal attainment are weighting more heavily than those for forward-looking goal incentives

7. In low complexity tasks, when incentives are rewarded for both contemporaneous and forward-looking goal attainment are weighted more heavily than those for forward-looking goal incentives, individuals commitment to the contemporaneous goal, relative to that of the forward-looking goal, will mediate the relationship between incentives and performance.

Supported

Not Supported

Partially

Supported

Not Supported

Supported

Not Supported

Supported

Supported 


\section{APPENDIX}

\section{Experimental Instrument}

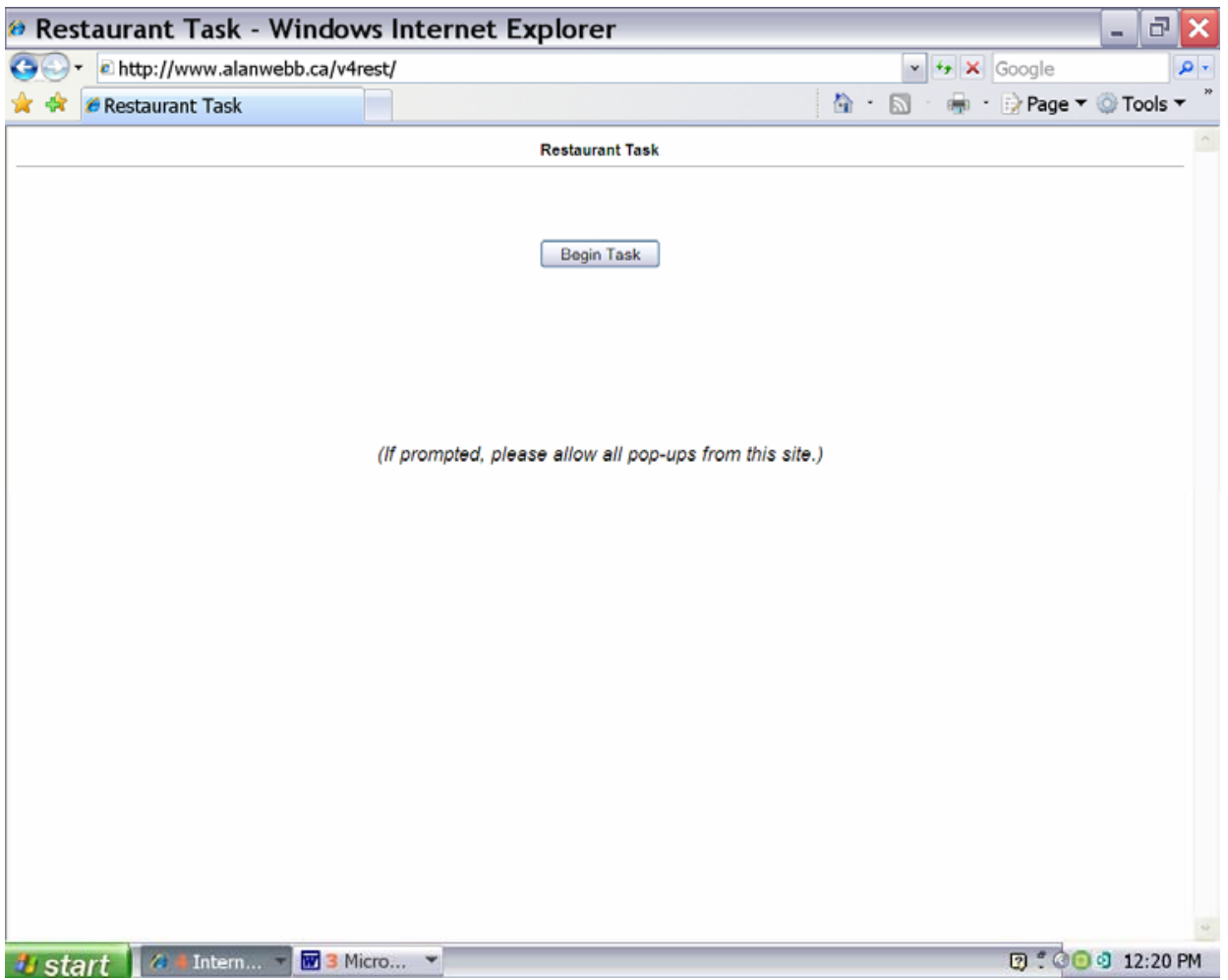

a http://www.alanwebb.ca/ - Restaurant Task - Windows Internet Explorer - -

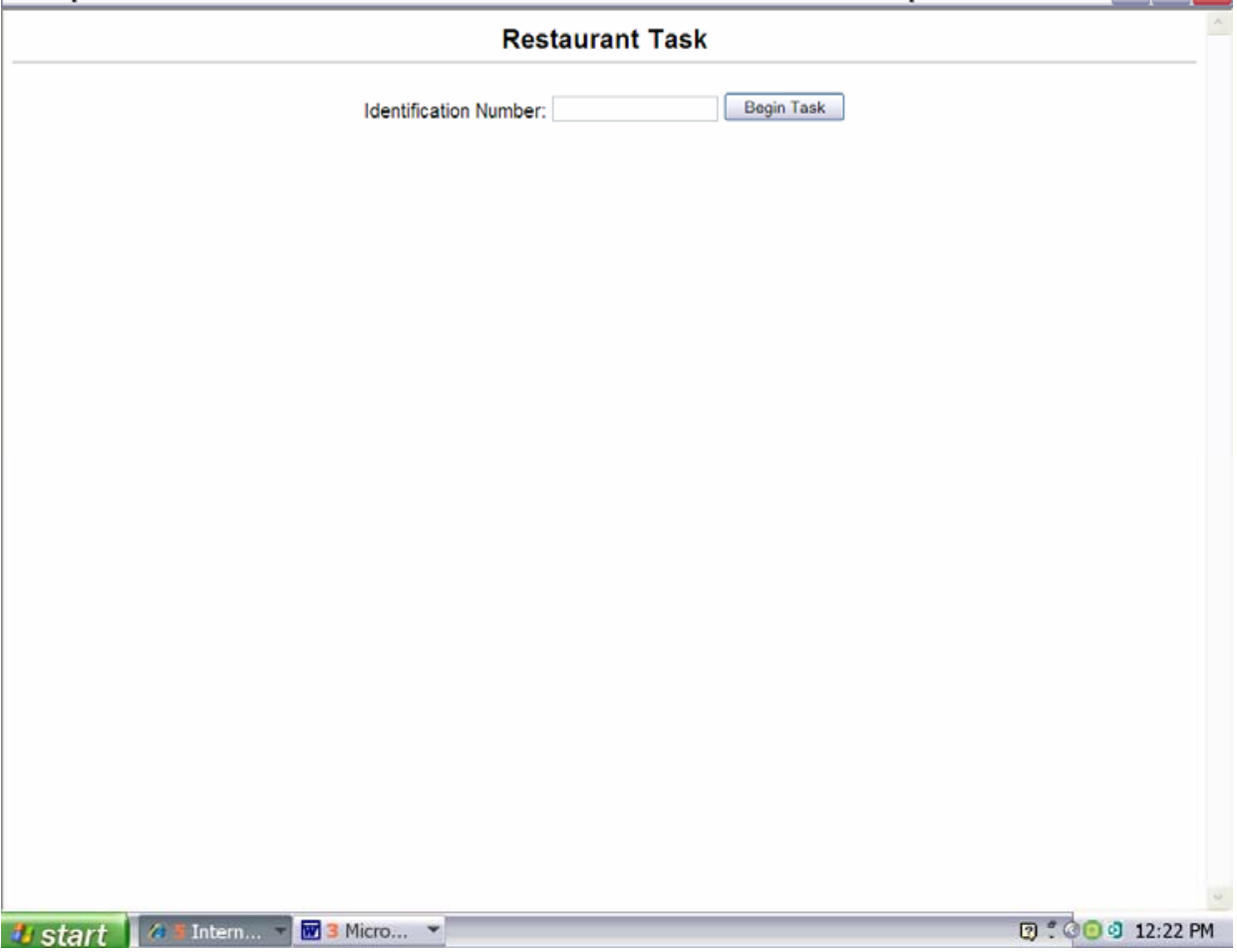



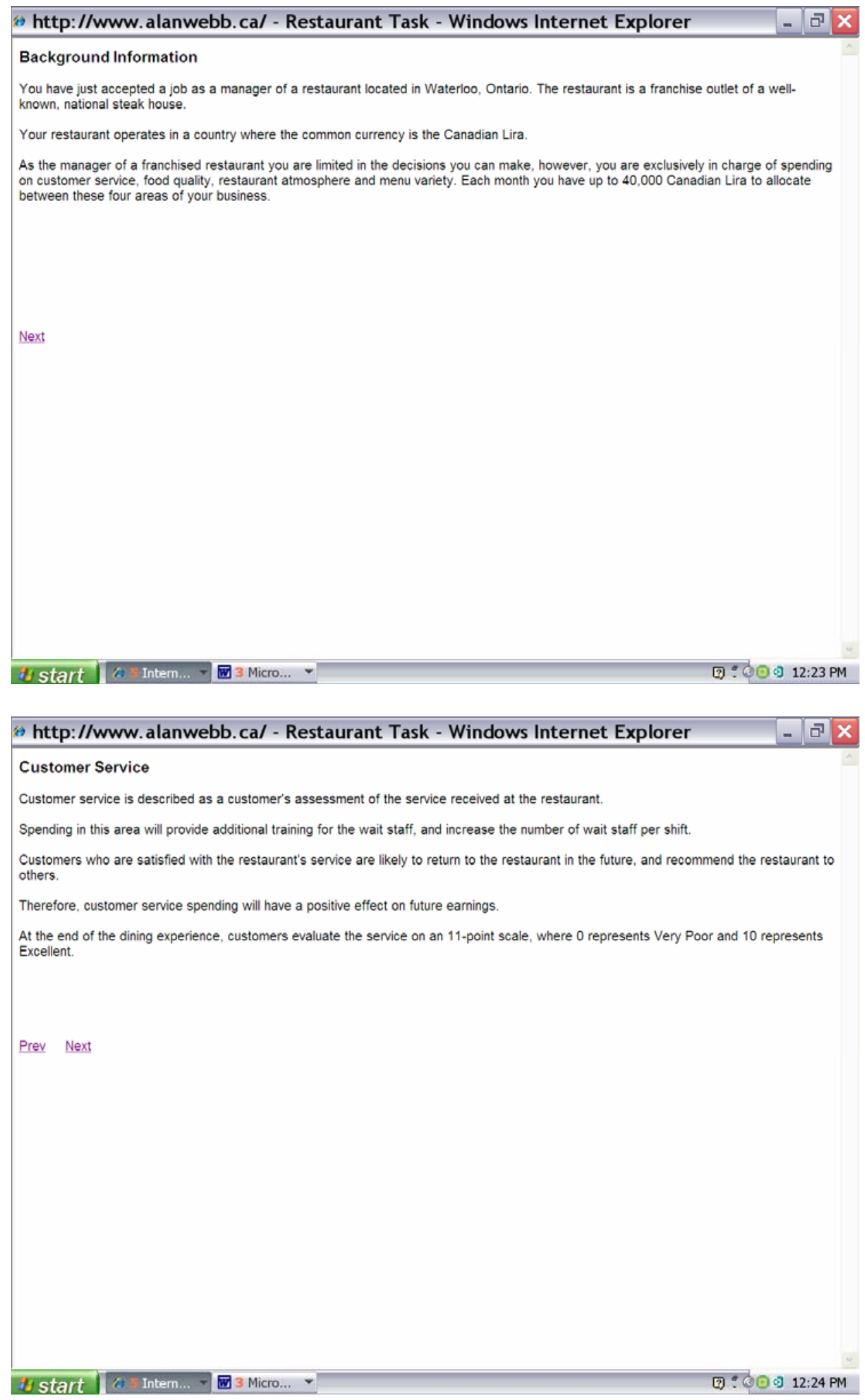


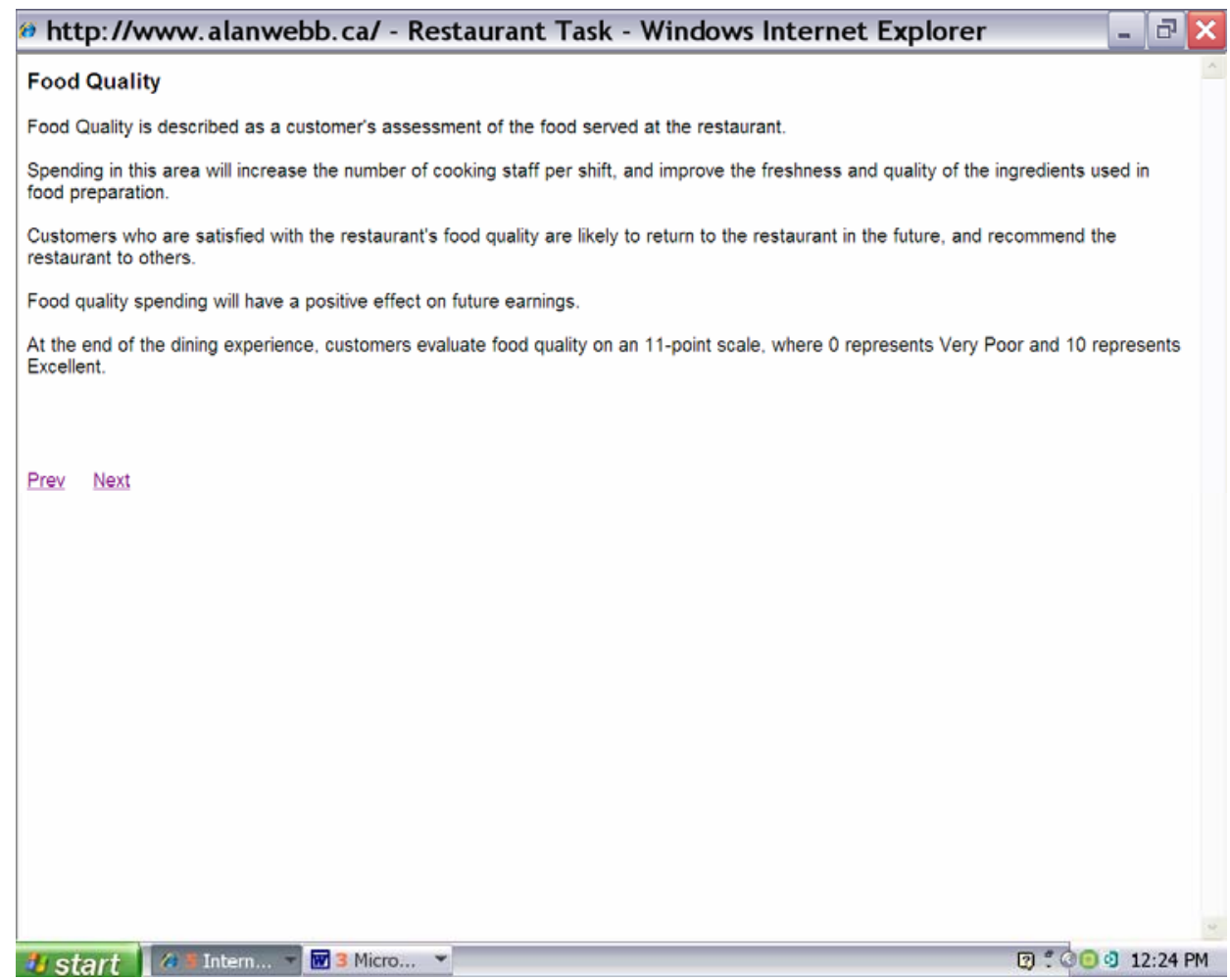

\section{a http://www.alanwebb.ca/ - Restaurant Task - Windows Internet Explorer $-\square \times$}

Restaurant Atmosphere

Restaurant Atmosphere is the customer's satisfaction with the appearance of the restaurant.

Spending in this area will provide additional maintenance of the restaurant, including finer table linens and more attractive wait staff uniforms.

Customers who are satisfied with the restaurant's atmosphere are likely to return to the restaurant in the future, and recommend the restaurant to others.

Therefore, spending on the restaurant's atmosphere will have a positive effect on future earnings

At the end of the dining experience, customers evaluate the service on an 11-point scale, where 0 represents Very Poor and 10 represents Excellent.

Prev Next 


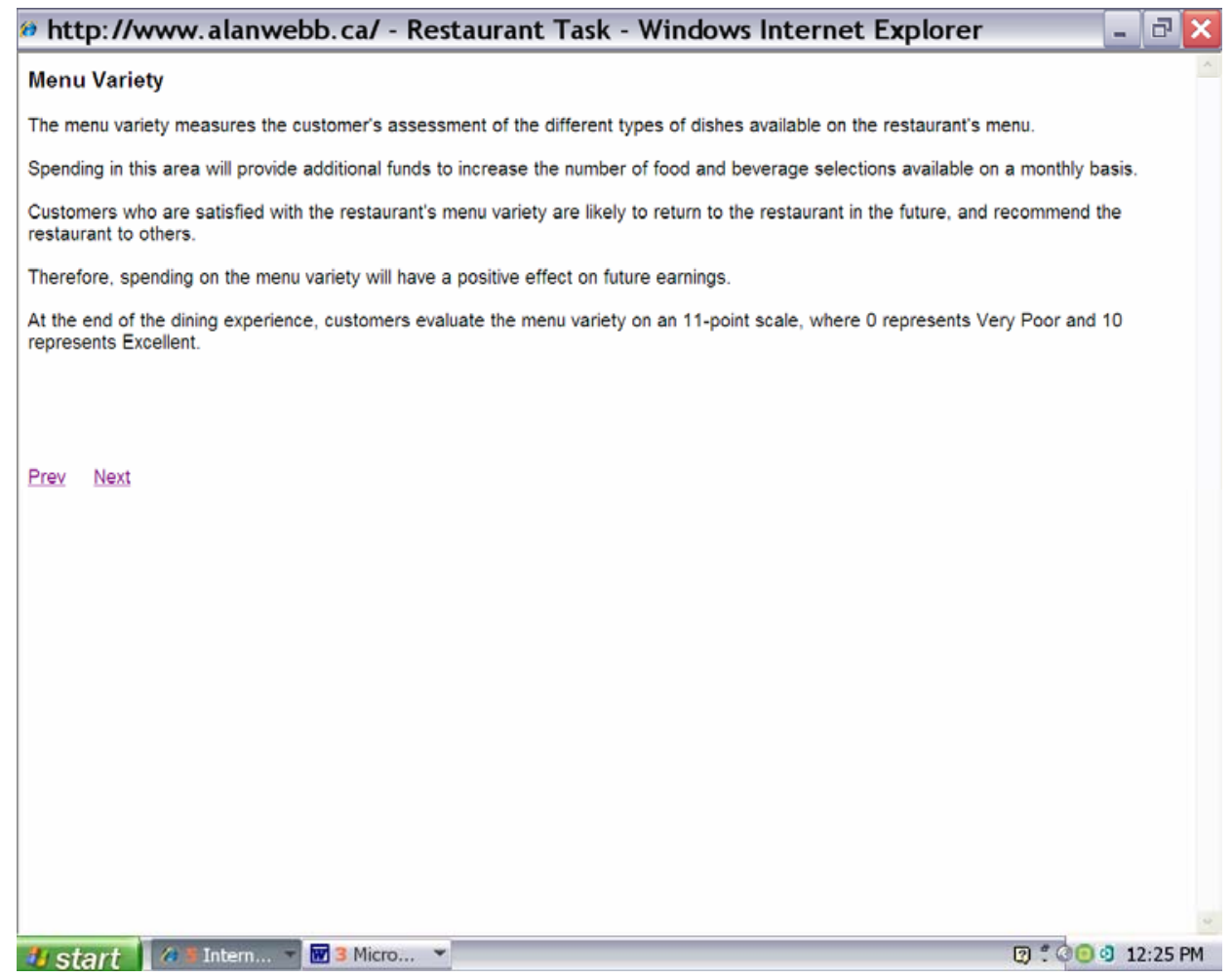

a http://www. alanwebb.ca/ - Restaurant Task - Windows Internet Explorer - -

Food Quality, Customer Service, Restaurant Atmosphere and Menu Variety

How do you think food quality, customer service, restaurant atmosphere and menu variety contribute to a restaurant's success?

Please allocate 100 percentage points between food quality, customer service, restaurant atmosphere and menu variety to demonstrate your opinion about their relative importance. (For example, allocating 25 points to each investment means that you believe that they are all equally important to a restaurant's success.)

\begin{tabular}{|c|c|}
\hline Customer Service: & $\%$ \\
\hline Food Quality: & $\%$ \\
\hline Restaurant Atmosphere: & $\%$ \\
\hline Menu Variety: & $\%$ \\
\hline
\end{tabular}

Submit 

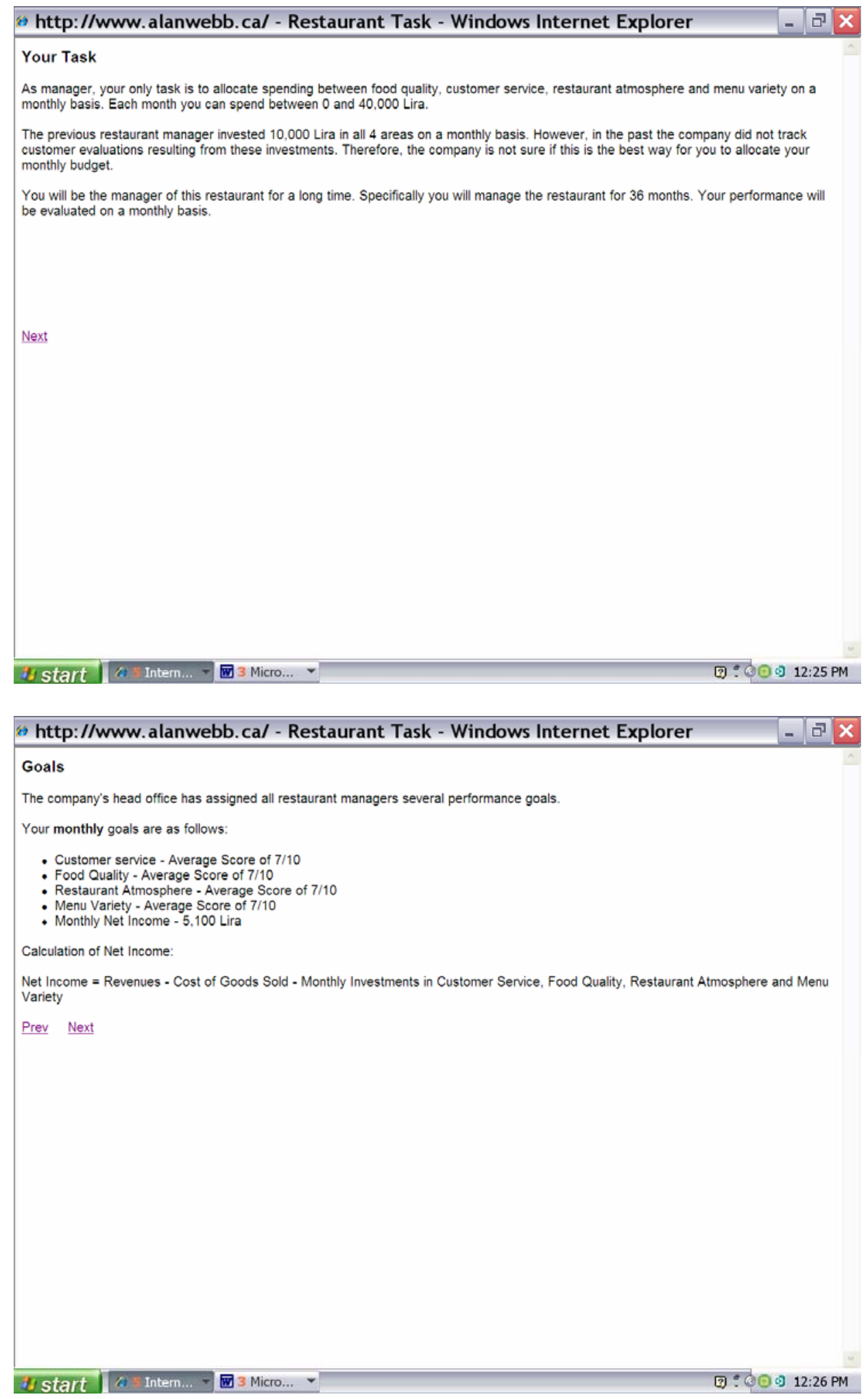


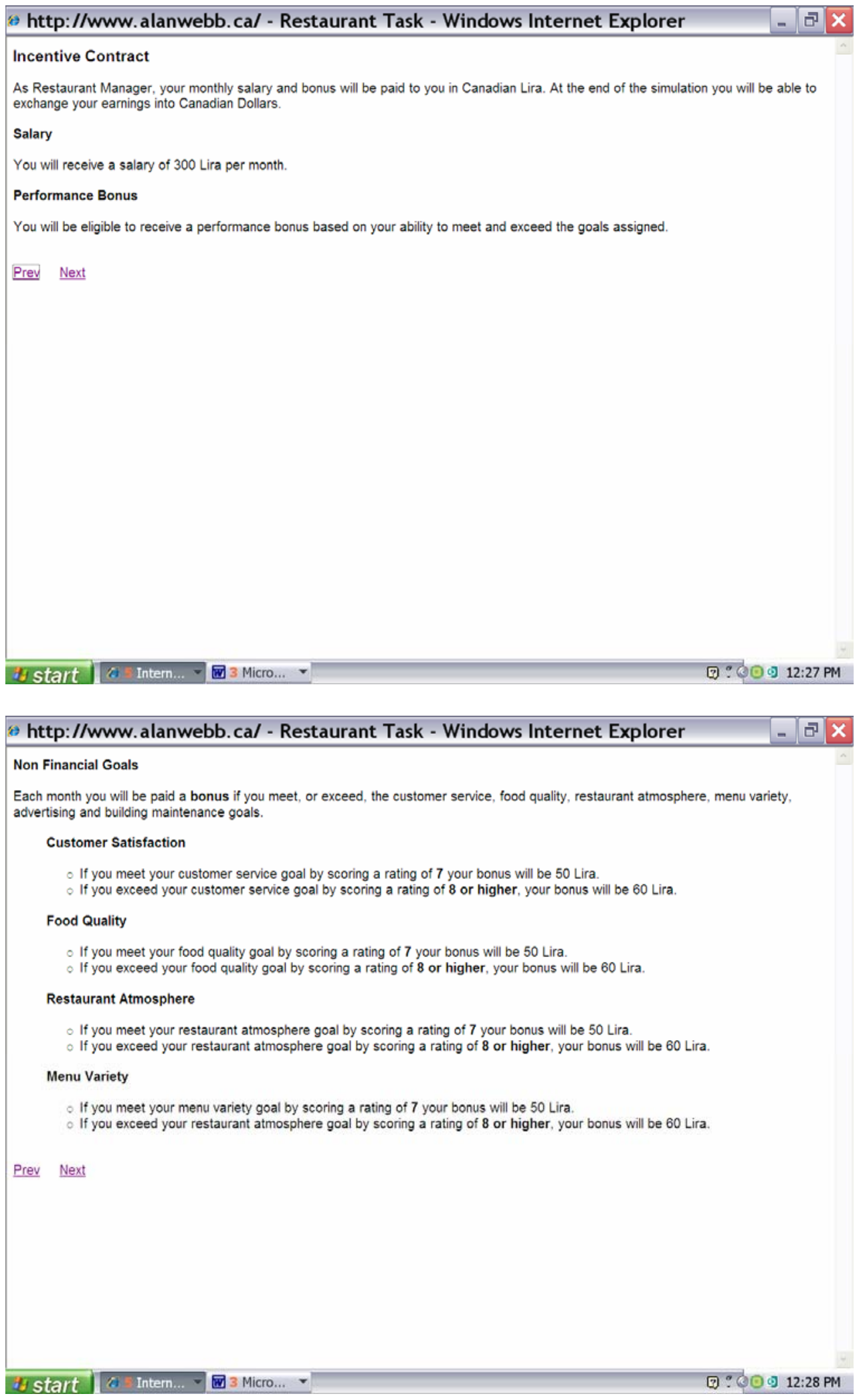




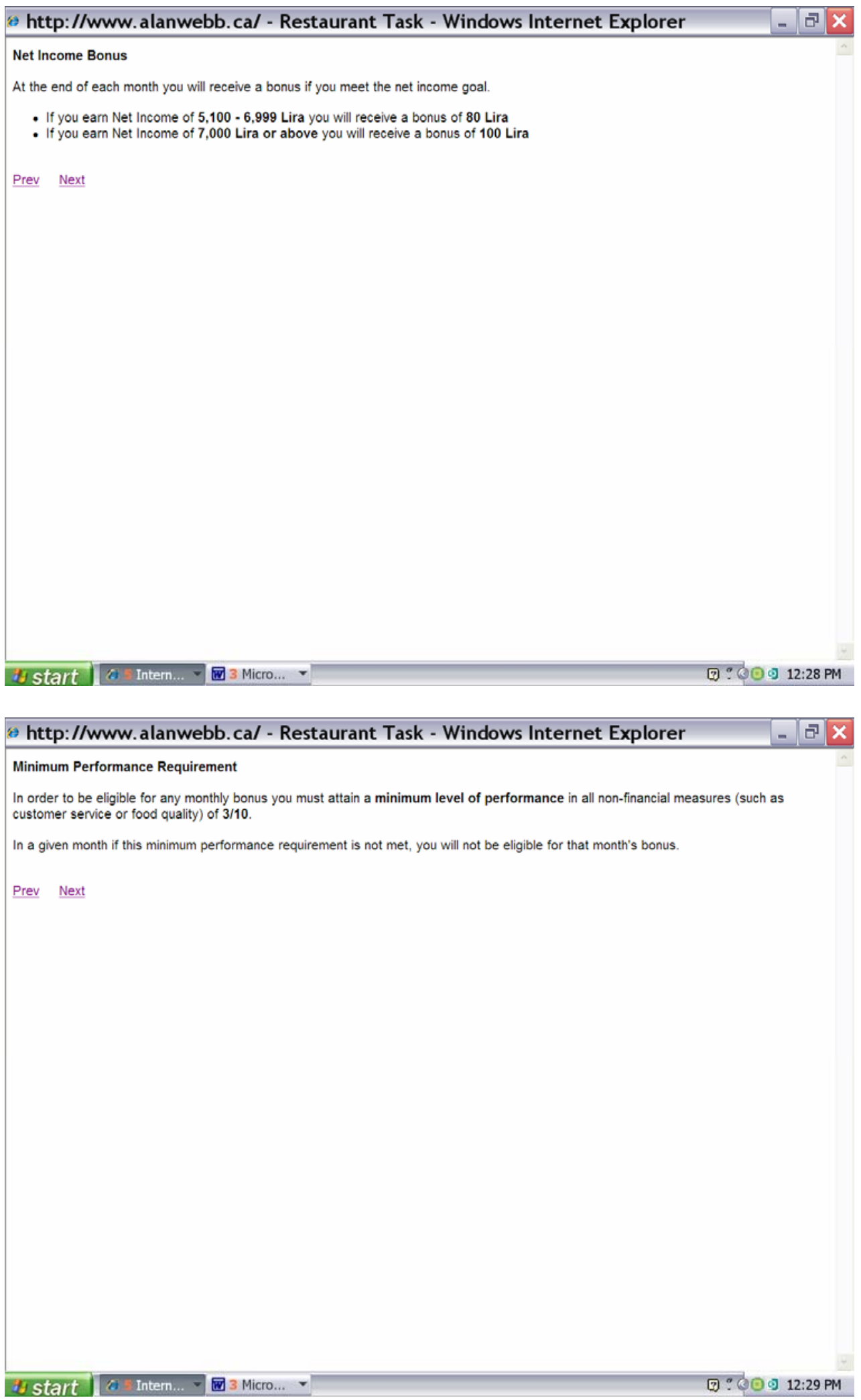




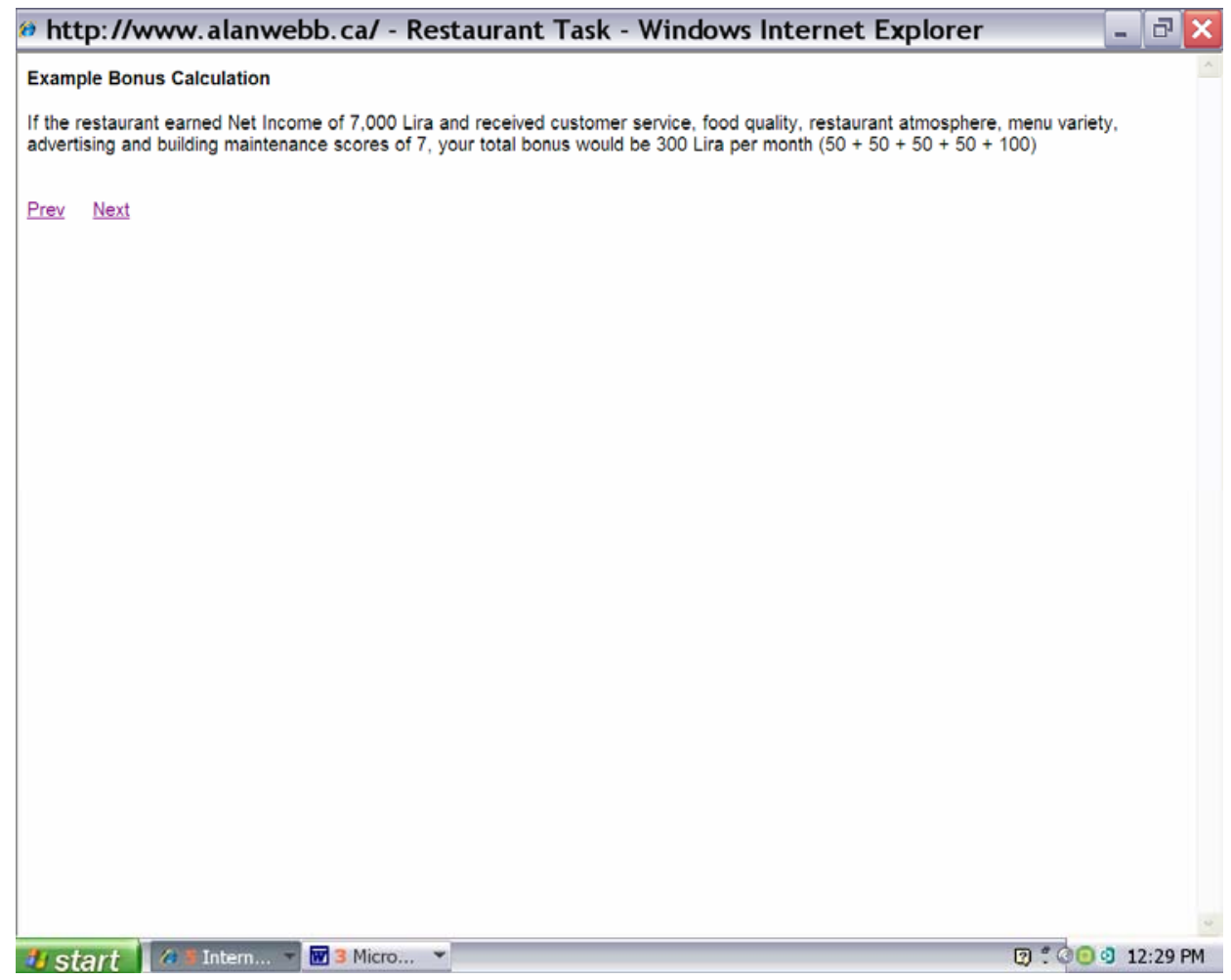

o http://www. alanwebb.ca/ - Restaurant Task - Windows Internet Explorer

-

Information Available by Clicking on Data Links

During the simulation the following links will appear on your screen. At any time you may click on any of them to review the following information:

1. Prior Investments and Performance

Prior periods investment decisions; customer service, food quality, restaurant atmosphere, and menu variety scores; and total net income.

2. Incentive Contract Details

Information about your flat wage

3. Goals

Information on the goals assigned

4. Task

Information on the investment task

5. Background Information

General information on the company, and the non-financial measures.

Prev Next 

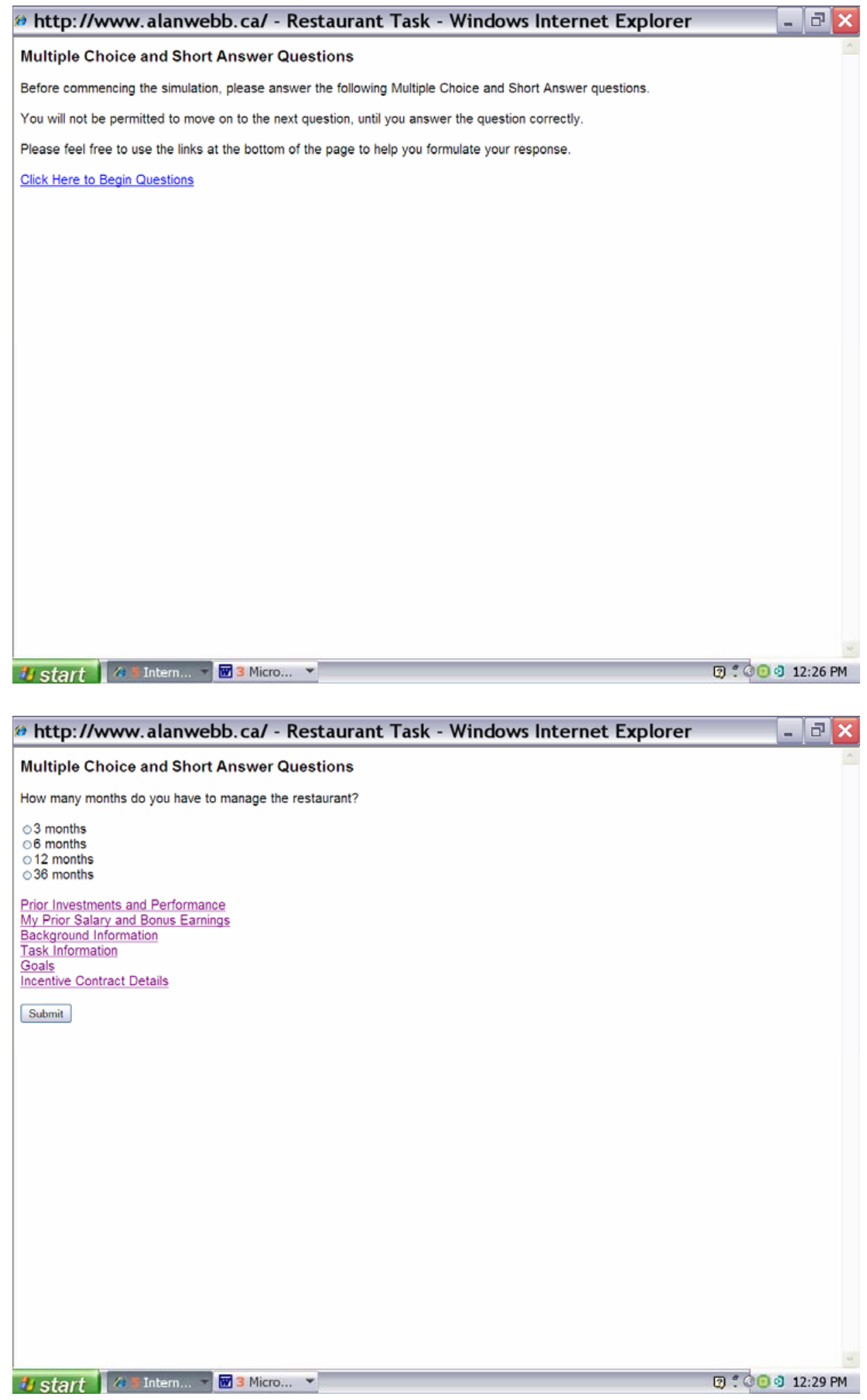


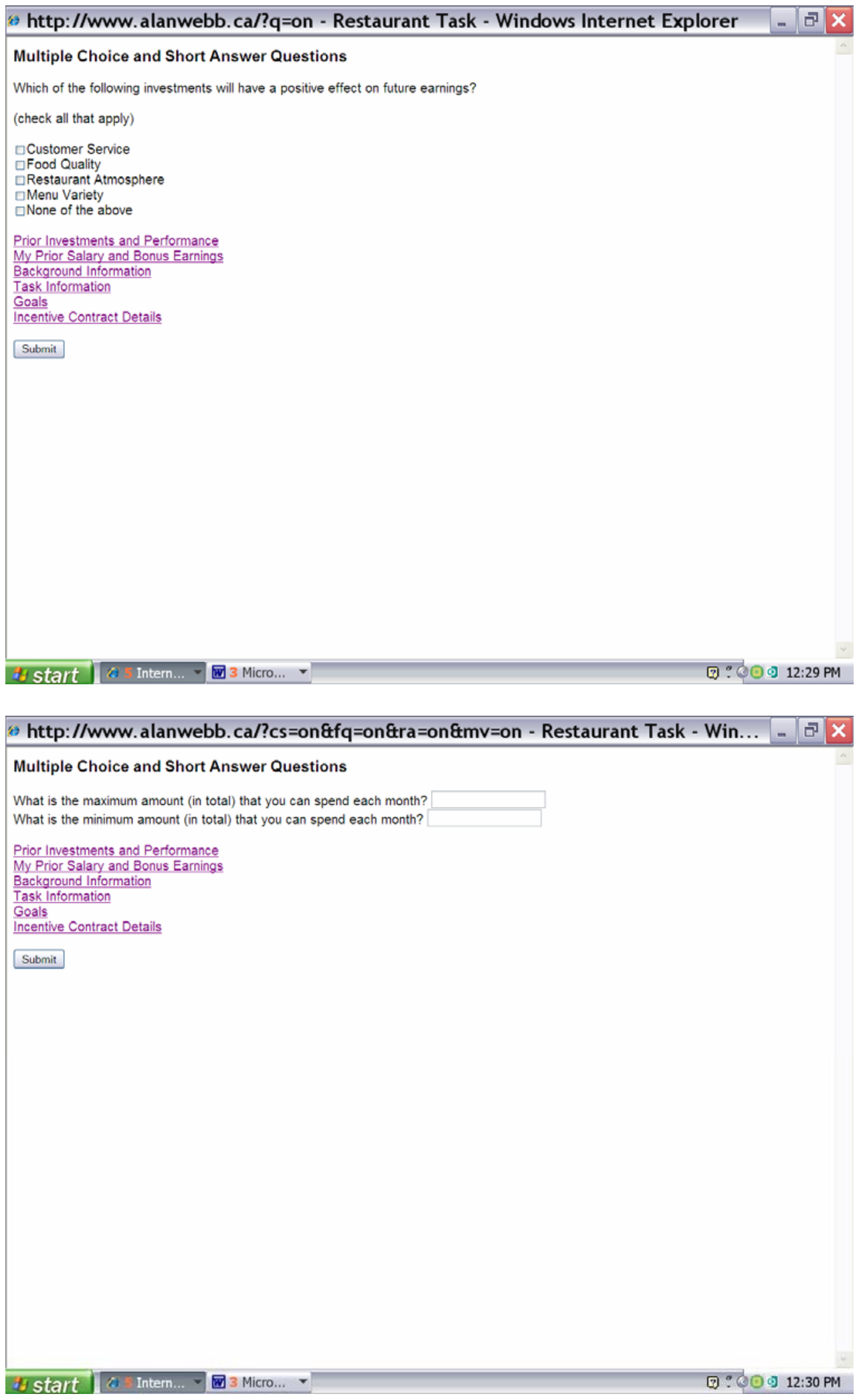




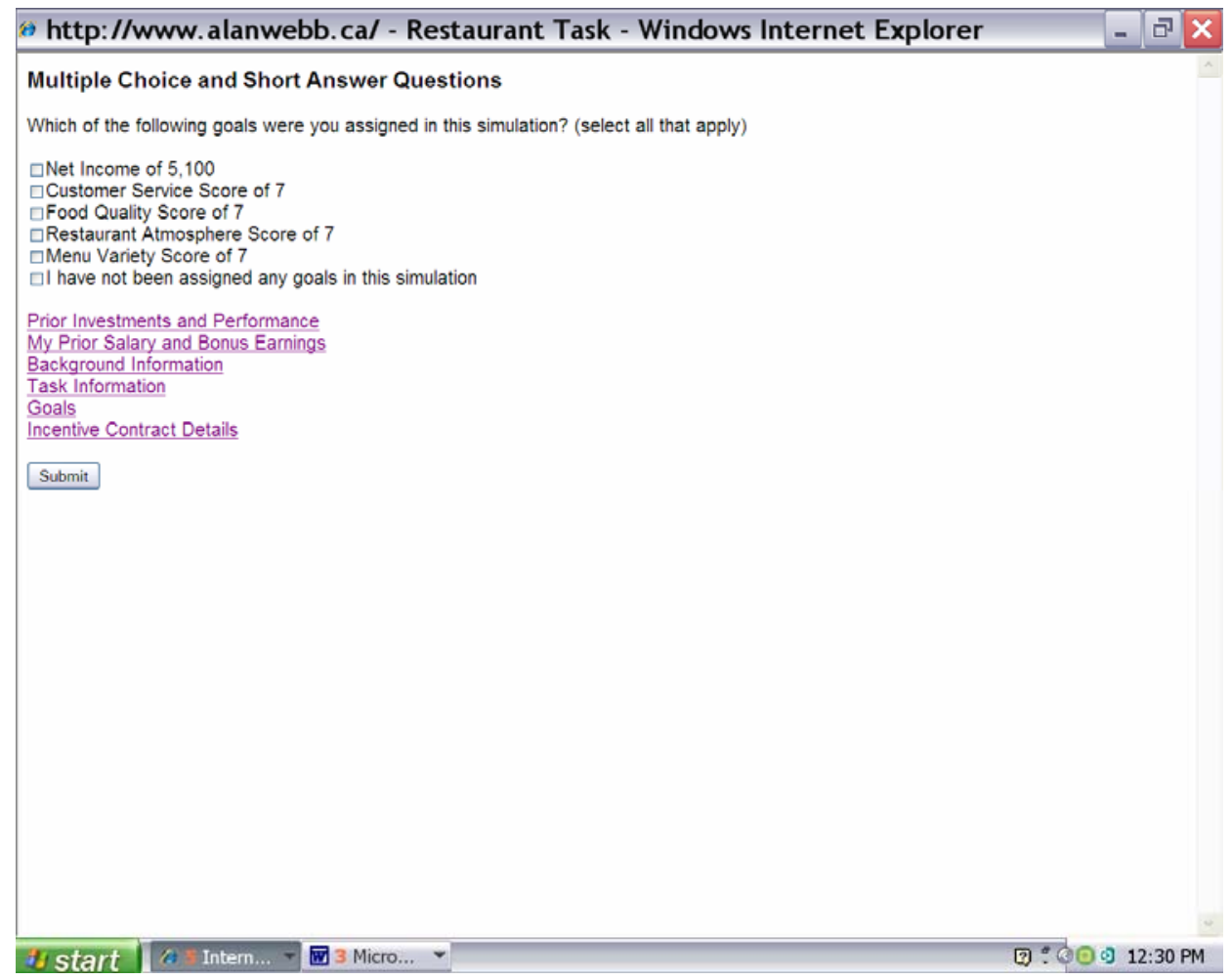

a http://www. alanwebb. $c a / ? a=o n \& b=o n \& c=o n \& d=o n \& t=o n-$ Restaurant Task $-W \ldots-a$ Multiple Choice and Short Answer Questions

Given the following levels of performance, calculate the bonus you would earn based on your monthly Net Income result.

(Hint: Consider only your bonus payment, not your monthly salary, when answering this question.)

Net Income of 3,000 Lira:

Net Income of 5,100 Lira

Net Income of 7,000 Lira:

Prior Investments and Performance

My Prior Salary and Bonus Earnings

Background Information

Task Information

Incentive Contract Details

Submit 


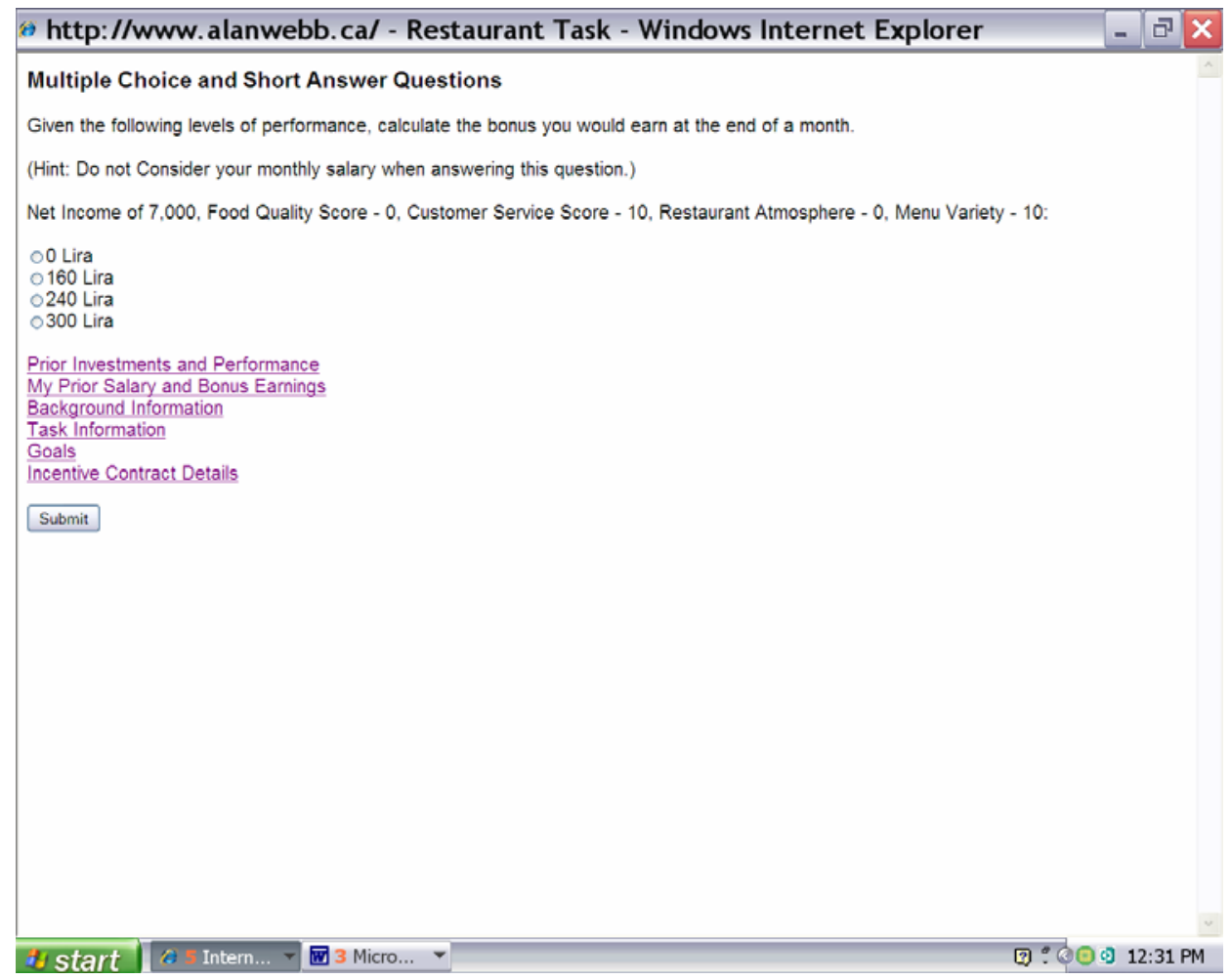

a http://www. alanwebb.ca/?q=on - Restaurant Task - Windows Internet Explorer - $\square$ Multiple Choice and Short Answer Questions

If the restaurant received a customer service score of 7 , a food quality score of 7 , a restaurant atmosphere score of 7 , a menu variety score of 7 and a Net Income of 5,500 Lira, calculate the bonus you would earn at the end of a month

Prior Investments and Performance

My Prior Salary and Bonus Earning

Background Information

Task Information

Goals

Incentive Contract Details

Submit 


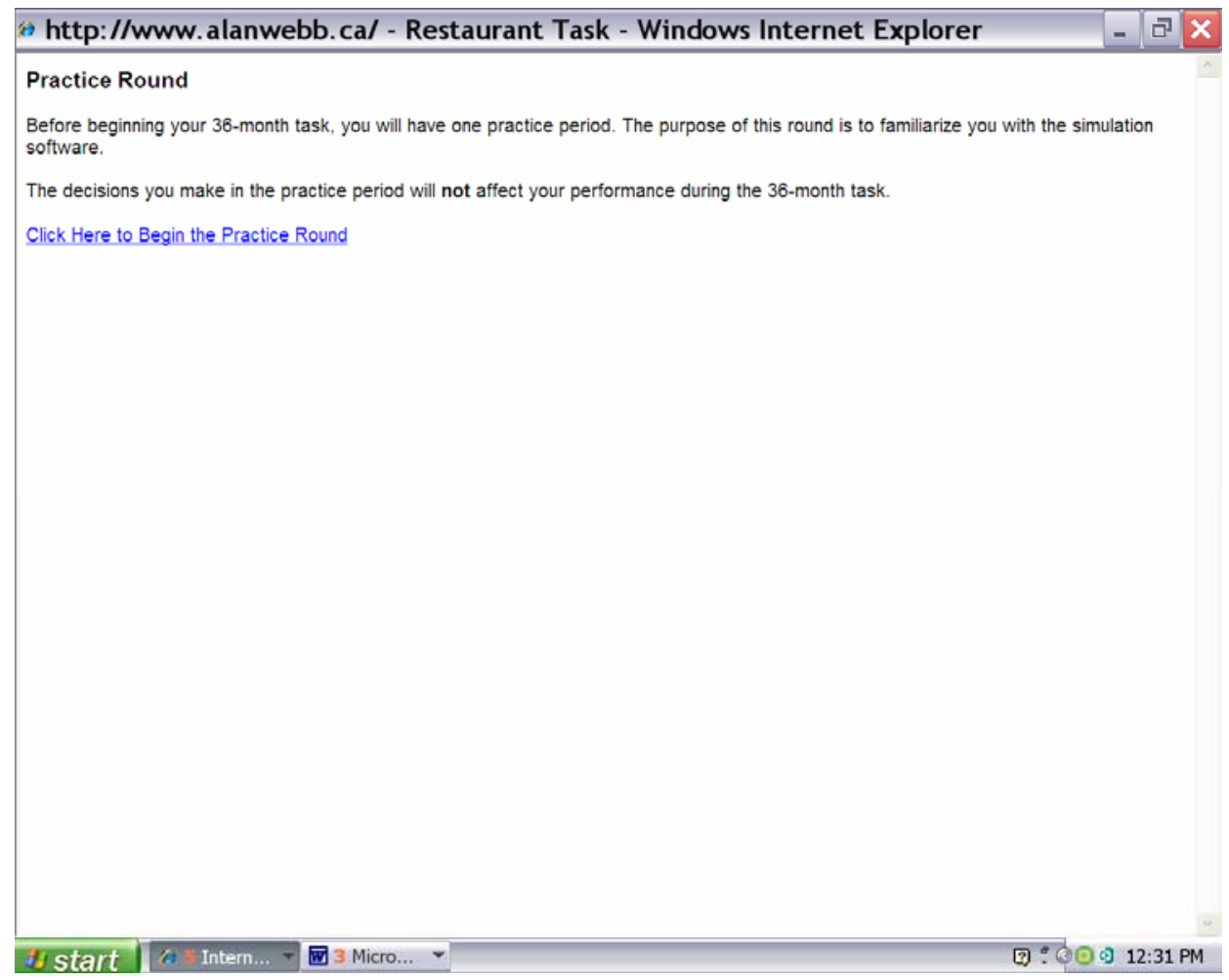

a http://www.alanwebb.ca/ - Restaurant Task - Windows Internet Explorer - 므

Practice Round

You have a budget of 40,000 Lira this month. How much would you like to invest in customer service, food quality, restaurant atmosphere, and menu variety?

Customer Service:

Food Quality:

Restaurant Atmosphere:

Menu Variety:

Prior Investments and Performance

My Prior Salary and Bonus Earnings

Background Information

Task Information

Incentive Contract Details

Submit 


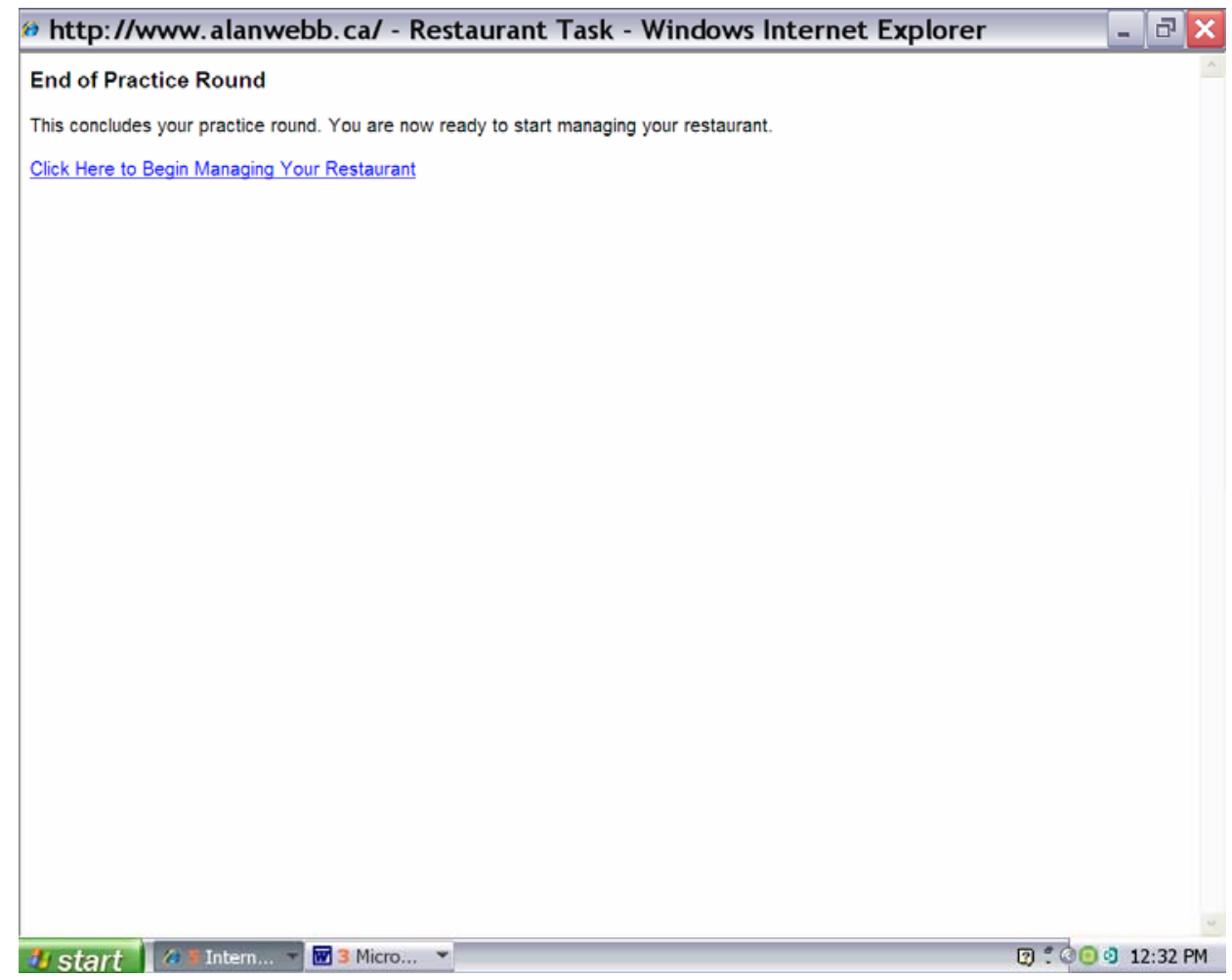

a http://www.alanwebb.ca/ - Restaurant Task - Windows Internet Explorer - 마

Month 1 of 36

You have a budget of 40,000 Lira this month. How much would you like to invest in customer service, food quality, restaurant atmosphere, and menu variety?

Customer Service:

Food Quality:

Restaurant Atmosphere:

Menu Variety:

Prior Investments and Performance

My Prior Salary and Bonus Earnings

Background information

Task Information

Incentive Contract Details

Submit 


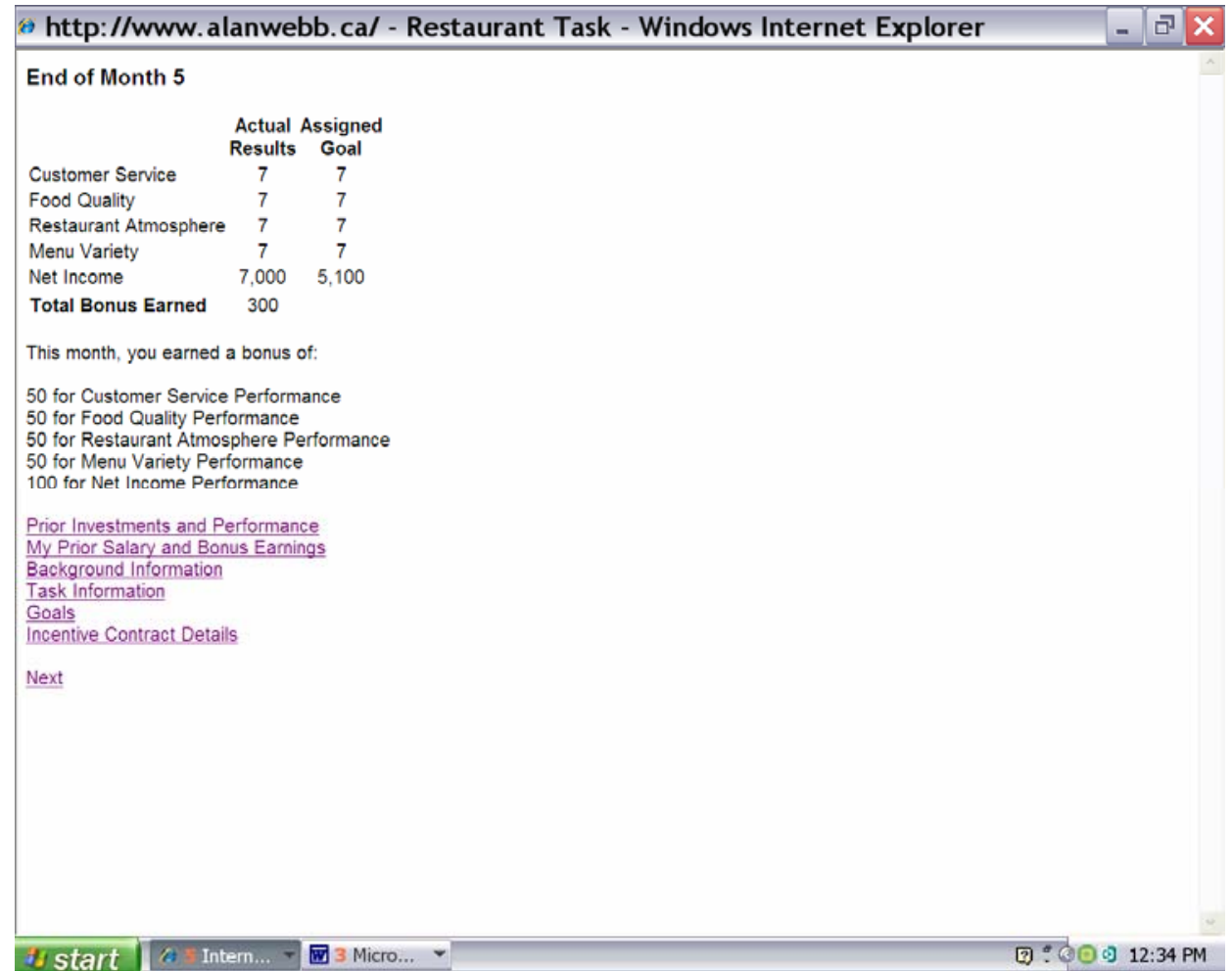

\begin{tabular}{|c|c|c|c|c|c|c|c|c|}
\hline \multicolumn{7}{|c|}{ \& http://www.alanwebb.ca/ - Restaurant Task - Windows Internet Explorer } & - & $\mathbf{x}$ \\
\hline \multicolumn{8}{|c|}{$\begin{array}{l}\text { Before continuing with the simulation, please answer the following questions. Once you have completed all } \\
\text { questions, you will be able to resume the simulation. }\end{array}$} & $\hat{\imath}$ \\
\hline \multicolumn{9}{|c|}{$\begin{array}{l}\text { The following questions are about your non-financial goals of } 7 \text { (Customer Service, Food Quality, Restaurant Atmosphere and } \\
\text { Menu Variety) }\end{array}$} \\
\hline \multicolumn{9}{|c|}{ 1. It's hard to take the non-financial goals (scores of 7 ) seriously. } \\
\hline$\circ$ & $\circ$ & $\circ$ & $\circ$ & $\circ$ & $\circ$ & $\circ$ & & \\
\hline-3 & -2 & -1 & 0 & 1 & 2 & 3 & & \\
\hline $\begin{array}{l}\text { Strongly } \\
\text { Disagree }\end{array}$ & Disagree & $\begin{array}{l}\text { Somewhat } \\
\text { Disagree }\end{array}$ & $\begin{array}{l}\text { Neither Agree } \\
\text { Nor Disagree }\end{array}$ & $\begin{array}{l}\text { Somewhat } \\
\text { Agree }\end{array}$ & Agree & $\begin{array}{l}\text { Strongly } \\
\text { Agree }\end{array}$ & & \\
\hline \multicolumn{9}{|c|}{ 2. Quite frankly, I don't care if I achieve the non-financial goals (scores of 7) or not. } \\
\hline 0 & 0 & 0 & 0 & 0 & 0 & 0 & & \\
\hline-3 & -2 & -1 & 0 & 1 & 2 & 3 & & \\
\hline Strongly & Disagree & Somewhat & Neither Agree & Somewhat & Agree & Strongly & & \\
\hline Disagree & & Disagree & Nor Disagree & Agree & & Agree & & \\
\hline \multicolumn{9}{|c|}{ 3. I am strongly committed to pursuing the non-financial goals (scores of 7 ). } \\
\hline 0 & 0 & $\circ$ & 0 & 0 & 0 & 0 & & \\
\hline-3 & -2 & -1 & 0 & 1 & 2 & 3 & & \\
\hline $\begin{array}{l}\text { Strongly } \\
\text { Disagree }\end{array}$ & Disagree & $\begin{array}{l}\text { Somewhat } \\
\text { Disagree }\end{array}$ & $\begin{array}{l}\text { Neither Agree } \\
\text { Nor Disagree }\end{array}$ & $\begin{array}{l}\text { Somewhat } \\
\text { Agree }\end{array}$ & Agree & $\begin{array}{l}\text { Strongly } \\
\text { Agree }\end{array}$ & & \\
\hline \multicolumn{9}{|c|}{ 4. It wouldn't take much to make me abandon the non-financial goals (scores of 7 ). } \\
\hline 0 & 0 & 0 & 0 & 0 & 0 & 0 & & \\
\hline-3 & -2 & -1 & 0 & 1 & 2 & 3 & & \\
\hline Strongly & Disagree & Somewhat & Neither Agree & Somewhat & Agree & Strongly & & \\
\hline Disagree & & Disagree & Nor Disagree & Agree & & Agree & & \\
\hline \multicolumn{9}{|c|}{ 5. I think the non-financial goals (scores of 7 ) are good targets to strive for. } \\
\hline & & $\circ$ & $\circ$ & $\circ$ & $\circ$ & $\circ$ & & \\
\hline-3 & -2 & -1 & 0 & 1 & 2 & 3 & & \\
\hline Strongly & Disagree & Somewhat & Neither Agree & Somewhat & Agree & Strongly & & $\checkmark$ \\
\hline Hstart I & Intern... $\%$ & Micro... * & & & & & (9) $12: 35 \mathrm{~F}$ & PM \\
\hline
\end{tabular}




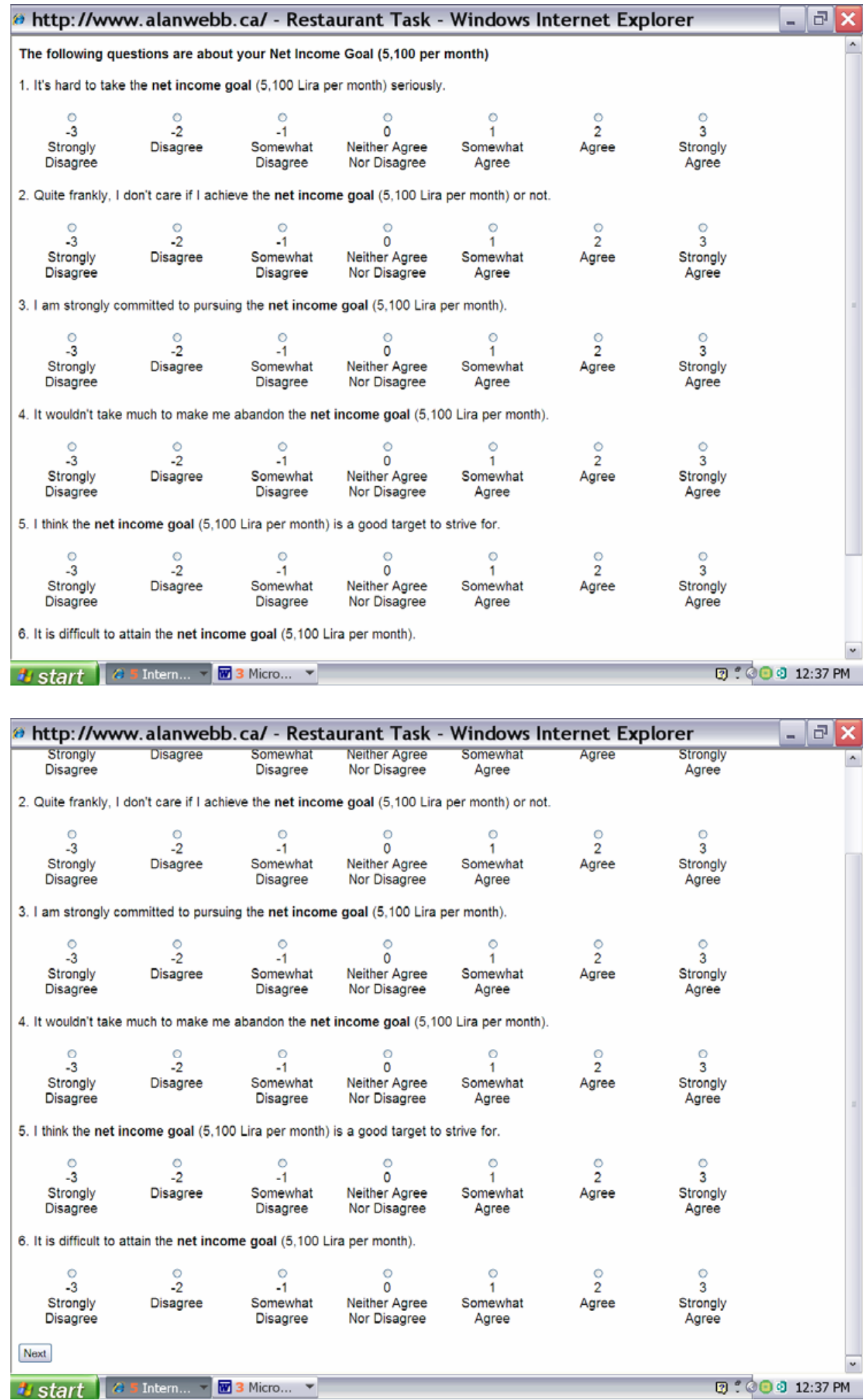




\begin{tabular}{|c|c|c|c|c|c|c|c|c|}
\hline http://w & alanwe & $\mathrm{ca} /$ - Res & Jrant Task & Nindows & rnet $\mathrm{E}$ & rer & & $\nabla x$ \\
\hline Final Questi & & & & & & & & \\
\hline 1. How difficult c & $u$ find the ta & investing in $\mathrm{c}$ & mer service, for & uality, restau & mosphere & enu variety? & & \\
\hline $\begin{array}{c}0 \\
-3\end{array}$ & $\begin{array}{l}0 \\
-2\end{array}$ & $\begin{array}{c}0 \\
-1\end{array}$ & $\begin{array}{l}0 \\
0\end{array}$ & $\begin{array}{l}0 \\
1\end{array}$ & 2 & $\begin{array}{l}0 \\
3\end{array}$ & & \\
\hline${ }^{-3}$ & Difficult & Somewhat & Neither Easy & Somewhat & $\stackrel{2}{2}$ & $\begin{array}{c}3 \\
\text { very }\end{array}$ & & \\
\hline Difficult & & Difficult & Nor Difficult & Easy & & Easy & & \\
\hline 2. How difficult c & $u$ find the or & task of maxin & $g$ your bonus pa & & & & & \\
\hline $\begin{array}{c}0 \\
-3\end{array}$ & $\begin{array}{c}0 \\
-2\end{array}$ & $\begin{array}{l}0 \\
-1\end{array}$ & $\circ$ & $\circ$ & 울 & 욱 & & \\
\hline Very & Difficult & Somewhat & Neither Easy & Somewhat & Easy & Very & & \\
\hline Difficult & & Difficult & Nor Difficult & Easy & & Easy & & \\
\hline 3. Many times, I & to check so & $\mathrm{g}$ before $1 \mathrm{~m}$ & о & 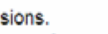 & & & & \\
\hline 0 & 0 & 0 & $\circ$ & $\circ$ & $\circ$ & $\circ$ & & \\
\hline-3 & -2 & -1 & 0 & 1 & 2 & 3 & & \\
\hline $\begin{array}{l}\text { Strongly } \\
\text { Disagree }\end{array}$ & Disagree & $\begin{array}{l}\text { Somewhat } \\
\text { Disagree }\end{array}$ & $\begin{array}{l}\text { Neither Agree } \\
\text { Nor Disagree }\end{array}$ & $\begin{array}{c}\text { Somewhat } \\
\text { Agree }\end{array}$ & Agree & $\begin{array}{l}\text { Strongly } \\
\text { Agree }\end{array}$ & & \\
\hline 4. I had to think & a lot of diff & things at the & ne time to succe & ully perform t & & & & \\
\hline $\begin{array}{c}0 \\
-3\end{array}$ & $\begin{array}{c}0 \\
-2\end{array}$ & $\begin{array}{c}0 \\
0 \\
-1\end{array}$ & $\begin{array}{l}0 \\
0\end{array}$ & 1 & $\begin{array}{l}\circ \\
2\end{array}$ & $\begin{array}{l}0 \\
3\end{array}$ & & \\
\hline $\begin{array}{l}\text { Strongly } \\
\text { Disagree }\end{array}$ & Disagree & $\begin{array}{l}\text { Somewhat } \\
\text { Disagree }\end{array}$ & $\begin{array}{l}\text { Neither Agree } \\
\text { Nor Disagree }\end{array}$ & $\begin{array}{l}\text { Somewhat } \\
\text { Agree }\end{array}$ & Agree & $\begin{array}{l}\text { Strongly } \\
\text { Agree }\end{array}$ & & \\
\hline 5. It was difficult & ding how $\mathrm{m}$ & spend on ea & ctivity in order & chieve the ne & e goal & & & \\
\hline & $\circ$ & 0 & 잉 & 0 & $\circ$ & 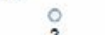 & & \\
\hline${ }^{-3}$ & -2 & -1 & 0 & 1 & 2 & 3 & & \\
\hline $\begin{array}{l}\text { Strongly } \\
\text { Disagree }\end{array}$ & Disagree & $\begin{array}{l}\text { Somewhat } \\
\text { Disagree }\end{array}$ & $\begin{array}{l}\text { Neither Agree } \\
\text { Nor Disagree }\end{array}$ & $\begin{array}{l}\text { Somewhat } \\
\text { Agree }\end{array}$ & Agree & $\begin{array}{l}\text { Strongly } \\
\text { Agree }\end{array}$ & & \\
\hline 6. It was difficult & etermine the & act of the resc & e allocation dec & is on net ince & & & & \\
\hline $\begin{array}{c}0 \\
-3 \\
-3\end{array}$ & $\begin{array}{c}0 \\
-2\end{array}$ & $\stackrel{\circ}{-1}$ & 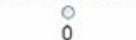 & $\stackrel{\circ}{1}$ & 운 & $\begin{array}{l}0 \\
3\end{array}$ & & \\
\hline $\begin{array}{l}\text { Strongly } \\
\text { Disaree }\end{array}$ & Disagree & Somewhat & $\begin{array}{l}\text { Neither Agree } \\
\text { Nor Disagaree }\end{array}$ & $\begin{array}{l}\text { Somewhat } \\
\text { Arree }\end{array}$ & Agree & $\begin{array}{l}\text { Strongly } \\
\text { Aaree }\end{array}$ & & \\
\hline
\end{tabular}

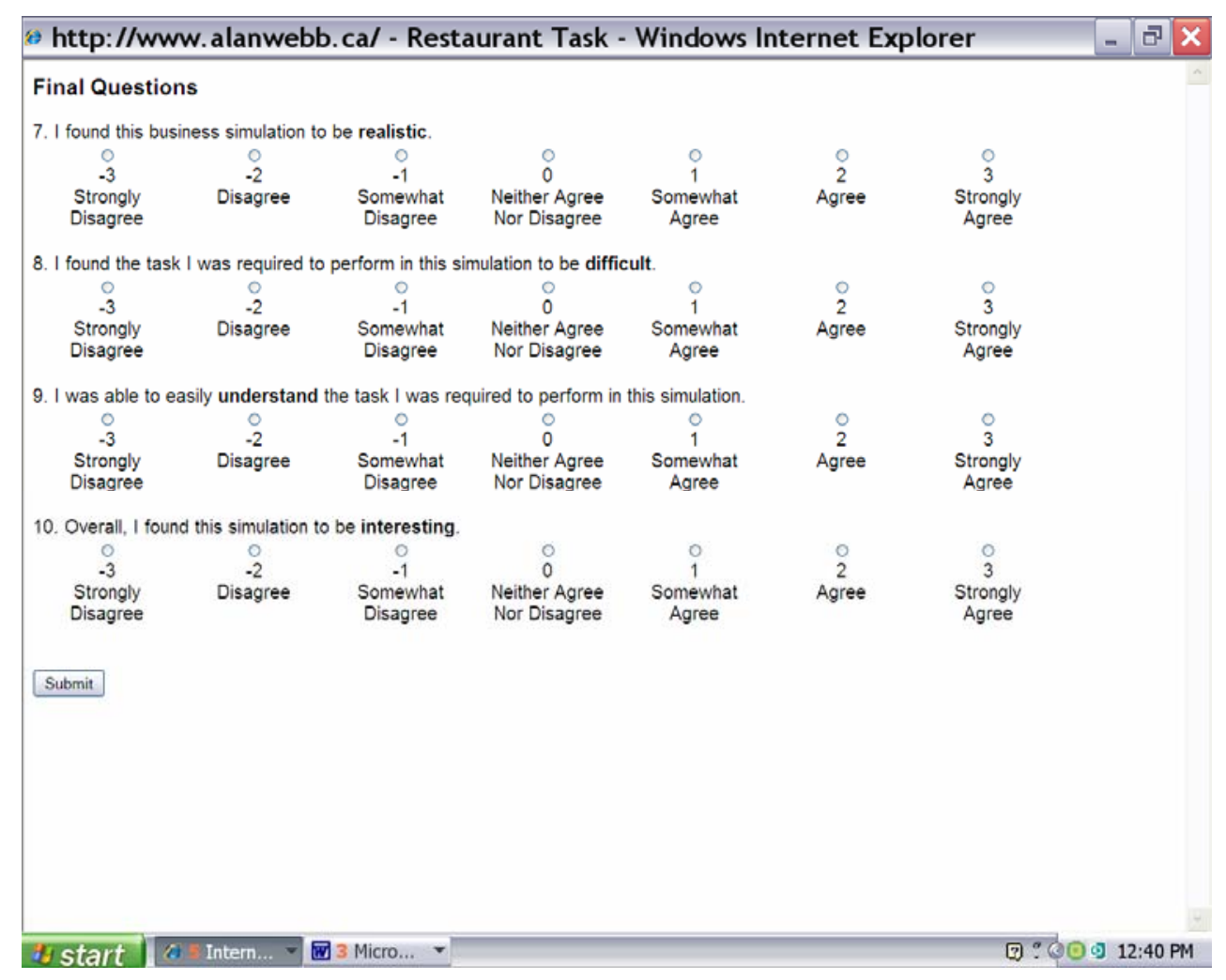




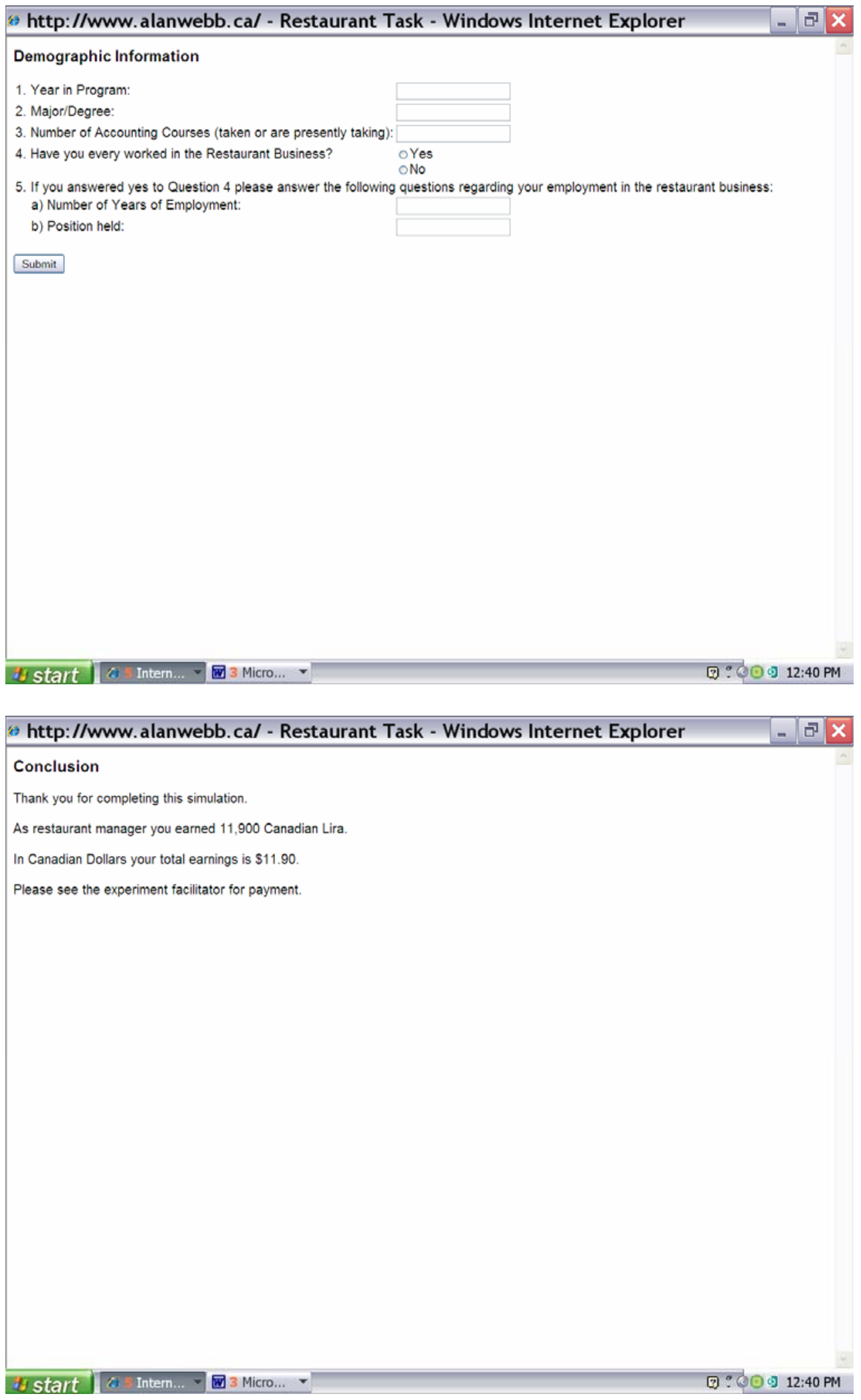

